University of Louisville

ThinkIR: The University of Louisville's Institutional Repository

Electronic Theses and Dissertations

$12-2019$

\title{
GC×GC-MS and parallel 2DLC-MS based untargeted metabolomics.
}

Md Aminul Islam Prodhan

University of Louisville

Follow this and additional works at: https://ir.library.louisville.edu/etd

Part of the Analytical Chemistry Commons

\section{Recommended Citation}

Prodhan, Md Aminul Islam, "GC×GC-MS and parallel 2DLC-MS based untargeted metabolomics." (2019). Electronic Theses and Dissertations. Paper 3307.

https://doi.org/10.18297/etd/3307

This Doctoral Dissertation is brought to you for free and open access by ThinkIR: The University of Louisville's Institutional Repository. It has been accepted for inclusion in Electronic Theses and Dissertations by an authorized administrator of ThinkIR: The University of Louisville's Institutional Repository. This title appears here courtesy of the author, who has retained all other copyrights. For more information, please contact thinkir@louisville.edu. 
GC $\times$ GC-MS AND PARALLEL 2DLC-MS BASED UNTARGETED METABOLOMICS

By

Md Aminul Islam Prodhan

\begin{abstract}
A Dissertation
Submitted to the Faculty of the

College of Arts and Sciences of the University of Louisville

in Partial Fulfillment of the Requirements

for the Degree of
\end{abstract}

Doctor of Philosophy in Chemistry

Department of Chemistry

University of Louisville

Louisville, Kentucky 40208

December, 2019 

GC×GC-MS AND PARALLEL 2DLC-MS BASED UNTARGETED METABOLOMICS

By

Md Aminul Islam Prodhan

A Dissertation Approved on

December 2, 2019

by the following Dissertation Committee:

Dissertation Chair

Dr. Xiang Zhang

Dr. Francis P Zamborini

Dr. Richard P Baldwin

Dr. Michael H Nantz

Dr. Shahid P Baba 


\section{DEDICATION}

This dissertation is dedicated to my parents. 


\section{ACKNOWLEDGEMENTS}

Alhamdulillah (All praise goes to Allah- the almighty creator). I would first praise the Almighty Allah-the creator, protector, provider, cherisher, nourisher, and sustainerwho created me and provided me with all the capabilities, sustenance and blessings. I deeply feel the assistance of Allah in every step of my life including my graduate study.

Special thanks goes to my great mentor, Dr. Xiang Zhang. His excellent guidance and encouragement helped me shape my scientific ability. During the graduate study, he showed me how to be an independent researcher. He taught me how to think independently. Most importantly he taught me the research ethics. It was an absolute privilege to work under his supervision. His sincere attention to my development both scientifically and personally left a deep impression on me and helped me reshape myself. I would like to express my deep appreciation to him and wish him happiness and blessings.

I would like to thank the members of Dr. Zhang's laboratory, especially Mrs. Xinmin Yin, for her support and technical assistance. I also would like to thank Ms. Biyun Shi, Dr. Xiaoli Wei, Dr. Imhoi Koo, Dr. Liqing He and Mrs. Fang Yang for their help during my graduate study.

Appreciation and thanks are extended to the members of my supervisory committee for their assistance and valuable advice in the completion of my graduate study: Dr. Francis P Zamborini, Dr. Richard P Baldwin, Dr. Michael H. Nantz, Dr. Shahid P Baba and Dr. 
Hichem Frigui. Special thanks goes to Dr. Sadakatali Gori and Dr. Pawel Lorkiewicz for their suggestions and encouragement. I also acknowledge the staff of Chemistry Department at the University of Louisville for their help in meeting all the requirements for this degree, especially Mr. Steve Riley for his help in instrument troubleshooting.

I am very grateful to all of my past mentors and the professors of my undergraduate study, including Prof. Abdul Quiyyum, Prof. Sarwar Ahmed Chowdhury, Prof. Zahangir Alam, Prof. Mohammad Ismail, Prof. Ajoy Kumar Das, Prof. A. N. M. Hamidul Kabir, Prof. Sayed Md. Shamsuddin, Prof. Md. Nurul Amin, Prof. Mohammad Nurnabi, Prof. Dilruba Haque, Prof. Mohammed Mizanur Rahman, Prof. A. F. M. Mustafizur Rahman, Prof. Papia Haque, Prof. Ashequl Alam Rana, Prof. Ahmed Ismail Mostafa, Prof. Qamaruzzaman, Prof. Mohammad Mainul Karim, Prof. Md. Ashraful Islam Molla, Prof. Shams Tania Afroza Islam, Prof. Md. Ashaduzzaman, Prof. Shah Md. Masum, at the Department of Applied Chemistry and Chemical Engineering, University of Dhaka, Bangladesh.

I would like to thank my friends Mohammad Ali Tareq, Ahmed Abdullah Sleman, Dr. Ahmed Fahmi Khalifa, Ahmed Saffie, Mostafa Izz-al-din from Department of Computer Engineering and Computer Science and Department of Bioengineering at the University of Louisville for their valuable help in MATLAB computing.

Finally, I extend my deepest gratitude to my parents (Md Jalal Uddin Prodhan \& Rahima Khatun), wife (Tahmida Binte Taiyob Ali), kids (Zainab Binte Amin \& Abdullah Bin Amin), younger brother (Abdullah Al-Mamun), younger sister (Sumaiya Tasnin), maternal uncles (Kazi Ebadat Hossain \& Md Kamrul Hasan), friend (Dr. Akkas Ali) and 
all other family members, who provided me with the greatest love and support throughout my life. 


\begin{abstract}
GC×GC-MS AND PARALLEL 2DLC-MS BASED UNTARGETED METABOLOMICS
\end{abstract}

\author{
Md Aminul Islam Prodhan
}

December 2, 2019

Untargeted metabolomics aims to analyze as many metabolites as possible in a biological system. Due to the vast complexity of the metabolome, bioanalytical platforms have been developed for untargeted metabolomics. While comprehensive two-dimensional gas chromatography mass spectrometry (GC×GC-MS) has been used in different scientific fields, its use in untargeted metabolomics is limited due to several challenges, such as complicated data analysis and limited accuracy of metabolite identification. Twodimensional liquid chromatography mass spectrometry (2DLC-MS) has also been employed in untargeted metabolomics, but with limited metabolite coverage.

This dissertation describes the efforts in developing new data analysis algorithms to improve the accuracy of metabolite identification in GC×GC-MS as well as integration of multiple analytical techniques for increased metabolite coverage and high confidence of metabolite quantification and pathway assignment.

This dissertation is divided into six chapters. Chapter One provides the overview of the current methodologies for untargeted metabolomics. Chapters Two, Three and Four describe the methods developed for the calculation of the second dimension retention index $\left({ }^{2} I\right)$ in GCxGC-MS. Specifically, Chapter Two introduces a surface fitting approach to 
${ }^{2} I$ calculation using $n$-alkanes as reference compounds and considering the second dimension separation as in pseudo-isothermal mode. Chapter Three describes an improved method for calculation of ${ }^{2} I$ by considering the second dimension separations in its actuality, i.e., temperature-programmed mode. Chapter Four introduces a universal reference system for ${ }^{2} I$ calculation to improve retention map coverage that allows the use of any compounds as reference compounds while keeping retention scale in conventional $n$-alkane scale. Chapter Five introduces a method that integrates the GC $\times$ GC-MS and parallel 2DLC-MS platforms for untargeted metabolomics to achieve higher metabolite coverage and accurate metabolite quantification. Chapter Six summarizes the overall scientific contribution of this dissertation. 


\section{TABLE OF CONTENTS}

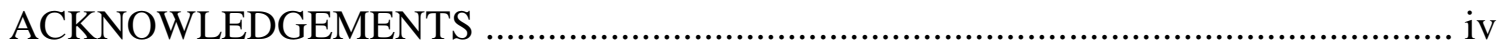

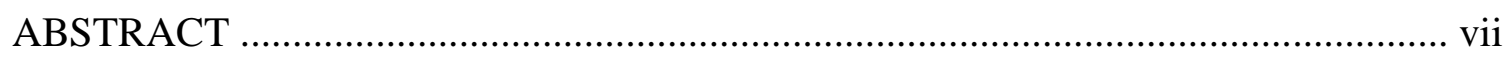

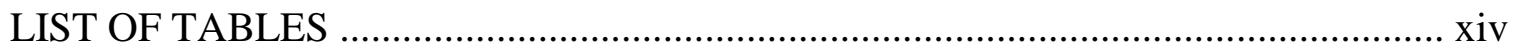

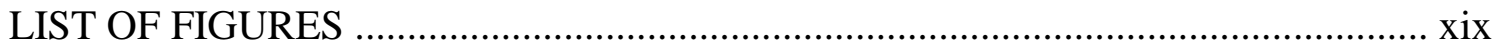

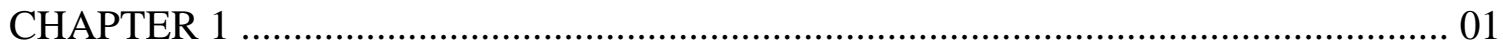

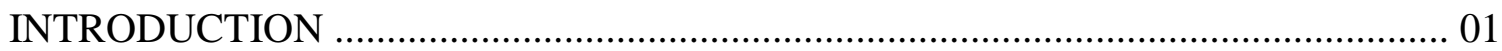

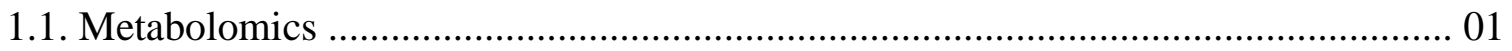

1.2. Technologies Employed in Untargeted Metabolomics ....................................... 02

1.2.1. Gas Chromatography Mass Spectrometry (GC-MS) ................................. 03

1.2.2. Comprehensive Two-dimensional Gas Chromatography Mass Spectrometry

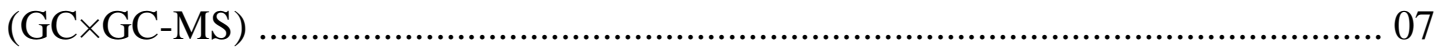

1.2.3. Liquid Chromatography Mass Spectrometry (LC-MS) ............................. 11

1.2.4. Two-dimensional Liquid Chromatography Mass Spectrometry (2DLC-MS)

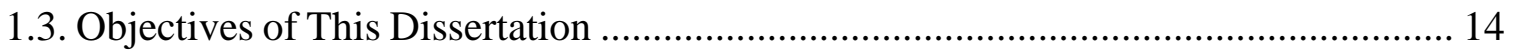


CHAPTER 2

SURFACE FITTING FOR CALCULATING THE SECOND DIMENSION RETENTION INDEX IN COMPREHENSIVE TWO-DIMENSIONAL GAS CHROMATOGRAPHY

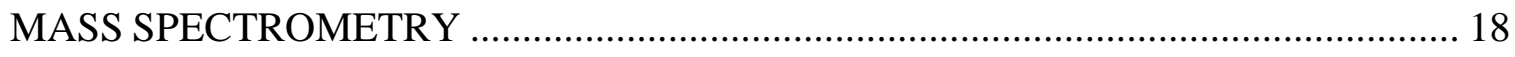

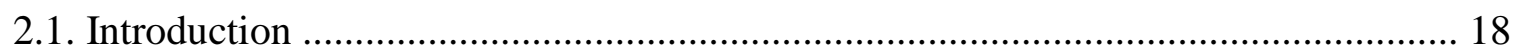

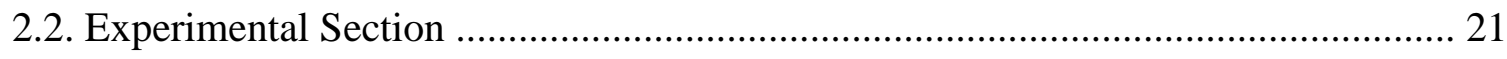

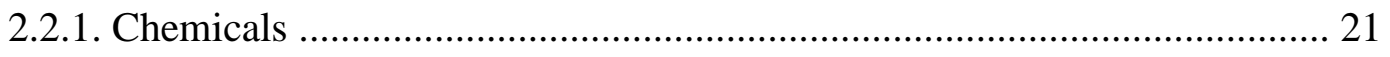

2.2.2. GC×GC-MS Experiment …………………………………………..... 21

2.2.3. Spectrum Deconvolution and Compound Identification ............................. 22

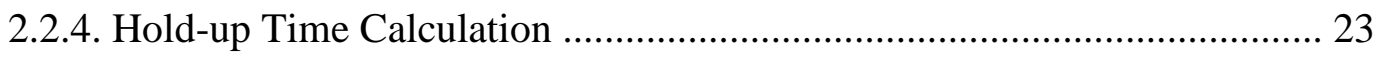

2.2.5. Construction of the Second Dimension Retention Index Surface ............. 24

2.2.6. Evaluation Criteria of Surface Fitting Method ........................................... 25

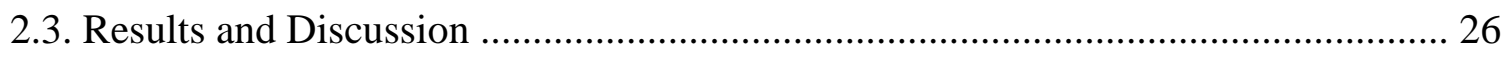

2.3.1. GC $\times$ GC-MS Experimental Variability ................................................. 26

2.3.2. Construction of the Second Dimension Retention Index Surface ............. 28

2.3.3. Calculation of ${ }^{2} I$ of Compounds in MegaMix A \& MegaMix B .......... 34

2.3.4. Robustness of Surface Fitting to Variations of Instrumental Settings ....... 47

2.3.5. Comparison with Literature Reported ${ }^{2} I$ Values ..................................... 53

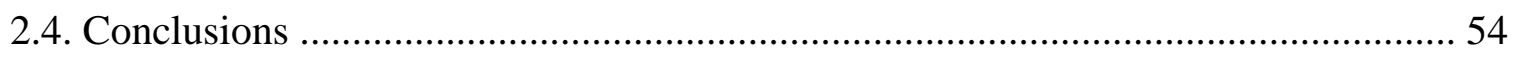

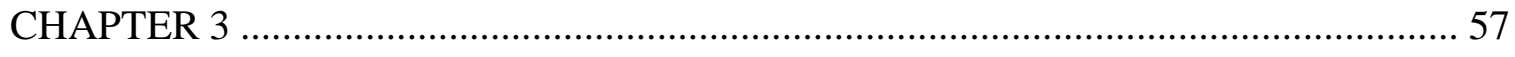

CALCULATING THE RETENTION INDEX OF SECOND DIMENSION SEPARATION IN COMPREHENSIVE TWO-DIMENSIONAL GAS 
CHROMATOGRAPHY MASS SPECTROMETRY WITHOUT INVOLVING HOLD-

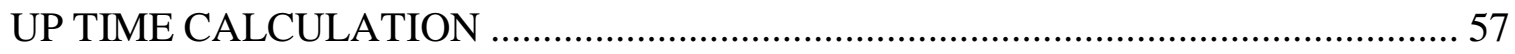

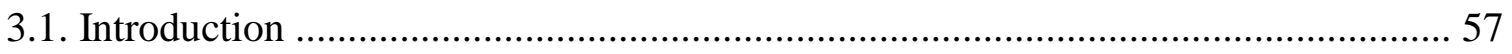

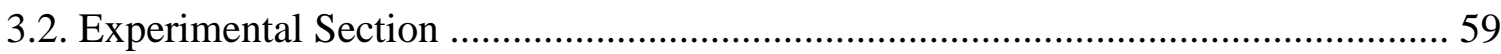

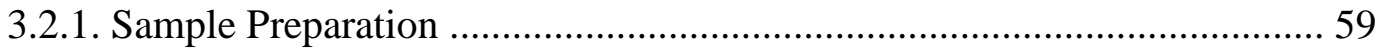

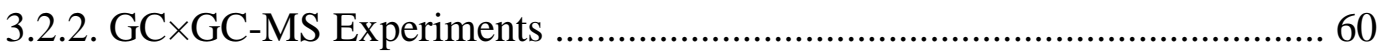

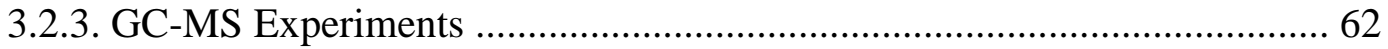

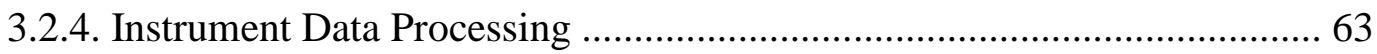

3.2.5. Construction of the Second Dimension Retention Index Surface ............. 64

3.2.6. Selection of Surface Fitting Model ....................................................... 64

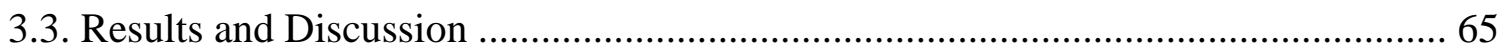

3.3.1. Construction of the Second Dimension Retention Index Surface ............. 66

3.3.2. Calculation and Validation of the ${ }^{2} I$ of Known Compounds .................... 70

3.3.3. Robustness of Surface Fitting to Variations of Instrumental Settings ....... 96

3.3.4. Comparison with ${ }^{2} I$ Calculated by Pseudo-isothermal Condition ......... 102

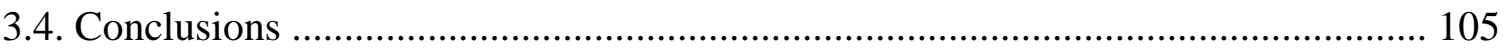

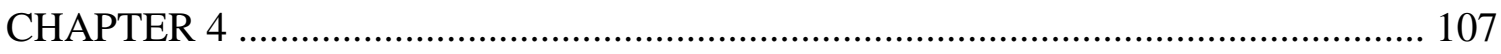

UNIVERSAL REFERENCE SYSTEM FOR CALCULATING THE SECOND DIMENSION RETENTION INDEX IN COMPREHENSIVE TWO-DIMENSIONAL GAS CHROMATOGRAPHY MASS SPECTROMETRY ........................................ 107

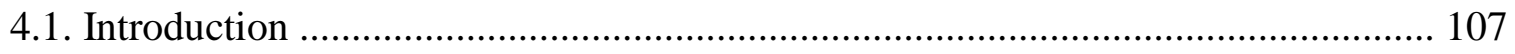

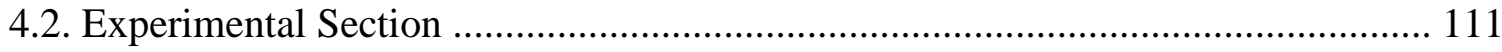




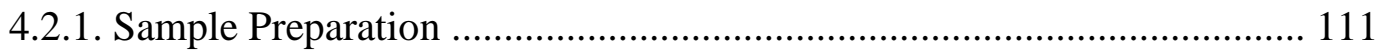

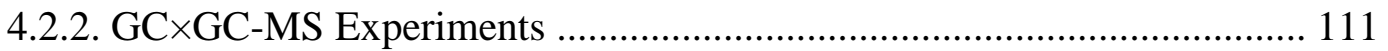

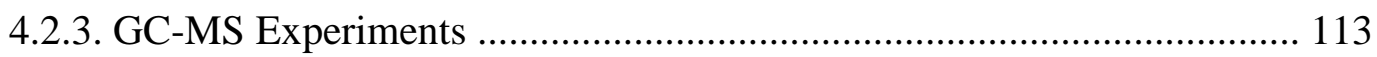

4.2.4. Data Preprocessing ................................................................................. 113

4.2.5. Construction of the Second Dimension Retention Index Surface ........... 114

4.2.6. Selection of Optimal Surface Fitting Model ........................................... 115

4.2.7. Criteria for Evaluating the Surface Fitting ……..................................... 115

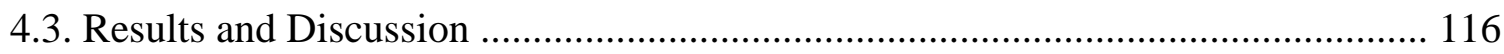

4.3.1. Construction of the Second Dimension Retention Index Surface ........... 116

4.3.2. The Second Dimension Retention Indices of $n$-Alkanes .......................... 124

4.3.3. Calculating the ${ }^{2} I$ of Compound Standards .......................................... 128

4.3.4. Reproducibility and Robustness of Surface Fitting ................................ 141

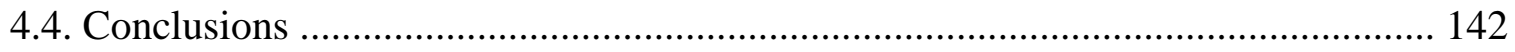

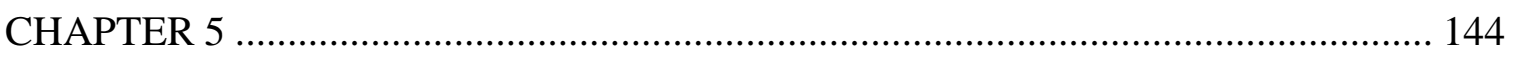

INTEGRATING COMPREHENSIVE TWO-DIMENSIONAL GAS

CHROMATOGRAPHY MASS SPECTROMETRY AND PARALLEL TWODIMENSIONAL LIQUID CHROMATOGRAPHY MASS SPECTROMETRY FOR

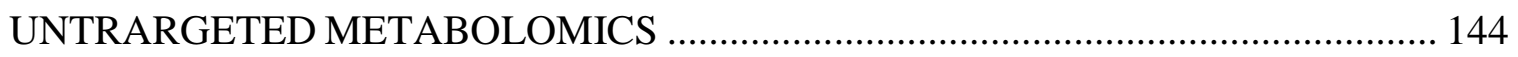

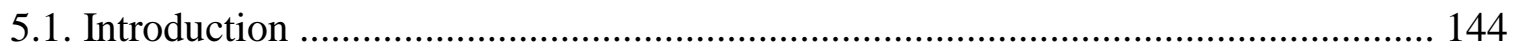

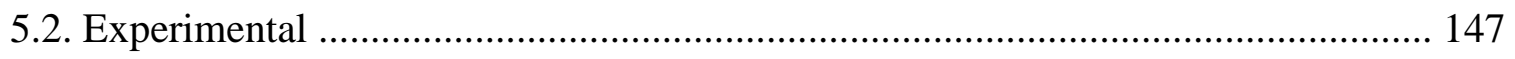

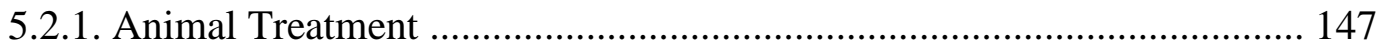

5.2.2. Metabolite Sample Preparation ………..................................................... 147

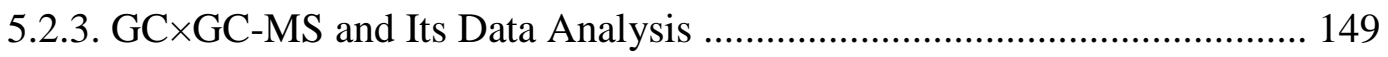




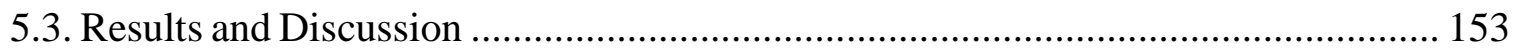

5.3.1. Metabolite Coverage of GC×GC-MS ………………………………..... 153

5.3.2. Metabolite Coverage of Parallel 2DLC-MS ............................................ 157

5.3.3. Integrating GC $\times$ GC-MS and Parallel 2DLC-MS Data for High Metabolite

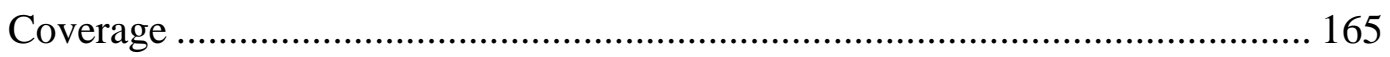

5.3.4. Integrating GC $\times \mathrm{GC}-\mathrm{MS}$ and Parallel 2DLC-MS Data for Accurate

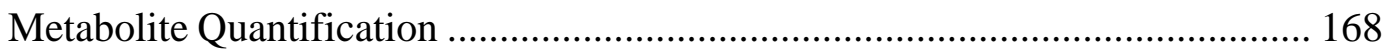

5.3.5. Biomarker Discovery and Pathway Analysis .......................................... 176

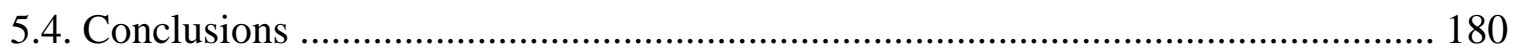

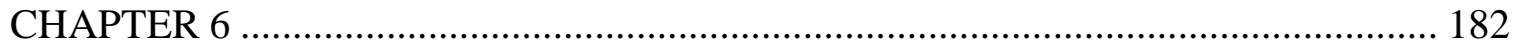

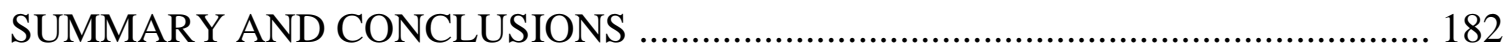

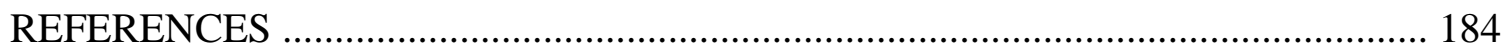

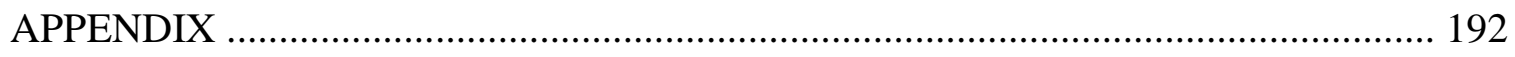

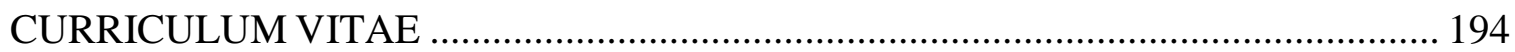




\section{LIST OF TABLES}

Table 2.1. Selection of polynomial surface model 'polyij' for construction of the second dimension retention index surface using GC $\times$ GC-MS data of $n$-alkanes (weight ' $y$ ' and

robust 'LAR') 31

Table 2.2. Results of a ten-fold cross validation of surface fitting using the second dimension retention indices of $n$-alkanes 33

Table 2.3. Results of a ten-fold cross validation of surface fitting using the second dimension retention indices of $n$-alkanes (repeated trial) 34

Table 2.4. Second dimension retention indices of compounds identified from MegaMix A

Table 2.5. Second dimension retention indices of compounds identified from MegaMix B

Table 2.6. Second dimension elution temperature $\left({ }^{2} T_{e}\right)$ of compounds in MegaMix A at different initial temperature with a ramp of $5^{\circ} \mathrm{C} / \mathrm{min}$ and the corresponding adjusted second dimension retention time.

Table 2.7. Second dimension elution temperature $\left({ }^{2} T_{e}\right)$ of compounds in MegaMix B at different initial temperature with a ramp of $5{ }^{\circ} \mathrm{C} / \mathrm{min}$ and the corresponding adjusted second dimension retention time. 
Table 2.8. Second dimension retention indices of compounds identified from MegaMix A

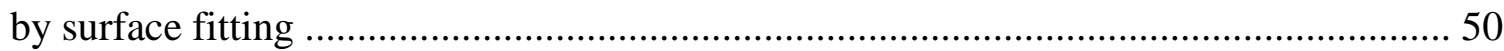

Table 2.9. Second dimension retention indices of compounds identified from MegaMix B

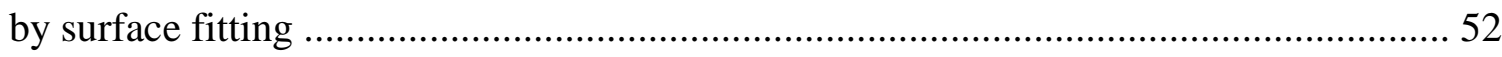

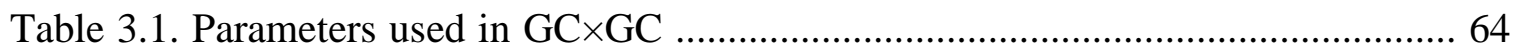

Table 3.2. Data processing parameters used in LECO ChromaTOF software ................. 65

Table 3.3. Selection of polynomial surface model 'polyij' to construct the second dimension retention index surface using GC×GC-MS data of $n$-alkanes (robust 'LAR')

Table 3.4. Lists the calculated $I^{T},{ }^{2} I$ and $\left|I^{T}-{ }^{2} I\right|$ of the compound standards detected in six mixtures of compound standards that were not derivatized 78

Table 3.5. Lists the calculated $I^{T},{ }^{2} I$ and $\left|I^{T}-{ }^{2} I\right|$ of the compound standards detected in ten mixtures that were derivatized by MTBSTFA 81

Table 3.6. Lists the calculated $I^{T},{ }^{2} I$ and $\left|I^{T}-{ }^{2} I\right|$ of the compound standards detected in ten mixtures that were derivatized by MSTFA 86

Table 3.7. Second dimension elution temperature of 80 standard compounds with different ramp rates 94

Table 3.8. Second dimension retention indices of compounds identified from MegaMix A

Table 3.9. Second dimension retention indices of compounds identified from MegaMix B 101 
Table 4.1. Selection of polynomial surface model 'polyij' to construct the second dimension retention index surface using $\mathrm{GC} \times \mathrm{GC}-\mathrm{MS}$ data of $\mathrm{C}_{4}-\mathrm{C}_{25}$ FAMEs 120

Table 4.2. Results of one randomly selected 10 -fold cross validation of surface fitting 'poly35' (case one)

Table 4.3. Results of one randomly selected 10 -fold cross validation of surface fitting 'poly35' (case two)

Table 4.4. Results of one randomly selected 10-fold cross validation of surface fitting 'poly35' (case three)

Table 4.5. Results of one randomly selected 10-fold cross validation of surface fitting 'poly35' (case four)

Table 4.6. Results of one randomly selected 10-fold cross validation of surface fitting 'poly35' (case five) 123

Table 4.7. Second dimension retention indices of 80 compound standards 131

Table 4.8. Second dimension retention indices of compounds identified rom MegaMix A

Table 4.9. Second dimension retention indices of compounds identified from MegaMix B

Table 4.10. Second dimension elution temperature of 80 standard compounds with different ramp rates 141 
Table 5.1. Numbers of detected peaks from pooled samples and biological samples by GC×GCMS and the results of metabolite identification using different matching methods

Table 5.2. Data processing parameters used in LECO ChromaTOF software 158

Table 5.3 Numbers of detected peaks from pooled samples and biological samples by parallel 2DLC-MS and the results of metabolite identification using different matching methods 161

Table 5.4. Details of metabolites identified by MS/MS matching in parallel 2DLC-MS (-) mode. 162

Table 5.5. Details of metabolites identified by MS/MS matching in parallel 2DLC-MS

(+) mode 165

Table 5.6. Metabolites in rat liver detected with significant changes in their abundance levels between $\mathrm{G} 1$ and $\mathrm{G} 2$ by different platforms 173

Table 5.7. $p$-Values of metabolites that were detected by more than one platform with significant changes in their abundance levels between groups 176

Table 5.8. Fold changes of metabolites that were detected by more than one platform with significant changes in their abundance levels between groups 176

Table 5.9. Pathways affected by the treatment difference between groups 180 
Table 5.10. Detection of metabolites from the "biosynthesis of unsaturated fatty acid

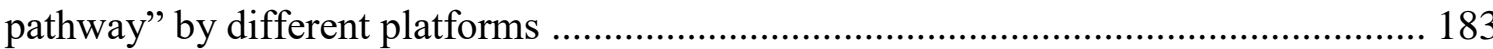




\section{LIST OF FIGURES}

Figure 1.1. Schematic of a GC-MS.

Figure 1.2. Elution order of two hypothetical consecutive reference compounds bracketing a compound of interest. 06

Figure 1.3. An image of a GC $\times$ GC-MS workstation. (Left) The prominent parts of GC $\times$ GC-MS includes the TOF-MS, an autosampler and a GC oven. (Right) Two GC ovens and a column. 08

Figure 1.4. Contour plot of $\mathrm{C}_{7}-\mathrm{C}_{30} n$-alkanes analyzed by $\mathrm{GC} \times \mathrm{GC}-\mathrm{MS}$. 10

Figure 1.5. Isovolatility curve constructed from $\mathrm{C}_{8}-\mathrm{C}_{28} n$-alkanes reference compounds that have even numbers of carbon atoms.

Figure 1.6. Schematic of a LC-MS. 13

Figure 1.7. A sample 2DLC-MS instrumentation. (A) A Q-Exactive Mass Spectrometer with an electrospray source chamber. (B) A Thermo UltiMate 3000 Ultra Performance Liquid Chromatograph with an UV detector, an auto sampler and a temperature controlled compartment containing the column. (C) Two pumps that are used for two different columns. 15 
Figure 2.1. Calculation of the second dimension hold-up time.

Figure 2.2. Cumulative probability curves of standard deviation of the second dimension retention times of $n$-alkanes. 29

Figure 2.3. Distribution of one validation dataset used in 10-fold cross validation.

Figure 2.4. The second dimension retention index surface constructed using the $\mathrm{GC} \times \mathrm{GC}-$ MS data of $n$-alkanes. 36

Figure 2.5. GC $\times \mathrm{GC}-\mathrm{MS}$ separation of 1, 4-dichlorobenzene (black arrow) and other compounds in MegaMix A analyzed in temperature-programmed mode at different column temperatures. 42

Figure 2.6. Correlation of the second dimension retention index of 44 compounds calculated by conventional isovolatility curves and by surface fitting approach.

Figure 3.1. Two-dimensional separation space of $n$-alkanes analyzed in temperatureprogrammed mode using different ramp rates.

Figure 3.2. The second dimension retention index surface constructed using the GCxGCMS data of $n$-alkanes. 72

Figure 3.3. Validation of the second dimension retention indices ${ }^{2} I$ calculated using surface fitting method and its corresponding $I^{T}$ calculated from GC-MS data. (A) 
Correlation between ${ }^{2} I$ of 322 compound standards with their corresponding $I^{T}$. (B)

Deviation between $I^{T}$ and ${ }^{2} I$, i.e., $\left|I^{T}-{ }^{2} I\right|$.

Figure 3.4. Effect of derivatization agent on $\left|I^{T}-{ }^{2} I\right|$. (A) Distribution of $\left|I^{T}-{ }^{2} I\right|$ of MTBSTFA derivatized compounds vis. $\left|I^{T}-{ }^{2} I\right|$ of corresponding compounds derivatized by MSTFA.

Figure 3.5. Effect of elution temperature on retention index calculation. (A) Some randomly selected compounds from MegaMix A and MegaMix B at different ramp rate. (B) Average $\left|I^{T}-{ }^{2} I\right|$ for MegaMix $\mathrm{A}$ and MegaMix $\mathrm{B}$ at different ramp rates.

Figure 3.6. Effect of hold-up time calculation on the deviation $\left|I^{T}-{ }^{2} I\right| .{ }^{2} I_{a}$ is the second dimension retention index calculated by without involving hold-up time calculation. ${ }^{2} I_{b}$ is the second dimension retention index calculated by involving hold-up time calculation. (A) Distribution of $\left|I^{T}-{ }^{2} I\right|$ by approximating the second dimension separation as in pseudo-isothermal mode vis. $\left|I^{T}-{ }^{2} I\right|$ of considering the second dimension separation in its actuality, i.e., temperature-programmed mode. The red line shows the result of linear fitting while the blue and pink lines are fitting confidence with $95 \%$ confidence interval. (B) Box plot of $\left|I^{T}-{ }^{2} I\right|$ calculated using the two methods. 105

Figure 4.1. Second dimension retention map of $\mathrm{C}_{4}-\mathrm{C}_{25}$ FAMEs analyzed in temperatureprogrammed mode using different ramp rates. Inflection points in the curve indicate the second dimension column temperature of $285^{\circ} \mathrm{C}$, from which the corresponding $\mathrm{C}_{4}-\mathrm{C}_{25}$ 
FAMEs were analyzed in isothermal mode for 13 min

Figure 4.2. Second dimension retention index surface constructed using the $\mathrm{GC} \times \mathrm{GC}-\mathrm{MS}$ data of $\mathrm{C}_{4}-\mathrm{C}_{25}$ FAMEs.

Figure 4.3. Second dimension retention map constructed from the $\mathrm{C}_{4}-\mathrm{C}_{25}$ FAMEs and $n$ alkanes data.

Figure 4.4. Standard deviation of calculated ${ }^{2} I$ of each $n$-alkane among the seventeen chromatographic runs with different ramp rates.

Figure 4.5. Retention index variation of $n$-alkanes between the calculated ${ }^{2} I$ from their hypothetical value $100 \times n . n$ is the number of carbon atoms in an $n$-alkane. 128

Figure 4.6. Second dimension retention map of $n$-alkanes.

Figure 4.7. Correlation between the linear retention index of compounds calculated from GC-MS data and their corresponding second dimension linear retention indices calculated using the developed method. The compounds include 80 compound standards and compounds in MegaMix A $\quad$ and $\quad$ MegaMix $\quad$ B.

Figure 4.8. Distribution of standard deviation of the calculated ${ }^{2} I$ of the 80 standard compounds among different ramp rates.

Figure 5.1. Two compounds co-eluted from the first dimension GC but separated by the second dimension GC. (A) is a three-dimensional view, and (B) is a contour plot. 
Figure 5.2. Overlap of metabolite identification.

Figure 5.3. PLS-DA analysis of the metabolite profiles of all samples. (A) is the clustering result of parallel 2DLC-MS (-) data. (B) is the clustering result of parallel 2DLC-MS (+) data. (C) is the cross validation result of the PLS-DA for analysis of parallel 2DLC-MS () data. The magnitudes of $\mathrm{R}^{2}$ and $\mathrm{Q}^{2}$ are $\left(\mathrm{R}^{2}=0.91 \mathrm{Q}^{2}=0.40\right)$. (D) is the cross validation results of the PLS-DA for analysis of parallel 2DLC-MS (+) data with $\mathrm{R}^{2}=0.72$ and $\mathrm{Q}^{2}=$ 0.12. 171

Figure 5.4. PLS-DA analysis of the metabolite profiles of all samples. (A) is the clustering result of GC $\times$ GC-MS data. (B) is the cross validation results of the PLS-DA for analysis of $\mathrm{GC} \times \mathrm{GC}-\mathrm{MS}(+)$ data with $\mathrm{R}^{2}=1.0$ and $\mathrm{Q}^{2}=-0.18$

Figure 5.5. Overlap of metabolites detected with significant changes in their abundance levels between groups by three platforms. 173

Figure 5.6. The regulation directions of metabolites that showed significant changes in their abundance levels between groups in three platforms, GC×GC-MS, parallel 2DLC-MS (-) and parallel 2DLC-MS (+). 176

Figure 5.7. Samples of instrument response of a metabolite affecting the quantification accuracy of that metabolite. (A) Three-dimensional chromatographic peak of 1-ornithine detected by GC $\times$ GC-MS. (B) Extracted ion chromatograms of 1-ornithine in a randomly selected biological sample detected by parallel 2DLC-MS (-). (C) Extracted ion chromatograms of 1-ornithine in a randomly selected biological sample detected by parallel 2DLC-MS (+). (D) Three-dimensional chromatographic peak of taurine detected by GC $\times$ GC-MS. (E) Extracted ion chromatograms of taurine in a randomly selected biological 
sample detected by parallel 2DLC-MS (-). (F) Extracted ion chromatograms of taurine in a randomly selected biological sample detected by parallel 2DLC-MS (+).

Figure 5.8. Overlap of the pathways that were affected using different sets of significant metabolites for metabolic pathway analysis. 


\section{CHAPTER 1}

\section{INTRODUCTION}

\subsection{Metabolomics}

Metabolome is the collection of all small compounds in an organism (i.e., metabolites, with molecular weight typically under 1500 Dalton. Metabolites are the intermediates and products of all biological processes that take place in a biological system. Therefore, a metabolome is the result of a large network of metabolic reactions. The output from one enzymatic chemical reaction are input of other biochemical reactions. Comparing with genome and proteome, the metabolome is much more dynamic and complex because each metabolite is biochemically, spatially and temporally defined. ${ }^{1,2}$ Metabolomics is the systematic study of the metabolite formation. Inherent complexity of the metabolome in a biological sample makes the metabolomics a multidisciplinary research area that requires knowledge in Chemistry, Biology, Computer Science, Biostatistics, Biomedical Science, etc. Broadly, metabolomics methodology falls into two major categories, untargeted metabolomics and targeted metabolomics. 
Untargeted metabolomics compares the difference of abundance levels of metabolites detected in two or more groups ( e.g., control group and test group), to enlighten the abundance difference of metabolites that may provide the insight about relevance to the specific biological conditions. The goal of untargeted metabolomics is to detect as many metabolites as possible, and it allows the collection of data without prior knowledge about metabolites. Owing to the large number of metabolites, diverse chemical characteristics and huge concentration differences between metabolites, multiple analysis steps are involved in untargeted metabolomics at every step (i.e., sample preparation, separation methods and instrumental analysis, affects the accuracy of metabolite analysis).

Targeted metabolomics is a quantitative method that focused on a group of chemically characterized and biochemically annotated metabolites. It generally uses internal standards to make the analysis quantitative or semi-quantitative. As predefined lists of metabolites are studied, novel associations between metabolites may be illuminated in the context of specific physiological states. Targeted metabolomics exploits different mass spectrometric methods, (e.g., selected reaction monitoring (SRM) by tandem mass spectrometry, multi-reaction monitoring (MRM), parallel reaction monitoring (PRM), etc.).

\subsection{Technologies Employed in Untargeted Metabolomics}

Metabolites have diverse physical and chemical characteristics, as well as very large concentration difference among them in metabolome. For instance, metabolites can be polar or non-polar, as well as organic or inorganic compounds. ${ }^{3,45}$ These differences in chemical diversity have profound implications in metabolomics. As the chemical diversity of the metabolome is so broad, the analytical technologies needed to isolate and identify 
thousands of chemically distinct metabolites are inherently complex. Furthermore, one does not know the number of metabolites in a biological sample such as human body fluids. It is estimated that at least thousands of metabolites should be detectable. The diverse characteristics and huge number of entities make metabolite separation challenging, in that there is not a singular instrument capable of analyzing all types of metabolites. ${ }^{6-10}$ To date, multiple analytical platforms have been developed and used in untargeted metabolomics. Among those analytical platforms, biological samples are frequently analyzed by gas chromatography mass spectrometry (GC-MS) and/or liquid chromatography mass spectrometry (LC-MS). ${ }^{11-13}$

\subsubsection{Gas Chromatography Mass Spectrometry}

GC-MS is known to be the gold standard technique for analysis of volatile or semivolatile metabolites. Figure 1.1 is a schematic diagram of a GC-MS instrument. In GC-MS based metabolomics, metabolites are first derivatized by a derivatization reagent to make the metabolite volatile and thermally stable. The derivatized metabolites are then separated on a GC column and the separated metabolites are further subjected to mass spectrometer for measurement. The GC column is usually 10 to $60 \mathrm{~m}$

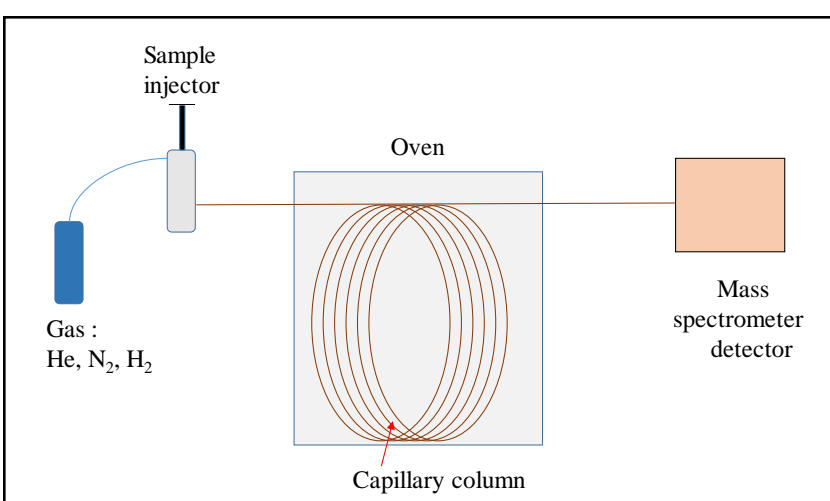

Figure 1.1. Schematic of a GC-MS. long and the stationary phases can be polar, semi non-polar or non-polar. GC separates the metabolites by partitioning the metabolite between the stationary phase and the mobile phase inside the GC column. Specifically, inside of a GC column, a flow of mobile phase, 
usually helium, percolates through an absorbent known as stationary phase. The metabolites initially adsorbed to the surface of the stationary phase followed by evaporation from the stationary phase to the open space of the column. The metabolites are then hit by carrier gas molecules and start travelling down the column. After a short distance, however, they touch the sticky surface of the stationary phase and go through another partitioning process. This process continues until the metabolites enter into mass spectrometer that mostly equipped with an electron ionization (EI) ion source. While GCMS has an excellent resolving capacity to separate metabolites, it has limited peak capacity. Moreover, a GC-MS system requires chemical derivatization of metabolites in order to make them volatile and thermally stable.

Identification of metabolites in GC-MS: GC-MS provides two measures for each metabolite, retention time and an EI mass spectrum. A GC-MS system can detect $600-$ 900 peaks and only about 200 - 300 metabolites can be identified. ${ }^{14}$ Compound identification in analysis of GC-MS data is usually achieved by matching a query spectrum, i.e., an experimental EI mass spectrum, to the mass spectra of compound standards in a reference library. A metabolite in the reference library with the highest spectral similarity measure is usually considered as the metabolite given rise to the query spectrum. This process is entitled mass spectrum matching. ${ }^{15}$ Many mass spectrum matching methods have been developed for analysis of GC-MS data, including composite similarity, ${ }^{16}$ probability-based matching system, ${ }^{17}$ cosine correlation ${ }^{18-21}$ and Hertz similarity index. ${ }^{22}$ Stein and $\mathrm{Scott}^{23}$ examined the performance of composite measure with other measures using National Institute of Standard Technology (NIST) database, while Horai et al. ${ }^{24}$ performed a similar study using MassBank database. Both studies demonstrated that the 
composite measure performed better than other methods. Dr. Xiang Zhang's group ( University of Louisville ) further demonstrated that the identification accuracy of existing methods is about $80 \%,{ }^{25-27}$ likely due to the significant enrichment of reference spectral library and the increased sample complexity. A large spectral library increases the chance that the mass spectrum of the true metabolite is present in the library, but it also increases the chance that highly similar mass spectra from other metabolites are also present. Multiple metabolites in a biological sample may have similar mass spectra, resulting in some metabolites in the mass spectrum library having multiple spectrum matched peaks in the experimental data, and the spectrum with the highest similarity score may not be the true match. In addition, the mass spectrum represents only partial information of the molecular structure of a metabolite. Therefore, the mass spectrum matching based identification method in GC-MS has limited identification accuracy.

Retention index to aid in metabolite identification in GC-MS. Identifying metabolites solely based on mass spectrum matching has inherent limitations. Therefore, additional information such as metabolite separation information (e.g., retention time) can be employed to increase the identification confidence. Since retention time in GC depends on experimental conditions, retention index was introduced to reduce such dependency. Retention index is normalized retention time using homologous reference series mostly $n$ alkanes. Figure 1.2 shows the elution order of two homologous reference compounds ( $x$ and $y$ ) bracketing a compound $(u)$. The retention time of compound $u$ can

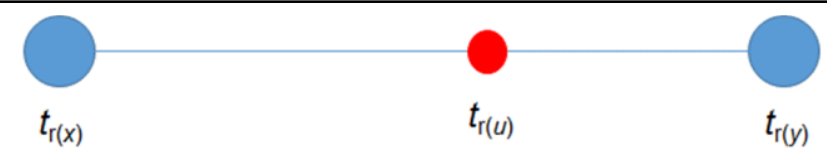

Figure 1.2. Elution order of two hypothetical consecutive reference compounds bracketing a compound of interest. 
be normalized with respect to the retention times of reference compounds $x$ and $y$. This normalized retention time is known as retention index $\left(I_{u}^{T}\right)$.

Two equations have been developed to calculate the retention index based on the operation mode of GC-MS, temperature-programmed mode or isothermal mode. For the temperature-programmed mode, retention time $t_{R}$ of a compound $(u)$ is converted into a linear retention index $I_{u}^{T}$ using the retention times of the two bracketing $n$-alkanes as follows: $:^{28}$

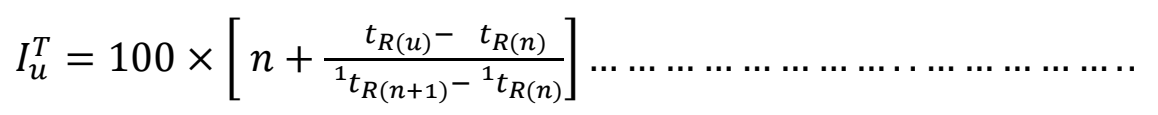

where $n$ is the number of carbon atoms in $n$-alkane, $\quad t_{R(u)}, \quad t_{R(n)}$ and $t_{R(n+1)}$ are the retention times of the compound $u$, the $n$-alkane with $n$ carbon atoms and the $n$-alkane with $n+1$ carbon atoms, respectively.

For isothermal conditions, retention time $t_{R}$ of a compound $(u)$ is converted into Kovats retention index $I$ as follows: ${ }^{29}$

$$
I_{u}=100 \times\left[n+\frac{\log \left({ }^{2} \mathrm{t}_{\mathrm{R}(\mathrm{u})}^{\prime}\right)-\log \left({ }^{2} \mathrm{t}_{\mathrm{R}(\mathrm{n})}^{\prime}\right)}{\log \left({ }^{2} \mathrm{t}_{\mathrm{R}(\mathrm{n}+1)}^{\prime}\right)-\log \left({ }^{2} \mathrm{t}_{\mathrm{R}(\mathrm{n})}^{\prime}\right)}\right] \ldots \ldots \ldots \ldots \ldots \ldots
$$

where $n$ is the number of carbon atoms in $n$-alkane, ${ }^{2} t_{R}^{\prime}$ is the adjusted second-dimension retention time, i.e., the second-dimension retention time of a chromatographic peak minus the retention time of a unretained peak ${ }^{2} t_{M}$.

Both mass spectrum and retention index have been used for compound identification in GC-MS. ${ }^{30-32}$ All of the existing methods employ retention index as a filter to remove the potential false-positive identifications generated by mass spectrum matching. Such an 
analysis strategy uses the retention index and mass spectrum in two separate analysis steps. The sequential nature of the two-step analysis strategy increases the risk of introducing errors from each independent stage since there is no way to correct the errors caused by the previous step. To improve the identification accuracy, Dr. Xiaoli Wei developed a SimMR method that simultaneously evaluates the mass spectrum similarity and the retention index distance using an empirical mixture score function, ${ }^{33}$ and her study demonstrated that the SimMR method outperforms the literature reported compound identification methods by improving the overall identification accuracy up to $1.53 \%$.

\subsubsection{Comprehensive Two-dimensional Gas Chromatography Mass Spectrometry}

Due to the limited peak capacity of GC-MS, ${ }^{34}$ two-dimensional gas chromatography (2DGC) was developed decades ago. Conventional 2DGC technique uses the "heart cutting" method where selected portions of analytes eluted from the first dimension $\left({ }^{1} \mathrm{D}\right)$ column are transferred to the second dimension $\left({ }^{2} \mathrm{D}\right)$ column for further separation to achieve enhanced resolution for the heart-cutting zone. ${ }^{35}$ This technique is unsuitable for untargeted metabolomics owing to its limited peak capacity and longer analysis time. Comprehensive two-dimensional gas chromatography mass spectrometry (GC $\times \mathrm{GC}-\mathrm{MS})$ is the latest development in the 2DGC technique to enhance the GC separation power.

A GC $\times$ GC-MS system has four major components: a first dimension $\left({ }^{1} \mathrm{D}\right)$ column, a modulator, a second dimension $\left({ }^{2} \mathrm{D}\right)$ column and a mass spectrometer. The two columns ${ }^{1} \mathrm{D}$ and ${ }^{2} \mathrm{D}$ are connected in series by a modulator. After the sample is injected into the 
system through an injector, metabolites are initially separated on the ${ }^{1} \mathrm{D}$ column. Metabolites eluted from the ${ }^{1} \mathrm{D}$ column within a certain period of time, termed as modulation period $P_{M}$, are collected as a fraction by the modulator and subjected to the ${ }^{2} \mathrm{D}$ column for further separation. After the separation on the ${ }^{2} \mathrm{D}$ column, metabolites are transferred to the mass spectrometer for measurement. This process is sequential and continuous throughout the analysis until all fractions collected from the ${ }^{1} \mathrm{D}$ column are analyzed by the ${ }^{2} \mathrm{D}$ column and mass spectrometer (Figure 1.3). Therefore, the GC $\times \mathrm{GC}$ MS offers a much increased peak capacity and superior resolution, selectivity, sensitivity, and dynamic range for the analysis of small molecules. ${ }^{36,37}$

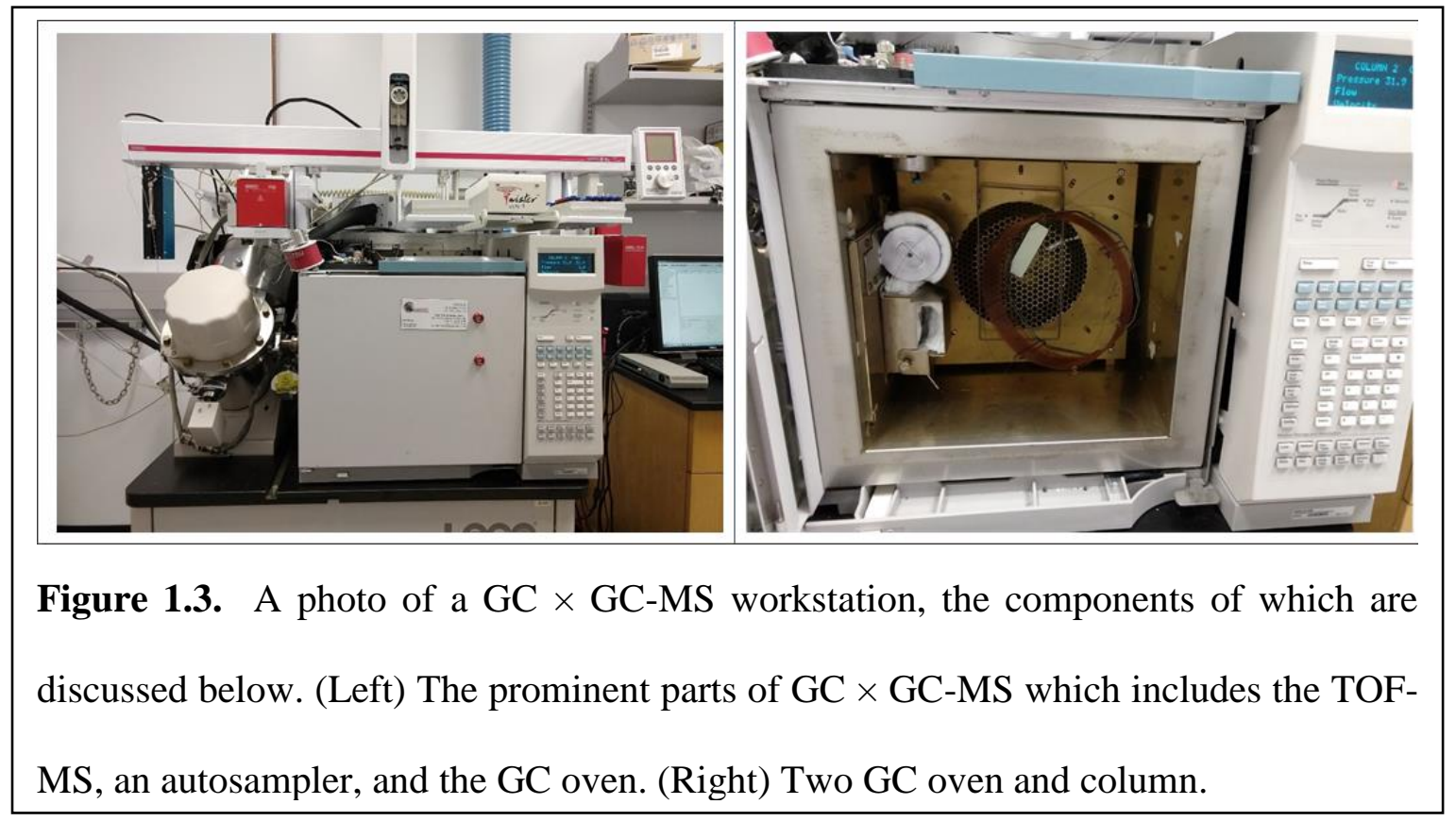

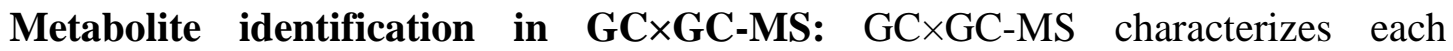
metabolite by its first dimension retention time $\left({ }^{1} t_{R}\right)$, the second dimension retention time $\left({ }^{2} t_{R}\right)$ and an EI mass spectrum. ${ }^{38,39}$ Likewise to GC-MS, identification in GC $\times$ GC-MS also is done by mass spectrum matching as described in section 1.2.1. Identification of 
metabolites solely based on the mass spectrum matching also incurs the similar problem as encountered in GC-MS.

In order to increase the identification accuracy, retention index has also been used in analysis of $\mathrm{GC} \times \mathrm{GC}-\mathrm{MS}$ data. GC $\times \mathrm{GC}-\mathrm{MS}$ has two columns. For the first dimension column, the retention time $\left({ }^{1} t_{R}\right)$ of a compound $u$ is converted into linear retention index $I^{T}$ using Equation (1). While Equation (1) can be used for calculation of the first dimension retention index, it cannot be used for calculation of the second dimension retention index. Several factors hinder the use of Equation (1) for the second dimension retention index calculation. First, the second dimension separation is limited by the modulation time. In order to use Equation (1), the second dimension retention time of a compound of interest must locate between the second dimension retention times of two reference compounds. That is the metabolite of interest and the two reference compounds must have the same first dimension retention times, i.e., eluting from the first dimension column in the same modulation period. However, it is not possible to always get two bracketing reference compounds in the same modulation period. Figure 1.4 shows a sample contour plot of $\mathrm{C}_{7}$ $\mathrm{C}_{30} n$-alkanes analyzed by $\mathrm{GC} \times \mathrm{GC}-\mathrm{MS}$, where $\mathrm{x}$-axis is the first dimension retention time and $y$-axis is the second dimension retention time. The unit of the both axis are in seconds. Specifically, compound $\mathrm{U}_{1}$ eluted between

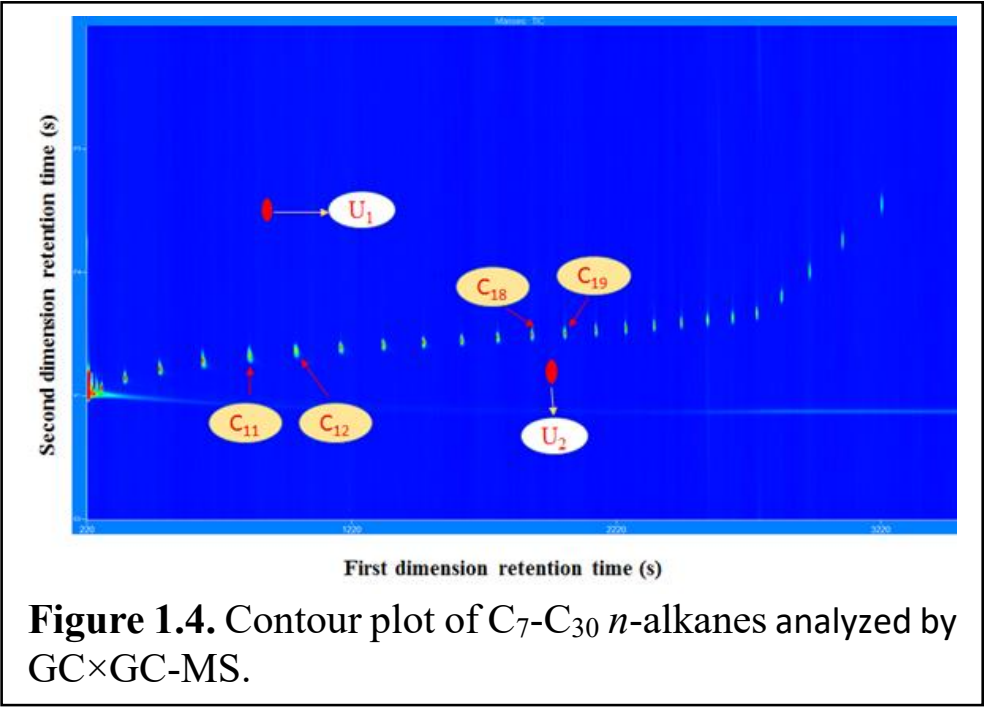


two consecutive $n$-alkanes reference compounds $\mathrm{C}_{11}$ and $\mathrm{C}_{12}$ wherein compound $\mathrm{U}_{2}$ was eluted between reference compounds $\mathrm{C}_{18}$ and $\mathrm{C}_{19}$. Therefore, the first dimension retention index of $\mathrm{U}_{1}$ and $\mathrm{U}_{2}$ can be calculated using the first dimension retention times of their corresponding bracketing reference compounds employing Equation (1). However, there is no bracketing reference compounds eluted after of compounds $U_{1}$ and before $U_{2}$ in yaxis. Therefore, we cannot use Equation (1) to calculate the second dimension retention index of these compounds. In order to solve this problem, a method called "isovolatility curve" has been developed to calculate the second dimension retention index $\left({ }^{2} I\right)$ using Equation (2). ${ }^{40,41}$ The reason for using Equation (2) to calculate ${ }^{2} I$ is that the second dimension separation was considered as pseudo-isothermal as the second dimension column is very short and the resultant temperature change in each modulation period is very small.

The retention time of a compound in the second dimension $\left({ }^{2} t_{R}\right)$ column decreases with increases in elution temperature $\left({ }^{2} T_{e}\right)$. The relation $\left[{ }^{2} t_{R} \sim{ }^{2} T_{e}\right]$ of a compound can be captured by the isovolatility curve. Figure 1.5 shows an example of an isovolatility curve constructed using the $\mathrm{GC} \times \mathrm{GC}$ MS data of $\mathrm{C}_{8}-\mathrm{C}_{28}$ evennumbered $n$-alkanes, where every curve represents one isovolatility curve corresponding to a $n$-alkane compound. Isovolatility curve can be constructed by several different ways while the main

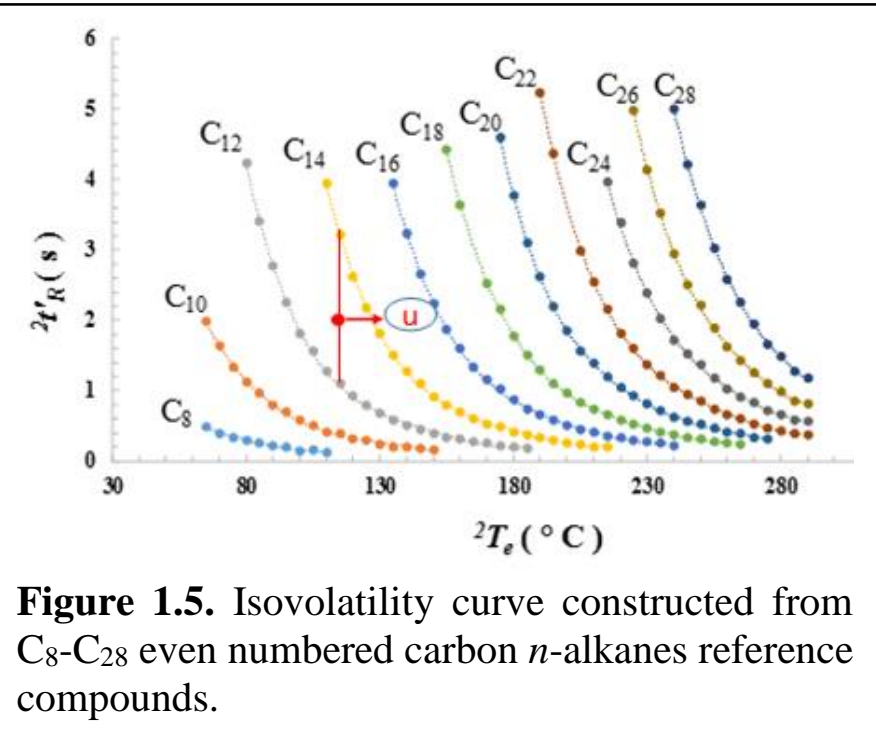


idea is to get the retention time of reference compounds at different elution temperatures. As the second dimension separation considered as pseudo-isothermal, the hold-up time, ${ }^{2} t_{M}$, of reference compounds at different temperatures must be estimated to get the adjusted retention time, ${ }^{2} t_{R}^{\prime}$. The ${ }^{2} t_{R}^{\prime}$ values of the same reference compound at different column temperatures were then used to construct the isovolatility curves of the reference compounds. The isovolatility curves of all reference compounds formed the second dimension retention index map.

After the construction of the isovolatility curves, the ${ }^{2} I$ value of a compound can be calculated using the two adjacent isovolatility curves corresponding to two reference compounds. In Figure 1.5, compound $u$ was eluted between the isovolatility curves of reference compounds $\mathrm{C}_{12}$ and $\mathrm{C}_{14}$. Compound $u$ has its second dimension retention time and the corresponding second dimension elution temperature. If this elution temperature value is interpolated in the isovolatility curve equations of reference compounds $\mathrm{C}_{12}$ and $\mathrm{C}_{14}$, two corresponding retention time values can be obtained at the elution temperature of $u$. Now, ${ }^{2} I$ of compound $u$ can be calculated using Equation (2) as retention times of compound $u$ and the reference compounds are at the isothermal condition (same $\mathrm{x}$-axis value)..$^{40}$

While the first dimension retention index has been used to aid in compound identification ${ }^{42}$, the second dimension retention index ${ }^{2} I$ has not been extensively used in aiding compound identification in GC $\times$ GC-MS due to inaccuracy of ${ }^{2} I$ calculation.

\subsubsection{Liquid Chromatography Mass Spectrometry (LC-MS)}


The liquid chromatography mass spectrometry (LC-MS) has also been used in untargeted metabolomics. ${ }^{43-47}$ In LC-MS, metabolites are separated based on their interaction with mobile phase and stationary phase when the mobile phase moves through the stationary phase in the column under pressure. Figure 1.6 depicts the instrumentation of LC-MS that consists of a pump, an injection valve, a column and a mass spectrometer. A sample is loaded into the column through an injection valve. Pressure created by the pump forces the mobile phase to carry the metabolites to the detector through the LC

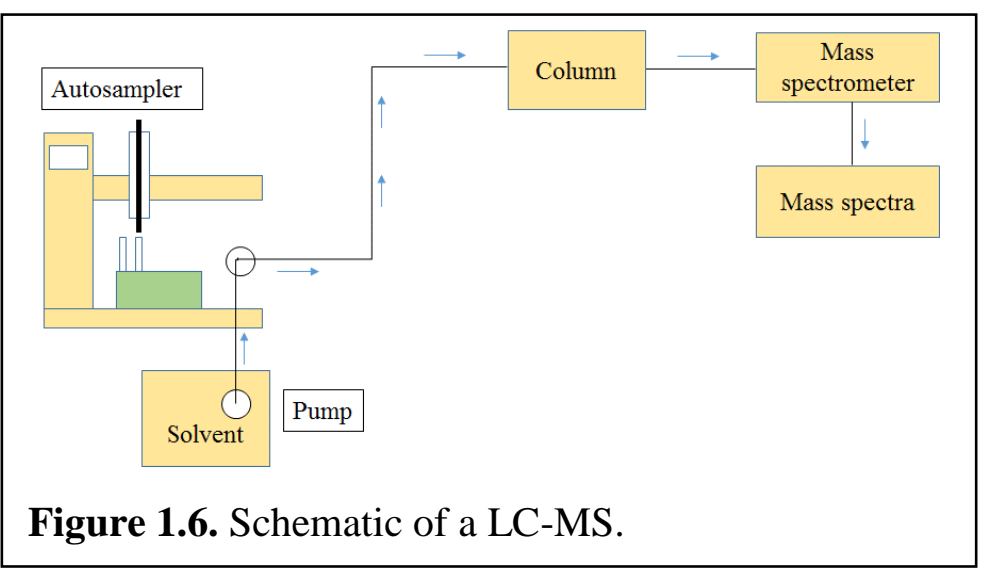
column. The column is packed with stationary phase particles. Depending on the stationary phase particles, the LC can be categorized as normal phase chromatography, reversed-phase chromatography, ionexchange chromatography, etc.

Choices a column stationary phase and the corresponding mobile phase are the most important and challenging decision in LC-MS. Among the different liquid chromatography modes, reversed-phase chromatography (RPC) is the most widely used technique. In RPC, the stationary phase is non-polar (hydrophobic) and the mobile phase is polar. Water mixed with other solvents ( i.e., methanol, isopropanol and/or acetonitrile ) are used as the mobile phase wherein long-chain alkyl groups (i.e., $n$-octadecyl or C18) attached to the surface of spherically shaped silica particles are used as the stationary phase. ${ }^{48}$ Therefore, RPC is suitable for separation of non-polar metabolites. Hydrophilic interaction chromatography 
(HILIC), a form of normal phase chromatography, has also been used to separate metabolites in LC-MS based metabolomics. ${ }^{49}$ In HILIC, mobile phase is water miscible solvents like acetonitrile, methanol, etc. mixed with water and the stationary phase is polar. Therefore, HILIC provides strong retention of metabolites that are unretained under conventional reversed-phase condition.

\subsubsection{Two-dimensional Liquid Chromatography Mass Spectrometry}

Due to the limited peak capacity of one-dimensional LC-MS, two-dimensional liquid chromatography mass spectrometry (2DLC-MS) has been developed to increase the peak capacity of the liquid chromatographic system. Figure 1.7 depicts a sample 2DLCMS system, where different separation mechanisms, such as RPC and HILIC, was incorporated into the 2DLC-MS setup to provide high resolution as well as increased peak capacity.

The 2DLC is usually configured in either a heart-cutting mode or a comprehensive mode ${ }^{50}$ The heart-cutting analysis involves the collection of a few peaks of interest from the elution of the first dimension column, and then subjects only these peaks onto the second dimension column for further separation. This configuration can increase signal-to noise ratio and improve sensitivity for targeted metabolomics without extensive pre-sample preparation. ${ }^{51}$ In the comprehensive configuration, all eluents from the first dimension column are collected into multiple fractions and each fraction is subjected to the second dimension column for further separation. Collection of the fractions of elution from the first column and transferring them to the second column are done by an interface called a modulator. This is the key element of a two dimensional liquid chromatography system. The modulator consists of a switching valve and two storage loops. The comprehensive 
2DLC offers a higher resolution, so that more metabolite information can be obtained from the sample. ${ }^{52}$

Most of the 2DLC systems are operated in an online mode, where the second dimension separation is carried out simultaneously with the first dimension separation. ${ }^{53}$ Another form of
2DLC-MS gaining
popularity now is
called parallel
2DLC-MS, where
orthogonal HILIC
and RPC separation
occur in one
analytical run. In
parallel 2DLC-MS, $\begin{aligned} & \text { Figure 1.7. A sample 2DLC-MS instrumentation, the } \\ & \text { components of which are discussed below. (A) A Q-Exactive } \\ & \text { Mass Spectrometer with an electrospray source chamber. (B) A } \\ & \text { Thermo UltiMate 3000 Ultra Performance Liquid } \\ & \text { Chromatography with an UV detector, an auto sampler and a } \\ & \text { temperature controlled compartment containing the column. (C) } \\ & \text { Two pump that are used for two columns. }\end{aligned}$
a sample is first
delivered to two
sample loops during sample loading. ${ }^{54}$ The two sample aliquots are then simultaneously injected onto a dual column setup, and parallel separations are performed on the two columns. The eluates from the two columns are then merged and subjected to a mass spectrometer for further analysis. This strategy is simple yet effective for coupling HILIC and RPC for the purpose of decreasing analysis time and increasing throughput.

\subsection{Objectives of This Dissertation}


The first objective of this dissertation was to develop methods to increase the identification accuracy of metabolites in GC $\times$ GC-MS. The second objective of this dissertation was to integrate the multiple analytical platforms, i.e., GC×GC-MS and parallel 2DLC-MS, for analysis of same biological samples. Overall, this dissertation was devoted to solve the following problems encountered in untargeted metabolomics:

Poor accuracy of metabolite identification in GC×GC-MS. The accuracy of metabolite identification in GC $\times$ GC-MS using the first dimension retention index and mass spectrum matching is about $80-85 \%$, depending the identification algorithms. While the information of the second dimension retention time of each metabolite is readily available in GCXGCMS data, this information is not yet widely used in metabolomics to aid metabolite identification. The lack of using the second dimension retention index contributes to the poor accuracy of metabolite identification in GC×GC-MS based metabolomics.

\section{Large variation in calculating the second dimension retention index in GCXGC-MS.}

Isovolatility approach has been proposed to calculate the second dimension retention index

in $\mathrm{GC} \times \mathrm{GC}-\mathrm{MS}$. This process introduces large variation in the calculated second dimension retention index and prevents the use of the second dimension retention index for accurate metabolite identification. Furthermore, construction of a retention map using $n$-alkanes as reference compounds is not suitable for every column class. For instance, $n$-alkanes are nonpolar compounds and they do not have good retention in mid-polar and polar columns. Therefore, the retention map constructed using their retention times on either of these two column classes is very small. Moreover, several other problems are also associated with the second dimension retention index calculation, including that the current isovolatility curve approach approximates the second dimension separation as under pseudo-isothermal 
condition while the instrument actually operate under temperature-programmed mode; multiple data processing steps are involved in converting the second dimension retention time to the second dimension retention index; the retention index of a query compound is estimated using the isovolatility curves of two reference compounds; the public retention index database only contains limited retention index information, etc.

\section{High rate of false positive biomarker discovery in GC $\times$ GC-MS. Current GC×GC-MS}

based metabolomics performs metabolite identification solely depending on comparing the similarity of the first dimension retention index and EI mass spectrum between an experimental data and the information of compound standards. With the increase of the number of metabolites in the public metabolite database, a high rate of false metabolite identification occurs owing to the lack of using the second dimension retention index.

Limited metabolites coverage. Metabolite coverage in a particular analytical platform is determined by the separation technique and the detector used. For example, metabolites have to be volatile and thermally stable in order to be analyzed by GC-MS. Chemical derivatization can be opted to increase the coverage by derivatizing the metabolites thus making them volatile and thermally stable. Still, a huge number of compounds cannot be derivatized and therefore cannot be detected by GC-MS. In contrast to GC-MS, LC-MS can analyze non-volatile metabolites. However, the ionization technique used in LC-MS such as electron spray ionization (ESI) or matrix assisted laser induced ionization (MALDI) may have very low ionization efficiency for some metabolites and result that these metabolites cannot be detected. Moreover, metabolites need to be soluble in the solvents and mobile phase in order to be analyzed by LC-MS. 
In order to solve the above mentioned challenges encountered in untargeted metabolomics, several data analysis algorithms and bioanalytical methods have been developed in this dissertation. These developments have been organized in this dissertation as follows: Chapter Two introduces the development of a surface fitting method for calculating the second dimension retention index $\left({ }^{2} I\right)$. Chapter Three describes a further development in ${ }^{2} I$ calculation by removing the hold-up time calculation. Chapter Four presents a novel method of ${ }^{2} I$ calculation where mixtures of reference compounds are to allow the use of any compounds as reference compounds. Chapter Five demonstrates the analysis of metabolomic samples by multiple platforms for purposes of increased metabolite coverage, improved accuracy of metabolite quantification, and reliable metabolic pathway assignment. 


\section{CHAPTER 2}

\section{SURFACE FITTING FOR CALCULATING THE SECOND DIMENSION RETENTION INDEX IN COMPREHENSIVE TWO-DIMENSIONAL GAS CHROMATOGRAPHY MASS SPECTROMETRY}

\subsection{Introduction}

While mass spectrometry (MS) coupled with a one-dimensional separation technique, i.e., gas chromatography (GC) or liquid chromatography (LC), has been widely used in metabolomics, the limited separation power of one-dimensional GC or LC contributes to the poor performance of GC-MS and LC-MS in detecting low abundance compounds from complex samples, such as human body fluids and tissues. Comprehensive two-dimensional gas chromatography mass spectrometry (GC×GC-MS) uses two GC columns with different stationary phases to increase the separation power. The first dimension column, i.e., the primary column, is usually a 10 - 60 m long column while the second dimension column is a short column, about $1-2 \mathrm{~m} .{ }^{55-59}$ The two columns are connected by a thermal modulator. Compounds eluted from the first dimension column within a modulation period $P_{M}$ are collected by the thermal modulator and subjected to the second dimension column for further separation at an increased temperature. Depending on the system configuration, the modulation period is usually set $2-10 \mathrm{~s}$ and the temperature gradient on both columns 
is in a range of $5-10^{\circ} \mathrm{C} / \mathrm{min} .{ }^{60-64}$ The two columns of the GC $\times$ GC-MS are operated in linear temperature gradient condition. However, the fast separation and slow temperature gradient on the second dimension column result in a small temperature change within a $P_{M}$, making a condition such that the second dimension column separation treated as pseudo-isothermal condition. ${ }^{65-71} \mathrm{GC} \times \mathrm{GC}-\mathrm{MS}$ provides three pieces of information for each compound, the first dimension retention ${ }^{1} t_{R}$, the second retention time ${ }^{2} t_{R}$ and election ionization (EI) mass spectrum, if an EI source is used. ${ }^{38,39}$ To reduce the dependence of retention times on GC $\times$ GC-MS experimental conditions, ${ }^{1} t_{R}$ and ${ }^{2} t_{R}$ of a compound $u$ are respectively converted into linear retention index $I^{T}$ and Kovats retention index ${ }^{2} I .^{28,29}$

During GC $\times$ GC-MS analysis, compounds eluted within the same $\quad P_{M}$ from the first dimension column are subjected to the second dimension column for further separation at a different temperature, as well as on a different stationary phase. While the separation of the compounds on the second dimension column within one $P_{M}$ can be considered as in pseudo-isothermal condition, the overall temperature of the second dimension column, ${ }^{2} T_{e}$, changes during GC×GC-MS analysis. The retention time ${ }^{2} t_{R}$ of a compound in the second dimension column decreases with the increase of column temperature, ${ }^{2} T_{e}$. The relation $\left[{ }^{2} T_{R} \sim{ }^{2} T_{e}\right.$ ] of a compound is captured by an isovolatility curve. Several methods have been proposed to construct the isovolatility curves (using reference compounds such as $n$-alkanes) including Beens et al..$^{72,73}$, Marriot et al. ${ }^{40,41,74,75}$, and Zhao et al. ${ }^{76}$ In these reported methods, the hold-up time, ${ }^{2} t_{M}$, at different temperatures must be first estimated to get the adjusted retention time ${ }^{2} t_{R}^{\prime}$. The ${ }^{2} t_{R}^{\prime}$ values of the same reference compound at 
different column temperatures were then used to construct the isovolatility curves of the reference compounds. The isovolatility curves of all reference compounds formed the second dimension retention index map, and the ${ }^{2} I$ value of a compound was finally calculated from the two adjacent isovolatility curves of two reference compounds.

While several methods have been developed to calculate the ${ }^{2} I$ of compounds, however ${ }^{2} I$ has not been extensively used for compound identification due to the inaccuracy of ${ }^{2} I$ calculation. It should be noted that a few papers used the seconddimension retention index to aid compound identification in analysis of GC×GC-MS data. $^{39,77}$ However, from our experience, the variation window of the second-dimension retention index used in these papers are much smaller and the credibility of these papers is questionable. Inaccuracy of ${ }^{2} I$ calculation arise from the isovolatility curve construction step as different fitting method will provide different results. For instance, the isovolatility curve in Figure 1.5 can be constructed by polynomial fitting, logarithmic fitting, exponential fitting, etc. Different fitting will give different ${ }^{2} I$ values for a particular compound.

The objective of this study was to develop a method to calculate the ${ }^{2} I$ value of each compound without construction of an isovolatility curves. We developed a surface fitting method for the calculation of ${ }^{2} I$ values for all compounds detected by GC $\times$ GC-MS. The developed surface fitting method used $n$-alkanes as reference compounds, where the second dimension retention time, ${ }^{2} t_{R}$, and the second dimension column temperature, ${ }^{2} T_{e}$, formed a $X$ - $Y$ plane and the ${ }^{2} I$ was the $Z$-axis to form the ${ }^{2} I$ surface. The robustness of the surfacing fitting method was validated using 10 -fold cross validation. The method 
was then applied to calculate the retention indices of 152 compound standards in two commercially available mixtures, MegaMix A and MegaMix B.

\subsection{Experimental}

\subsubsection{Chemicals}

A mixture containing $\mathrm{C}_{7}-\mathrm{C}_{30} n$-alkanes was purchased from Sigma-Aldrich Corp., St. Louis, MO. MegaMix A (Cat. No. 8270) and MegaMix B (Cat. No. 8260) were purchased from Restek Corp., Bellefonte, PA. All of the compounds were present at a purity of between $95-99 \%$. Two solutions were prepared from these three commercial mixtures. One solution contained only $\mathrm{C}_{7}-\mathrm{C}_{30} n$-alkanes, while the other contained $\mathrm{C}_{7}-\mathrm{C}_{30} n$-alkanes, MegaMix A and MegaMix B. These two solutions were prepared in dichloromethane at a concentration of $25 \mu \mathrm{g} / \mathrm{mL}$ per compound.

\subsubsection{GCXGC-MS Experiment}

The LECO (St. Joseph, MI) Pegasus ${ }^{\circledR}$ 4D GC $\times$ GC-MS instrument was equipped with a Gerstel MPS2 autosampler and an Agilent 6890 gas chromatograph featuring a LECO two-stage cryogenic modulator and a secondary oven. The first dimension column was a non-polar DB-5ms (phenyl arylene polymer virtually equivalent to a (5\%-phenyl)methylpolysiloxane) $60.0 \mathrm{~m} \times 0.25 \mathrm{~mm}{ }^{1} d_{c} \times 0.25 \mu \mathrm{m}^{1} d_{f}$ column, and the second dimension column was a medium polar BD-17ms ((50\%-phenyl)-methylpolysiloxane) 1.0 $\mathrm{m} \times 0.1 \mathrm{~mm}{ }^{2} d_{c} \times 0.1 \mu \mathrm{m}^{2} d_{f}$ column. Both columns were obtained from Agilent Technologies (Santa Clara, CA), and were connected by means of a press-fit connector before the thermal modulator. The flow rate of ultra-high purity helium carrier gas 
(99.999\%) was set to $2.0 \mathrm{~mL} / \mathrm{min}$ at a corrected constant flow via pressure ramps with an initial head pressure of 36.9 psi.

A $1 \mu \mathrm{L}$ sample was injected into the inlet chamber of the GC×GC-MS system and the split ratio was set at $20: 1$. The inlet temperature was $280{ }^{\circ} \mathrm{C}$. The secondary oven and the thermal modulator were respectively set to $+5{ }^{\circ} \mathrm{C}$ and $+15{ }^{\circ} \mathrm{C}$ relative to the primary oven. The MS parameters were: MS range $45-800 \mathrm{~m} / \mathrm{z}$; data acquisition rate $200 \mathrm{spectra} / \mathrm{s}$; temperature of ion source chamber $230{ }^{\circ} \mathrm{C}$; temperature of MS transfer line $280{ }^{\circ} \mathrm{C}$; detector voltage $1641 \mathrm{~V}$; electron energy $70 \mathrm{eV}$. The acceleration voltage was turned on after a solvent delay of $220 \mathrm{~s}$.

The mixture containing $\mathrm{C}_{7}-\mathrm{C}_{30} n$-alkanes was analyzed on $\mathrm{GC} \times \mathrm{GC}-\mathrm{MS} 46$ times under isothermal conditions. Temperature of the first dimension column, ${ }^{l} T_{e}$, was set at 60 ${ }^{\circ} \mathrm{C}$ in the first injection, and increased $+5{ }^{\circ} \mathrm{C}$ in each of the following 45 injections with ${ }^{1} T_{e}$ $=285^{\circ} \mathrm{C}$ in the last injection. The second dimension column was also set under isothermal conditions but at $+5{ }^{\circ} \mathrm{C}$ with respect to the first dimension column. $P_{M}$ was set to $10 \mathrm{~s}$.

MegaMix A, MegaMix B and $\mathrm{C}_{7}-\mathrm{C}_{30} n$-alkanes were mixed as one solution, and then analyzed four times in temperature programed conditions with the first dimension column temperature initially set as $60,80,100$, and $120^{\circ} \mathrm{C}$, respectively. The column temperature was held at the initial temperature for $1 \mathrm{~min}$ and then ramped at $5{ }^{\circ} \mathrm{C} / \mathrm{min}$ to $285^{\circ} \mathrm{C}$, and kept at $285^{\circ} \mathrm{C}$ for $13 \mathrm{~min}$. The secondary column temperature program was identical to that of the first dimension column but temperature was set $+5{ }^{\circ} \mathrm{C}$ higher than the first dimension column. $P_{M}$ was set as $4 \mathrm{~s}$.

\subsubsection{Spectrum Deconvolution and Compound Identification}


The LECO ChromaTOF software (version 4.51), equipped with the National Institute of Standards and Technology MS database (NIST 2014), was used for instrument control, spectrum deconvolution, and compound identification. Manufacturer recommended parameters for ChromaTOF were used to reduce the raw instrument data into a peak list of compounds. These parameters were: baseline offset 0.5 ; auto smoothing; peak width in first dimension $6 \mathrm{~s}$; peak width in the second dimension $0.1 \mathrm{~s}$; signal-to-noise ratio 100; match required to combine peaks 600 ; R.T. shift $0.08 \mathrm{~s}$; minimum forward similarity match 600. The true peak spectrum was exported as part of the information for each chromatographic peak as absolute intensity values.

\subsubsection{Hold-up Time Calculation}

To calculate the hold-up time on the second dimension column ${ }^{2} t_{M}, n$-alkane mixture was analyzed on GC×GC-MS at 46 different column temperatures in isothermal conditions. Hold-up time ${ }^{2} t_{M}$ was then calculated as follows ${ }^{76}$

$$
2 t_{M}=\frac{t_{R 1} t_{R 3}-t_{R 2}^{2}}{\left(t_{R 3}-t_{R 2}\right)-\left(t_{R 2}-t_{R 1}\right)}
$$

where $t_{R 1}, t_{R 2}$ and $t_{R 3}$ are the continuous retention times of three homologs such as $C_{n-1}$, $C_{n}$ and $C_{n+1}$ alkanes. 
Multiple $n$-alkanes were analyzed on the second dimension column at the same column temperature. Therefore, multiple hold-up times were obtained for each temperature using equation (3). The mean values of hold-up time at different temperatures were then fitted by a function (Figure 2.1) and the form of the function is as follows:

$$
{ }^{2} \mathrm{t}_{\mathrm{M}}=-0.06000 \ln \left({ }^{2} \mathrm{~T}_{\mathrm{e}}\right)+1.194
$$

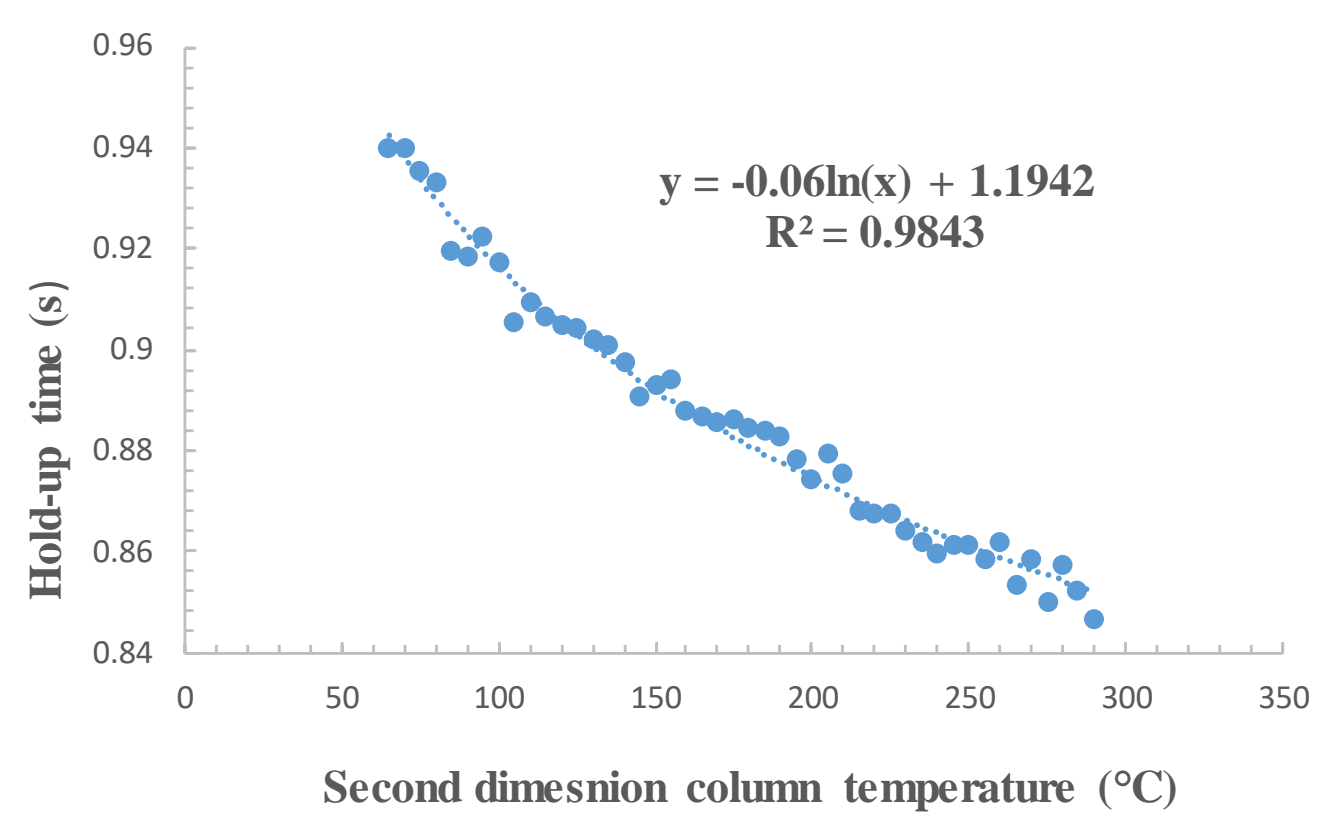

Figure 2.1. The second dimension hold-up time calculation. The means of hold-up times at each column temperature were fitted using a logarithm function.

where ${ }^{2} t_{M}$ is the fitted hold-up time in the second dimension column and ${ }^{2} \mathrm{~T}_{\mathrm{e}}$ is the second dimension column temperature in ${ }^{\circ} \mathrm{C}$. The fitting accuracy $R^{2}$ was 0.9843 .

\subsubsection{Construction of the Second Dimension Retention Index Surface}

$\mathrm{C}_{7}-\mathrm{C}_{30} n$-alkanes were analyzed 46 times by GC×GC-MS in isothermal conditions. ${ }^{2} t_{R}$ and ${ }^{2} T_{e}$ were obtained for each reference compound in each analysis. The ${ }^{2} I$ value 
of a reference compound with $n$ carbon atoms was defined as $100 \times n$. Therefore, each $n$ alkane compound was represented by $\left\{{ }^{2} t_{R}^{\prime},{ }^{2} T_{e},{ }^{2} I\right\}$. A second dimension retention index surface ${ }^{2} I_{S}$ was then constructed using the data of all $n$-alkane compounds, where ${ }^{2} t_{R}^{\prime}$ and ${ }^{2} T_{e}$ formed the $X$ - $Y$ plane and the ${ }^{2} I$ was the $Z$-axis to form the ${ }^{2} I_{S}$. A polynomial surface model in MATLAB (Mathworks, Natick, MA) named 'polyij' was used to construct ${ }^{2} I_{S}$, where $i$ was the degree of polynomial in ${ }^{2} t_{R}^{\prime}$, and $j$ was the degree of polynomial in ${ }^{2} T_{e}$. The maximum for both $i$ and $j$ was five in the MATLAB platform. The degree of polynomial was the maximum of $i$ and $j$.

\subsubsection{Evaluation Criteria of Surface Fitting Method}

Three measures were used to evaluate the quality of surface fitting, including $R$-square $\left(R^{2}\right)$, degrees of freedom adjusted R-square $\left(R_{a d j}^{2}\right)$, and root mean squared error (RMSE). $R^{2}$ measures how successful the fit is in explaining the variation of the data, and is defined as the ratio of the sum of squares of the regression $(S S R)$ and the total sum of squares $(S S T)$.

$$
\begin{aligned}
& S S R=\sum_{i=1}^{n} w_{i}(\hat{y}-\bar{y})^{2} \\
& S S T=\sum_{i=1}^{n} w_{i}\left(y_{i}-\bar{y}\right)^{2} \\
& R^{2}=\frac{\operatorname{SSR}}{\operatorname{SST}}
\end{aligned}
$$

where $y_{i}$ is the original retention index value ${ }^{2} I_{i}, \hat{y}$ is the predicted ${ }^{2} I$ value, $\bar{y}$ is the mean of the original retention index values, and the $w_{i}$ is the weight on the value ${ }^{2} I_{i} \cdot R^{2}$ can take on any value between 0 and 1 , with a value closer to 1 indicating that a greater proportion of variance is accounted for by the model. 
$R_{a d j}^{2}$ adjusts $R^{2}$ based on the residual degrees of freedom $v$ that is defined as the number of response values, $n$, minus the number of fitted coefficients, $m$, estimated from the response values. The $R_{a d j}^{2}$ can take on any value less than or equal to 1 , with a value closer to 1 indicating a better fit.

$$
R_{a d j}^{2}=1-\frac{\operatorname{SSE}(n-1)}{\operatorname{SST}(v)}
$$

where $S S E$ is defined as

$$
S S E=\sum_{i=1}^{n} w_{i}\left(y_{i}-\hat{y}\right)^{2}
$$

$R M S E$ is an estimate of the standard deviation of the random component in the data, and is defined as

$$
R M S E=\sqrt{M S E}=\sqrt{\frac{S S E}{v}}
$$

where $M S E$ is the mean square error or the residual mean square. An $R M S E$ value closer to 0 indicates a fit that is more useful for prediction.

\subsection{Results and Discussion}

$\mathrm{C}_{7}-\mathrm{C}_{30} n$-alkanes were chosen as the reference compounds to create a retention index surface, ${ }^{2} I_{S}$, in this study. All $n$-alkanes were identified from the GC×GC-MS data. A total of 102 compounds were identified from the mixture of MegaMix A and MegaMix B.

\subsubsection{GC $\times$ GC-MS Experimental Variability}


The mixture of $n$-alkanes was analyzed in temperature-programmed mode 25 times in five continuous days. For the experiments performed within one day, the largest standard deviation of the second dimension retention time of $n$-alkanes is 0.007 s. Figure 2.2 displays the cumulative probability curves of the day-to-day experiments, i.e. 25 replicates analyzed in five days.

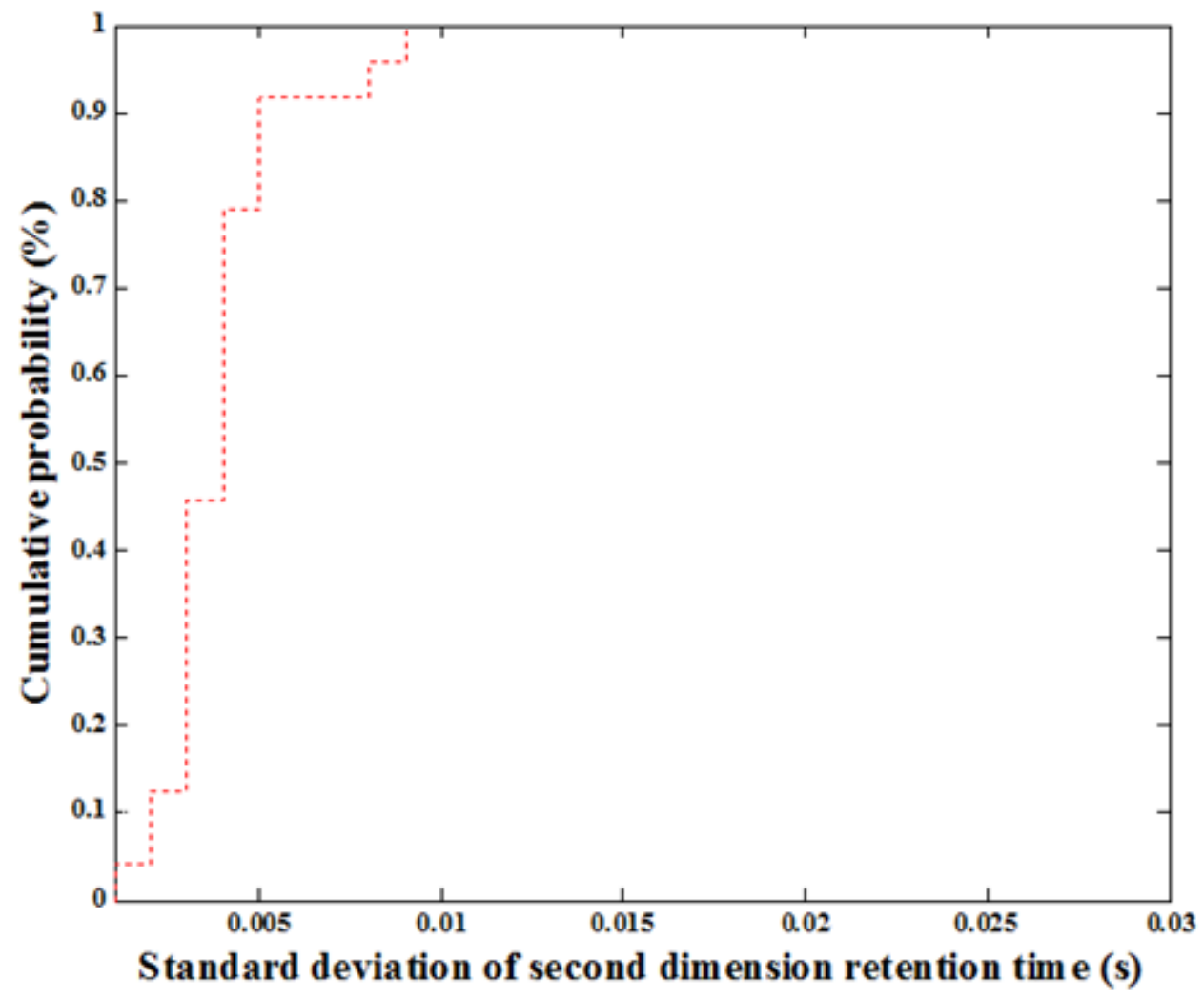

Figure 2.2. Cumulative probability curves of standard deviation of the second dimension retention times of $n$-alkanes.

The standard deviation of the second dimension retention time of the $n$-alkanes fluctuated from $0.001 \mathrm{~s}$ to $0.007 \mathrm{~s}$. The standard deviation of $90 \%$ of the second dimension retention time values in all 25 experiments were less than $0.005 \mathrm{~s}$. Such a small standard deviation of the second dimension retention time indicates that the instrument has a very 
good reproducibility in measuring the retention times of compounds separated on the second dimension column in temperature-programmed mode.

While the $n$-alkane mixture was analyzed in temperature-programmed mode, each chromatographic run was ramped to $285^{\circ} \mathrm{C}$ in the first dimension column and held at $285^{\circ} \mathrm{C}$ for $13 \mathrm{~min}$. By design, the second dimension column was in fact operated in isothermal mode at $290{ }^{\circ} \mathrm{C}$ during the last $13 \mathrm{~min}$ in each of the 25 experiments. Five $n$ alkanes, i.e. $\mathrm{C}_{26}-\mathrm{C}_{30} n$-alkanes, were eluted in isothermal mode in the second dimension column at $290{ }^{\circ} \mathrm{C}$. The standard deviation of the second dimension retention times of these five $n$-alkanes were $0.004,0.004,0.004,0.004$ and $0.005 \mathrm{~s}$, respectively. Such small standard deviations indicate that the reproducibility of the GC $\times$ GC-MS system is also quite good in isothermal mode.

\subsubsection{Construction of the Second Dimension Retention Index Surface}

A total of 415 data points of $\mathrm{C}_{7}-\mathrm{C}_{30} n$-alkanes were used to construct the second dimension retention surface, ${ }^{2} I_{S}$. To obtain the optimal surface fitting, all combinations of $i$ and $j$ values in the polynomial surface model 'poly $i j$ ' were tested. The performance of each polynomial surface model was then evaluated Table 2.1.

Table 2.1. Selection of polynomial surface model 'polyij' to construct the second dimension retention index surface using GC×GC-MS data of $n$-alkanes (weight ' $y$ ' and robust 'LAR')

\begin{tabular}{ccccc}
\hline Polyij & & $\mathrm{R}^{2}$ & $R_{a d j}^{2}$ & $R M S E$ \\
& & & & \\
\hline $\mathrm{I}$ & $\mathrm{j}$ & & & \\
\hline 1 & 1 & 0.955 & 0.9547 & 148.7 \\
2 & 1 & 0.9984 & 0.9984 & 27.8
\end{tabular}




\begin{tabular}{ccccc}
3 & 1 & 0.9972 & 0.9972 & 37 \\
4 & 1 & 0.9961 & 0.996 & 44.2 \\
5 & 1 & 0.995 & 0.9949 & 49.8 \\
1 & 2 & 0.999 & 0.999 & 22.4 \\
2 & 2 & 0.9995 & 0.9995 & 16 \\
3 & 2 & 0.9991 & 0.999 & 21.7 \\
4 & 2 & 0.9986 & 0.9985 & 26.9 \\
5 & 2 & 0.9982 & 0.9981 & 30.4 \\
1 & 3 & 0.9987 & 0.9987 & 25.3 \\
2 & 3 & 0.9997 & 0.9997 & 11.3 \\
3 & 3 & 0.9997 & 0.9997 & 12.2 \\
4 & 3 & 0.9995 & 0.9995 & 15.6 \\
5 & 3 & 0.9994 & 0.9993 & 18.1 \\
1 & 4 & 0.9984 & 0.9983 & 28.5 \\
2 & 4 & 0.9999 & 0.9999 & 7.8 \\
3 & 4 & 0.9995 & 0.9995 & 15.7 \\
4 & 4 & 0.9995 & 0.9995 & 15.7 \\
5 & 4 & 0.9998 & 0.9998 & 10.4 \\
1 & 5 & 0.9978 & 0.9977 & 33.4 \\
2 & 5 & 0.9999 & 0.9999 & 6.1 \\
3 & 5 & 0.9999 & 0.9999 & 6.3 \\
4 & 5 & 0.9999 & 0.9999 & 6.7 \\
5 & 5 & 0.9999 & 0.9999 & 6.8 \\
\hline
\end{tabular}

The optimal polynomial model in this study was 'poly25', i.e. $i=2$ and $j=5$. The optimal model was selected based on the three evaluation criteria. $R^{2}, R_{a d j}^{2}$, and $R M S E$ of the optimal surface fitting model 'poly 25 ' were $0.9999,0.9999$ and 6.1 iu, respectively. Tenfold cross validation was performed to study how accurately the polynomial surface model 'poly 25 ' would perform in fitting the $n$-alkane data points. The original 415 data points were randomly partitioned into 10 subsamples, where 9 subsamples had equal sample size (42 data points each) and the $10^{\text {th }}$ subsample had 37 data points. Of the 10 subsamples, a single subsample was retained as the validation data for testing the model, and the 
remaining 9 subsamples were used as training data. Figure 2.3 is an example showing that the validation data set was randomly distributed.

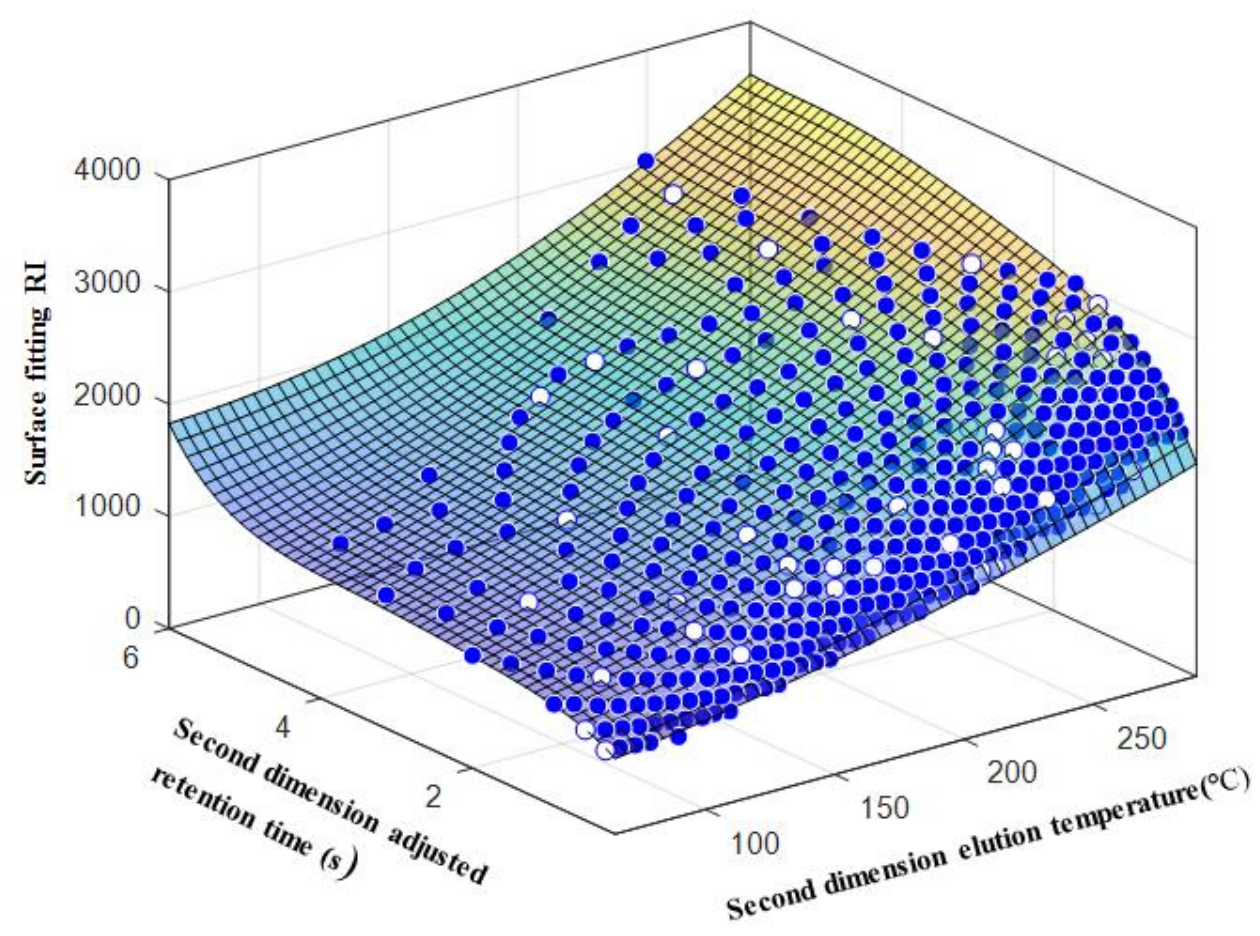

Figure 2.3. Distribution of one validation data set used in 10 -fold cross validation. The filled circles are the training data set and the open circles are the validation data set.

The cross validation process was repeated 10 times, with each of the 10 subsamples used exactly once as the validation data. The 10 validation results were then averaged to produce a single estimation. Table 2.2 lists the results of the 10 -fold cross validation. The $R^{2}, R_{a d j}^{2}$, and $R M S E$ of the training data set were $0.9999,0.9999$ and 7.1 iu, respectively. 
The average $R M S E$ for the validation set of data was $16.0 \mathrm{iu}$. The 10 -fold cross validation process was repeated three more time, and the Table 2.3 shows the results of the second repeated trial. Third and fourth repeated trial's average $R M S E$ for the validation set of data was $15.8 \mathrm{iu}$ and $15.7 \mathrm{iu}$ respectively (data not shown). The similar results of the four trials demonstrate that the polynomial surface model 'poly25' was very robust in fitting the $n$ alkane data points.

Table 2.2. Results of a ten-fold cross validation of surface fitting using the second dimension retention indices of $n$-alkanes

\begin{tabular}{ccccc}
\hline Fold count & \multicolumn{2}{c}{ Goodness of fit } & & Goodness of validation \\
\hline & $R^{2}$ & $R^{2}{ }_{a d j}$ & $R M S E$ & $R M S E$ \\
\hline 1 & 0.9999 & 0.9999 & 6.3 & 17.2 \\
2 & 0.9999 & 0.9999 & 6.2 & 15.7 \\
3 & 0.9999 & 0.9999 & 6.3 & 17.3 \\
4 & 0.9999 & 0.9999 & 6.3 & 17.1 \\
5 & 0.9999 & 0.9999 & 10.1 & 16.0 \\
6 & 0.9999 & 0.9999 & 6.3 & 16.4 \\
7 & 0.9999 & 0.9999 & 10.6 & 14.5 \\
8 & 0.9999 & 0.9999 & 6.4 & 17.3 \\
9 & 0.9999 & 0.9999 & 6.1 & 17.0 \\
10 & 0.9999 & 0.9999 & 6.4 & 11.9 \\
Average & 0.9999 & 0.9999 & 7.1 & 16.0 \\
\hline
\end{tabular}


Table 2.3. Results of a ten-fold cross validation of surface fitting using the second dimension retention indices of $n$-alkanes (repeated trial)

\begin{tabular}{|c|c|c|c|c|}
\hline \multirow[t]{2}{*}{ Fold Count } & \multicolumn{3}{|c|}{ Goodness of fit } & \multirow{2}{*}{$\begin{array}{c}\text { Goodness of validation } \\
R M S E\end{array}$} \\
\hline & $\mathrm{R}^{2}$ & $R_{a d j}^{2}$ & $R M S E$ & \\
\hline 1 & 0.9999 & 0.9999 & 6.4 & 10.2 \\
\hline 2 & 0.9999 & 0.9999 & 6.4 & 13.2 \\
\hline 3 & 0.9999 & 0.9999 & 6.4 & 13.7 \\
\hline 4 & 0.9999 & 0.9999 & 6.3 & 16.5 \\
\hline 5 & 0.9999 & 0.9999 & 6.2 & 21.2 \\
\hline 6 & 0.9999 & 0.9999 & 6.2 & 15.7 \\
\hline 7 & 0.9999 & 0.9999 & 6 & 21.4 \\
\hline 8 & 0.9999 & 0.9999 & 6.4 & 11.6 \\
\hline 9 & 0.9999 & 0.9999 & 62 & 19.5 \\
\hline 10 & 0.9999 & 0.9999 & 6.1 & 20.5 \\
\hline Average & 0.9999 & 0.9999 & 6.2 & 16.3 \\
\hline
\end{tabular}

Figure 2.4 (A) shows the second dimension retention index surface, ${ }^{2} I_{S}$, constructed from the isothermal data of the $n$-alkanes using polynomial surface fitting model 'poly 25 '. Figure 2.4(B) is the residual plot of the surface fitting. A residual from the fitted surface model was defined as the difference between the original retention index ${ }^{2} I$ of an $n$-alkane and its fitted value. The residuals had a near normal distribution (Figure 2.4(C)), suggesting that no significant bias was introduced by the polynomial surface model 'poly 25 '. However, Figure 2.4. B indicates large residuals showed at the lower end of the $Y$-axis, which were mainly caused by the inaccuracy of chromatographic peak picking and the small values of ${ }^{2} t_{R}$ of $n$-alkanes. Small variation in determining an $n$-alkane's ${ }^{2} t_{R}$ during peak picking resulted in large variation in positioning the data point of that $n$-alkane in the $X-Y$ plane. 
A.

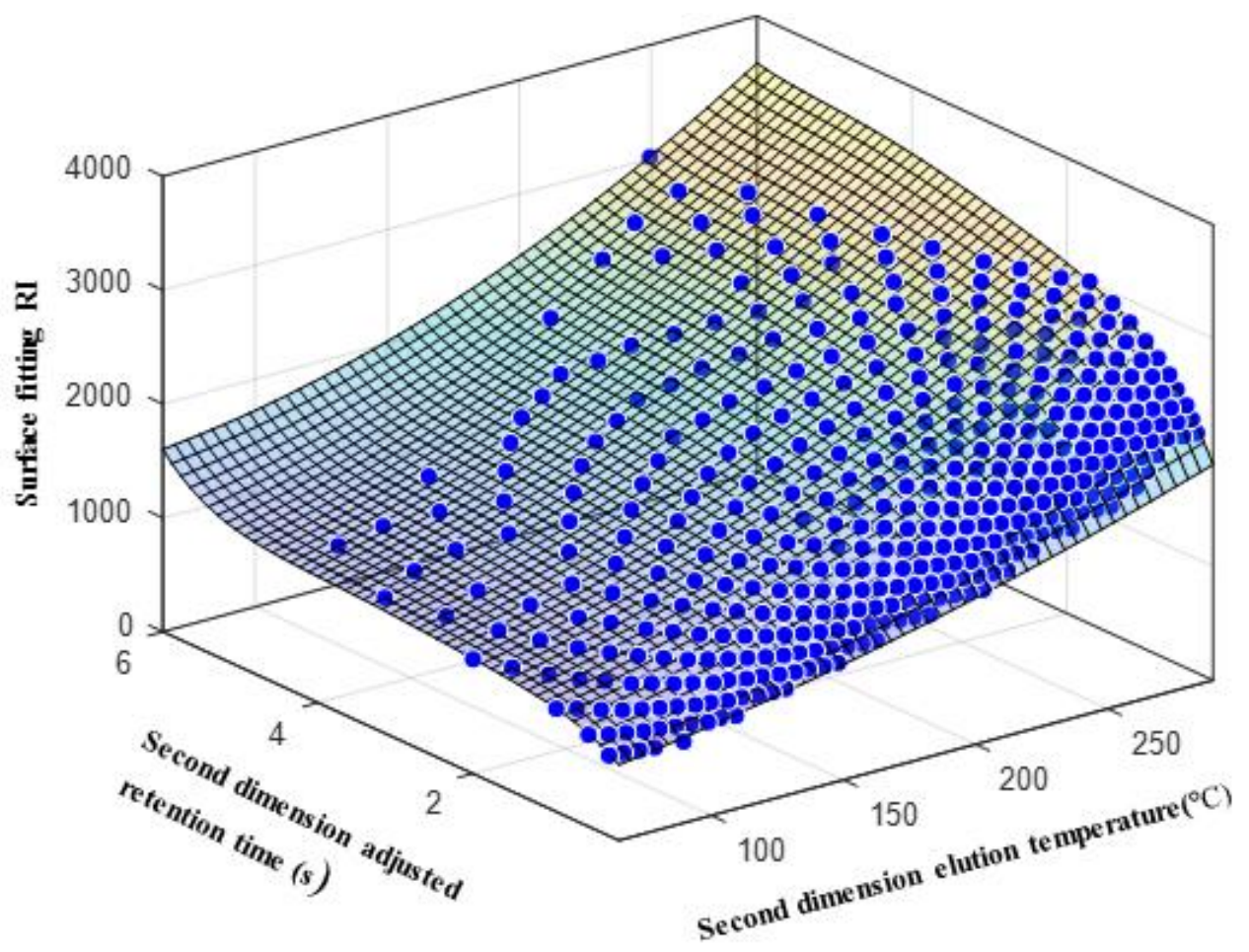

B.

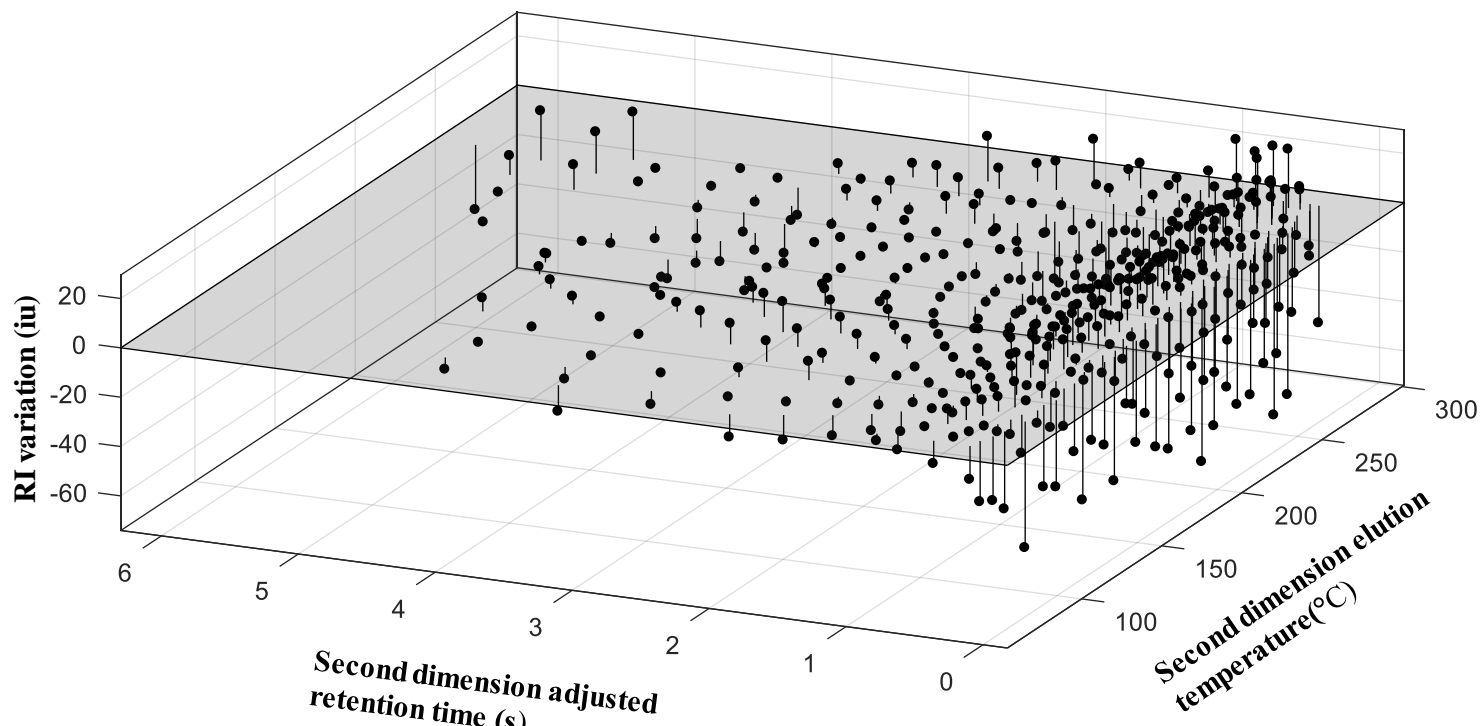


C.

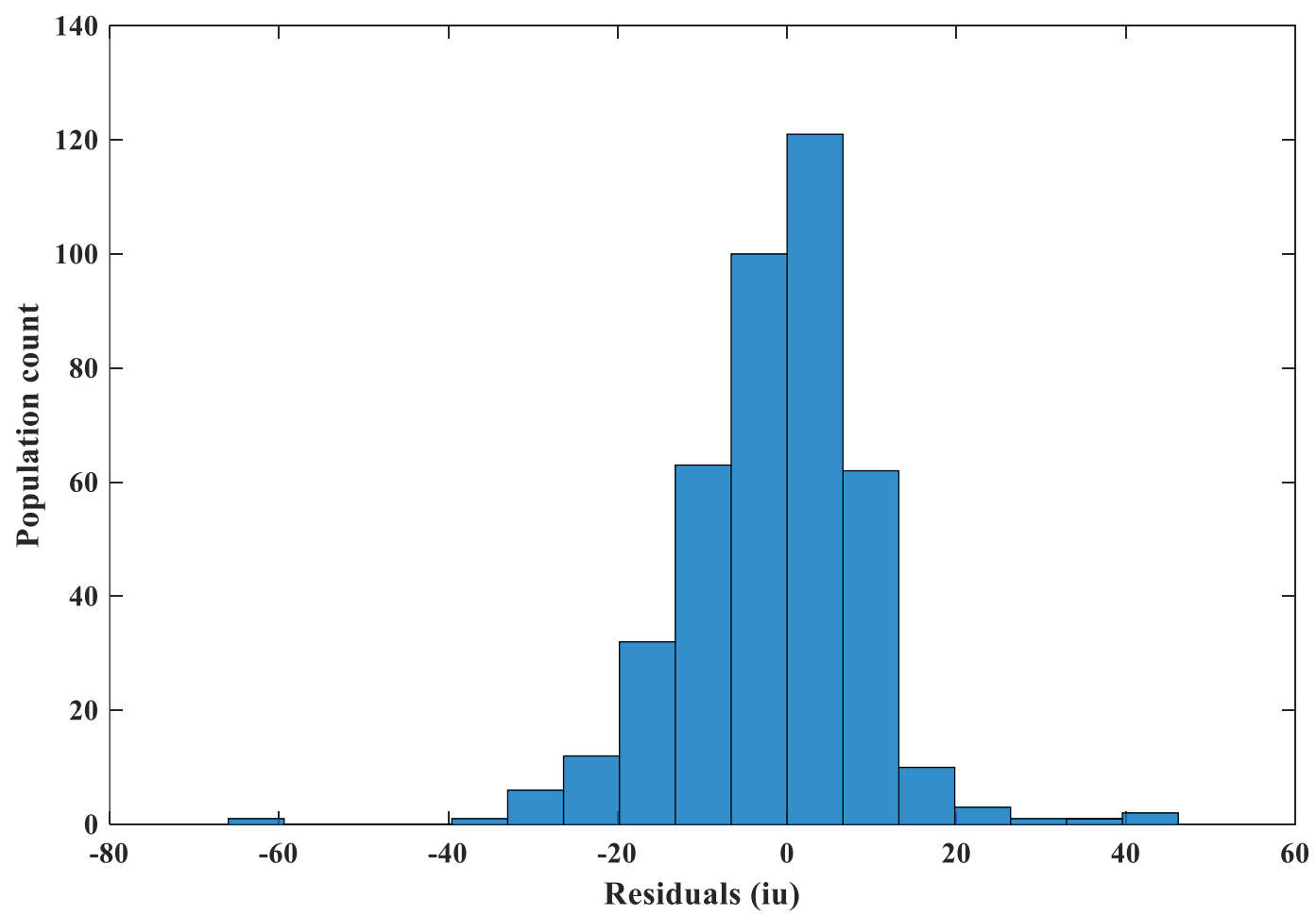

Figure 2.4. The second dimension retention index surface constructed using the $\mathrm{GC} \times \mathrm{GC}-$ MS data of $n$-alkanes. The retention index surface is a function of the elution temperature of each $n$-alkane in the second dimension column ${ }^{2} T_{e}$ and the corresponding adjusted retention time ${ }^{2} t_{R}^{\prime}$. (A) is the second dimension retention index surface. (B) is the residual plot, and $(\mathrm{C})$ is the distribution of the residuals.

\subsubsection{Calculation of Retention Index of Compounds in MegaMix A \& MegaMix B}

MegaMix A, MegaMix B, and $\mathrm{C}_{7}-\mathrm{C}_{30} n$-alkanes were mixed and analyzed by GC $\times$ GC-MS in temperature programed conditions with the first dimension column started at 4 different initial temperatures, 60, 80, 100 and $120{ }^{\circ} \mathrm{C}$, respectively. After data processing, 68 compounds were identified from MegaMix A and 41 compounds were identified from MegaMix B. Seven compounds were identified from both MegaMix A and 
MegaMix B. Pyridine is one of the 76 compounds in the Megamix A. While pyridine was identified from the experiment data, it was excluded from the retention index calculation because it is one of the carry-over compounds frequently detected in our GCxGC-MS system. Tables 2.4 and 2.5 list the ${ }^{2} I$ values of compounds identified from MegaMix A and MegaMix B, respectively. The standard deviation of ${ }^{2} I$ calculated from the four analyses ranged from 1 to $10 \mathrm{iu}$. The mean standard deviation of ${ }^{2} I$ was 1.6 iu for compounds in MegaMix A and 3.4 iu for compounds in MegaMix B. The average RSD for MegaMix A and MegaMix B was $0.10 \%$ and $0.31 \%$, respectively.

Table 2.4. The second dimension retention indices of compounds identified from MegaMix A

\begin{tabular}{|c|c|c|c|c|c|c|}
\hline \multirow{2}{*}{ Compound name } & \multicolumn{4}{|c|}{$\begin{array}{l}\text { Second dimension retention index } \\
\text { (iu })^{\mathrm{a}}\end{array}$} & \multirow{2}{*}{$\begin{array}{l}\text { Mean } \\
\text { (iu) }\end{array}$} & \multirow{2}{*}{$\begin{array}{l}\text { STD } \\
\text { (iu) }\end{array}$} \\
\hline & $65^{\circ} \mathrm{C}$ & $85^{\circ} \mathrm{C}$ & $105^{\circ} \mathrm{C}$ & $125^{\circ} \mathrm{C}$ & & \\
\hline N-Nitrosodimethylamine & 983 & 977 & 977 & & 979 & 3 \\
\hline Aniline & 1250 & 1252 & 1250 & 1249 & 1250 & 1 \\
\hline Phenol & 1205 & 1202 & 1198 & 1199 & 1201 & 3 \\
\hline Bis(2-chloroethyl) ether & 1218 & 1215 & 1211 & 1208 & 1213 & 4 \\
\hline 2-Chlorophenol & 1217 & 1213 & 1216 & 1219 & 1216 & 2 \\
\hline Benzyl alcohol & 1297 & 1299 & 1300 & & 1298 & 1 \\
\hline $\begin{array}{l}\text { 2,2'-Oxybis }(2- \\
\text { chloropropane })\end{array}$ & 1247 & 1245 & 1242 & 1241 & 1244 & 3 \\
\hline 2-Methylphenol & 1290 & 1290 & 1288 & 1286 & 1288 & 2 \\
\hline N-nitrosodipropylamine & 1304 & 1302 & 1305 & 1295 & 1301 & 4 \\
\hline 3-Methylphenol & 1312 & 1313 & 1315 & 1309 & 1312 & 2 \\
\hline Hexachloroethane & 1275 & 1275 & 1272 & 1277 & 1275 & 2 \\
\hline
\end{tabular}




\begin{tabular}{|c|c|c|c|c|c|}
\hline Isophorone & 1373 & 1374 & 1373 & 1373 & 1373 \\
\hline 2-Nitrophenol & 1417 & 1420 & 1423 & 1426 & 1421 \\
\hline 2,4-Dimethylphenol & 1396 & 1396 & 1396 & 1394 & 1396 \\
\hline Bis(2-chloroethoxy)-methane & 1422 & 1423 & 1422 & 1418 & 1421 \\
\hline 2,4-DichloroPhenol & 1435 & 1434 & 1435 & 1436 & 1435 \\
\hline 4-Chloroaniline & 1537 & 1538 & 1542 & 1548 & 1541 \\
\hline 4-Chloro-3-methylphenol & 1587 & 1586 & 1586 & 1590 & 1588 \\
\hline 2-Methylnaphthalene & 1608 & 1607 & 1610 & 1614 & 1610 \\
\hline 1-Methylnaphthalene & 1640 & 1642 & 1644 & 1649 & 1644 \\
\hline $\begin{array}{l}\text { 1,3-Cyclopentadiene, } \\
\text { 1,2,3,4,5,5-hexachloro- }\end{array}$ & 1573 & 1572 & 1571 & 1572 & 1572 \\
\hline 2,4,6-Trichlorophenol & 1665 & 1664 & 1666 & 1667 & 1666 \\
\hline 2,4,5-Trichlorophenol & 1673 & 1673 & 1672 & 1678 & 1674 \\
\hline 2-Chloronaphthalene & 1725 & 1726 & 1728 & 1732 & 1728 \\
\hline 2-Nitroaniline & 1834 & 1835 & 1837 & 1842 & 1837 \\
\hline 1,4-Dinitrobenzene & 1875 & 1875 & 1876 & 1879 & 1876 \\
\hline 1,3-Dinitrobenzene & 1886 & 1886 & 1887 & 1890 & 1887 \\
\hline Dimethyl phthalate & 1842 & 1842 & 1842 & 1846 & 1843 \\
\hline 2,6-Dinitrotoluene & 1883 & 1882 & 1883 & 1887 & 1884 \\
\hline 1,2-Dinitrobenzene & 1931 & 1929 & 1931 & 1933 & 1931 \\
\hline Acenapthylene & 1851 & 1851 & 1852 & 1857 & 1853 \\
\hline 3-Nitroaniline & 1951 & 1950 & 1951 & 1957 & 1952 \\
\hline Acenaphthene & 1883 & 1883 & 1884 & 1887 & 1884 \\
\hline 2,4-dinitro-phenol & 1944 & 1943 & 1941 & 1944 & 1943 \\
\hline Dibenzofuran & 1914 & 1914 & 1914 & 1919 & 1915 \\
\hline 2,4-Dinitrotoluene & 1968 & 1966 & 1966 & 1967 & 1967 \\
\hline 2,3,5,6-Tetrachlorophenol & 1922 & 1922 & 1921 & 19223 & 1922 \\
\hline 2,3,4,6-Tetrachlorophenol & 1936 & 1935 & 1936 & 1937 & 1936 \\
\hline
\end{tabular}




$\begin{array}{lllllll}\text { Diethyl Phthalate } & 1984 & 1982 & 1981 & 1982 & 1982 & 1 \\ \text { Fluorene } & 2007 & 2007 & 2007 & 2009 & 2008 & 1 \\ \text { 4-Chlorophenyl phenyl ether } & 1979 & 1979 & 1978 & 1980 & 1979 & 1 \\ \text { 4-Nitroaniline } & 2141 & 2141 & 2140 & 21411 & 2141 & 1 \\ \text { 4,6-Dinitro-2-methylphenol } & 2050 & 2050 & 2049 & 2050 & 2050 & 1 \\ \text { Diphenylamine } & 2062 & 2061 & 2063 & 2063 & 2062 & 1 \\ \text { Azobenzene } & 2034 & 2034 & 2034 & 2035 & 2034 & 0 \\ \text { 4-Bromophenyl phenyl ether } & 2122 & 2120 & 2121 & 2122 & 2121 & 1 \\ \text { Hexachlorobenzene } & 2112 & 2109 & 2112 & 2112 & 2111 & 1 \\ \text { Pentachloro-phenol } & 2202 & 2199 & 2201 & 2200 & 2201 & 1 \\ \text { Anthracene } & 2298 & 2298 & 2298 & 2299 & 2298 & 0 \\ \text { Phenanthrene } & 2308 & 2305 & 2308 & 2309 & 2307 & 1 \\ \text { Carbazole } & 2412 & 2412 & 2410 & 2412 & 2411 & 1 \\ \text { di-n-Butyl phthalate } & 2370 & 2370 & 2369 & 2368 & 2369 & 1 \\ \text { Fluoranthene } & 2676 & 2677 & 2676 & 2676 & 2676 & 1 \\ \text { Pyrene } & 2772 & 2772 & 2771 & 2773 & 2772 & 1 \\ \text { Benzyl butyl phthalate } & 2930 & 2931 & 2929 & 2927 & 2929 & 1 \\ \text { Bis(2-ethylhexyl) adipate } & 2642 & 2644 & 2640 & 2644 & 2643 & 2 \\ \text { Benz[a]anthracene } & 3198 & 3199 & 3200 & 3197 & 3199 & 1 \\ \text { Chrysene } & 3231 & 3231 & 3231 & 3230 & 3231 & 1 \\ \text { Bis(2-ethylhexyl) phthalate } & 2929 & 2927 & 2931 & 2931 & 2929 & 1 \\ \text { di-n-Octyl phthalate } & 3076 & 3081 & 3074 & 3079 & 3077 & 3\end{array}$

${ }^{a}$ The blank means the compound was not detected during the experiment.

Table 2.5. The second dimension retention indices of compounds identified from MegaMix B 


\begin{tabular}{|c|c|c|c|c|c|c|}
\hline \multirow{2}{*}{ Compound name } & \multicolumn{4}{|c|}{$\begin{array}{l}\text { Second dimension retention index } \\
\qquad(\mathrm{iu})^{\mathrm{a}}\end{array}$} & \multirow{2}{*}{$\begin{array}{l}\text { Mean } \\
\text { (iu) }\end{array}$} & \multirow{2}{*}{$\begin{array}{l}\text { STD } \\
\text { (iu) }\end{array}$} \\
\hline & $65^{\circ} \mathrm{C}$ & $85^{\circ} \mathrm{C}$ & $105^{\circ} \mathrm{C}$ & $125^{\circ} \mathrm{C}$ & & \\
\hline $\begin{array}{l}\text { cis-1,3-Dichloro-1- } \\
\text { Propene, }(\mathrm{Z})\end{array}$ & 888 & 891 & & & 889 & 2 \\
\hline $\begin{array}{l}\text { trans-1,3-Dichloro-Propene } \\
\text { (E) }\end{array}$ & 927 & 924 & & & 925 & 1 \\
\hline Toluene & 899 & & & & 899 & 0 \\
\hline 1,1,2-Trichloroethane & 937 & 941 & & & 939 & 2 \\
\hline Ethyl methacrylate & 906 & 915 & & & 910 & 5 \\
\hline 1,3-Dichloropropane & 953 & 949 & 965 & & 956 & 7 \\
\hline Dibromochloro-methane & 987 & 984 & 994 & & 988 & 4 \\
\hline Tetrachloroethene & 926 & 931 & 950 & & 936 & 10 \\
\hline 1,2-Dibromoethane & 1000 & 994 & 1006 & & 1000 & 5 \\
\hline Chlorobenzene & 1015 & 1012 & 1021 & & 1016 & 4 \\
\hline $1,1,1,2$-Tetrachloroethane & 10011 & 1002 & & & 1019 & 5 \\
\hline Ethylbenzene & 1007 & 1005 & & & 1006 & 1 \\
\hline p-Xylene & 1014 & 1015 & 1019 & & 1016 & 2 \\
\hline Styrene & 1072 & 1070 & 1075 & 1075 & 1073 & 2 \\
\hline o-Xylene & 1054 & 1052 & 1058 & 1046 & 1052 & 4 \\
\hline Bromoform & 1128 & 1125 & 1125 & 1140 & 1130 & 6 \\
\hline $\begin{array}{l}\text { cis-1,4-Dichloro-2-butene, } \\
\text { (z)- }\end{array}$ & 1114 & 1111 & 1106 & 1117 & 1112 & 4 \\
\hline 1,1,2,2-Tetrachloroethane & 1118 & 1114 & 1110 & 1119 & 1115 & 4 \\
\hline 1,2,3-Trichloropropane & 1139 & 1136 & 1131 & 1135 & 1135 & 3 \\
\hline Isopropylbenzene(cumene) & 1072 & 1070 & 1069 & 1088 & 1075 & 8 \\
\hline $\begin{array}{l}\text { trans-1,4-Dichloro-2- } \\
\text { butene, }(\mathrm{E}) \text { - }\end{array}$ & 1152 & 1149 & 1139 & 1147 & 1147 & 5 \\
\hline Bromobenzene & 1140 & 1138 & 1135 & 1145 & 1139 & 4 \\
\hline
\end{tabular}




\begin{tabular}{|c|c|c|c|c|c|c|}
\hline 2-Chlorotoluene & 1141 & 1140 & & & 1141 & 0 \\
\hline n- Propylbenzene & 1109 & 1107 & 1105 & & 1107 & 2 \\
\hline 4-Chlorotoluene & 1142 & 1141 & 1140 & 1149 & 1143 & 3 \\
\hline 1,3,5-Trimethylbenzene & 1122 & 1120 & 1120 & 1131 & 1123 & 4 \\
\hline Pentachloroethane & 1171 & 1168 & 1165 & 1181 & 1171 & 6 \\
\hline tert-Butylbenzene & 1148 & 1146 & 1146 & 1151 & 1148 & 2 \\
\hline 1,2,4-Trimethylbenzene & 1163 & 1160 & 1156 & 1167 & 1162 & 4 \\
\hline sec-Butylbenzene & 1167 & 1167 & 1164 & 1168 & 1166 & 1 \\
\hline $\begin{array}{l}\text { p-Cymene(4-isopropyl } \\
\text { toluene) }\end{array}$ & 1182 & 1178 & 1177 & 1186 & 1181 & 4 \\
\hline n-Butylbenzene & 1220 & 1219 & 1214 & 1217 & 1218 & 3 \\
\hline $\begin{array}{l}\text { 1,2-Dibromo-3- } \\
\text { Chloropropane }\end{array}$ & 1363 & 1366 & 1365 & 1368 & 1366 & 2 \\
\hline Benzene, 1,2,3-trichloro- & 1493 & 1494 & 1497 & 1499 & 1496 & 2 \\
\hline 1,3-Dichlorobenzene ${ }^{*}$ & 1216 & 1215 & 1214 & & 1215 & 1 \\
\hline 1,4-Dichlorobenzene* & 1231 & 1230 & 1227 & 1213 & 1225 & 7 \\
\hline 1,2-Dichlorobenzene* & 1266 & 1267 & 1268 & 1269 & 1267 & 1 \\
\hline Nitrobenzene* & 1380 & 1385 & 1388 & 1390 & 1386 & 4 \\
\hline Benzene, 1,2,4-trichloro-* & 1434 & 1435 & 1436 & 1436 & 1435 & 1 \\
\hline Naphthalene* & 1485 & 1487 & 1489 & 1495 & 1489 & 4 \\
\hline \multicolumn{7}{|l|}{ 1,3-Butadiene, 1,1,2,3,4,4- } \\
\hline hexachloro-* & 1416 & 1417 & 1415 & 1415 & 1416 & 1 \\
\hline
\end{tabular}

${ }^{a}$ The blank means the compound was not detected during the experiment.

Compared with the compounds in MegaMix A, the relatively increased mean standard deviation of ${ }^{2} I$ of the compounds in MegaMix B may be related with the retention times of the compounds. The compounds in MegaMix A eluted in the middle to high temperature 
range whereas the compounds in MegaMix B eluted in the low to mid temperature range. To an individual compound, the large standard deviation of ${ }^{2} I$ was mainly caused by the inaccuracy of peak picking, during which the second dimension retention time ${ }^{2} t_{R}$ of the compound was determined. For instance, the standard deviation in ${ }^{2} I$ of compound 1, 4dochlorobenzene was 7 iu. While 1, 4-dichlorobenzene was well separated from other compounds during the analysis with the initial temperature of the first dimension column set at $60{ }^{\circ} \mathrm{C}$, its chromatographic peak overlapped with the chromatographic peaks of other compounds during the analysis when the initial temperature of the first dimension column was at $125^{\circ} \mathrm{C}$ (Figure 2.5).

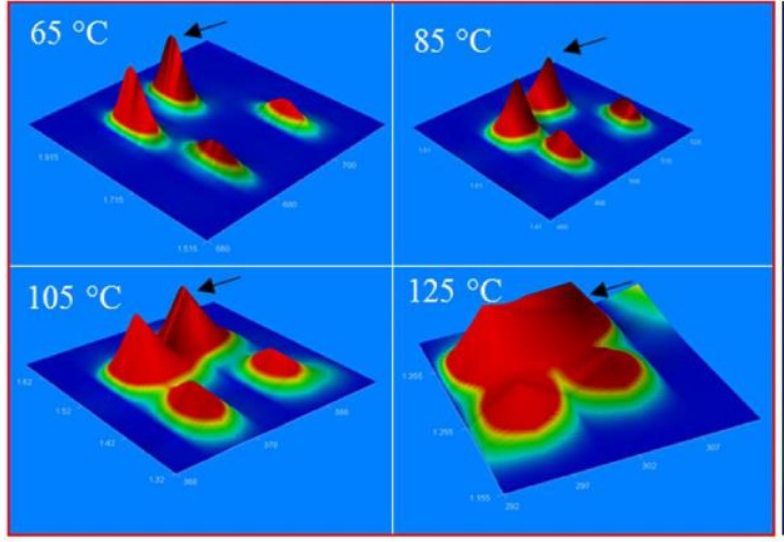

(A)

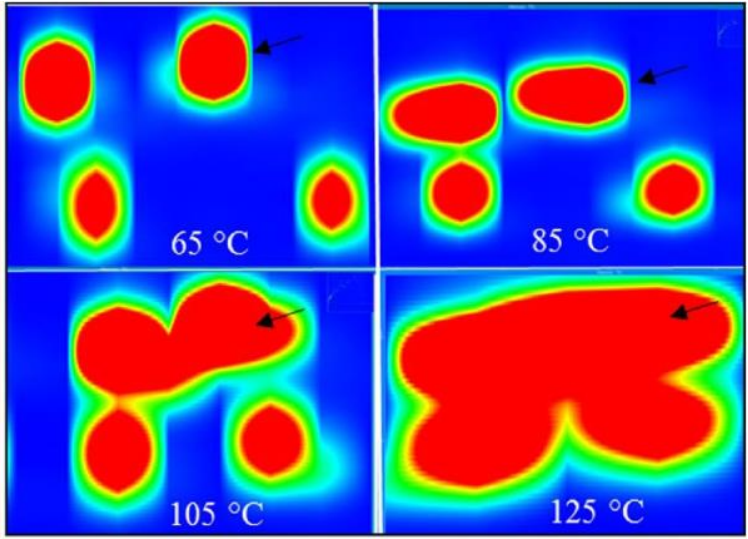

(B)

Figure 2.5. GC $\times$ GC-MS separation of 1, 4-dichlorobenzene (black arrow) and other compounds in MegaMix A analyzed in temperature-programmed mode at different column temperatures. With the increase of the second dimension column temperature from $65^{\circ} \mathrm{C}$ to $125^{\circ} \mathrm{C}$, the separation between 1,4 -dichlorobenzene and other compounds became worse and resulted in chromatographic peak overlapping. The overlapping chromatographic peaks introduce a high degree of variation in the retention time and 
therefore the calculated retention index. (A) are the three-dimensional images of the two dimensional chromatographic peaks, and (B) are the corresponding contour images.

Inaccuracy of deconvoluting overlapping chromatographic peaks increased the chance of introducing large variation in determining a compound's second dimension retention time ${ }^{2} t_{R}$ and therefore its ${ }^{2} I$ value. Tables 2.6 and 2.7 list the second dimension column temperature ${ }^{2} T_{e}$ and corresponding adjusted second dimension retention time ${ }^{2} t_{R}^{\prime}$ of each identified compound in MegaMix A and MegaMix B, respectively.

The average range of elution temperature of compounds in MegaMix A is $8{ }^{\circ} \mathrm{C}$, and $25{ }^{\circ} \mathrm{C}$ for compounds in MegaMix B. Compared with compounds in MegaMix A, compounds in MegaMix B had larger ${ }^{2} I$ variations (Table 2.5) and their elution temperature ranges were also quite broader (Table 2.7) than those of the compounds in MegaMix A (Tables 2.4 and 2.6).

Previous study demonstrated that retention index had temperature dependency, i.e. retention index of an analyte in general increases with the increase of elution temperature 78. We observed that some of the identified compounds followed this trend in this study, while other compounds did not. The reason is that temperature dependency of retention index is also related to the structure of an analyte. ${ }^{78-81}$ 
Table 2.6. Second dimension elution temperature $\left({ }^{2} T_{e}\right)$ of MegaMix A compounds at different initial temperature with ramp 5

${ }^{\circ} \mathrm{C} / \mathrm{min}$ and the corresponding adjusted second dimension retention time.

\begin{tabular}{|c|c|c|c|c|c|c|c|c|}
\hline \multirow[t]{2}{*}{ Compounds name } & \multicolumn{4}{|c|}{$\begin{array}{l}{ }^{2} T_{e}\left({ }^{\circ} \mathrm{C}\right) \text { at different initial temperature with ramp } \\
5^{\circ} \mathrm{C} / \mathrm{min}\end{array}$} & \multicolumn{4}{|c|}{$\begin{array}{l}\text { Adjusted }{ }^{2} t_{R}(\mathrm{~s}) \text { at different initial temperature } \\
\text { with ramp } 5^{\circ} \mathrm{C} / \mathrm{min}\end{array}$} \\
\hline & $65^{\circ} \mathrm{C}$ & $85^{\circ} \mathrm{C}$ & $105^{\circ} \mathrm{C}$ & $125^{\circ} \mathrm{C}$ & $65^{\circ} \mathrm{C}$ & $85^{\circ} \mathrm{C}$ & $105^{\circ} \mathrm{C}$ & $125^{\circ} \mathrm{C}$ \\
\hline N-Nitrosodimethylamine & 90.5 & 106.1 & 123.8 & & 0.711 & 0.446 & 0.260 & \\
\hline Aniline & 116.9 & 123.2 & 133.8 & 148.1 & 1.341 & 1.095 & 0.795 & 0.541 \\
\hline Phenol & 116.8 & 122.5 & 133.1 & 147.4 & 1.056 & 0.879 & 0.644 & 0.440 \\
\hline Bis(2-chloroethyl) ether & 117.8 & 123.8 & 134.1 & 148.1 & 1.097 & 0.900 & 0.665 & 0.451 \\
\hline 2-Chlorophenol & 119.2 & 124.8 & 135.1 & 149.1 & 1.043 & 0.865 & 0.660 & 0.461 \\
\hline Benzyl alcohol & 125.2 & 129.5 & 138.1 & & 1.286 & 1.123 & 0.871 & \\
\hline $\begin{array}{l}2,2^{\prime} \text {-Oxybis }(2- \\
\text { chloropropane) }\end{array}$ & 127.5 & 131.5 & 139.5 & 151.4 & 0.937 & 0.829 & 0.657 & 0.477 \\
\hline 2-Methylphenol & 127.5 & 131.2 & 139.1 & 151.1 & 1.147 & 1.023 & 0.807 & 0.582 \\
\hline N-nitrosodipropylamine & 130.2 & 133.5 & 140.8 & 152.5 & 1.128 & 1.009 & 0.828 & 0.582 \\
\hline 3-Methylphenol & 130.8 & 133.8 & 140.8 & 152.1 & 1.148 & 1.050 & 0.863 & 0.622 \\
\hline Hexachloroethane & 131.8 & 135.8 & 142.8 & 154.1 & 0.939 & 0.835 & 0.683 & 0.518 \\
\hline Isophorone & 138.2 & 140.8 & 146.8 & 156.5 & 1.207 & 1.113 & 0.925 & 0.714 \\
\hline 2-Nitrophenol & 139.9 & 142.5 & 148.2 & 157.5 & 1.402 & 1.298 & 1.096 & 0.849 \\
\hline 2,4-Dimethylphenol & 141.5 & 143.5 & 148.2 & 157.1 & 1.203 & 1.129 & 0.981 & 0.759 \\
\hline Bis(2-chloroethoxy)- & & & & & & & & \\
\hline Methane & 143.2 & 145.2 & 149.8 & 158.1 & 1.279 & 1.204 & 1.036 & 0.810 \\
\hline 2,4-DichloroPhenol & 147.5 & 149.2 & 153.5 & 160.1 & 1.175 & 1.111 & 0.983 & 0.820 \\
\hline 4-Chloroaniline & 150.5 & 151.9 & 155.5 & 162.5 & 1.687 & 1.612 & 1.439 & 1.176 \\
\hline 4-Chloro-3-methylphenol & 162.5 & 162.9 & 164.8 & 169.5 & 1.376 & 1.356 & 1.272 & 1.119 \\
\hline 2-Methylnaphthalene & 164.2 & 164.9 & 167.2 & 172.2 & 1.422 & 1.382 & 1.298 & 1.130 \\
\hline 1-Methylnaphthalene & 166.5 & 167.2 & 169.2 & 173.8 & 1.498 & 1.473 & 1.389 & 1.220 \\
\hline
\end{tabular}




\begin{tabular}{|c|c|c|c|c|c|c|c|c|}
\hline $1,2,3,4,5,5$-hexachloro- & 167.8 & 168.5 & 170.5 & 174.8 & 1.103 & 1.078 & 1.014 & 0.906 \\
\hline 2,4,6-Trichlorophenol & 171.5 & 171.9 & 173.2 & 176.8 & 1.399 & 1.380 & 1.330 & 1.191 \\
\hline 2,4,5-Trichlorophenol & 172.9 & 173.2 & 174.5 & 177.8 & 1.380 & 1.365 & 1.306 & 1.202 \\
\hline 2-Chloronaphthalene & 175.2 & 175.9 & 177.2 & 180.5 & 1.566 & 1.536 & 1.481 & 1.348 \\
\hline -Nitroaniline & 178.3 & 178.6 & 179.9 & 182.6 & 2.182 & 2.162 & 2.072 & 1.918 \\
\hline ,4-Dinitrobenzene & 181.6 & 181.9 & 182.9 & 185.2 & 2.263 & 2.238 & 2.163 & 2.014 \\
\hline ,3-Dinitrobenzene & 183.6 & 183.6 & 184.6 & 186.9 & 2.199 & 2.194 & 2.129 & 1.975 \\
\hline Dimethyl phthalate & 182.9 & 183.2 & 184.2 & 186.2 & 1.893 & 1.873 & 1.809 & 1.709 \\
\hline 2,6-Dinitrotoluene & 184.3 & 184.2 & 185.2 & 187.2 & 2.119 & 2.114 & 2.044 & 1.930 \\
\hline 1,2-Dinitrobenzene & 185.3 & 185.6 & 186.3 & 188.3 & 2.444 & 2.399 & 2.359 & 2.215 \\
\hline Acenapthylene & 184.9 & 185.2 & 186.2 & 188.5 & 1.824 & 1.804 & 1.749 & 1.640 \\
\hline 3-Nitroaniline & 188.6 & 188.6 & 189.3 & 190.9 & 2.335 & 2.330 & 2.280 & 2.196 \\
\hline Acenaphthene & 189.2 & 189.2 & 190.2 & 192.2 & 1.770 & 1.765 & 1.716 & 1.616 \\
\hline 2,4-dinitro-phenol & 191.9 & 191.9 & 192.2 & 193.6 & 2.016 & 2.011 & 1.976 & 1.902 \\
\hline Dibenzofuran & 193.2 & 193.5 & 194.2 & 195.9 & 1.727 & 1.707 & 1.667 & 1.602 \\
\hline ,4-Dinitrotoluene & 193.6 & 193.9 & 194.2 & 195.6 & 2.077 & 2.042 & 2.017 & 1.927 \\
\hline$, 3,5,6$-Tetrachlorophenol & 195.9 & 195.9 & 196.2 & 197.9 & 1.617 & 1.622 & 1.598 & 1.523 \\
\hline ,3,4,6-Tetrachlorophenol & 196.9 & 196.9 & 197.5 & 198.5 & 1.648 & 1.638 & 1.608 & 1.563 \\
\hline Diethyl Phthalate & 199.9 & 200.2 & 200.5 & 201.2 & 1.759 & 1.729 & 1.704 & 1.669 \\
\hline $\begin{array}{l}\text { Fluorene } \\
\text { 4-Chlorophenyl phenyl }\end{array}$ & 201.6 & 201.6 & 201.9 & 203.2 & 1.799 & 1.804 & 1.779 & 1.715 \\
\hline ther & 201.9 & 202.2 & 202.5 & 203.5 & 1.614 & 1.599 & 1.574 & 1.535 \\
\hline -Nitroaniline & 203.6 & 203.6 & 204.0 & 204.3 & 2.645 & 2.650 & 2.605 & 2.590 \\
\hline methylphenol & 203.6 & 203.6 & 203.9 & 204.6 & 1.950 & 1.950 & 1.920 & 1.885 \\
\hline Diphenylamine & 204.9 & 204.9 & 205.2 & 206.2 & 1.940 & 1.935 & 1.925 & 1.861 \\
\hline Azobenzene & 205.6 & 205.9 & 205.9 & 206.9 & 1.725 & 1.705 & 1.705 & 1.656 \\
\hline ther & 213.6 & 213.6 & 213.9 & 214.5 & 1.768 & 1.753 & 1.738 & 1.708 \\
\hline Hexachlorobenzene & 214.2 & 214.2 & 214.5 & 215.2 & 1.673 & 1.658 & 1.653 & 1.618 \\
\hline
\end{tabular}




\begin{tabular}{|c|c|c|c|c|c|c|c|c|}
\hline Pentachloro-phenol & 220.2 & 220.2 & 220.2 & 220.6 & 1.824 & 1.809 & 1.819 & 1.795 \\
\hline Anthracene & 224.3 & 224.3 & 224.3 & 224.6 & 2.151 & 2.151 & 2.151 & 2.131 \\
\hline Phenanthrene & 225.3 & 225.2 & 225.6 & 225.9 & 2.141 & 2.126 & 2.121 & 2.101 \\
\hline Carbazole & 230.3 & 229.9 & 229.9 & 230.3 & 2.467 & 2.492 & 2.477 & 2.467 \\
\hline di-n-Butyl phthalate & 239.5 & 239.5 & 239.5 & 239.5 & 1.620 & 1.620 & 1.615 & 1.610 \\
\hline Fluoranthene & 252.3 & 252.3 & 252.3 & 252.6 & 2.478 & 2.483 & 2.478 & 2.448 \\
\hline Pyrene & 257.6 & 257.6 & 257.6 & 257.6 & 2.659 & 2.659 & 2.654 & 2.669 \\
\hline Benzyl butyl phthalate & 274.9 & 274.9 & 274.9 & 274.9 & 2.278 & 2.283 & 2.273 & 2.263 \\
\hline Bis(2-ethylhexyl) adipate & 277.8 & 277.8 & 277.8 & 277.8 & 1.128 & 1.133 & 1.123 & 1.133 \\
\hline Benz[a]anthracene & 286.0 & 286.0 & 286.0 & 286.0 & 2.955 & 2.960 & 2.965 & 2.950 \\
\hline Chrysene & 287.0 & 287.0 & 287.0 & 287.0 & 3.070 & 3.070 & 3.070 & 3.060 \\
\hline Bis(2-ethylhexyl) & & & & & & & & \\
\hline phthalate & 289.2 & 289.2 & 289.2 & 289.2 & 1.516 & 1.511 & 1.521 & 1.521 \\
\hline di-n-Octyl phthalate & 290.0 & 290.0 & 290.0 & 290.0 & 2.011 & 2.031 & 2.001 & 2.026 \\
\hline
\end{tabular}

Table 2.7. Second dimension elution temperature $\left({ }^{2} T_{e}\right)$ of MegaMix B compounds at different initial temperature with ramp 5

${ }^{\circ} \mathrm{C} / \mathrm{min}$ and the corresponding adjusted second dimension retention time.

\begin{tabular}{|c|c|c|c|c|c|c|c|c|}
\hline \multirow[t]{2}{*}{ Compound name } & \multicolumn{4}{|c|}{$\begin{array}{l}{ }^{2} T_{e}\left({ }^{\circ} \mathrm{C}\right) \text { at different initial } \\
\text { temperature with ramp } 5{ }^{\circ} \mathrm{C} / \mathrm{min}\end{array}$} & \multicolumn{4}{|c|}{$\begin{array}{l}\text { Adjusted }{ }^{2} t_{R}(\mathrm{~s}) \text { at different initial } \\
\text { temperature with ramp } 5^{\circ} \mathrm{C} / \mathrm{min}\end{array}$} \\
\hline & $65^{\circ} \mathrm{C}$ & $85^{\circ} \mathrm{C}$ & $105^{\circ} \mathrm{C}$ & $125^{\circ} \mathrm{C}$ & $65^{\circ} \mathrm{C}$ & $85^{\circ} \mathrm{C}$ & $105^{\circ} \mathrm{C}$ & $125^{\circ} \mathrm{C}$ \\
\hline cis-1,3-Dichloro-1-Propene, $(\mathrm{Z})$ & 90.8 & 106.4 & & & 0.401 & 0.251 & & \\
\hline trans-1,3-Dichloro-Propene $€$ & 92.8 & 107.1 & & & 0.483 & 0.311 & & \\
\hline Toluene & 93.1 & & & & 0.403 & & & \\
\hline 1,1,2-Trichloroethane & 93.5 & 107.8 & & & 0.503 & 0.342 & & \\
\hline Ethyl methacrylate & 93.8 & 107.8 & & & 0.413 & 0.287 & & \\
\hline
\end{tabular}


1,3-Dichloropropane

Dibromochloro-Methane

Tetrachloroethene

1,2-Dibromoethane

Chlorobenzene

1,1,1,2-Tetrachloroethane

Ethylbenzene

p-Xylene

Styrene

0-Xylene

Bromoform

cis-1,4-Dichloro-2-butene, , (z)-

1,1,2,2-Tetrachloroethane

1,2,3-Trichloropropane

Isopropylbenzene(cumene)

trans-1,4-Dichloro-2-butene, (E)-

Bromobenzene

2-Chlorotoluene

n- Propylbenzene

4-Chlorotoluene

1,3,5-Trimethylbenzene

Pentachloroethane

tert-Butylbenzene

1,2,4-Trimethylbenzene

p-Cymene(4-isopropyl toluene)

n-Butylbenzene

n-Butylbenzene

1,2-Dibromo-3-Chloropropane

Benzene, 1,2,3-trichloro-

1,3-Dichlorobenzene*

1,4-Dichlorobenzene*

\begin{tabular}{|c|c|c|c|c|c|c|c|}
\hline 94.5 & 108.1 & 124.8 & & 0.539 & 0.357 & 0.230 & \\
\hline 96.1 & 109.4 & 125.4 & & 0.620 & 0.423 & 0.276 & \\
\hline 96.4 & 109.4 & 125.8 & & 0.435 & 0.303 & 0.196 & \\
\hline 97.1 & 110.1 & 125.8 & & 0.650 & 0.438 & 0.296 & \\
\hline 100.8 & 112.1 & 127.1 & & 0.638 & 0.459 & 0.311 & \\
\hline 101.1 & 112.4 & & & 0.618 & 0.429 & & \\
\hline 102.1 & 113.1 & & & 0.588 & 0.430 & & \\
\hline 103.1 & 113.8 & 128.1 & & 0.594 & 0.445 & 0.297 & \\
\hline 105.8 & 115.5 & 129.1 & 145.4 & 0.745 & 0.566 & 0.397 & 0.265 \\
\hline 105.8 & 115.5 & 129.1 & 144.8 & 0.680 & 0.516 & 0.362 & 0.199 \\
\hline 106.2 & 115.8 & 129.5 & 145.4 & 0.991 & 0.736 & 0.508 & 0.350 \\
\hline 107.2 & 116.1 & 129.4 & 145.4 & 0.891 & 0.681 & 0.463 & 0.310 \\
\hline 108.8 & 117.5 & 130.1 & 145.8 & 0.867 & 0.667 & 0.463 & 0.310 \\
\hline 109.8 & 118.1 & 130.8 & 146.1 & 0.938 & 0.727 & 0.503 & 0.335 \\
\hline 109.8 & 118.1 & 130.8 & 146.1 & 0.668 & 0.527 & 0.368 & 0.255 \\
\hline 109.8 & 118.1 & 130.5 & 146.1 & 1.003 & 0.772 & 0.528 & 0.355 \\
\hline 111.2 & 119.5 & 131.5 & 146.8 & 0.903 & 0.708 & 0.504 & 0.345 \\
\hline 113.8 & 121.1 & & & 0.840 & 0.684 & & \\
\hline 113.8 & 121.1 & 132.4 & & 0.720 & 0.584 & 0.424 & \\
\hline 114.8 & 121.8 & 133.1 & 147.4 & 0.820 & 0.674 & 0.494 & 0.345 \\
\hline 115.8 & 122.5 & 133.4 & 147.8 & 0.726 & 0.599 & 0.444 & 0.311 \\
\hline 117.5 & 124.1 & 134.5 & 148.8 & 0.877 & 0.715 & 0.535 & 0.391 \\
\hline 119.1 & 125.1 & 135.1 & 148.8 & 0.748 & 0.631 & 0.480 & 0.336 \\
\hline 119.5 & 125.5 & 135.5 & 149.1 & 0.798 & 0.666 & 0.500 & 0.361 \\
\hline 123.8 & 128.8 & 137.5 & 150.4 & 0.770 & 0.662 & 0.521 & 0.382 \\
\hline 121.8 & 127.1 & 136.5 & 149.8 & 0.759 & 0.657 & 0.506 & 0.356 \\
\hline 128.5 & 132.5 & 140.1 & 152.1 & 0.807 & 0.719 & 0.572 & 0.422 \\
\hline 133.5 & 136.8 & 143.8 & 154.5 & 1.344 & 1.221 & 0.979 & 0.738 \\
\hline 146.8 & 148.8 & 153.2 & 161.1 & 1.195 & 1.131 & 0.998 & 0.801 \\
\hline 121.5 & 127.1 & 136.5 & & 0.969 & 0.817 & 0.631 & \\
\hline 122.8 & 128.1 & 137.1 & 149.8 & 0.999 & 0.852 & 0.656 & 0.441 \\
\hline
\end{tabular}




\begin{tabular}{|lrrrrrrrr} 
1,2-Dichlorobenzene* & 125.5 & 130.2 & 138.8 & 151.5 & 1.091 & 0.948 & 0.747 & 0.537 \\
Nitrobenzene* & 133.2 & 136.9 & 143.5 & 154.5 & 1.484 & 1.331 & 1.089 & 0.803 \\
Benzene, 1,2,4-trichloro-* & 151.5 & 153.2 & 156.8 & 164.2 & 1.332 & 1.268 & 1.139 & 0.932 \\
Naphthalene* & 148.5 & 150.2 & 154.5 & 162.2 & 1.421 & 1.357 & 1.188 & 0.971 \\
$\begin{array}{l}\text { 1,3-Butadiene, 1,1,2,3,4,4-hexachloro- } \\
*\end{array}$ & 151.5 & 152.8 & 156.5 & 163.5 & 0.967 & 0.933 & 0.839 & 0.697 \\
\hline
\end{tabular}




\subsubsection{Robustness of Surface Fitting to Variations of Instrumental Settings}

To investigate the robustness of the developed surface fitting method in analyzing GC $\times$ GC-MS data, we analyzed the mixture containing MegaMix A and MegaMix B in different instrumental conditions, by varying the temperature offset of the secondary oven, the ramp rate of temperature gradient, and modulation period. Tables 2.8 and 2.9 list the second dimension retention indices of compounds identified from MegaMix A and MegaMix B in different instrumental conditions, respectively.

An average relative standard deviation $(R S D)$ of the calculated second dimension retention index ${ }^{2} I$ for compounds in MegaMix A was $0.63 \%$ and $0.42 \%$ for compounds in MegaMix B. The small $R S D$ of calculated ${ }^{2} I$ demonstrates that the developed surface fitting method is robust in calculating the second dimension retention index of compounds analyzed in different instrumental conditions.

As we analyzed the mixture of MegaMix A and MegaMix B in different temperatureprogramed modes, the same compound eluted at different column temperatures. In general, the elution temperature of compounds in either MegaMix A or MegaMix B was increased with the increase of temperature ramp rate (data not shown). The low elution temperature was corresponding to ramp rate $4{ }^{\circ} \mathrm{C} / \mathrm{min}$ and the high elution temperature was corresponding to ramp rate $6{ }^{\circ} \mathrm{C} / \mathrm{min}$. Therefore, the temperature range was calculated by subtracting the elution temperature of ramp rate $4{ }^{\circ} \mathrm{C} / \mathrm{min}$ from the elution temperature of ramp rate $6{ }^{\circ} \mathrm{C} / \mathrm{min}$. The average range of the elution temperature for compounds in MegaMix A is $15.2{ }^{\circ} \mathrm{C}$ and $16.2{ }^{\circ} \mathrm{C}$ for compounds in MegaMix B. 
Table 2.8. The second dimension retention indices of compounds identified from MegaMix A by surface fitting.

\begin{tabular}{|c|c|c|c|c|c|c|c|c|c|c|}
\hline \multirow{2}{*}{$\begin{array}{l}\text { Chromatographic conditions } \\
\text { Secondary oven offset }\left({ }^{\circ} \mathrm{C}\right)\end{array}$} & \multicolumn{8}{|c|}{$\begin{array}{l}\text { Retention index of MegaMix A in different } \\
\text { chromatographic conditions }\end{array}$} & \multirow[t]{4}{*}{$\begin{array}{l}R S D \text { of } \\
\operatorname{RI}(\%)^{\mathrm{a}}\end{array}$} & \multirow[t]{4}{*}{$\begin{array}{l}{ }^{2} T_{e} \\
\text { range } \\
\left({ }^{\circ} \mathrm{C}\right)^{\mathrm{b}}\end{array}$} \\
\hline & +5 & +5 & +5 & +5 & +5 & +5 & +5 & +10 & & \\
\hline Ramp rate $\left({ }^{\circ} \mathrm{C} / \mathrm{min}\right)$ & 4 & 4.5 & 5 & 5 & 5.5 & 5.5 & 6 & 5 & & \\
\hline Modulation period (s) & 5 & 5 & 5 & 4 & 5 & 4 & 5 & 4 & & \\
\hline N-Nitrosodimethylamine & 974 & 975 & 976 & 979 & 978 & 978 & 978 & 979 & 0.19 & 9.5 \\
\hline Aniline & 1233 & 1240 & 1245 & 1251 & 1250 & 1250 & 1255 & 1253 & 0.57 & 13.9 \\
\hline Phenol & 1189 & 1193 & 1197 & 1201 & 1201 & 1200 & 1203 & 1204 & 0.40 & 13.4 \\
\hline Bis(2-chloroethyl) ether & 1205 & 1208 & 1212 & 1214 & 1215 & 1215 & 1217 & 1217 & 0.34 & 13.4 \\
\hline 2-Chlorophenol & 1199 & 1205 & 1209 & 1217 & 1216 & 1213 & 1216 & 1218 & 0.50 & 14.0 \\
\hline Benzyl alcohol & 1278 & 1285 & 1290 & 1299 & 1297 & 1296 & 1299 & 1300 & 0.56 & 14.4 \\
\hline 2,2'-Oxybis(2-chloropropane) & 1234 & 1237 & 1239 & 1244 & 1243 & 1240 & 1244 & 1244 & 0.28 & 14.5 \\
\hline 2-Methylphenol & 1274 & 1278 & 1282 & 1289 & 1288 & 1286 & 1290 & 1291 & 0.45 & 14.5 \\
\hline $\mathrm{N}$-nitrosodipropylamine & 1288 & 1291 & 1296 & 1302 & 1300 & 1299 & 1302 & 1303 & 0.41 & 14.7 \\
\hline 3-Methylphenol & 1295 & 1301 & 1304 & 1313 & 1310 & 1308 & 1311 & 1313 & 0.47 & 14.2 \\
\hline Hexachloroethane & 1261 & 1266 & 1271 & 1275 & 1273 & 1273 & 1278 & 1275 & 0.40 & 15.0 \\
\hline Isophorone & 1355 & 1362 & 1366 & 1374 & 1371 & 1371 & 1374 & 1375 & 0.49 & 14.7 \\
\hline 2-Nitrophenol & 1396 & 1404 & 1411 & 1422 & 1419 & 1419 & 1425 & 1422 & 0.68 & 15.0 \\
\hline 2,4-Dimethylphenol & 1378 & 1382 & 1387 & 1396 & 1394 & 1393 & 1396 & 1398 & 0.50 & 15.0 \\
\hline Bis(2-chloroethoxy)-Methane & 1403 & 1409 & 1414 & 1422 & 1421 & 1420 & 1422 & 1424 & 0.48 & 14.7 \\
\hline 2,4-DichloroPhenol & 1413 & 1420 & 1425 & 1435 & 1434 & 1434 & 1437 & 1438 & 0.60 & 14.2 \\
\hline 4-Chloroaniline & 1511 & 1522 & 1530 & 1542 & 1540 & 1540 & 1547 & 1544 & 0.76 & 15.2 \\
\hline 4-Chloro-3-methylphenol & 1562 & 1571 & 1579 & 1588 & 1587 & 1586 & 1592 & 1591 & 0.62 & 15.7 \\
\hline 2-Methylnaphthalene & 1580 & 1591 & 1600 & 1611 & 1609 & 1608 & 1615 & 1614 & 0.71 & 15.9 \\
\hline
\end{tabular}


1-Methylnaphthalene

1,3-Cyclopentadiene, 1,2,3,4,5,5-

hexachloro-

2,4,6-Trichlorophenol

2,4,5-Trichlorophenol

2-Chloronaphthalene

2-Nitroaniline

1,4-Dinitrobenzene

1,3-Dinitrobenzene

Dimethyl phthalate

2,6-Dinitrotoluene

1,2-Dinitrobenzene

Acenapthylene

3-Nitroaniline

Acenaphthene

2,4-dinitro-phenol

Dibenzofuran

2,4-Dinitrotoluene

2,3,5,6-Tetrachlorophenol

2,3,4,6-Tetrachlorophenol

Diethyl Phthalate

Fluorene

4-Chlorophenyl phenyl ether

4-Nitroaniline

4,6-Dinitro-2-methylphenol

Diphenylamine

Azobenzene

4-Bromophenyl phenyl ether

Hexachlorobenzene

$\begin{array}{llllllllll}1612 & 1623 & 1635 & 1644 & 1644 & 1643 & 1649 & 1648 & 0.75 & 16.4 \\ 1548 & 1557 & 1563 & 1573 & 1569 & 1568 & 1571 & 1574 & 0.54 & 16.2 \\ 1637 & 1648 & 1655 & 1666 & 1663 & 1665 & 1672 & 1677 & 0.73 & 16.0 \\ 1645 & 1655 & 1663 & 1675 & 1674 & 1672 & 1679 & 1678 & 0.68 & 16.2 \\ 1696 & 1707 & 1718 & 1729 & 1729 & 1729 & 1735 & 1733 & 0.75 & 16.4 \\ 1809 & 1819 & 1830 & 1838 & 1842 & 1841 & 1851 & 1844 & 0.72 & 16.0 \\ 1852 & 1859 & 1869 & 1877 & 1882 & 1880 & 1889 & 1883 & 0.63 & 15.7 \\ 1863 & 1871 & 1881 & 1889 & 1893 & 1892 & 1901 & 1893 & 0.64 & 16.0 \\ 1817 & 1826 & 1836 & 1844 & 1849 & 1846 & 1852 & 1850 & 0.64 & 15.9 \\ 1860 & 1868 & 1877 & 1885 & 1891 & 1890 & 1898 & 1892 & 0.66 & 15.9 \\ 1908 & 1915 & 1924 & 1932 & 1937 & 1937 & 1946 & 1938 & 0.63 & 16.4 \\ 1818 & 1831 & 1844 & 1854 & 1858 & 1857 & 1866 & 1861 & 0.83 & 16.5 \\ 1927 & 1935 & 1945 & 1954 & 1960 & 1959 & 1969 & 1962 & 0.69 & 16.5 \\ 1851 & 1864 & 1876 & 1885 & 1890 & 1889 & 1896 & 1893 & 0.79 & 16.7 \\ 1916 & 1926 & 1938 & 1944 & 1951 & 1951 & 1957 & 1952 & 0.70 & 15.9 \\ 1881 & 1894 & 1905 & 1916 & 1920 & 1920 & 1927 & 1923 & 0.79 & 16.4 \\ 1939 & 1948 & 1960 & 1968 & 1974 & 1971 & 1980 & 1975 & 0.68 & 16.2 \\ 1889 & 1902 & 1913 & 1923 & 1925 & 1926 & 1929 & 1930 & 0.72 & 16.2 \\ 1904 & 1916 & 1927 & 1937 & 1940 & 1940 & 1945 & 1949 & 0.75 & 16.2 \\ 1954 & 1965 & 1974 & 1983 & 1985 & 1985 & 1988 & 1989 & 0.59 & 15.9 \\ 1971 & 1986 & 2000 & 2009 & 2013 & 2014 & 2020 & 2018 & 0.80 & 16.4 \\ 1947 & 1959 & 1970 & 1980 & 1983 & 1984 & 1988 & 1987 & 0.70 & 16.2 \\ 2118 & 2126 & 2135 & 2142 & 2152 & 2152 & 2159 & 2150 & 0.63 & 16.2 \\ 2021 & 2030 & 2041 & 2051 & 2055 & 2056 & 2064 & 2058 & 0.69 & 16.5 \\ 2031 & 2043 & 2055 & 2063 & 2071 & 2068 & 2077 & 2071 & 0.71 & 16.2 \\ 2001 & 2015 & 2026 & 2035 & 2041 & 2039 & 2044 & 2042 & 0.71 & 16.5 \\ 2088 & 2100 & 2112 & 2122 & 2127 & 2126 & 2132 & 2129 & 0.69 & 16.9 \\ 2074 & 2089 & 2100 & 2112 & 2115 & 2116 & 2121 & 2120 & 0.75 & 16.7\end{array}$




\begin{tabular}{|lllllllllll|} 
Pentachloro-phenol & 2166 & 2179 & 2193 & 2202 & 2209 & 2209 & 2218 & 2213 & 0.77 & 16.9 \\
Anthracene & 2261 & 2276 & 2290 & 2300 & 2309 & 2307 & 2319 & 2310 & 0.80 & 17.0 \\
Phenanthrene & 2271 & 2286 & 2299 & 2309 & 2317 & 2317 & 2329 & 2319 & 0.79 & 17.2 \\
Carbazole & 2380 & 2394 & 2404 & 2413 & 2424 & 2422 & 2433 & 2423 & 0.69 & 16.7 \\
di-n-Butyl phthalate & 2338 & 2348 & 2356 & 2370 & 2367 & 2370 & 2369 & 2373 & 0.50 & 16.0 \\
Fluoranthene & 2638 & 2652 & 2668 & 2678 & 2690 & 2690 & 2701 & 2688 & 0.76 & 17.5 \\
Pyrene & 2735 & 2749 & 2765 & 2774 & 2786 & 2784 & 2800 & 2785 & 0.73 & 17.7 \\
Benzyl butyl phthalate & 2892 & 2904 & 2918 & 2931 & 2940 & 2936 & 2943 & 2937 & 0.59 & 16.5 \\
Bis(2-ethylhexyl) adipate & 2608 & 2613 & 2615 & 2643 & 2629 & 2627 & 2617 & 2636 & 0.44 & 15.7 \\
Benz[a]anthracene & 3157 & 3172 & 3187 & 3201 & 3201 & 3208 & 3157 & 3194 & 0.59 & 13.0 \\
Chrysene & 3156 & 3202 & 3219 & 3233 & 3218 & 3204 & 3176 & 3213 & 0.73 & 12.7 \\
Bis(2-ethylhexyl) phthalate & 2894 & 2902 & 2908 & 2931 & 2893 & 2889 & 2849 & 2881 & 0.76 & 9.2 \\
di-n-Octyl phthalate & 3066 & 3058 & 3057 & 3077 & 3068 & 3068 & 3057 & 3076 & 0.24 & 0.0 \\
\hline
\end{tabular}

${ }^{\text {a }}$ Percentage $(\%)$ relative standard deviation

${ }^{\mathrm{b}}$ Temperature range is calculated by subtracting the temperature value corresponding to ramp $4{ }^{\circ} \mathrm{C} / \mathrm{min} \operatorname{minus} \mathrm{ramp} 6{ }^{\circ} \mathrm{C} / \mathrm{min}$.

Table 2.9. The second dimension retention indices of compounds identified from MegaMix B by surface fitting.

\begin{tabular}{|c|c|c|c|c|c|c|c|c|c|c|}
\hline Chromatographic conditions & \multicolumn{8}{|c|}{$\begin{array}{l}\text { Retention index of MegaMix B in different chromatographic } \\
\text { conditions }\end{array}$} & $\begin{array}{l}R S D \text { of } \\
\text { RI }(\%)^{\mathrm{a}}\end{array}$ & \multirow[t]{4}{*}{$\begin{array}{l}{ }^{2} T_{e} \\
\text { range } \\
\left({ }^{\circ} \mathrm{C}\right)^{\mathrm{b}}\end{array}$} \\
\hline Secondary oven offset $\left({ }^{\circ} \mathrm{C}\right)$ & +5 & +5 & +5 & +5 & +5 & +5 & +5 & +10 & & \\
\hline Ramp rate $\left({ }^{\circ} \mathrm{C} / \mathrm{min}\right)$ & 4 & 4.5 & 5 & 5 & 5.5 & 5.5 & 6 & 5 & & \\
\hline Modulation period (s) & 5 & 5 & 5 & 4 & 5 & 4 & 5 & 4 & & \\
\hline cis-1,3-Dichloro-1-Propene, (Z) & 879 & 880 & 893 & 888 & & & & 887 & 0.57 & 11.5 \\
\hline trans-1,3-Dichloro-Propene€ & 917 & 918 & 923 & 927 & 921 & 921 & & 924 & 0.33 & 10.0 \\
\hline
\end{tabular}




\begin{tabular}{|c|c|c|c|c|c|c|c|c|c|c|}
\hline Toluene & 890 & 893 & 904 & 899 & & & & 901 & 0.58 & 10.0 \\
\hline 1,1,2-Trichloroethane & 929 & 930 & 932 & 937 & 934 & 935 & 937 & 933 & 0.32 & 10.2 \\
\hline Ethyl methacrylate & 895 & 898 & 908 & 906 & & & & 906 & 0.57 & 10.0 \\
\hline 1,3-Dichloropropane & 947 & 948 & 953 & 954 & 952 & 952 & 954 & 951 & 0.26 & 10.5 \\
\hline Dibromochloro-Methane & 979 & 979 & 985 & 987 & 984 & 984 & 986 & 984 & 0.29 & 10.4 \\
\hline Tetrachloroethene & 917 & 918 & 924 & 927 & & & & 927 & 0.47 & 10.3 \\
\hline 1,2-Dibromoethane & 992 & 995 & 998 & 1001 & 996 & 998 & 1001 & 996 & 0.28 & 11.2 \\
\hline Chlorobenzene & 1007 & 1008 & 1011 & 1016 & 1011 & 1014 & 1015 & 1012 & 0.29 & 11.9 \\
\hline 1,1,1,2-Tetrachloroethane & 992 & 1001 & 1005 & 1012 & 1007 & 1008 & 1007 & 1007 & 0.58 & 12.0 \\
\hline Ethylbenzene & 997 & 999 & 1003 & 1008 & 1000 & 1001 & 1003 & 1003 & 0.32 & 12.0 \\
\hline p-Xylene & 1005 & 1005 & 1008 & 1014 & 1006 & 1007 & 1014 & 1010 & 0.35 & 12.0 \\
\hline Styrene & 1062 & 1062 & 1071 & 1072 & 1067 & 1067 & 1066 & 1070 & 0.34 & 11.5 \\
\hline 0-Xylene & 1052 & 1045 & 1053 & 1054 & 1050 & 1050 & 1052 & 1048 & 0.26 & 12.0 \\
\hline Bromoform & 1118 & 1123 & 1129 & 1129 & 1126 & 1131 & 1134 & 1129 & 0.41 & 12.3 \\
\hline cis-1,4-Dichloro-2-butene, , (z)- & 1104 & 1106 & 1112 & 1114 & 1111 & 1113 & 1113 & 1112 & 0.30 & 12.4 \\
\hline 1,1,2,2-Tetrachloroethane & 1112 & 1112 & 1118 & 1119 & 1115 & 1116 & 1119 & 1113 & 0.25 & 12.7 \\
\hline 1,2,3-Trichloropropane & 1127 & 1130 & 1134 & 1140 & 1135 & 1133 & 1139 & 1137 & 0.36 & 13.0 \\
\hline Isopropylbenzene(cumene) & 1062 & 1060 & 1069 & 1072 & 1068 & 1067 & 1072 & 1070 & 0.37 & 13.0 \\
\hline trans-1,4-Dichloro-2-butene, (E)- & & & 1147 & 1153 & 1147 & 1147 & 1152 & 1149 & 0.19 & 6.4 \\
\hline Bromobenzene & 1130 & 1133 & 1137 & 1140 & 1138 & 1139 & 1141 & 1137 & 0.30 & 13.2 \\
\hline 2-Chlorotoluene & 1128 & 1132 & 1137 & 1141 & 1136 & 1136 & & 1140 & 0.38 & 12.0 \\
\hline n- Propylbenzene & 1096 & 1100 & 1106 & 1110 & 1104 & 1104 & 1109 & 1109 & 0.42 & 13.0 \\
\hline 4-Chlorotoluene & 1133 & 1134 & 1140 & 1142 & 1139 & 1139 & 1142 & 1142 & 0.30 & 13.4 \\
\hline 1,3,5-Trimethylbenzene & 1112 & 1112 & 1121 & 1123 & 1120 & 1119 & 1118 & 1118 & 0.33 & 13.9 \\
\hline Pentachloroethane & 1162 & 1164 & 1170 & 1172 & 1172 & 1172 & 1173 & 1167 & 0.32 & 13.7 \\
\hline tert-Butylbenzene & 1133 & 1137 & 1145 & 1148 & 1142 & 1142 & 1145 & 1147 & 0.42 & 13.9 \\
\hline 1,2,4-Trimethylbenzene & 1151 & 1154 & 1160 & 1164 & 1158 & 1161 & & 1162 & 0.37 & 12.0 \\
\hline sec-Butylbenzene & 1155 & 1156 & 1161 & 1167 & 1162 & 1163 & 1163 & 1163 & 0.32 & 14.2 \\
\hline p-Cymene(4-isopropyl toluene & 1168 & 1171 & 1177 & 1182 & 1176 & 1176 & 1180 & 1179 & 0.37 & 14.2 \\
\hline
\end{tabular}




\begin{tabular}{|c|c|c|c|c|c|c|c|c|c|c|}
\hline n-Butylbenzene & 1207 & 1208 & 1214 & 1221 & 1215 & 1215 & 1218 & 1219 & 0.40 & 14.5 \\
\hline 1,2-Dibromo-3-Chloropropane & 1343 & 1351 & 1359 & 1364 & 1362 & 1364 & 1368 & 1367 & 0.59 & 15.0 \\
\hline Benzene, 1,2,3-trichloro- & 1470 & 1479 & 1488 & 1494 & 1494 & 1495 & 1502 & 1498 & 0.66 & 15.9 \\
\hline 1,3-Dichlorobenzene* & 1199 & 1204 & 1210 & 1217 & 1211 & 1212 & 1212 & 1215 & 0.45 & 14.0 \\
\hline 1,4-Dichlorobenzene* & 1215 & 1219 & 1222 & 1232 & 1227 & 1228 & 1231 & 1231 & 0.48 & 14.2 \\
\hline 1,2-Dichlorobenzene* & 1250 & 1255 & 1261 & 1267 & 1264 & 1265 & 1267 & 1269 & 0.49 & 14.5 \\
\hline Nitrobenzene* & 1360 & 1368 & 1377 & 1381 & 1380 & 1383 & 1388 & 1384 & 0.62 & 15.2 \\
\hline Benzene, 1,2,4-trichloro-* & 1413 & 1420 & 1426 & 1434 & 1432 & 1432 & 1436 & 1438 & 0.57 & 15.9 \\
\hline Naphthalene* & 1461 & 1470 & 1481 & 1485 & 1487 & 1489 & 1493 & 1491 & 0.70 & 15.7 \\
\hline $\begin{array}{l}\text { 1,3-Butadiene, 1,1,2,3,4,4- } \\
\text { hexachloro-* }\end{array}$ & 1398 & 1402 & 1408 & 1416 & 1412 & 1413 & 1415 & 1418 & 0.47 & 15.2 \\
\hline
\end{tabular}

a Percentage (\%) relative standard deviation;

${ }^{\mathrm{b}}$ Temperature range is calculated by subtracting the temperature value corresponding to ramp $4{ }^{\circ} \mathrm{C} / \mathrm{min} \operatorname{minus} \mathrm{ramp} 6{ }^{\circ} \mathrm{C} / \mathrm{min}$. 


\subsubsection{Comparison with Literature Reported ${ }^{2} I$ Values}

We previously identified 47 compounds from MegaMix A by GC×GC-MS, where a DB-17ms column was used as the secondary column. ${ }^{76}$ The second dimension retention index of each identified compound was then calculated from the isovolatility curves of reference compounds $n$-alkanes. From these 47 compounds, 44 compounds were identified in this study.

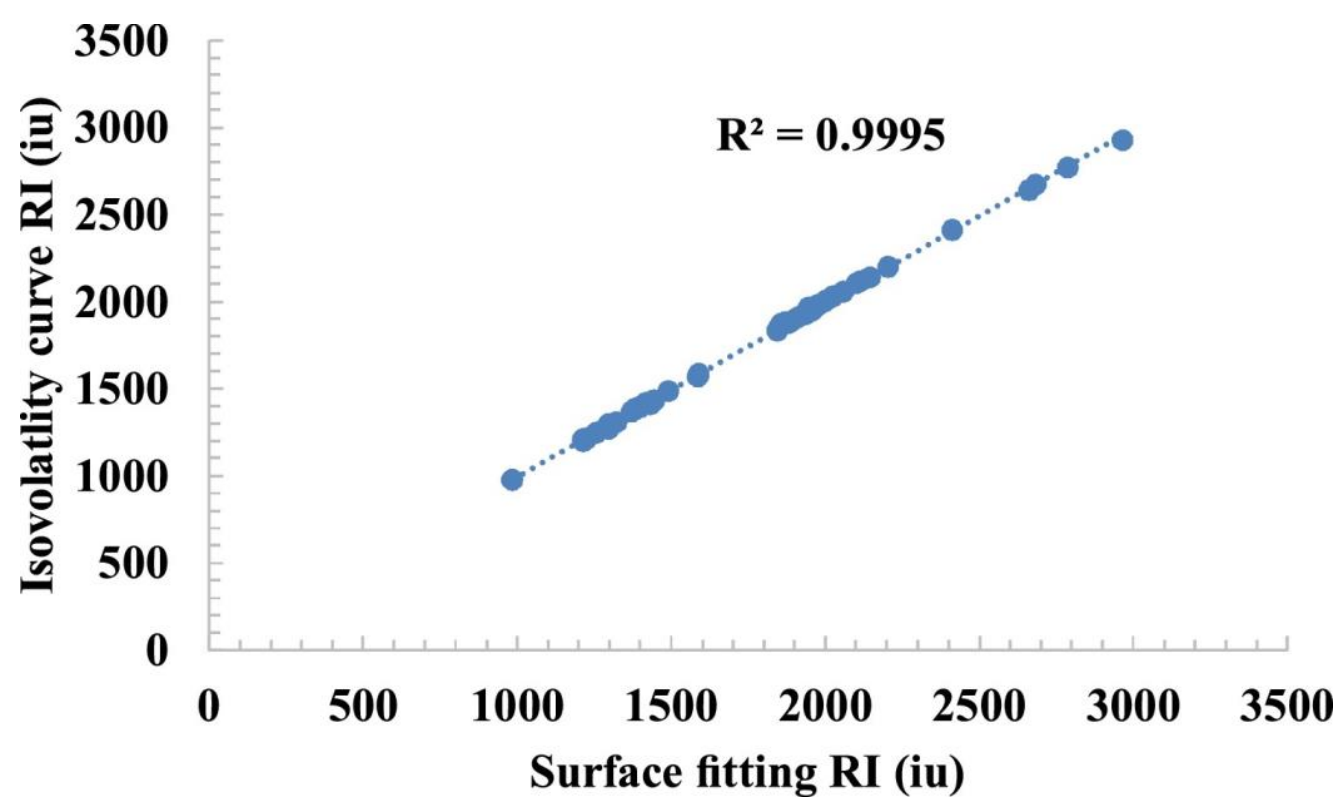

Figure 2.6. Correlation of second dimension retention index of 44 compounds calculated by conventional isovolatility curves and by surface fitting approach.

Figure 2.6 shows the relation of the ${ }^{2} I$ values of the 44 compounds in MegaMix A calculated by surface fitting and by isovolatility curves. The $R^{2}$ value of the fitted curve was 0.9995, indicating a very good agreement between these two methods. 
While the ${ }^{2} I$ values calculated by the conventional isovolatility curves had a good correlation with those calculated by the surface fitting methods, there are some differences between the ${ }^{2} I$ values of individual compounds. Twenty five compounds had the differences in ${ }^{2} I$ values, as calculated by the conventional isovolatility curves and by surface fitting, within $5 \mathrm{iu} ; 8$ compounds within 6-10 iu; 5 compounds within 11-15; and 5 compounds within 16-25 iu; One compound had a variation of 36 iu. Benzyl butyl phthalate had the largest $I$ difference, i.e., 36 iu. The mean ${ }^{2} I$ value of this compound calculated by the isovolatility curve was 2965 iu with standard deviation of \pm 2 iu ${ }^{76}$, while its mean ${ }^{2} I$ value calculated by the surface fitting was 2929 iu with standard deviation of \pm 1 iu.

The standard deviations of the retention index of the 44 compounds calculated from the conventional isovolatility curves are in the range of 1-12 iu with an average standard deviation of 5.2 iu. $^{76}$ However, the standard deviations of the retention index of the 44 compounds calculated by the surface fitting method is in the range of $0-5$ iu with an average standard deviation of $1.6 \mathrm{iu}$. While the true values of the retention index of these compounds remain unknown, the small value of average standard deviation in the calculated retention index using surface fitting method shows that the surface fitting method outperforms the conventional isovolatility curve method by introducing much decreased variation during data analysis.

\subsection{Conclusions}

With the increased use of GCXGC-MS for analysis of complex samples, it is crucial to have a robust method to calculate the second dimension retention index ${ }^{2} I$ for every compound to aid the accuracy of compound identification. A surface fitting method was 
developed to calculate the ${ }^{2} I$ values from the second dimension retention time, ${ }^{2} t_{R}$, and the secondary column temperature, ${ }^{2} T_{e}$. In the surface fitting approach, the second dimension retention time, ${ }^{2} t_{R}$, and second dimension column temperature, ${ }^{2} T_{e}$, formed a $X$ - $Y$ plane and the ${ }^{2} I$ was the $Z$-axis to form the ${ }^{2} I$ surface. The predictability of the surface fitting method was validated using 10-fold cross validation. The average RMSE for the validation set of data was $16.0 \mathrm{iu}$. Analysis of the $\mathrm{GC} \times \mathrm{GC}-\mathrm{MS}$ data acquired from the mixture of MegaMix A and MegaMix B showed that the mean standard deviation of ${ }^{2} I$ values calculated by the surface fitting approach was 1.6 iu for compounds in MegaMix A and 3.4 iu for compounds in MegaMix B. Compared with the literature reported results, there was a very good agreement between the ${ }^{2} I$ values of the compounds in MegaMix A calculated by the surface fitting and by the conventional isovolatility curves with $R^{2}$ value of 0.9995 . However, the small value of standard deviation in the calculated retention index using surface fitting method shows that the surface fitting method outperforms the conventional isovolatility curve method by decresing variation during data analysis.

While the developed method showed excellent reproducibility, the method was not validated by the retention index of these compounds from DB-17ms column. There is no available database containing retention index for DB-17ms column class. Therefore, it is crucial to develop a database for DB-17ms column and validate the developed method. Up until now all the ${ }^{2} I$ calculation method treated the second dimension separation as pseudoisothermal as the second dimension analysis is very fast and column is very short. However, there is actually a temperature change in the second dimension separations though it is small. As second dimension separation was treated as pseudo-isothermal, therefore hold-up time must be calculated. Hold-up calculation methods introduce certain 
degrees of variations which incur certain degree of inaccuracy in ${ }^{2} I$ calculation. It is imperative to develop a second dimension retention index calculation method which will treat the second dimension separation in its actuality and eliminate the erroneous hold-up time calculation step. 
CHAPTER 3

CALCULATING THE RETENTION INDEX OF SECOND DIMENSION SEPARATION IN COMPREHENSIVE TWO-DIMENSIONAL GAS CHROMATOGRAPHY MASS SPECTROMETRY WITHOUT INVOLVING HOLDUP TIME CALCULATION

\subsection{Introduction}

Comprehensive two-dimensional gas chromatography mass spectrometry (GC×GCMS) has become an excellent method for identification and quantification of compounds in complex samples owing to its high separation power. ${ }^{59-61,63,82} \mathrm{GC} \times \mathrm{GC}-\mathrm{MS}$ characterizes each compound by its first dimension retention time $\left({ }^{1} t_{R}\right)$, second dimension retention time $\left({ }^{2} t_{R}\right)$, and mass spectrum. Mass spectrum matching is widely used for compound identification, where a query spectrum is compared with the mass spectra recorded in a database. ${ }^{83,84}$ The top ranked compound whose mass spectrum has the largest spectrum similarity score is usually considered as the compound giving rise to the query spectrum. With the dramatic increase in the number of mass spectra in the database, the chance that the true compound presents in the database is increased. However, the rate of false identification is also increased owing to the increase of the number of similar mass spectra of different compounds. ${ }^{85}$ In order to solve this problem, additional experimental 
information such as retention time has been employed to aid in compound identification. Retention time is usually converted into retention index to reduce its dependency on experimental conditions, and the retention index is then used to filter out the compounds that are identified by mass spectrum matching but have a large retention index difference with that of the experimental value. ${ }^{32,33,86}$

In GC×GC-MS, the two columns are usually operated in temperature gradient mode. The first dimension retention time ${ }^{1} t_{R}$ of a compound $u$ is converted into a linear retention index $I^{T}$ by following the equation $1 .{ }^{28}$ Compounds eluted from the first dimension column during a modulation period $\left(P_{M}\right)$ are subjected to the second dimension column for further separation. Compared with ${ }^{1} t_{R}, P_{M}$ is usually a very small value, e.g., $2-10 \mathrm{~s}$. The temperature change on the second dimension column during one modulation period is small and therefore, the second dimension separation is often considered as being in pseudo-isothermal mode. ${ }^{65-71}$ So ${ }^{2} t_{R}$ of a compound $u$ is converted into Kováts retention index by following the equation 2. ${ }^{29}$

Equation 2 has indirectly been used for ${ }^{2} I$ calculation in GC $\times$ GC-MS because the separation on the second dimension column is achieved within one $P_{M}$ and the bracketing reference compounds may not be found in each $P_{M}$. For this reason, the concept of isovolatility curve mapping was proposed, and each ${ }^{2} I$ value is obtained via interpolation between two adjacent isovolatility curves..$^{40,41,73-76}$ In chapter 2 , we developed a surface fitting approach to calculate ${ }^{2} I$ using $n$-alkanes as references, in which we also approximated the second dimension separation as pseudo-isothermal. ${ }^{87}$ As the second dimension separation is approximated in pseudo-isothermal mode, ${ }^{2} t_{M}$ must be calculated 
in order to convert ${ }^{2} t_{R}$ to ${ }^{2} t_{R}^{\prime}$ for the calculation of ${ }^{2} I$. While several mathematical models have been developed for the calculation of ${ }^{2} t_{M}$, the calculation of ${ }^{2} t_{M}$ always introduces a certain degree of variation. ${ }^{88-92}$ Veenaas and Haglund proposed a PEG- $^{2} I$ system for the calculation of ${ }^{2} I$, where the ${ }^{2} I$ value of each compound was defined based on predefined retention indices of polyethylene glycols (PEGs) with the ${ }^{2} t_{R}$ of $n$-alkanes as the reference (zero) points. ${ }^{93}$ The PEG- $^{2} I$ system is simple and avoided the calculation of ${ }^{2} t_{M}$. However, a new retention index database must be generated for all compounds in order to use the PEG- $^{2} I$ system to identify compounds from complex samples.

The objective of this work was to improve the accuracy of calculating the ${ }^{2} I$ by developing a method that treats the second dimension separation in GC×GC-MS in its actuality, i.e., temperature-programmed mode instead of the approximation approach, i.e., pseudo isothermal mode. By doing so, we can directly calculate the retention index for the second dimension separation, and do not need to approximate the second dimension separation as in pseudo-isothermal mode. This approach eliminates the hold-up time calculation. We constructed a second dimension retention index surface $\left({ }^{2} I_{S}\right)$ using $n$ alkanes as reference compounds, where the unadjusted second dimension retention time ${ }^{2} t_{R}$ and the second dimension column elution temperature ${ }^{2} T_{e}$ formed a $X-Y$ plane and the ${ }^{2} I$ was the $Z$-axis to form ${ }^{2} I_{S}$. The method was then applied to calculate the ${ }^{2} I$ of 387 compounds. The calculated ${ }^{2} I$ of those compounds were compared with their linear retention indices $\left(I^{T}\right)$ calculated from GC-MS.

\subsection{Experimental Section}

\subsubsection{Sample Preparation}


Five hundred and two compounds were used in this study. Six different solutions were prepared by mixing 80 compound standards in different combinations, including a solution containing an odd number of $\mathrm{C}_{5}-\mathrm{C}_{23}$ saturated fatty acid methyl esters (FAMEs) mixed with a solution containing $\mathrm{C}_{4}-\mathrm{C}_{24}$ (even number) carbon saturated FAMEs, a solution containing $\mathrm{C}_{5}-\mathrm{C}_{22}$ saturated fatty acids, a 2-ketone mixture, a n-alcohol mixture, an 1iodoalkane mixture, and an aldehyde mixture. All six mixtures were prepared in dichloromethane with a concentration of $100 \mu \mathrm{g} / \mathrm{mL}$ per compound. Each of MegaMix A and MegaMix B both contain 76 compounds. They were mixed in dichloromethane with a concentration of $50 \mu \mathrm{g} / \mathrm{mL}$ per compound. The six solutions and the mixture of MegaMix A and MegaMix B were analyzed by GC-MS and GC×GC-MS without derivatization.

Another ten solutions were prepared by mixing another 135 compound standards in different solvents including water, ethanol and $\mathrm{HCl}$, depending on the solubility of those compounds. Each of those mixtures was derivatized using $N$-methyl- $N$-(trimethylsilyl) trifluoroacetamide (MSTFA) and $N$-tert-butyldimethylsilyl- $N$-methyltrifluoroacetamide (MTBSTFA), respectively. To derivatize a sample, the sample was first freeze dried and then dissolved in $40 \mu \mathrm{L}$ methoxamine hydrochloride pyridine solution $(20 \mathrm{mg} / \mathrm{mL})$ followed vigorously vortex-mixing for 1 min. Methoxymation was carried out by sonicating for $20 \mathrm{~min}$ and incubating at $60{ }^{\circ} \mathrm{C}$ for $1 \mathrm{~h}$. Derivatization was conducted by adding $40 \mu \mathrm{L}$ of MSTFA or MTBSTFA to the glass vial and incubating at $60{ }^{\circ} \mathrm{C}$ for $1 \mathrm{~h}$. Each stock solution was then transferred to a $\mathrm{GC}$ vial for analysis. The methoxymation and derivatization were conducted prior to the GC-MS and GC×GC-MS analysis.

\subsubsection{GC $\times$ GC-MS Experiments}


The LECO (St. Joseph, MI) Pegasus® 4D GC×GC-MS was configured with a Gerstel MPS2 autosampler and an Agilent 6890 gas chromatograph featuring a LECO two-stage cryogenic modulator and a secondary oven. The first dimension column was a non-polar DB-5ms (phenyl arylene polymer virtually equivalent to a (5\%-phenyl)methylpolysiloxane) $60.0 \mathrm{~m} \times 0.25 \mathrm{~mm}{ }^{l} d_{c} \times 0.25 \mu \mathrm{m}{ }^{l} d_{f}$ column, and the second dimension column was a medium polar BD-17ms ((50\%-phenyl)-methylpolysiloxane) 1.0 $\mathrm{m} \times 0.1 \mathrm{~mm}{ }^{2} d_{c} \times 0.1 \mu \mathrm{m}^{2} d_{f}$ column. Both columns were obtained from Agilent Technologies (Santa Clara, CA), and were connected by means of a press-fit connector before the thermal modulator. The flow rate of ultra-high purity helium carrier gas (99.999\%) was set to $2.0 \mathrm{~mL} / \mathrm{min}$ at a corrected constant flow via pressure ramps with an initial head pressure of $31.9 \mathrm{psi}$. The MS parameters were as follows: MS range $29-800$ $\mathrm{m} / \mathrm{z}$; data acquisition rate $200 \mathrm{spectra} / \mathrm{s}$; temperature of ion source chamber $230{ }^{\circ} \mathrm{C}$; temperature of MS transfer line $280{ }^{\circ} \mathrm{C}$; detector voltage $1641 \mathrm{~V}$; electron energy $70 \mathrm{eV}$. The acceleration voltage was turned on after a solvent delay of $220 \mathrm{~s}$.

A $1 \mu \mathrm{L}$ sample was injected into the inlet chamber of the GC×GC-MS system and the split ratio was set at $10: 1$. The inlet temperature was $280{ }^{\circ} \mathrm{C}$. The thermal modulator was set to $+20{ }^{\circ} \mathrm{C}$ relative to the primary oven. Initial temperature of the first dimension column ${ }^{1} T_{e}$ was set at $60{ }^{\circ} \mathrm{C}$ and kept for $1 \mathrm{~min}$ and then ramped to $285{ }^{\circ} \mathrm{C} .{ }^{l} T_{e}$ was kept at $285{ }^{\circ} \mathrm{C}$ for 13 min to let all compounds elute off the column. Other parameters for analysis of each sample are listed in Table 3.1. 
Table 3.1. GC×GC instrumental parameters

\begin{tabular}{|c|c|c|c|c|}
\hline \multirow[b]{2}{*}{ Sample name } & \multicolumn{3}{|c|}{ Instrument parameters } & \multirow[b]{2}{*}{ Purpose } \\
\hline & Ramp rate $\left({ }^{\circ} \mathrm{C} / \mathrm{min}\right)$ & $\begin{array}{l}P_{M} \\
(\mathrm{~s})\end{array}$ & $\begin{array}{l}\text { Secondary } \\
\text { oven offset } \\
\left(+{ }^{\circ} \mathrm{C} / \mathrm{min}\right)\end{array}$ & \\
\hline n-Alkanes & $\begin{array}{l}0.85,1.00,1.13,1.25 \\
1.50,1.65,1.75,2.00 \\
2.50,3.00,3.50,4.00 \\
4.50,5.00,6.00,7.00\end{array}$ & 4 & 5 & Construction of the ${ }^{2} I_{S}$ \\
\hline $\begin{array}{l}\text { Mixture of } 80 \\
\text { compound } \\
\text { standards }\end{array}$ & $\begin{array}{l}4.00,4.50,5.00,5.50, \\
6.00\end{array}$ & 5 & 5 & Reproducibility evaluation \\
\hline $\begin{array}{l}\text { MTBSTFA } \\
\text { derivatized } \\
\text { compounds }\end{array}$ & 5.00 & $2-5$ & 5 & $\begin{array}{l}\text { Calculation of the }{ }^{2} I \text { and } \\
\text { validation }\end{array}$ \\
\hline $\begin{array}{l}\text { MSTFA } \\
\text { derivatized } \\
\text { compounds }\end{array}$ & 5.00 & $2-5$ & 5 & $\begin{array}{l}\text { Calculation of the }{ }^{2} I \text { and } \\
\text { validation }\end{array}$ \\
\hline $\begin{array}{l}\text { MegaMix A } \\
\& \text { B }\end{array}$ & $\begin{array}{l}3.00,3.50,4.00,4.50 \\
5.00,5.50,6.00\end{array}$ & $4-5$ & $5-10$ & $\begin{array}{l}\text { Robustness of surface } \\
\text { fitting method }\end{array}$ \\
\hline
\end{tabular}

\subsubsection{GC-MS Experiments}

All in-house made mixtures, Megamix A and MegaMix B were respectively analyzed on a Thermo ITQ 1100 Ion Trap MS equipped with a Trace 1310 GC and an AI 1310 auto sampler. The column was a DB-17ms capillary column $\left(45.0 \mathrm{~m} \times 0.1 \mathrm{~mm}^{2} d_{c} \times 0.1 \mu \mathrm{m}^{2} d_{f}\right.$, (50\%-phenyl)-methylpolysiloxane) from Agilent Technologies (Agilent Technologies 
J\&W, Santa Clara, CA, USA). The helium carrier gas (99.999\% purity) flow rate was set to $2.0 \mathrm{~mL} / \mathrm{min}$, and the inlet temperature was $280^{\circ} \mathrm{C}$. The column temperature was programmed with an initial temperature of $60{ }^{\circ} \mathrm{C}$ for $1.0 \mathrm{~min}$ and then ramped at $5{ }^{\circ} \mathrm{C} / \mathrm{min}$ to $290{ }^{\circ} \mathrm{C}$ and maintained at $290{ }^{\circ} \mathrm{C}$ for 13 min. The mass range was set as $29-800 \mathrm{~m} / z$. The ion source chamber was $230{ }^{\circ} \mathrm{C}$ with transfer line temperature of $280{ }^{\circ} \mathrm{C}$.

A mixture of $\mathrm{C}_{7}-\mathrm{C}_{30} n$-alkanes at a concentration of $75 \mu \mathrm{g} / \mathrm{mL}$ was also analyzed by GC-MS to calculate the linear retention indices $\left(I^{T}\right)$ of the compounds in all mixtures.

\subsubsection{Instrument Data Preprocessing}

Table 3.2. Data processing parameters used in LECO ChromaTOF software

\begin{tabular}{ll}
\hline \multicolumn{2}{c}{ Data processing parameters used in LECO ChromaTOF } \\
\hline Baseline Offset & 1 \\
Number of Data Points Averaged for Baseline Smoothing & Auto \\
& GC-MS: 4, GC×GC- \\
Peak Width for Peak Finding & MS: 7.5 \\
Minimum Number of Apexing Masses for Peak Finding & 2 \\
Minimum SNR for Peak Finding & 10 \\
Library Search Mode & Normal, Forward \\
Library Hits Returned per Peak & 10 \\
Minimum/Maximum Molecular Weight Allowed & $29 / 850$ \\
Relative Mass Threshold for Library Searching & 10 \\
Minimum Similarity Match Before Name is Assigned & 600 \\
Libraries Used for Searching & In-House, NIST-14 \\
Mass Used for Area/Height Calculation & Unique \\
Match Required to Combine Peaks in the Second Dimension & 500 \\
Second Dimension Peak Width & 0.15 \\
Minimum SNR for Subpeak to be Retained & 6 \\
Integration Approach & Traditional \\
\hline
\end{tabular}

LECO ChromaTOF software (version 4.51) equipped with the National Institute of Standards and Technology MS database (NIST 2014) was used for instrument control as 
well as for spectrum deconvolution and compound identification. Manufacturer recommended parameters for ChromaTOF were used to reduce the raw instrument data into a peak list of compounds. The true peak spectrum was also exported as part of the information for each chromatographic peak as absolute intensity values. Table 3.2 contains all the parameters for $\mathrm{GC} \times \mathrm{GC}-\mathrm{MS}$ data processing.

GC-MS data were collected by Thermo Xcalibur software (Thermo Fisher Scientific, San Jose, CA, USA) and converted to mzML files using our in-house software, followed by peak picking and compound identification. The retention time of each compound was then converted into $I^{T}$ using $n$-alkanes as reference compounds following Van Den Dool and Kratz equation. ${ }^{28}$

\subsubsection{Construction of the Second Dimension Retention Index Surface}

To construct the second dimension retention index surface, ${ }^{2} I_{S}, \mathrm{C}_{7}-\mathrm{C}_{30} n$-alkanes were analyzed by $\mathrm{GC} \times \mathrm{GC}-\mathrm{MS}$ in temperature-programmed mode at sixteen ramp rates. After chromatographic peak picking and compound identification, ${ }^{2} t_{R}$ and ${ }^{2} T_{e}$ were extracted for each reference compound in each analysis. The ${ }^{2} I$ value of a reference compound with $n$ carbon atoms was defined as $100 \times n$. Therefore, a $n$-alkane was represented by $\left\{{ }^{2} t_{R}\right.$, $\left.{ }^{2} T_{e},{ }^{2} I\right\}$ in each analysis. The ${ }^{2} I_{S}$ was then constructed using the data of all $n$-alkanes, where ${ }^{2} t_{R}$ and ${ }^{2} T_{e}$ formed the $X-Y$ plane and the ${ }^{2} I$ was the $Z$-axis to form the ${ }^{2} I_{S}$. A polynomial surface model in MATLAB (Mathworks, Natick, MA) named 'polyij' was used

to construct the ${ }^{2} I_{S}$, where $i$ was the degree of polynomial in ${ }^{2} t_{R}$, and $j$ was the degree of polynomial in ${ }^{2} T_{e}$. The maximum for both $i$ and $j$ was five in MATLAB.

\subsubsection{Selection of Surface Fitting Model}


To avoid the overfitting in construction of the ${ }^{2} I_{S}, k$-fold cross-validation (CV) was used to select the optimal polynomial surface fitting model. $K$-fold $\mathrm{CV}$ was done by first randomly partitioning the dataset into $k$ subsets of roughly equal size. One subset was used as the testing data to study the performance of the polynomial surface model built by the remaining subsets, i.e., the training data. This process is repeated $k$ times so that each subset was used for testing exactly once. The average cross-validation error was used as a performance indicator of the model. The $k$-fold $\mathrm{CV}$ was then repeated $N$ times, and the model with the smallest estimated error was considered as the optimal model.

After selecting the optimal polynomial model, all dataset was used to construct the second dimension retention index surface ${ }^{2} I_{S}$. The quality of surface fitting was then evaluated based on the distribution of residuals, R-square $\left(R^{2}\right)$, degrees of freedom adjusted R-square $\left(R_{a d j}^{2}\right)$, and root mean squared error $(R M S E)$. Briefly, the distribution of residuals can assess the random errors of surface fitting. $R^{2}$ is the measurement of successfulness of the fitting, which explains the variation of the data. $R_{a d j}^{2}$ adjusts $R^{2}$ based on the residual degrees of freedom ${ }^{87} . R M S E$ is an estimate of the standard deviation of the random component in the data.

\subsection{Results and Discussion}

The six solutions prepared using the 80 compound standards and the mixture of MegaMix A and MegaMix B were not derivatized, while each of the 10 solutions prepared using 135 compound standards was derivatized using MSTFA and MTBSTFA, respectively. All 80 compounds were identified from the six solutions. One hundred and two compounds were identified from the MTBSTFA derivatized ten mixtures while 103 
compounds were identified from the MSTFA derivatized ten mixtures. One hundred and two compounds were identified from the mixture of MegaMix A and MegaMix B. Together, 502 compounds were analyzed in GC×GC-MS and 387 compounds were identified.

\subsubsection{Construction of the Second Dimension Retention Index Surface}

$\mathrm{C}_{7}-\mathrm{C}_{30} n$-alkanes were analyzed in temperature-programmed mode at sixteen ramp rates. After the temperature of the first dimension column reached the final temperature at $285{ }^{\circ} \mathrm{C}$, the column temperature was kept at $285^{\circ} \mathrm{C}$ for 13 min to elute all compounds. Therefore, both the first and the second dimension columns were operated in isothermal mode during the last 13 min. Figure 3.1 shows the elution trend of $\mathrm{C}_{7}-\mathrm{C}_{30} n$-alkanes at different ramp rates, where each series represents the ${ }^{2} t_{R}$ of $n$-alkanes in a chromatographic run at a specific ramp rate.

A total of 346 data points of $n$-alkanes acquired under the temperature-programmed mode were used to construct the ${ }^{2} I_{S}$. To select an optimal polynomial model for surface fitting, $N$ times $(N=100)$ of $k$-fold $(k=10) \mathrm{CV}$ was performed for every combination of $i$ and $j$ in the polynomial surface model 'polyij', where $i=1,2, \ldots, 5$ and $j=1,2, \ldots, 5$. Table 3.3. lists the results of CVs. The optimum model was selected based on minimum

validation RMSE that was defined as the average RMSE of all testing cases for a combination of $i$ and $j$. From these 25 results, the optimal polynomial model was 'poly35', i.e., $i=3$ and $j=5$ with validation $R M S E=4.31$ iu. 


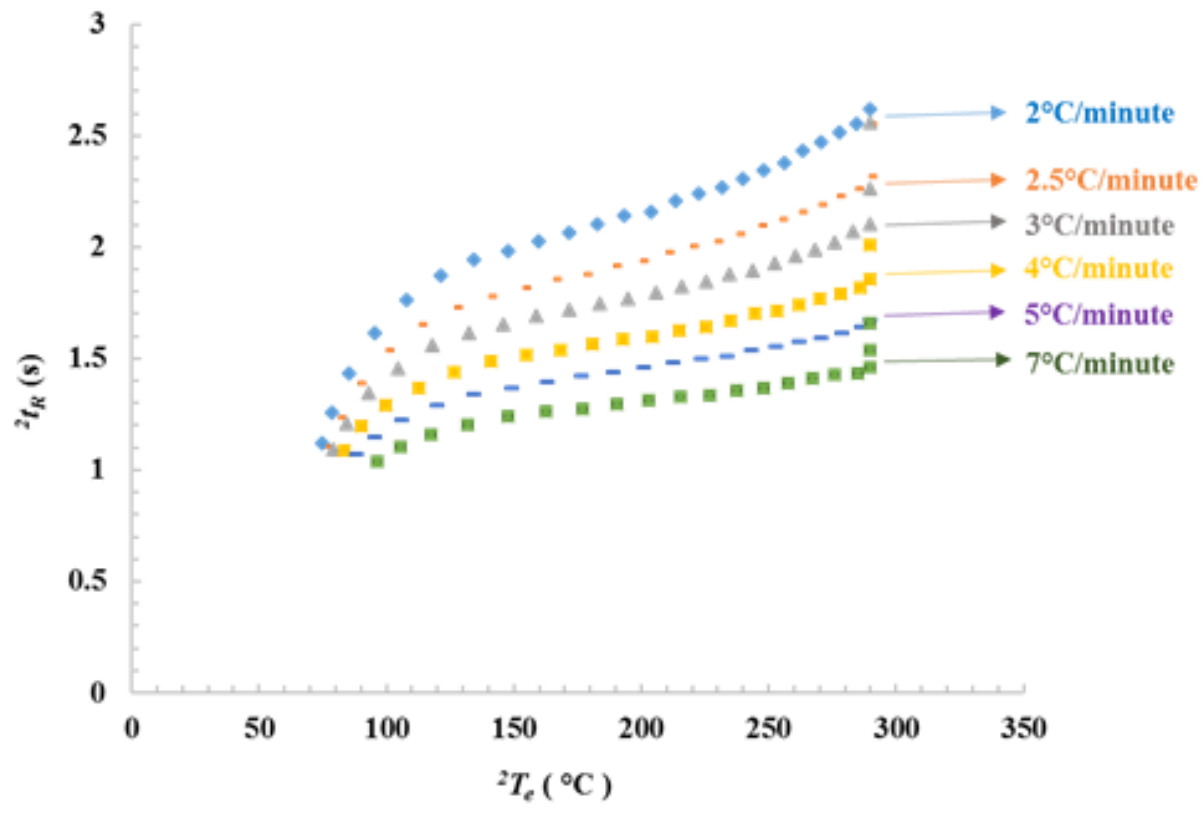

Figure 3.1. Two-dimensional separation space for $n$-alkanes analyzed in temperatureprogrammed mode using different ramp rates. The inflection point in each curve indicates the second dimension column temperature was $285^{\circ} \mathrm{C}$, from which the corresponding $n$ alkane was analyzed in isothermal mode for $13 \mathrm{~min}$.

Table 3.3. Selection of polynomial surface model 'polyij' to construct the second dimension retention index surface using GC×GC-MS data of $n$-alkanes (robust 'LAR')

\begin{tabular}{cccccc}
\hline \multicolumn{2}{c}{ Poly'ij' $^{\prime}$} & $R^{2}$ & & RMSE & $\begin{array}{c}\text { Validation } \\
\text { RMSE }\end{array}$ \\
\cline { 1 - 2 }$i$ & $J$ & & $R_{a d j}^{2}$ & & 94.91 \\
1 & 1 & 0.9787 & 0.9785 & 94.50 & 54.29 \\
1 & 2 & 0.9931 & 0.9930 & 53.93 & 52.25 \\
1 & 3 & 0.9937 & 0.9936 & 51.68 & 50.58 \\
1 & 4 & 0.9942 & 0.9940 & 49.84 & 49.25 \\
2 & 5 & 0.9946 & 0.9944 & 48.35 & 55.35 \\
2 & 1 & 0.9927 & 0.9926 & 55.31 & 20.01 \\
2 & 2 & 0.9999 & 0.9999 & 5.92 & 9.52 \\
2 & 3 & 1.0000 & 1.0000 & 3.97 & 5.98
\end{tabular}




\begin{tabular}{cccccc}
2 & 5 & 1.0000 & 1.0000 & 2.79 & 4.57 \\
3 & 1 & 0.9935 & 0.9933 & 52.71 & 53.54 \\
3 & 2 & 0.9999 & 0.9999 & 7.88 & 18.94 \\
3 & 3 & 1.0000 & 1.0000 & 4.28 & 9.48 \\
3 & 4 & 1.0000 & 1.0000 & 3.46 & 5.89 \\
3 & 5 & 1.0000 & 1.0000 & 2.87 & 4.31 \\
4 & 1 & 0.9937 & 0.9935 & 52.06 & 52.88 \\
4 & 2 & 0.9998 & 0.9998 & 9.67 & 18.26 \\
4 & 3 & 0.9999 & 0.9999 & 4.74 & 8.65 \\
4 & 4 & 1.0000 & 1.0000 & 3.61 & 5.93 \\
4 & 5 & 1.0000 & 1.0000 & 2.96 & 4.33 \\
5 & 1 & 0.9938 & 0.9936 & 51.45 & 52.95 \\
5 & 2 & 0.9997 & 0.9997 & 10.43 & 18.25 \\
5 & 3 & 0.9999 & 0.9999 & 4.94 & 8.25 \\
5 & 4 & 1.0000 & 1.0000 & 3.62 & 5.23 \\
5 & 5 & 1.0000 & 1.0000 & 3.02 & 4.36 \\
\hline
\end{tabular}

After selecting the optimal polynomial model 'poly35', all 346 data points were used to construct the ${ }^{2} I_{S}$. Figure $3.2(\mathrm{~A})$ depicts the ${ }^{2} I_{S}$ constructed from the temperatureprogrammed data of the $n$-alkanes using polynomial surface fitting model 'poly35'. Figure 3.2(B) is the residual plot of the surface fitting. The residuals had a near normal distribution Figure 3.2(C) suggesting that no significant bias was introduced by the polynomial surface model 'poly35', even though a few relatively large residuals showed at the lower end of $X$ $Y$ plane Figure 3.2(B). 
A.

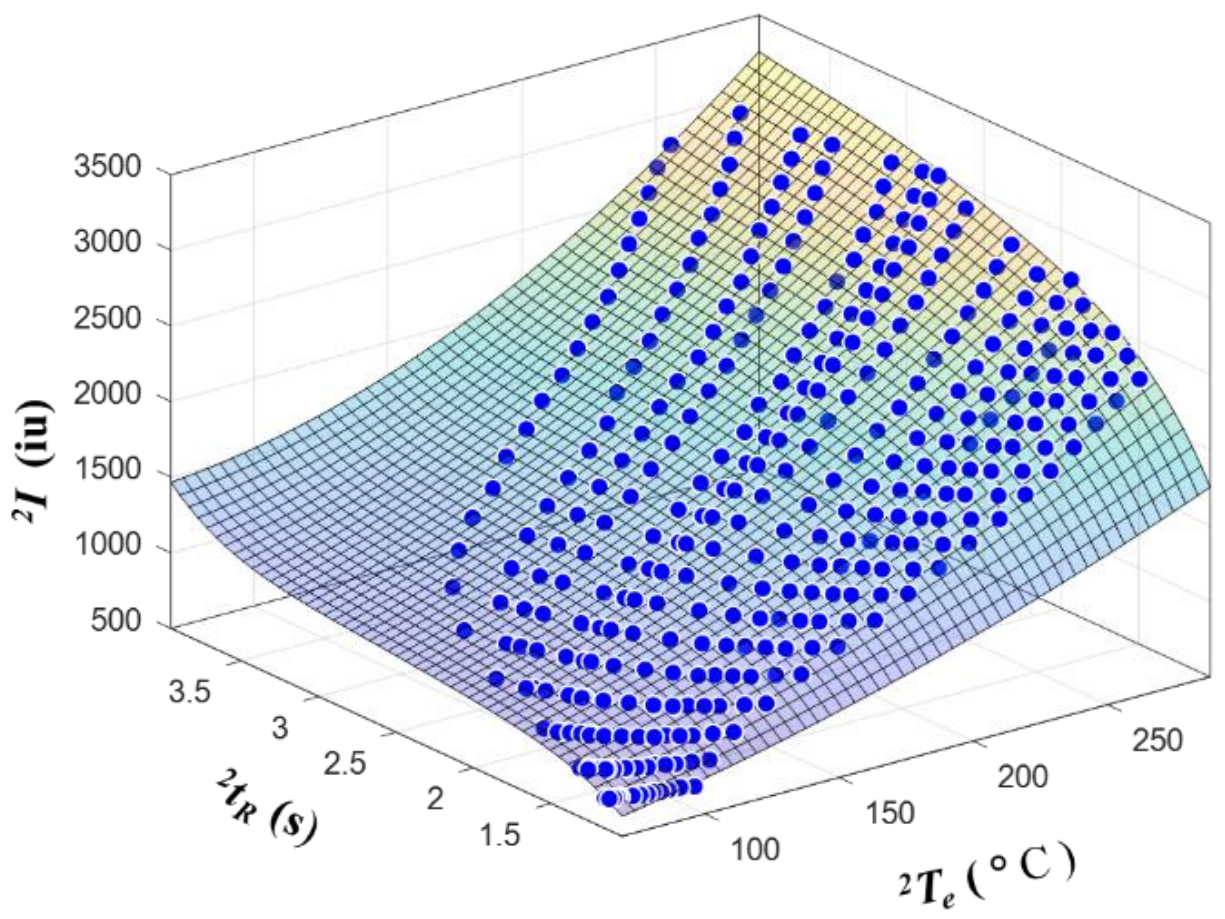

B.

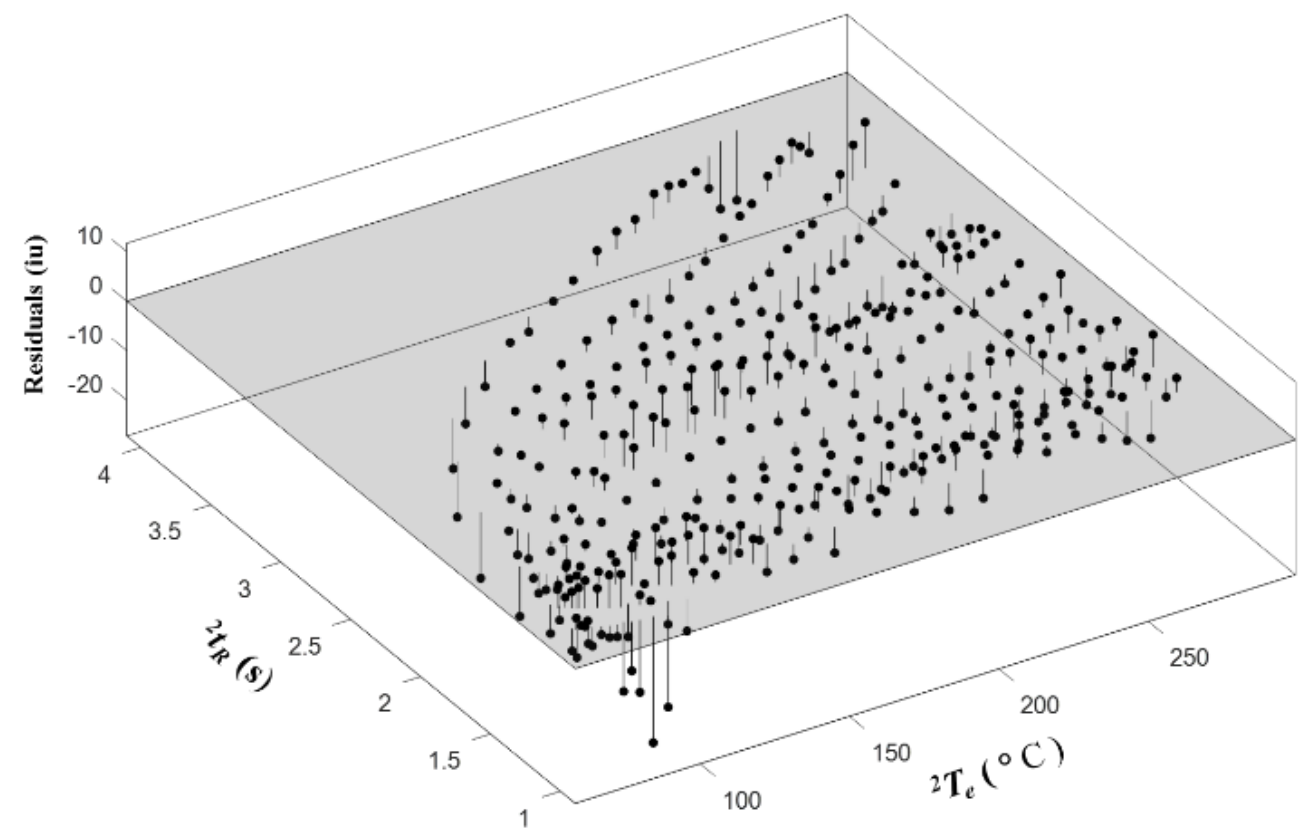


C.

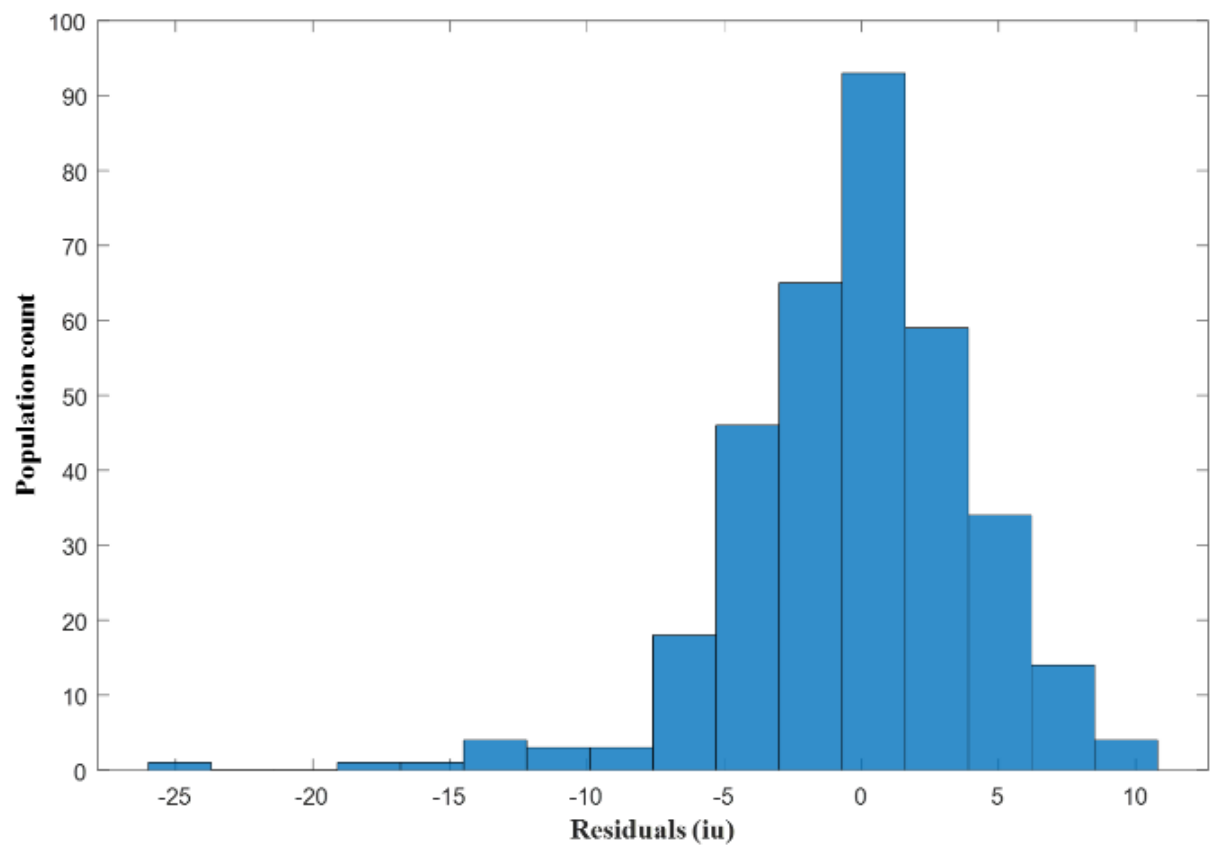

Figure 3.2. The second dimension retention index surface constructed using the GC×GCMS data of $n$-alkanes. The retention index surface is a function of the elution temperature of the second dimension column ${ }^{2} T_{e}$ and the corresponding retention time ${ }^{2} t_{R}$. (A) is the second dimension retention index surface ${ }^{2} I_{S}$. (B) is the residual plot. (C) is the distribution of the residuals.

\subsubsection{Calculation and Validation of the ${ }^{2} I$ of Known Compounds}

For the validation of the developed method, each of the sixteen in-house made mixtures, MegaMix A, and MegaMix B were respectively analyzed by GC×GC-MS and GC-MS in temperature-programed mode using similar chromatographic conditions where ramp rate was $5{ }^{\circ} \mathrm{C} / \mathrm{min}$. The column in GC-MS is the same as the second dimension column in GC $\times$ GC-MS except the column length. Therefore, the ${ }^{2} I$ of a compound 
calculated from the GC $\times$ GC-MS data should be identical to the linear retention index $I^{T}$ of that compound calculated from its GC-MS data with a certain degree of experimental variation.

The ${ }^{2} I$ value of each compound was calculated from the ${ }^{2} I_{S}$ constructed in section 3.1 based on its ${ }^{2} t_{R}$ and ${ }^{2} T_{e}$, while the $I^{T}$ of that compound was calculated from its GCMS data. A total of 387 compounds were identified from the 502 compounds in GC $\times \mathrm{GC}$ MS, where 322 compounds were identified from the 502 compounds in GC-MS. Figure 3.3(A) shows that the second dimension retention indices ${ }^{2} I$ calculated by the surface fitting method and the retention indices $I^{T}$ obtained from the GC-MS data have excellent correlation, with $R^{2}=0.9974$ for 322 compound standards. Figure 3.3(B) depicts the deviation of calculated ${ }^{2} I$ from their corresponding $I^{T}$ of the same compounds, i.e. $\left(I^{T}-\right.$ ${ }^{2} I$, where different color represent the different set of mixture of compound standards. $86 \%$ of the 322 compounds have a retention index difference less than $40 \mathrm{iu}$, while the maximum retention index difference was 79 iu. These results agree with the results from the NIST database. Our lab did a study on NIST database and found that most compounds standard deviation (STD) fall on the range within 40 iu and retention index variations is column's stationary phase dependent ${ }^{94}$.

Compared to the non-polar column, polar columns shows comparatively poor chromatographic reproducibility thus comparatively higher STD of retention index. For our current study, we used DB-17ms column as second dimension column which is a midpolar column. NIST database have not registered any RI value corresponding to the DB$17 \mathrm{~ms}$ column class. 
A.

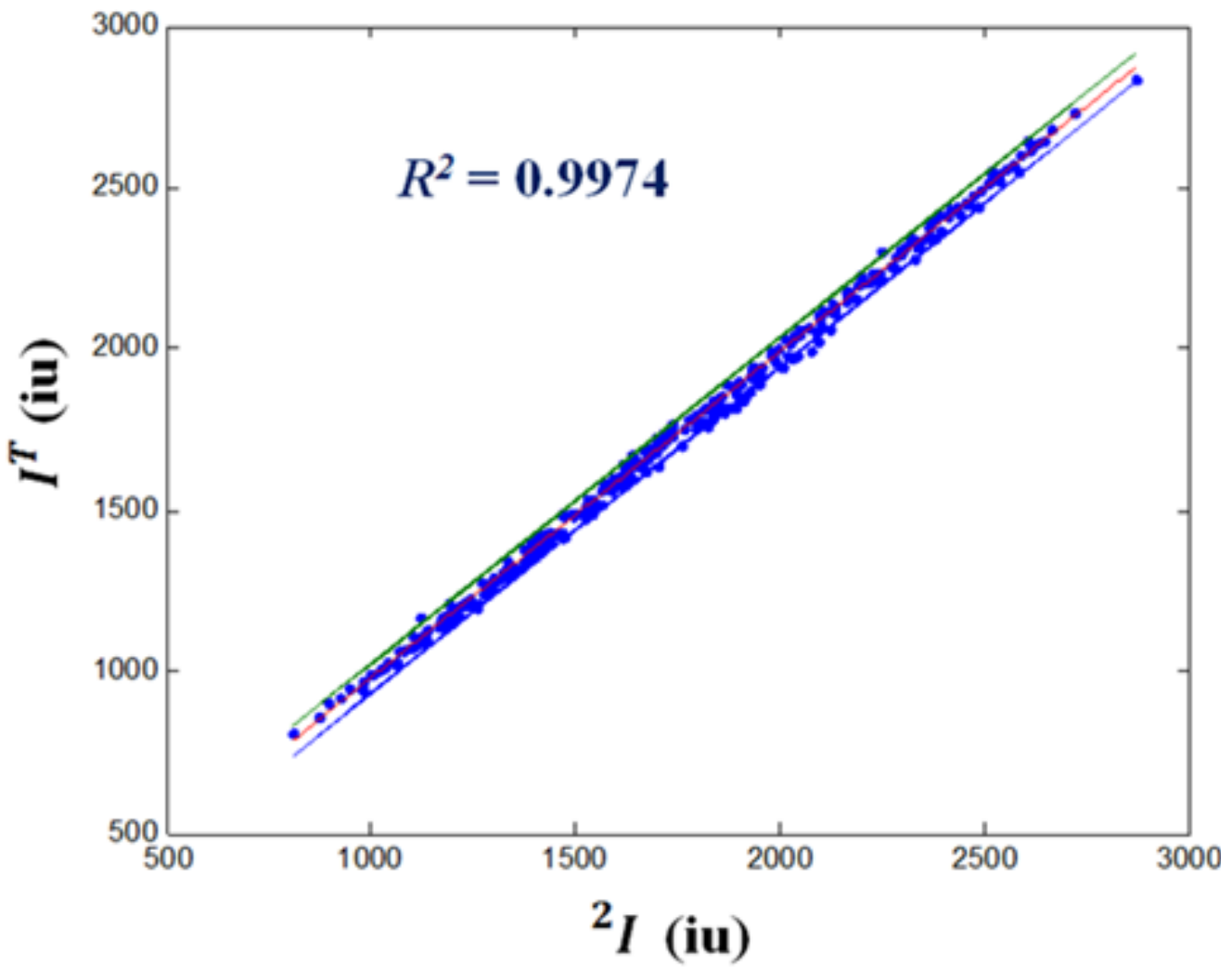


B.

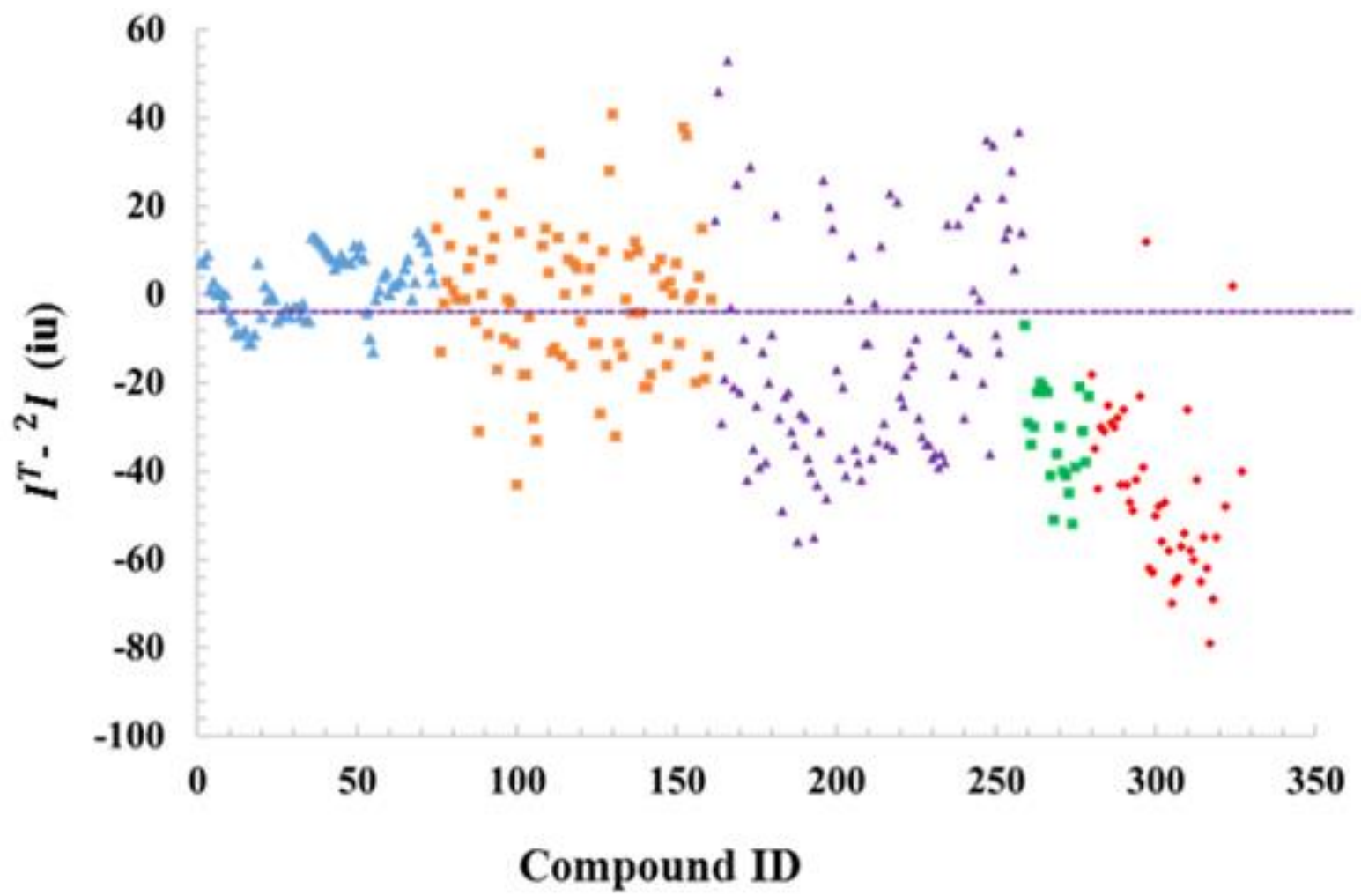


C.

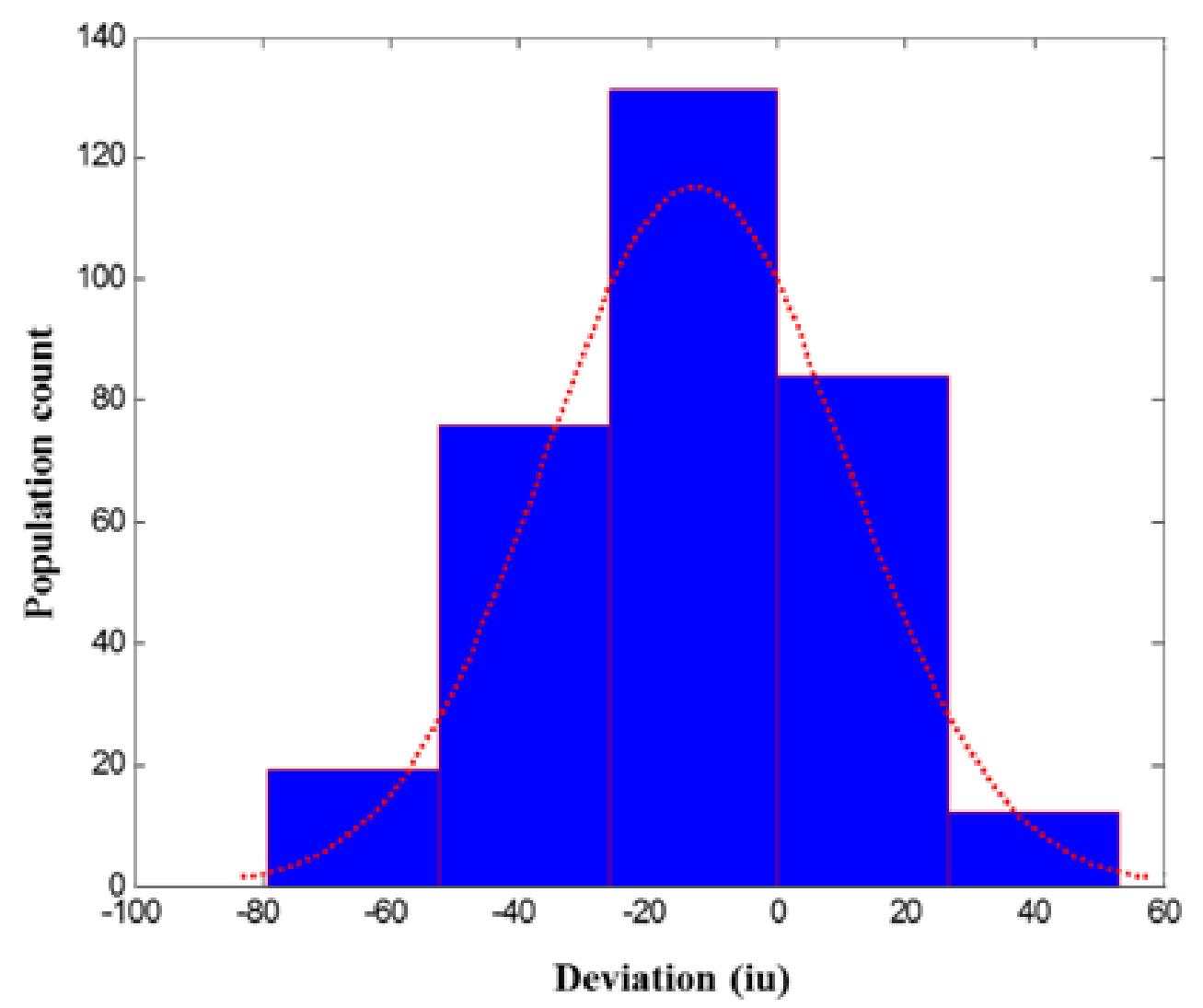

Figure 3.3. Validation of the second dimension retention indices ${ }^{2} I$ calculated using surface fitting method and its corresponding $I^{T}$ calculated from GC-MS data. (A) Correlation between ${ }^{2} I$ of 322 compound standards with their corresponding $I^{T}$. (B) Deviation between $I^{T}$ and ${ }^{2} I$, i.e., $\left|I^{T}-{ }^{2} I\right| .(\Delta)$ represents the mixtures of 80 compound standards, ( $\square$ ) represents the 135 compound standards derivatized with MTBSTFA derivatizing agent, $(\triangle)$ represents the 135 compound standards derivatized with MSTFA derivatizing agent, $(\square)$ represents the MegaMix B compound standards, and $(\diamond)$ represents the MegaMix A compound standards. (C) Distribution of $I^{T}{ }^{2} I$ (iu). 
However, results from the NIST study showed that STD of the retention index increase with the increased polarity, therefore, the STD of the retention index for the DB-17 ms should fall between the non-polar and polar column. Figure 3.3(C) depicts the distribution of the deviation of calculated ${ }^{2} I$ from their corresponding $I^{T}$ which is consistent with the NIST results. ${ }^{94}$

Table 3.4 lists the calculated $I^{T},{ }^{2} I$ and $\left|I^{T}-{ }^{2} I\right|$ of the compound standards detected in the six mixtures that were not derivatized. These six mixtures showed an excellent correlation between the GC $\times$ GC-MS and GC-MS data with an average retention index difference of $5.9 \mathrm{iu}$. The very small difference of retention index between $I^{T}$ and ${ }^{2} I$ can be attributed to the compound structure. These straight chain homologous compounds have similar structure to those reference series, i.e., $n$-alkanes.

Tables 3.5 and 3.6 list the calculated $I^{T},{ }^{2} I$ and $\left|I^{T}-{ }^{2} I\right|$ of the compound standards detected in the ten mixtures that were derivatized by both MSTFA and MTBSTFA. Figure 3.4 shows the effect of derivatization agents on $\left|I^{T}-{ }^{2} I\right|$ of the detected compounds. The MTBSTFA derivatized compounds have small variation in $\left|I^{T}-{ }^{2} I\right|$ with an average variation of $12.5 \mathrm{iu}$, while the MSTFA derivatized compounds have a comparatively large variation with an average of $25.5 \mathrm{iu}$. This was caused by the polarity of the derivatized compounds. In general, the MTBSTFA derivatized compounds are less polar than the MSTFA derivatized compounds owing to the MTBSTFA derivatizing group. With decreasing polarity of the derivatized compounds, the separation mechanism of a compound is similar to the separation mechanism of the non-polar reference series (i.e., $n$-alkanes), thus giving less variation. 
Table 3.4. $I^{T},{ }^{2} I$ and $\left|I^{T}-{ }^{2} I\right|$ of the compound standards detected in the six mixtures of compound standards that were not derivatized

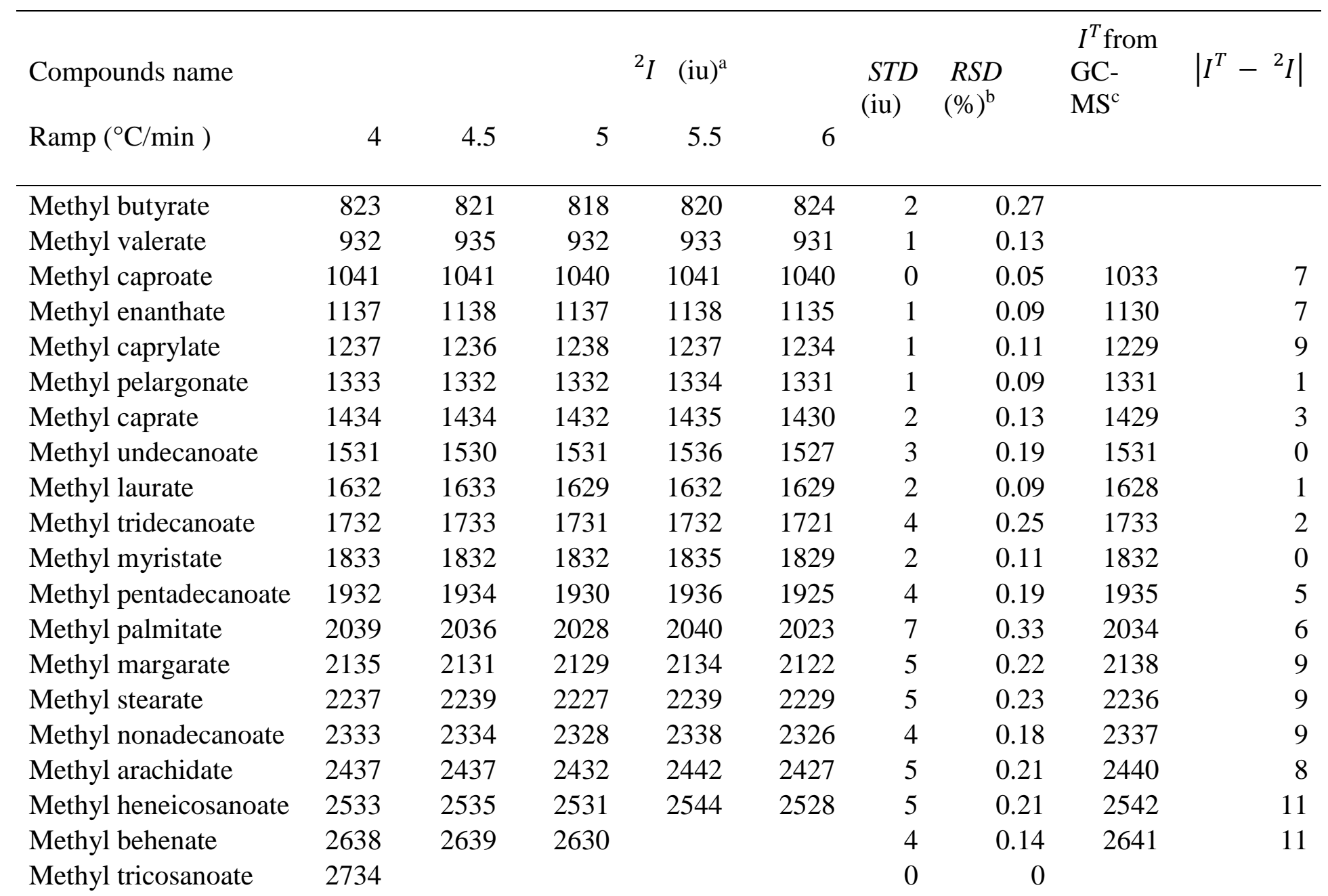




$\begin{array}{lrrrrrrrrr}\text { Butyric acid } & 903 & 912 & 896 & 897 & 900 & 6 & 0.64 & 905 & 9 \\ \text { Valeric acid } & 1005 & 1017 & 1003 & 1004 & 1006 & 5 & 0.53 & 996 & 7 \\ \text { Caproic acid } & 1105 & 1118 & 1103 & 1104 & 1105 & 6 & 0.51 & 1108 & 5 \\ \text { Enanthic acid } & 1200 & 1210 & 1202 & 1203 & 1202 & 3 & 0.28 & 1200 & 2 \\ \text { Caprylic acid } & 1301 & 1312 & 1295 & 1305 & 1301 & 6 & 0.44 & 1296 & 1 \\ \text { Pelargonic acid } & 1403 & 1416 & 1393 & 1406 & 1407 & 7 & 0.53 & 1393 & 0 \\ \text { Capric acid } & 1500 & 1518 & 1491 & 1504 & 1507 & 9 & 0.6 & 1492 & 1 \\ \text { Undecylic acid } & 1599 & 1619 & 1592 & 1611 & 1614 & 10 & 0.62 & 1598 & 6 \\ \text { Lauric acid } & 1700 & 1726 & 1692 & 1708 & 1716 & 12 & 0.7 & 1697 & 5 \\ \text { Tridecylic acid } & 1806 & 1822 & 1793 & 1812 & 1815 & 10 & 0.54 & 1798 & 5 \\ \text { Myristic acid } & 1914 & 1937 & 1898 & 1925 & 1922 & 13 & 0.67 & 1901 & 3 \\ \text { Pentadecylic acid } & 2008 & 2026 & 1994 & 2014 & 2017 & 11 & 0.53 & 1999 & 5 \\ \text { Palmitic acid } & 2112 & 2126 & 2098 & 2122 & 2120 & 10 & 0.47 & 2101 & 3 \\ \text { Margaric acid } & 2213 & 2228 & 2202 & 2227 & 2224 & 10 & 0.46 & 2205 & 3 \\ \text { Stearic acid } & 2318 & 2334 & 2302 & 2323 & 2323 & 10 & 0.45 & 2307 & 5 \\ \text { Nonadecylic acid } & 2420 & 2429 & 2407 & 2422 & 2424 & 7 & 0.3 & 2409 & 2 \\ \text { Arachidic acid } & 2521 & 2528 & 2507 & 2526 & 2527 & 8 & 0.31 & 2513 & 6 \\ \text { Heneicosylic acid } & 2622 & 2636 & 2612 & 2632 & 2613 & 10 & 0.38 & 2618 & 6 \\ \text { Behenic acid } & 2731 & 2733 & & & & 1 & 0.02 & 2726 & \\ \text { 2-Pentanone } & 809 & 810 & 801 & 816 & 795 & 7 & 0.91 & & \\ \text { 2-Hexanone } & 922 & 910 & 917 & 924 & 915 & 5 & 0.55 & & \\ \text { 2-Heptanone } & 1025 & 1024 & 1021 & 1027 & 1023 & 2 & 0.18 & 1008 & 13 \\ \text { 2-Octanone } & 1125 & 1125 & 1122 & 1126 & 1121 & 2 & 0.18 & 1109 & 13 \\ \text { 2-Nonanone } & 1224 & 1227 & 1220 & 1226 & 1222 & 3 & 0.22 & 1208 & 12 \\ \text { 2-Decanone } & 1322 & 1326 & 1319 & 1326 & 1321 & 3 & 0.2 & 1308 & 11 \\ \text { 2-Undecanone } & 1422 & 1427 & 1420 & 1429 & 1419 & 4 & 0.27 & 1410 & 10 \\ \text { 2-Dodecanone } & 1525 & 1527 & 1521 & 1537 & 1521 & 6 & 0.38 & 1512 & 9 \\ \text { 2-Tridecanone } & 1627 & 1626 & 1620 & 1629 & 1620 & 4 & 0.24 & 1612 & 8 \\ \text { 2-Tetradecanone } & 1727 & 1727 & 1720 & 1733 & 1720 & 5 & 0.29 & 1714 & 6 \\ \text { 2-Pentadecanone } & 1833 & 1834 & 1823 & 1831 & 1819 & 6 & 0.32 & 1816 & 7 \\ \text { 2-Hexadecanone } & 1937 & 1937 & 1927 & 1943 & 1923 & 7 & 0.37 & 1918 & 9\end{array}$




\begin{tabular}{lrrrrrrrrr} 
2-Heptadecanone & 2026 & 2025 & 2024 & 2028 & 2021 & 2 & 0.12 & 2017 & 7 \\
2-Octadecanone & 2136 & 2145 & 2130 & 2147 & 2127 & 8 & 0.38 & 2123 & 7 \\
2-Nonadecanoe & 2244 & 2245 & 2232 & 2250 & 2235 & 7 & 0.3 & 2225 & 7 \\
1-Pentanol & 873 & 877 & 873 & 875 & 874 & 2 & 0.18 & 862 & 11 \\
1-Hexanol & 979 & 986 & 981 & 979 & 980 & 3 & 0.26 & 972 & 9 \\
1-Heptanol & 1080 & 1081 & 1080 & 1082 & 1077 & 2 & 0.15 & 1069 & 11 \\
1-Octanol & 1182 & 1185 & 1182 & 1183 & 1180 & 2 & 0.14 & 1174 & 8 \\
1-Nonanol & 1282 & 1286 & 1271 & 1276 & 1271 & 6 & 0.47 & 1275 & 4 \\
1-Undecanol & 1481 & 1480 & 1470 & 1477 & 1469 & 6 & 0.4 & 1480 & 10 \\
1-Dodecanol & 1576 & 1585 & 1569 & 1573 & 1567 & 6 & 0.41 & 1582 & 13 \\
1-Iodobutane & 952 & 956 & 948 & 954 & 954 & 3 & 0.28 & 949 & 1 \\
1-Iodopentane & 1063 & 1065 & 1066 & 1067 & 1069 & 2 & 0.19 & 1065 & 1 \\
1-Iodohexane & 1168 & 1171 & 1172 & 1173 & 1173 & 2 & 0.16 & 1168 & 4 \\
1-Iodoheptane & 1273 & 1278 & 1278 & 1279 & 1279 & 2 & 0.17 & 1273 & 5 \\
1-Iodooctane & 1381 & 1382 & 1380 & 1390 & 1388 & 3 & 0.24 & 1380 & 0 \\
1-Iodononane & 1488 & 1492 & 1490 & 1494 & 1500 & 4 & 0.28 & 1488 & 2 \\
1-Iododecane & 1602 & 1606 & 1598 & 1605 & 1599 & 3 & 0.2 & 1596 & 2 \\
1-Iodoundecane & 1703 & 1708 & 1705 & 1711 & 1709 & 3 & 0.15 & 1702 & 3 \\
1-Iodododecane & 1813 & 1816 & 1813 & 1819 & 1819 & 3 & 0.14 & 1810 & 3 \\
1-Iodohexadecane & 2244 & 2246 & 2243 & 2253 & 2245 & 3 & 0.16 & 2237 & 6 \\
1-Iodooctadecane & 2454 & 2461 & 2457 & 2468 & 2461 & 5 & 0.19 & 2449 & 8 \\
Pentanal & 812 & 809 & 807 & 813 & 808 & 2 & 0.29 & 808 & 1 \\
Hexanal & 923 & 922 & 922 & 923 & 921 & 1 & 0.09 & 919 & 3 \\
Heptanal & 1032 & 1031 & 1030 & 1033 & 1030 & 1 & 0.14 & 1016 & 14 \\
Octanal & 1134 & 1134 & 1131 & 1135 & 1131 & 1 & 0.13 & 1119 & 12 \\
Nonanal & 1233 & 1232 & 1232 & 1233 & 1231 & 1 & 0.07 & 1220 & 12 \\
Decanal & 1332 & 1331 & 1330 & 1333 & 1328 & 2 & 0.14 & 1320 & 10 \\
Undecanal & 1432 & 1428 & 1428 & 1434 & 1427 & 2 & 0.17 & 1422 & 6 \\
Dodecanal & 1534 & 1530 & 1526 & 1531 & 1526 & 3 & 0.19 & 1523 & 3 \\
\hline & & & & & & & & &
\end{tabular}

${ }^{\text {a }}$ Blank cells: means either compound was not identified or it eluted at $290{ }^{\circ} \mathrm{C}$ (isothermal condition in ${ }^{2} T_{e}$ ) 
${ }^{\mathrm{b}}$ Percentage $(\%)$ relative standard deviation

${ }^{\mathrm{c}}$ Linear retention index $I^{T}$ of six standards mixtures that were not derivatized calculated from GC-MS

Table 3.5. $I^{T},{ }^{2} I$ and $\left|I^{T}-{ }^{2} I\right|$ of the compound standards detected in the ten mixtures that were derivatized by MTBSTFA derivatized reagent.

\begin{tabular}{|c|c|c|c|c|}
\hline \multirow[t]{2}{*}{ Compound name } & \multicolumn{4}{|c|}{ MTBSTFA derivatized compounds ${ }^{\mathrm{b}}$} \\
\hline & Derivative $^{\mathrm{a}}$ & ${ }^{2} I$ & $I^{T}$ from GC-MS ${ }^{c}$ & $\left|I^{T}-{ }^{2} I\right|$ \\
\hline \multicolumn{5}{|l|}{ Isobutyric acid } \\
\hline Benzoic acid & TBDMS & 1638 & 1653 & 15 \\
\hline 3-Hydroxybutyric acid & 2TBDMS & 1603 & 1590 & 13 \\
\hline 1-Norleucine & 2TBDMS & 1737 & 1735 & 2 \\
\hline Fumaric acid & 2TBDMS & 1835 & 1838 & 3 \\
\hline Lidocaine & TBDMS & 2107 & 2118 & 11 \\
\hline Adipic acid & 2TBDMS & 2049 & 2050 & 1 \\
\hline $\begin{array}{l}\text { cis-Aconitic acid } \\
\text { Citrulline } \\
\text { Isovaleric acid }\end{array}$ & 3TBDMS & 2453 & 2452 & 1 \\
\hline Nicotinic acid & TBDMS & 1714 & 1737 & 23 \\
\hline Malonic acid & 2TBDMS & 1711 & 1710 & 1 \\
\hline Maleic acid & 2TBDMS & 1854 & 1853 & 1 \\
\hline Sarcosine & 2TBDMS & 1619 & 1625 & 6 \\
\hline 1-Pyroglutamic acid & 2TBDMS & 2106 & 2116 & 10 \\
\hline $\begin{array}{l}\text { 1-Malic acid } \\
\text { Citric acid }\end{array}$ & 3TBDMS & 2123 & 2117 & 6 \\
\hline
\end{tabular}




\begin{tabular}{|c|c|c|c|c|}
\hline Ethylene glycol & 2TBDMS & 1396 & 1365 & 31 \\
\hline Oxalic acid & 2TBDMS & 1625 & 1625 & 0 \\
\hline 3-Aminobutanoic acid & 2TBDMS & 1668 & 1686 & 18 \\
\hline Succinic acid & 2TBDMS & 1834 & 1825 & 9 \\
\hline Glutaric acid & 2TBDMS & 1933 & 1941 & 8 \\
\hline Salicylic acid & 2TBDMS & 2091 & 2104 & 13 \\
\hline Azelaic acid & 2TBDMS & 2362 & 2345 & 17 \\
\hline d-Tartaric acid & 4TBDMS & 2381 & 2404 & 23 \\
\hline \multicolumn{5}{|l|}{ Ferulic acid } \\
\hline Lactic acid & 2TBDMS & 1502 & 1492 & 10 \\
\hline (r)-2-Hydroxybutyric acid & 2TBDMS & 1565 & 1564 & 1 \\
\hline dl-Norleucine & 2TBDMS & 1737 & 1735 & 2 \\
\hline $\begin{array}{l}\gamma \text {-Aminobutyric acid } \\
\alpha \text {-Ketobutyric acid }\end{array}$ & 2TBDMS & 1793 & 1782 & 11 \\
\hline Glycerol & 3TBDMS & 1794 & 1751 & 43 \\
\hline Trans-4-Hydroxy-1-proline & 3TBDMS & 2165 & 2179 & 14 \\
\hline Palmitoleic acid & TBDMS & 2340 & 2322 & 18 \\
\hline \multicolumn{5}{|l|}{ Glutamine } \\
\hline$\beta$-Alanine & 2TBDMS & 1674 & 1656 & 18 \\
\hline 1-Alanine & 2TBDMS & 1540 & 1535 & 5 \\
\hline 1- $\alpha$-Aminoadipic Acid & 3TBDMS & 2394 & 2366 & 28 \\
\hline \multicolumn{5}{|l|}{ D,L- $\beta$-Aminoisobutyric } \\
\hline Acid & 2TBDMS & 1612 & 1644 & 32 \\
\hline 1-Asparagine & 3TBDMS & 2363 & 2374 & 11 \\
\hline 1-Aspartic Acid & 3TBDMS & 2163 & 2178 & 15 \\
\hline \multicolumn{5}{|l|}{ 1-Citrulline } \\
\hline \multicolumn{5}{|l|}{ Cystathionine } \\
\hline \multicolumn{5}{|l|}{ 1-Cystine } \\
\hline 1-Glutamic Acid & 3TBDMS & 2284 & 2289 & 5 \\
\hline Glycine & 2TBDMS & 1588 & 1575 & 13 \\
\hline
\end{tabular}




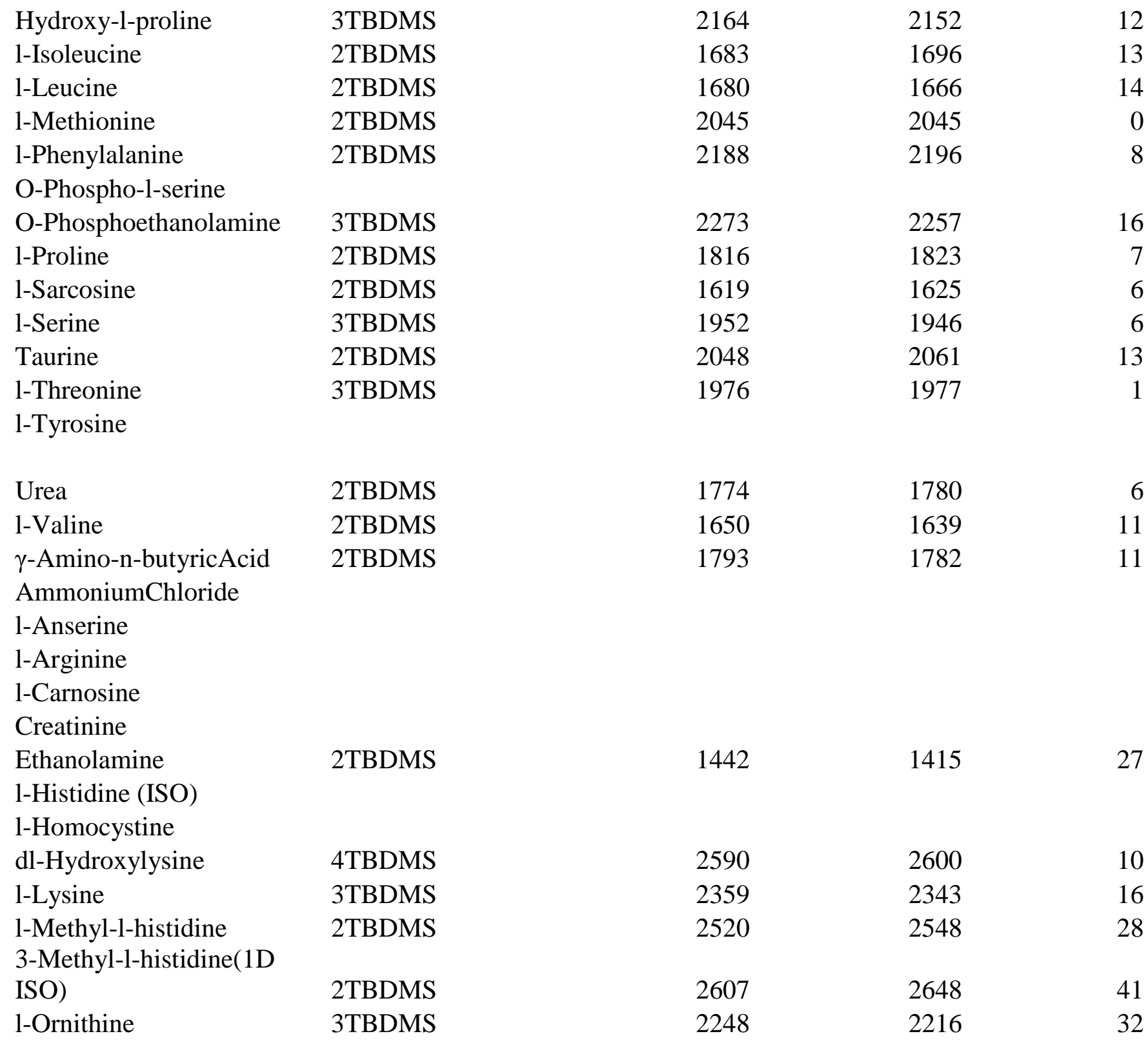


1-Tryptophan (ISO)

Hexanoic acid (C6)

Heptanoic acid (C7)

TBDMS

1427

Octanoic acid (C8)

TBDMS

1416

1516

Nonanoic acid (C9)

TBDMS

1530

1629

Decanoic acid (C10)

TBDMS

1732

Undecanoic acid (C11)

TBDMS

1833

Dodecanoic acid (C12) TBDMS

1935

Tridecanoic acid (C13)

TBDMS

2036

Tetradecanoic acid (C14)

TBDMS

2135

Pentadecanoic acid (C15)

TBDMS

2232

Hexadecanoic acid (C16)

TBDMS

2334

2436

Heptadecanoic acid (C17) TBDMS

2537

1628

1741

1829

1947

2046

2131

2211

2313

2418

Octadecanoic acid (C18)

TBDMS

2543

11

14

1

Nonadecanoic acid (C19)

Eicosanoic acid (C20)

Heneicosanoic acid (C21)

Docosanoic acid (C22)

Tricosanoic acid (C23)

Tetracosanoic acid (C24)

5-Aminovaleric acid

2TBDMS

1897

1887

10

Pipecolic acid

Itaconic acid

2TBDMS

1895

1903

1881

n-Acetyl-1-glutamic acid

2TBDMS

1883

2446

2462

TBDMS

1587

1590

4-Hydroxyphenyllactic

3TBDMS

2648

2648

2520

2329

11

TBDMS

2513
2340

4,methyl 2 oxovaleric acid) 


\begin{tabular}{|c|c|c|c|c|}
\hline Caffeine & $\mathrm{Und}^{\mathrm{c}}$ & 2384 & 2422 & 38 \\
\hline Cotinine & Und & 2195 & 2231 & 36 \\
\hline \multicolumn{5}{|l|}{ Uridine } \\
\hline Uracil & 2TBDMS & 1852 & 1851 & 1 \\
\hline n-Acetylaspartic acid & 3TBDMS & 2489 & 2489 & 0 \\
\hline$\alpha$-Keto-glutarate & 2TBDMS & 2132 & 2112 & 20 \\
\hline Linoelaidic acid & TBDMS & 2554 & 2558 & 4 \\
\hline Pyruvate & TBDMS & 1332 & 1347 & 15 \\
\hline 13-Octadecenoic acid, & TBDMS & 2539 & 2520 & 19 \\
\hline 1-Cysteine & 3TBDMS & 2218 & 2204 & 14 \\
\hline Oleic Acid & TBDMS & 2530 & 2529 & 1 \\
\hline \multicolumn{4}{|l|}{ 1-Homoserine } & \\
\hline \multicolumn{4}{|l|}{ Creatinine } & \\
\hline \multicolumn{4}{|l|}{ 2,3-Butanediol } & \\
\hline \multicolumn{4}{|l|}{ 1-Glutamic acid } & \\
\hline \multicolumn{4}{|l|}{ Niacinamide } & \\
\hline \multicolumn{4}{|l|}{ 1-Lysine } & \\
\hline \multicolumn{4}{|l|}{ n- $\alpha$-Acetyl-1-Lysine } & \\
\hline \multicolumn{4}{|l|}{ Methylmalonic acid } & \\
\hline \multicolumn{4}{|l|}{$\alpha$-Linolenic acid } & \\
\hline \multicolumn{4}{|l|}{ 9-Octadecenoic acid,(E) } & \\
\hline \multicolumn{5}{|c|}{ 9,12-Octadecadienoic acid (Z,Z)- } \\
\hline \multicolumn{5}{|l|}{ 11-Eicosenoic acid, (E)- } \\
\hline \multicolumn{5}{|l|}{ Arachidonic acid } \\
\hline Docosahexaenoic acid & & & & \\
\hline
\end{tabular}

${ }^{a}$ TBDMS: tert-butyl-dimethylsilane

${ }^{\mathrm{b}}$ Blank cells: means either compound was not identified or it eluted at $290{ }^{\circ} \mathrm{C}$ (isothermal condition in ${ }^{2} T_{e}$ )

${ }^{c}$ During derivatization process compound remained underivatized 
Table 3.6. $I^{T},{ }^{2} I$ and $\left|I^{T}-{ }^{2} I\right|$ of the compound standards detected in ten mixtures that were derivatized by MSTFA derivatized reagent

\begin{tabular}{|c|c|c|c|c|}
\hline \multirow[t]{2}{*}{ Compound name } & \multicolumn{4}{|c|}{ MSTFA derivatized compounds ${ }^{\mathrm{b}}$} \\
\hline & Derivative $^{\mathrm{a}}$ & ${ }^{2} I$ & $I^{T}$ from GC-MS & $\left|I^{T}-{ }^{2} I\right|$ \\
\hline \multicolumn{5}{|l|}{ Isobutyric acid } \\
\hline Benzoic acid & TMS & 1391 & 1408 & 17 \\
\hline 3-Hydroxybutyric acid & 2TMS & 1120 & 1166 & 46 \\
\hline 1-Norleucine & $2 \mathrm{TMS}$ & 1336 & 1307 & 29 \\
\hline Fumaric acid & $2 \mathrm{TMS}$ & 1398 & 1379 & 19 \\
\hline Lidocaine & Undc & 2246 & 2299 & 53 \\
\hline Adipic acid & 2TMS & 1600 & 1597 & 3 \\
\hline cis-Aconitic acid & 3TMS & 1833 & 1812 & 21 \\
\hline \multicolumn{5}{|l|}{ Citrulline } \\
\hline \multicolumn{5}{|l|}{ Isovaleric acid } \\
\hline Nicotinic acid & TMS & 1464 & 1489 & 25 \\
\hline Malonic acid & $2 \mathrm{TMS}$ & 1296 & 1274 & 22 \\
\hline Maleic acid & 2TMS & 1430 & 1420 & 10 \\
\hline Sarcosine & 2TMS & 1179 & 1137 & 42 \\
\hline 1-Pyroglutamic acid & 2TMS & 1698 & 1727 & 29 \\
\hline 1-Malic acid & 3TMS & 1534 & 1499 & 35 \\
\hline Citric acid & 4TMS & 1852 & 1827 & 25 \\
\hline
\end{tabular}


Ethylene glycol

Oxalic acid

3-Aminobutanoic acid

Succinic acid

Glutaric acid

Salicylic acid

Azelaic acid

d-Tartaric acid

Ferulic acid

Lactic acid

(r)-2-Hydroxybutyric

acid

dl-Norleucine

$\gamma$-Aminobutyric acid

$\alpha$-Ketobutyric acid

Glycerol

Trans-4-Hydroxy-1-

proline

Palmitoleic acid

Glutamine

$\beta$-Alanine

1-Alanine

1- $\alpha$-Aminoadipic Acid

1- $\alpha$-Amino-n-butyric

Acid

$\mathrm{D}, \mathrm{L}-\beta$-Aminoisobutyric

Acid

1-Asparagine

1-Aspartic Acid

1-Citrulline

Cystathionine
2TMS

2TMS

2TMS

2TMS

2TMS

2TMS

2TMS

4TMS

2TMS

2TMS

2TMS

3TMS

3TMS

3TMS

TMS

3TMS

2TMS

3TMS

2TMS

3TMS

4TMS

3TMS

2TMS
980

1239

1246

1405

1493

1642

1894

1671

1107

1167

1335

1545

1255

1542

2100

1435

1138

1759

1198

1466

1870

1564

2367
941

1226

1208

1385

1484

1660

1866

1622

1084

1145

1304

1511

1199

1515

2072

1398

1098

1704

1155

1435

1896

1518

2387
39

13

38

20

9

18

28

49

23

22

31

34

56

27

28

37

40

55

43

31

26

46

20 
1-Cystine

1-Glutamic Acid

2TMS

1698

1713

1319

1302

1540

1503

1307

1286

1284

1-Leucine

2TMS

1243

1610

1-Methionine

2TMS

1-Phenylalanine

2TMS

1735

1609

1744

15

O-Phospho-l-serine

$\mathrm{O}-$

Phosphoethanolamine

4TMS

1843

1808

1353

1315

1179

1-Sarcosine

2TMS

1337

1137

1326

1767

1756

1387

1350

2070

2068

2016

1372

1983

1383

1232

1-Valine

$\gamma$-Amino-n-butyricAcid

2TMS

3TMS

1203

1511

35

38

42

11

11

37

2

33

11

1545

29

34

AmmoniumChloride

1-Anserine

1-Arginine

1-Carnosine

Creatinine

Ethanolamine

3TMS

1189

1212

23

1-Histidine (ISO)

1-Homocystine

dl-Hydroxylysine

1-Lysine 
1-Methyl-1-histidine

3-Methyl-1-histidine(1D ISO)

1-Ornithine

3TMS

1809

1774

35

1-Tryptophan (ISO)

3TMS

2414

2435

Hexanoic acid (C6)

Heptanoic acid (C7)

TMS

1224

Octanoic acid (C8)

TMS

1318

TMS

1412

TMS

1506

TMS

1602

1698

Dodecanoic acid (C12)

TMS

1795

Tridecanoic acid (C13)

TMS

1893

1295

23

Tetradecanoic acid

TMS

1387

25

1488

1589

1682

16

(C14)

Pentadecanoic acid

(C15)

TMS

1785

10

Hexadecanoic acid

(C16)

TMS

1991

1865

Heptadecanoic acid

(C17)

Octadecanoic acid

(C18)

Nonadecanoic acid

(C19)

Eicosanoic acid (C20)

TMS

2089

1959

Heneicosanoic acid

(C21)

Docosanoic acid (C22)

TMS

2187

2055

TMS

2584

2548

Tricosanoic acid (C23)

Tetracosanoic acid (C24)

5-Aminovaleric acid

Pipecolic acid 
Itaconic acid

n-Acetyl-1-glutamic

acid

Levulinic acid

4-Hydroxyphenyllactic

acid

1-Dihydroorotic acid

Palmitolinoleic acid

4,methyl 2 oxovaleric acid)

Caffeine

Cotinine

Uridine

Uracil

n-Acetylaspartic acid

$\alpha$-Keto-glutarate

Linoelaidic acid

Pyruvate

13-Octadecenoic acid,

1-Cysteine

Oleic Acid

1-Homoserine

Creatinine

2,3-Butanediol

1-Glutamic acid

Niacinamide

1-Lysine

n- $\alpha$-Acetyl-1-Lysine

Methylmalonic acid
2TMS

2TMS

2TMS

1413

1448

1404

1430

9

18

1978

1994

16

3TMS

TMS

TMS

3TMS

2663

2683

20
TMS

3TMS

2TMS

3TMS

2TMS

3TMS

TMS

4TMS

3TMS

2TMS
2297

1389

1434

1593

1640

1664

1733

1845

1905

2013

$\begin{array}{cc}2298 & 1 \\ 1411 & 22 \\ 1433 & 1 \\ 1573 & 20 \\ 1675 & 35 \\ 1628 & 36 \\ 1767 & 34 \\ 1836 & 9 \\ 1892 & 13 \\ 2035 & 22\end{array}$




\begin{tabular}{|c|c|c|c|c|}
\hline$\alpha$-Linolenic acid & TMS & 2297 & 2310 & 13 \\
\hline 9-Octadecenoic acid,(E) & TMS & 2303 & 2318 & 15 \\
\hline 9,12 -Octadecadienoic & & & & \\
\hline $\begin{array}{l}\text { acid }(\mathrm{Z}, \mathrm{Z}) \text { - } \\
\text { 11-Eicosenoic acid, }\end{array}$ & TMS & 2318 & 2346 & 28 \\
\hline$(\mathrm{E})-$ & TMS & 2471 & 2477 & 6 \\
\hline Arachidonic acid & TMS & 2514 & 2551 & 37 \\
\hline Docosahexaenoic acid & TMS & 2719 & 2733 & 14 \\
\hline
\end{tabular}

a TMS: Trimethylsilyl

${ }^{\mathrm{b}}$ Blank cells: means either compound was not identified or it eluted at $290{ }^{\circ} \mathrm{C}$ (isothermal condition in ${ }^{2} T_{e}$ 
A.

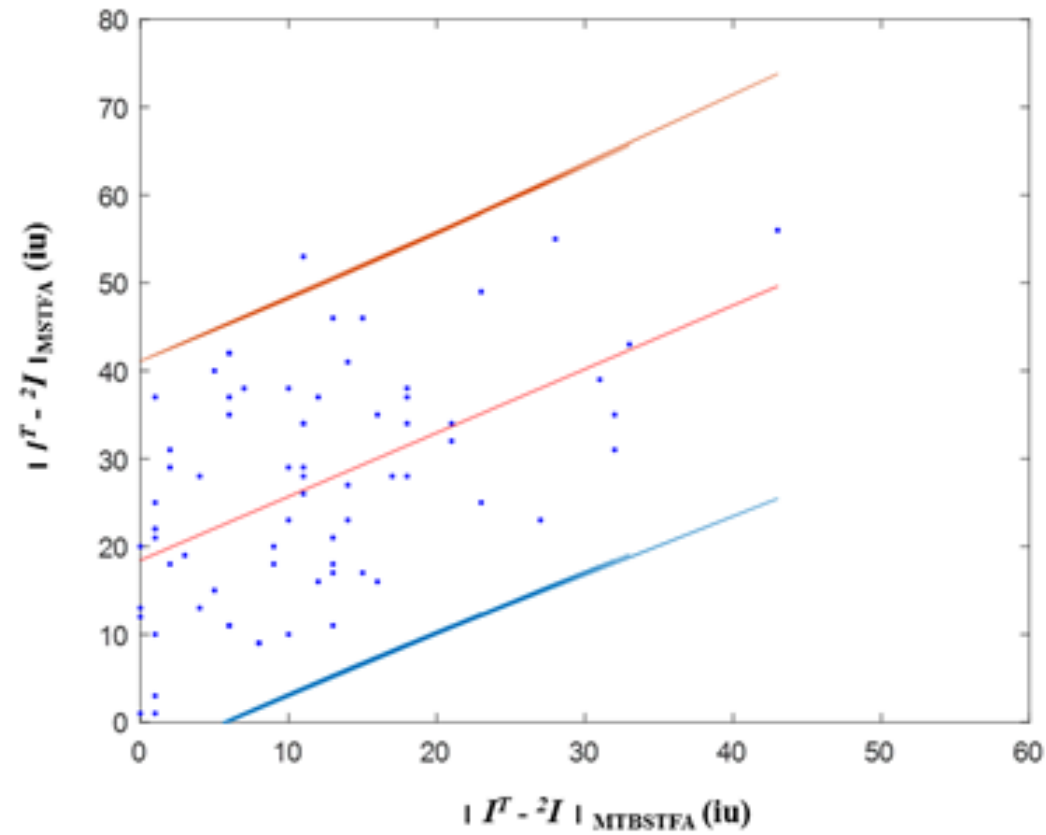

B.

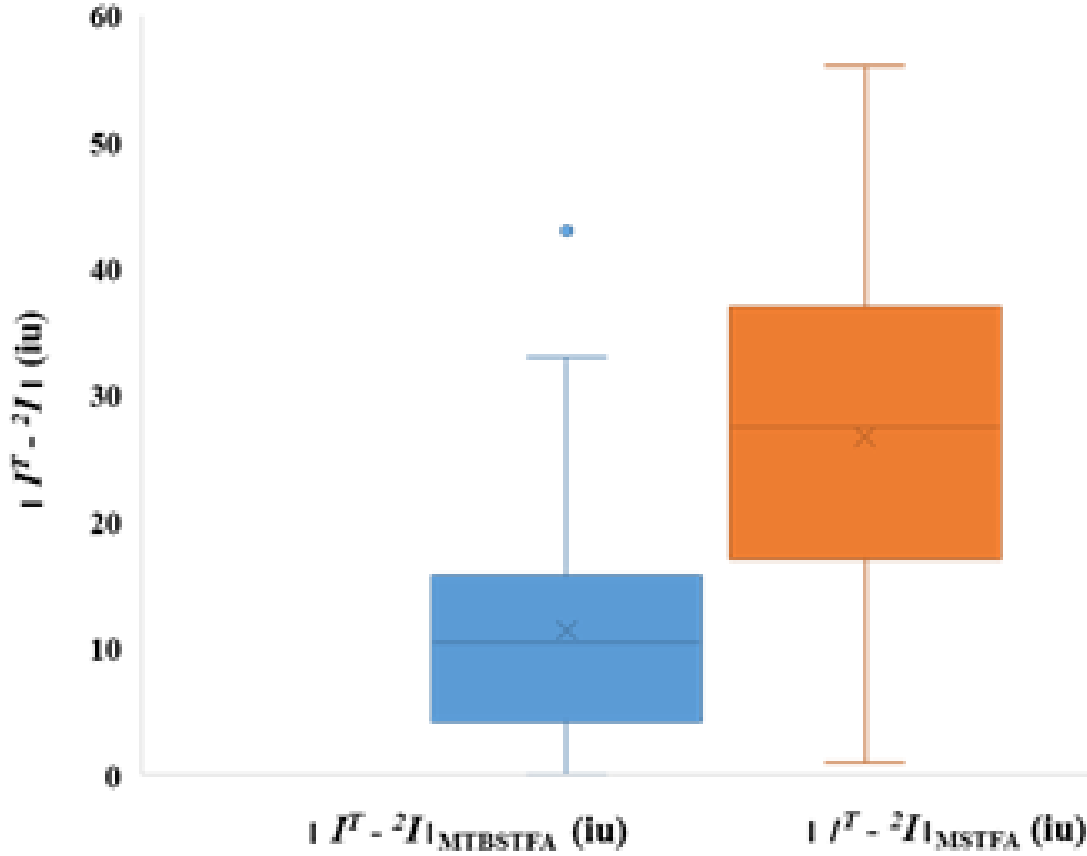


Figure 3.4. Effect of derivatization agent on $\left|I^{T}-{ }^{2} I\right|$. (A) Distribution of $\left|I^{T}-{ }^{2} I\right|$ of MTBSTFA derivatized compounds vis. $\left|I^{T}-{ }^{2} I\right|$ of corresponding compounds derivatized by MSTFA. The red line shows the result of linear fitting while the blue and pink lines are fitting confidence with $95 \%$ confidence interval. (B) Box plot of $\left|I^{T}-{ }^{2} I\right|$ for the MTBSTFA and MSTFA derivatized compounds.

It should be noted that two active hydrogen atoms in $d l$ - $\beta$-aminoisobutyric acid, taurine, and ethanolamine were respectively derivatized by MTBSTFA and three hydrogen atoms were derivatized by MSTFA, which resulted in MSTFA derivatized compounds showing slightly smaller difference between $I^{T}$ and ${ }^{2} I$ than those of the MTBSTFA derivatized compounds. The steric effect hindered the ability of the derivatizing group of MTBSTFA to completely replace the three active hydrogen atoms of those compounds. In contrast, MSTFA derivatizing agent replaced all of the three active hydrogen atoms in each molecule thus making the compound less polar.

MegaMix A and MegaMix B contain compounds with a broad range of cyclization and branching. The ${ }^{2} I$ of these compounds had large deviation from their $I^{T}$ with an average variation of 40.9 iu. Six compounds (i.e., anthracene, phenanthrene, carbazole, fluoranthene, pyrene, benzyl butyl phthalate) had much larger variations because of their structure and were excluded from the average retention index variation calculation. Their structure had polyaromatic ring which are very different from the structure of $n$-alkanes. From the previous study it was found that, polyaromatic hydrocarbon shows poor reproducibility of retention index calculation using $n$-alkanes as reference series. Therefore, a special reference index calculation method was developed exclusively for the 
polyaromatic carbon known as Lee retention index using some polyaromatic compounds as reference series..$^{95}$

Overall, the magnitude of deviation between the calculated ${ }^{2} I$ and the corresponding $I^{T}$ can be attributed to several factors, including compound polarity, structure, and elution temperature, etc. The elution temperature of compounds increases with increased ramp rate as listed in Table 3.7.

Table 3.7. Second dimension elution temperature of 80 standard compounds with different ramp rates

\begin{tabular}{|c|c|c|c|c|c|c|}
\hline \multirow{2}{*}{\begin{tabular}{|l} 
Compounds name \\
Ramp $\left({ }^{\circ} \mathrm{C} / \mathrm{min}\right)$ \\
\end{tabular}} & \multicolumn{5}{|c|}{$I^{2},(\mathrm{iu})^{\mathrm{a}}$} & \multirow[t]{2}{*}{${ }^{2} \mathrm{~T}_{\mathrm{e}} \Delta^{b}$} \\
\hline & 4 & 4.5 & 5 & 5.5 & 6 & \\
\hline \multicolumn{7}{|l|}{ FAME } \\
\hline Methyl butyrate & 84.8 & 86.8 & 89.3 & 91.2 & 93.6 & 8.9 \\
\hline Methyl valerate & 92.1 & 94.7 & 97.6 & 100.0 & 102.6 & 10.5 \\
\hline Methyl caproate & 102.4 & 106.0 & 108.9 & 111.9 & 115.1 & 12.7 \\
\hline Methyl enanthate & 115.8 & 119.5 & 123.0 & 126.1 & 129.6 & 13.9 \\
\hline Methyl caprylate & 129.8 & 133.8 & 137.6 & 141.2 & 144.7 & 14.9 \\
\hline Methyl pelargonate & 144.5 & 148.4 & 152.2 & 155.4 & 159.2 & 14.7 \\
\hline Methyl caprate & 158.1 & 162.3 & 166.0 & 169.6 & 173.2 & 15.0 \\
\hline Methyl undecanoate & 171.5 & 175.4 & 179.3 & 182.9 & 186.7 & 15.2 \\
\hline Methyl laurate & 183.8 & 188.1 & 191.8 & 195.8 & 199.2 & 15.4 \\
\hline Methyl tridecanoate & 195.8 & 199.8 & 203.9 & 207.7 & 211.2 & 15.4 \\
\hline Methyl myristate & 207.1 & 211.4 & 215.1 & 218.7 & 222.7 & 15.5 \\
\hline Methyl pentadecanoate & 217.8 & 221.9 & 226.0 & 229.7 & 233.2 & 15.4 \\
\hline Methyl palmitate & 227.8 & 232.0 & 236.4 & 239.8 & 243.7 & 15.9 \\
\hline Methyl margarate & 237.8 & 242.1 & 246.0 & 249.9 & 253.7 & 15.9 \\
\hline Methyl stearate & 247.1 & 251.1 & 255.1 & 259.0 & 262.7 & 15.5 \\
\hline Methyl nonadecanoate & 255.8 & 260.1 & 264.3 & 268.2 & 271.7 & 15.9 \\
\hline Methyl arachidate & 264.5 & 268.8 & 272.6 & 276.4 & 280.2 & 15.7 \\
\hline Methyl heneicosanoate & 272.8 & 277.0 & 281.0 & 284.7 & 288.7 & 15.9 \\
\hline Methyl behenate* & 280.5 & 284.9 & 288.9 & & & \\
\hline Methyl tricosanoate* & 288.1 & & & & & \\
\hline \multicolumn{7}{|l|}{ Fatty acid } \\
\hline Butyric acid & 88.1 & 91.0 & 93.4 & 95.8 & 98.1 & 10.0 \\
\hline Valeric acid & 97.4 & 100.7 & 103.9 & 106.8 & 109.6 & 12.2 \\
\hline
\end{tabular}




\begin{tabular}{|c|c|c|c|c|c|c|}
\hline Caproic acid & 109.4 & 113.1 & 116.8 & 120.1 & 123.1 & 13.7 \\
\hline Enanthic acid & 123.1 & 127.0 & 131.0 & 134.4 & 137.7 & 14.5 \\
\hline Caprylic acid & 137.8 & 142.0 & 145.6 & 149.0 & 152.7 & 14.9 \\
\hline Pelargonic acid & 150.5 & 154.8 & 158.9 & 161.9 & 165.2 & 14.7 \\
\hline Capric acid & 164.1 & 167.9 & 172.2 & 175.2 & 178.7 & 14.5 \\
\hline Undecylic acid & 176.5 & 180.6 & 184.7 & 188.0 & 191.7 & 15.2 \\
\hline Lauric acid & 188.8 & 192.6 & 196.8 & 200.4 & 203.7 & 14.9 \\
\hline Tridecylic acid & 200.1 & 204.3 & 208.5 & 211.8 & 215.2 & 15.0 \\
\hline Myristic acid & 210.8 & 215.1 & 219.3 & 222.8 & 226.7 & 15.9 \\
\hline Pentadecylic acid & 221.5 & 225.6 & 230.1 & 233.4 & 237.2 & 15.7 \\
\hline Palmitic acid & 231.5 & 235.8 & 240.1 & 243.5 & 247.2 & 15.7 \\
\hline Margaric acid & 241.1 & 245.1 & 249.7 & 253.1 & 256.7 & 15.5 \\
\hline Stearic acid & 250.1 & 254.5 & 258.9 & 262.2 & 266.2 & 16.0 \\
\hline Nonadecylic acid & 259.1 & 263.1 & 267.7 & 271.4 & 274.7 & 15.5 \\
\hline Arachidic acid & 267.8 & 271.8 & 276.4 & 279.7 & 283.7 & 15.9 \\
\hline Heneicosylic acid* & 275.8 & 280.0 & 284.3 & 287.9 & & \\
\hline Behenic acid* & 283.8 & 287.9 & & & & \\
\hline \multicolumn{7}{|l|}{ Ketone } \\
\hline 2-Pentanone & 83.1 & 85.3 & 87.2 & 89.4 & 91.6 & 8.5 \\
\hline 2-Hexanone & 89.1 & 91.7 & 94.3 & 96.8 & 99.1 & 10.0 \\
\hline 2-Heptanone & 98.4 & 101.9 & 104.7 & 107.8 & 110.6 & 12.2 \\
\hline 2-Octanone & 111.1 & 114.6 & 118.1 & 121.5 & 124.7 & 13.5 \\
\hline 2-Nonanone & 125.5 & 129.3 & 133.1 & 136.2 & 139.7 & 14.2 \\
\hline 2-Decanone & 139.8 & 143.9 & 147.6 & 151.3 & 154.7 & 14.9 \\
\hline 2-Undecanone & 154.1 & 158.1 & 162.2 & 165.5 & 169.2 & 15.0 \\
\hline 2-Dodecanone & 167.8 & 172.0 & 175.6 & 179.3 & 183.2 & 15.4 \\
\hline 2-Tridecanone & 180.5 & 184.8 & 188.5 & 192.1 & 196.2 & 15.7 \\
\hline 2-Tetradecanone & 192.8 & 196.8 & 201.0 & 204.5 & 208.2 & 15.4 \\
\hline 2-Pentadecanone & 204.1 & 208.4 & 212.6 & 216.4 & 220.2 & 16.0 \\
\hline 2-Hexadecanone & 215.1 & 219.6 & 223.5 & 227.4 & 231.2 & 16.0 \\
\hline 2-Heptadecanone & 225.8 & 230.1 & 234.3 & 237.9 & 241.7 & 15.9 \\
\hline 2-Octadecanone & 235.8 & 239.9 & 243.9 & 247.6 & 251.7 & 15.9 \\
\hline 2-Nonadecanoe & 245.1 & 249.6 & 253.5 & 257.2 & 261.2 & 16.0 \\
\hline \multicolumn{7}{|l|}{ Alcohol } \\
\hline 1-Pentanol & 87.4 & 89.9 & 92.6 & 94.9 & 97.1 & 9.7 \\
\hline 1-Hexanol & 96.4 & 99.2 & 102.2 & 105.0 & 108.1 & 11.7 \\
\hline 1-Heptanol & 108.8 & 112.0 & 115.5 & 119.2 & 122.1 & 13.4 \\
\hline 1-Octanol & 122.5 & 125.9 & 129.7 & 133.4 & 136.6 & 14.2 \\
\hline 1-Nonanol & 137.1 & 141.6 & 148.9 & 151.8 & 157.1 & 20.0 \\
\hline 1-Undecanol & 168.4 & 171.6 & 178.0 & 180.2 & 186.1 & 17.7 \\
\hline 1-Dodecanol & 186.1 & 185.1 & 192.6 & 196.2 & 200.6 & 14.5 \\
\hline \multicolumn{7}{|l|}{ Iodobutne } \\
\hline 1-Iodobutane & 92.1 & 95.1 & 98.0 & 100.4 & 103.1 & 11.0 \\
\hline 1-Iodopentane & 103.1 & 106.7 & 109.7 & 112.8 & 116.2 & 13.0 \\
\hline 1-Iodohexane & 117.1 & 120.6 & 124.3 & 127.9 & 131.2 & 14.0 \\
\hline
\end{tabular}




\begin{tabular}{|l|r|r|r|r|r|r|}
\hline 1-Iodoheptane & 131.8 & 136.0 & 139.7 & 143.5 & 146.7 & 14.9 \\
\hline 1-Iodooctane & 146.8 & 151.0 & 155.1 & 158.7 & 162.2 & 15.4 \\
\hline 1-Iodononane & 161.5 & 165.6 & 169.7 & 173.3 & 177.2 & 15.7 \\
\hline 1-Iododecane & 175.1 & 179.5 & 183.5 & 187.1 & 191.2 & 16.0 \\
\hline 1-Iodoundecane & 188.1 & 192.3 & 196.4 & 200.4 & 204.2 & 16.0 \\
\hline 1-Iodododecane & 200.5 & 204.6 & 208.9 & 212.7 & 216.2 & 15.7 \\
\hline 1-Iodohexadecane & 243.5 & 247.8 & 251.8 & 255.8 & 259.7 & 16.2 \\
\hline 1-Iodooctadecane & 261.8 & 266.5 & 270.6 & 274.6 & 278.2 & 16.4 \\
\hline Aldehyde & 83.8 & 85.7 & 88.0 & 90.3 & 92.1 & 8.4 \\
\hline Pentanal & 90.1 & 92.9 & 95.5 & 98.1 & 100.6 & 10.5 \\
\hline Hexanal & 100.1 & 103.4 & 106.8 & 109.6 & 112.6 & 12.5 \\
\hline Heptanal & 113.1 & 116.9 & 120.6 & 123.8 & 127.2 & 14.0 \\
\hline Octanal & 127.5 & 131.5 & 135.1 & 138.5 & 142.2 & 14.7 \\
\hline Nonanal & 142.1 & 146.1 & 150.1 & 153.6 & 157.2 & 15.0 \\
\hline Decanal & 156.1 & 160.4 & 164.3 & 167.8 & 171.7 & 15.5 \\
\hline Undecanal & 169.8 & 173.9 & 178.1 & 181.6 & 185.2 & 15.4 \\
\hline Dodecanal &
\end{tabular}

${ }^{\text {a }}$ Blanks means either the compound was not identified or it eluted at $290{ }^{\circ} \mathrm{C}$ (isothermal condition ${ }^{2} T_{e}$ )

${ }^{\mathrm{b}}$ Temperature range is calculated by subtracting the temperature value corresponding to ramp $4{ }^{\circ} \mathrm{C} / \mathrm{min}$ from the $\operatorname{ramp} 6{ }^{\circ} \mathrm{C} / \mathrm{min}$

In general, retention index variations increase with increased elution temperature. However, some compounds did not show this trend, as retention index also depends on the structure . Figure 3.5(A) shows the variations of retention index with increased ramp rate (increased elution temperature) for some randomly chosen compounds from MegaMix A and MegaMix B. Figure 3.5(B) shows the average variations of all compounds from MegaMix and MegaMix B at different elution temperature. Figure 3.5(B) shows that, generally, retention index variation increased with increased elution temperature i.e. increasing ramp rate. However, MegaMix B showed comparatively higher variations at ramp rate $3.5^{\circ} \mathrm{C} / \mathrm{min}$ in comparison with its adjacent ramp rate that could be due to the experimental variations. By design, the transfer line located between the secondary oven and the mass spectrometer was set at $280{ }^{\circ} \mathrm{C}$ during the entire experiment. 
A.

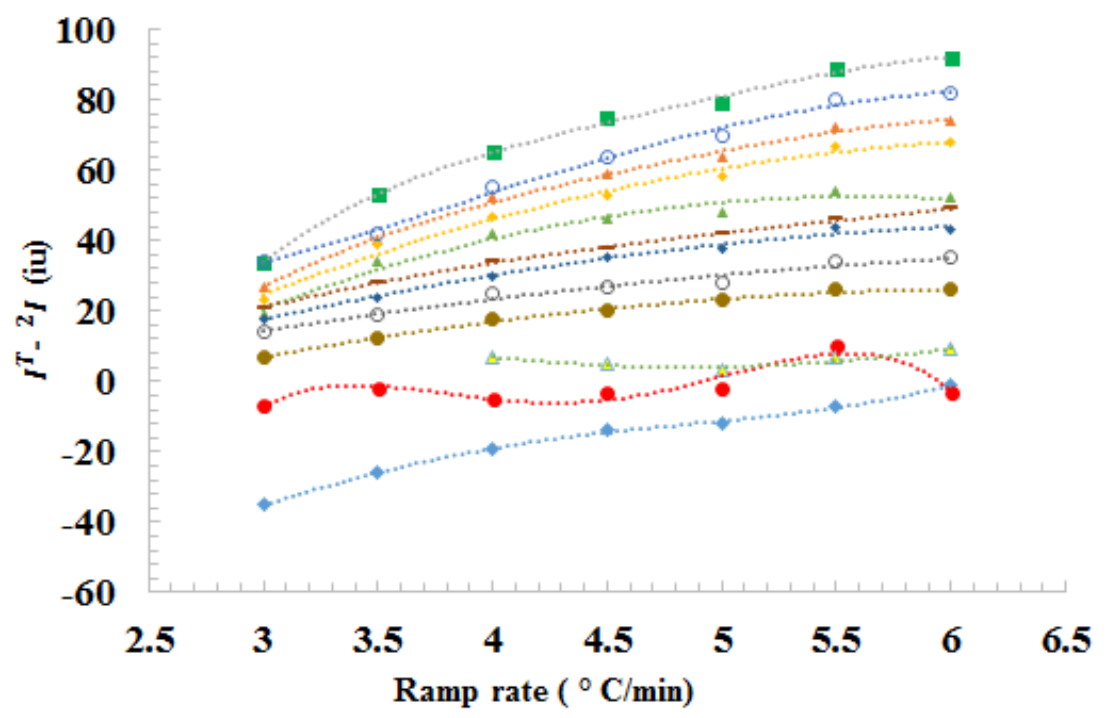

B.

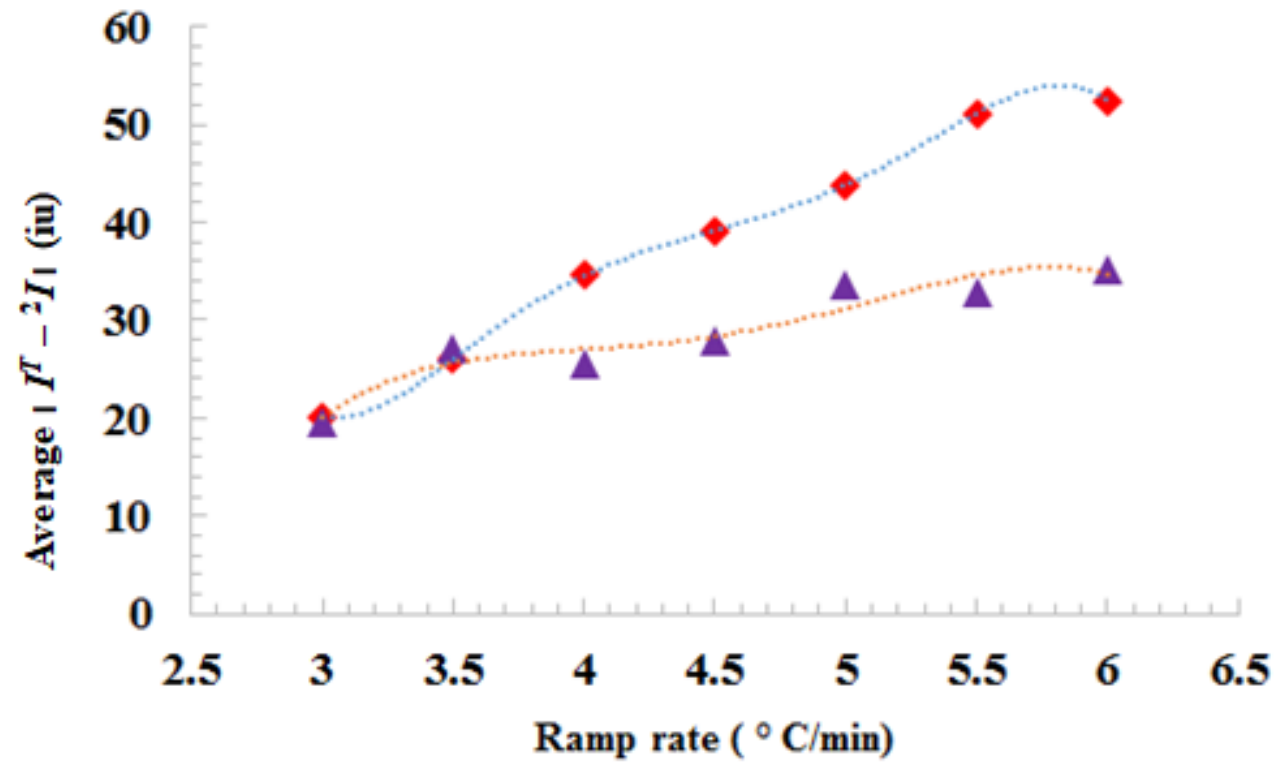


Figure 3.5. Effect of elution temperature on retention index calculation. (A) Some randomly selected compounds from MegaMix A and MegaMix B at different ramp rate. (B) Average $\mid I^{T}{ }^{2} I I$ (iu) for MegaMix A (red square) and MegaMix (violet triangle) B at different ramp rate.

The effect of this fixed temperature on the magnitude of ${ }^{2} I$ was different from compound to compound as each compound eluted at different temperature.

\subsubsection{Robustness of Surface Fitting to Variations of Instrumental Settings}

To investigate the reproducibility and the robustness of the developed method in analyzing GC×GC-MS data, we analyzed the sixteen in-house made mixtures, MegaMix A, and MegaMix B under different instrumental conditions by varying the temperature offset of the secondary oven, the ramp rate of temperature gradient, and modulation period.

Tables 3.4, 3.8 and 3.9 respectively lists the ${ }^{2} I$ of the compounds identified from the six mixtures that were not derivatized, from MegaMix A and from MegaMix B. An average $R S D$ of the calculated ${ }^{2} I$ for the compound standards in the six in-house made mixtures, MegaMix A and MegaMix B was $0.28 \%, 0.46 \%$ and $0.34 \%$, respectively. The small RSD of ${ }^{2} I$ demonstrates that the developed method is robust in calculating the second dimension retention index of compounds analyzed in different instrumental conditions. 
Table 3.8. The second dimension retention indices of compounds identified from MegaMix A

\begin{tabular}{|c|c|c|c|c|c|c|c|c|c|}
\hline Chromatographic conditions & & & & (iu) ${ }^{a}$ & & & & & $I^{T}$ from \\
\hline Secondary oven offset $\left({ }^{\circ} \mathrm{C}\right)$ & +5 & +5 & +5 & +5 & +5 & +5 & +5 & +10 & GC-MS \\
\hline Ramp rate $\left({ }^{\circ} \mathrm{C} /\right.$ minute $)$ & 3 & 3.5 & 4 & 4.5 & 5 & 5.5 & 6 & 5 & \\
\hline Modulation period (s) & 5 & 5 & 5 & 5 & 5 & 5 & 5 & 4 & \\
\hline N-Nitrosodimethylamine & 967 & 968 & 971 & 972 & 973 & 975 & 974 & 975 & 952 \\
\hline Phenol & 1170 & 1175 & 1182 & 1186 & 1188 & 1191 & 1193 & 1195 & 1157 \\
\hline 2-Chlorophenol & 1180 & 1186 & 1192 & 1197 & 1200 & 1206 & 1205 & 1207 & 1162 \\
\hline Bis(2-chloroethyl) ether & 1187 & 1192 & 1198 & 1200 & 1203 & 1206 & 1206 & 1208 & 1180 \\
\hline 2,2'-Oxybis(2-chloropropane) & 1215 & 1222 & 1226 & 1227 & 1228 & 1231 & 1230 & 1231 & 1210 \\
\hline Aniline & 1215 & 1221 & 1229 & 1234 & 1238 & 1242 & 1245 & 1245 & 1203 \\
\hline Hexachloroethane & 1238 & 1245 & 1252 & 1255 & 1259 & 1260 & 1263 & 1260 & 1215 \\
\hline 2-Methylphenol & 1255 & 1260 & 1267 & 1269 & 1272 & 1277 & 1277 & 1278 & 1242 \\
\hline Benzyl alcohol & 1261 & 1267 & 1275 & 1279 & 1281 & 1286 & 1288 & 1289 & 1250 \\
\hline N-nitrosodipropylamine & 1271 & 1275 & 1281 & 1281 & 1285 & 1288 & 1288 & 1290 & 1260 \\
\hline 3-Methylphenol & 1277 & 1281 & 1288 & 1291 & 1292 & 1297 & 1297 & 1300 & 1263 \\
\hline Isophorone & 1337 & 1342 & 1348 & 1352 & 1354 & 1356 & 1359 & 1361 & 1324 \\
\hline 2,4-Dimethylphenol & 1359 & 1364 & 1370 & 1372 & 1373 & 1379 & 1380 & 1382 & 1345 \\
\hline 2-Nitrophenol & 1376 & 1384 & 1394 & 1398 & 1401 & 1406 & 1410 & 1409 & 1358 \\
\hline Bis(2-chloroethoxy)-Methane & 1387 & 1393 & 1398 & 1400 & 1401 & 1406 & 1406 & 1408 & 1375 \\
\hline 2,4-DichloroPhenol & 1391 & 1397 & 1404 & 1407 & 1410 & 1417 & 1419 & 1420 & 1367 \\
\hline 4-Chloroaniline & 1509 & 1502 & 1511 & 1520 & 1524 & 1529 & 1532 & 1532 & 1477 \\
\hline $\begin{array}{l}\text { 1,3-Cyclopentadiene, } \\
\text { 1,2,3,4,5,5-hexachloro- }\end{array}$ & 1526 & 1529 & 1532 & 1537 & 1541 & 1546 & 1547 & 1551 & 1492 \\
\hline 4-Chloro-3-methylphenol & 1542 & 1549 & 1555 & 1559 & 1563 & 1567 & 1570 & 1571 & 1521 \\
\hline 2-Methylnaphthalene & 1557 & 1567 & 1574 & 1580 & 1584 & 1590 & 1593 & 1594 & 1561 \\
\hline 1-Methylnaphthalene & 1588 & 1598 & 1608 & 1613 & 1620 & 1626 & 1627 & 1628 & 1581 \\
\hline 2,4,6-Trichlorophenol & 1613 & 1622 & 1629 & 1634 & 1636 & 1641 & 1647 & 1654 & 1648 \\
\hline
\end{tabular}


2,4,5-Trichlorophenol

2-Chloronaphthalene

2-Nitroaniline

Dimethyl phthalate

Acenapthylene

1,4-Dinitrobenzene

Acenaphthene

2,6-Dinitrotoluene

1,3-Dinitrobenzene

Dibenzofuran

2,3,5,6-Tetrachlorophenol

2,3,4,6-Tetrachlorophenol

1,2-Dinitrobenzene

2,4-dinitro-phenol

3-Nitroaniline

4-Chlorophenyl phenyl ether

$\begin{array}{rllllllll} & 1628 & 1635 & 1640 & 1643 & 1651 & 1654 & 1655 & \\ 1669 & 1680 & 1691 & 1696 & 1702 & 1709 & 1711 & 1711 & 1640 \\ 2178 & 1857 & 1804 & 1812 & 1824 & 1834 & 1838 & 1835 & 1761 \\ 1867 & 1805 & 1810 & 1820 & 1826 & 1834 & 1831 & 1834 & 1776 \\ 1835 & 1798 & 1812 & 1824 & 1833 & 1841 & 1845 & 1843 & 1785 \\ 2327 & 1921 & 1851 & 1850 & 1862 & 1874 & 1877 & 1874 & 1806 \\ 1835 & 1831 & 1844 & 1855 & 1862 & 1871 & 1872 & 1873 & \\ 2087 & 1935 & 1852 & 1860 & 1870 & 1881 & 1884 & 1880 & 1823 \\ 2481 & 1946 & 1858 & 1863 & 1874 & 1884 & 1888 & 1883 & 1816 \\ 1853 & 1861 & 1874 & 1883 & 1889 & 1899 & 1901 & 1901 & 1819 \\ 1857 & 1870 & 1880 & 1887 & 1893 & 1900 & 1901 & 1905 & 1828 \\ 1870 & 1883 & 1895 & 1902 & 1907 & 1915 & 1917 & 1925 & 1843 \\ 2510 & 2154 & 1939 & 1905 & 1915 & 1929 & 1936 & 1930 & 1858 \\ & 1907 & 1906 & 1918 & 1928 & 1936 & 1937 & 1936 & 1874 \\ 2589 & 2061 & 1931 & 1924 & 1936 & 1951 & 1957 & 1953 & 1910 \\ 1913 & 1929 & 1937 & 1943 & 1948 & 1957 & 1958 & 1960 & 1890 \\ 2199 & 1999 & 1928 & 1940 & 1950 & 1961 & 1961 & 1960 & 1890 \\ 1933 & 1935 & 1946 & 1954 & 1956 & 1962 & 1961 & 1965 & 1914 \\ 1948 & 1951 & 1962 & 1975 & 1984 & 1992 & 1994 & 1996 & \\ 1968 & 1979 & 1991 & 2001 & 2006 & 2018 & 2015 & 2017 & 1941 \\ 2015 & 1999 & 2009 & 2020 & 2027 & 2036 & 2040 & 2039 & 1972 \\ 2048 & 2010 & 2020 & 2033 & 2041 & 2053 & 2053 & 2051 & 1979 \\ 2032 & 2051 & 2063 & 2073 & 2077 & 2087 & 2090 & 2093 & 1998 \\ 2052 & 2064 & 2078 & 2086 & 2092 & 2102 & 2102 & 2104 & 2023 \\ 2125 & 2140 & 2155 & 2166 & 2174 & 2184 & 2188 & 2188 & 2064 \\ 2359 & 2247 & 2244 & 2263 & 2277 & 2294 & 2299 & 2294 & 2174 \\ 2395 & 2251 & 2254 & 2274 & 2287 & 2301 & 2307 & 2301 & 2185 \\ 2300 & 2315 & 2323 & 2327 & 2329 & 2335 & 2333 & 2340 & 2281 \\ 3228 & 2540 & 2373 & 2375 & 2391 & 2412 & 2420 & 2411 & 2300 \\ 2568 & 2573 & 2570 & 2572 & 2573 & 2585 & 2572 & 2592 & 2575\end{array}$




\begin{tabular}{|c|c|c|c|c|c|c|c|c|c|}
\hline Fluoranthene & 3090 & & 2625 & 2636 & 2658 & 2680 & 2688 & 2678 & \\
\hline Pyrene & 3973 & 2713 & 2755 & 2732 & 2752 & 2777 & 2791 & 2777 & \\
\hline Bis(2-ethylhexyl) phthalate & 2859 & 2874 & 2871 & 2870 & 2872 & & & & 2832 \\
\hline Benzyl butyl phthalate & 3008 & 2878 & 2879 & 2898 & 2911 & 2929 & 2924 & 2924 & 2823 \\
\hline
\end{tabular}

${ }^{a}$ Blanks means either the compound was not correctly identified or it eluted at $290{ }^{\circ} \mathrm{C}$ (isothermal condition in ${ }^{2} T_{e}$ )

${ }^{\mathrm{b}}$ Italic values were considered as outliers and were not included in $R S D$ calculation. Those compounds had a very large second dimension retention index at ramp rate $3{ }^{\circ} \mathrm{C} /$ minute and $4{ }^{\circ} \mathrm{C} /$ minute compared to their retention index calculated in other ramp rates

Table 3.9. The second dimension retention indices of compounds identified from MegaMix B

\begin{tabular}{|c|c|c|c|c|c|c|c|c|}
\hline Chromatographic conditions & & & & ${ }^{2} I(\mathrm{iu})^{\mathrm{a}}$ & & & & $I^{T}$ from \\
\hline Secondary oven offset $\left({ }^{\circ} \mathrm{C}\right)$ & +5 & +5 & +5 & +5 & +5 & +5 & +5 & +10 GC-MS \\
\hline Ramp rate $\left({ }^{\circ} \mathrm{C} /\right.$ minute $)$ & 3 & 3.5 & 4 & 4.5 & 5 & 5.5 & 6 & 5 \\
\hline Modulation period (s) & 5 & 5 & 5 & 5 & 5 & 5 & 5 & 4 \\
\hline $\begin{array}{l}\text { cis-1,3-Dichloro-1- } \\
\text { Propene, }(\mathrm{Z})\end{array}$ & 872 & 875 & 872 & 871 & 882 & & & 869 \\
\hline trans-1,3-Dichloro-Propene€ & & & 913 & 912 & 915 & 911 & 912 & 910 \\
\hline Toluene & 887 & 891 & 883 & 883 & 894 & & & 882 \\
\hline 1,1,2-Trichloroethane & & 930 & 925 & 924 & 924 & 925 & 925 & 920 \\
\hline Ethyl methacrylate & & & 888 & 889 & 897 & & & 887 \\
\hline 1,3-Dichloropropane & 942 & 949 & 943 & 943 & 946 & 943 & 943 & 940 \\
\hline Dibromochloro-Methane & 973 & 977 & 975 & 975 & 979 & 977 & 977 & 975 \\
\hline Tetrachloroethene & 911 & 915 & 910 & 909 & 913 & & & 909 \\
\hline 1,2-Dibromoethane & 985 & 990 & 988 & 990 & 992 & 990 & 992 & 987 \\
\hline Chlorobenzene & 999 & 1007 & 1002 & 1002 & 1004 & 1005 & 1004 & 1002 \\
\hline $1,1,1,2$-Tetrachloroethane & 997 & 1001 & 986 & 994 & 997 & 999 & 995 & 996 \\
\hline
\end{tabular}




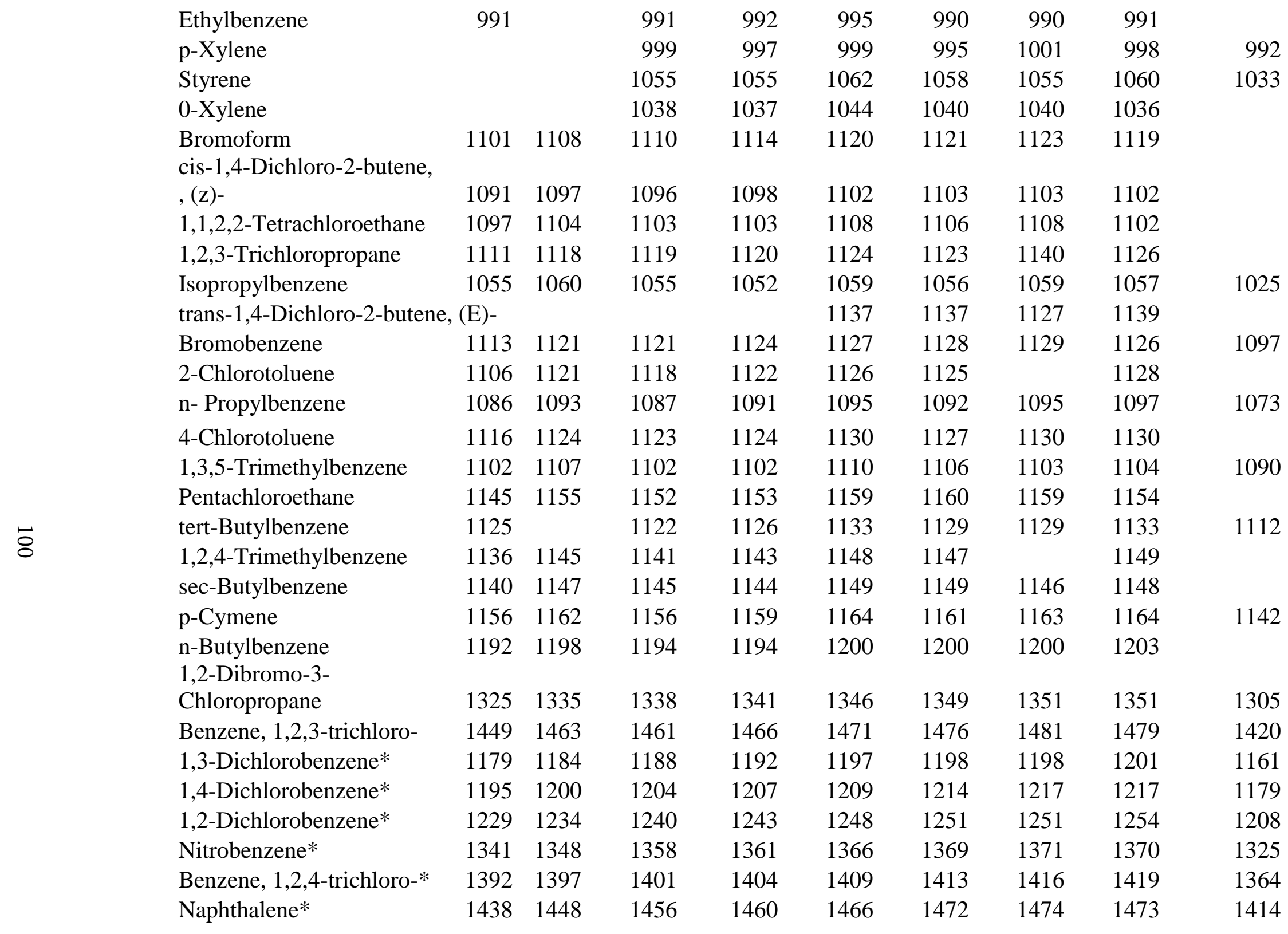


1,3-Butadiene, 1,1,2,3,4,4hexachloro-*

${ }^{a}$ Blank means the compound was not identified 


\subsubsection{Comparison with ${ }^{2}$ I Calculated by Pseudo-isothermal Condition}

Chapter Two reported a surface fitting method for the calculation of ${ }^{2} I$ by adopting the conventional concept, i.e., approximating the second dimension separation in $\mathrm{GC} \times \mathrm{GC}$ MS as in pseudo-isothermal condition, and demonstrated that the method can reduce the variation introduced by conventional isovolatility curve approach.$^{87}$ In that study, we analyzed the $n$-alkanes in isothermal mode at 46 different temperatures. After deducting the temperature-dependent second dimension hold-up time ${ }^{2} t_{M}$ from each retention time ${ }^{2} t_{R}$, we obtained the adjusted ${ }^{2} t_{R}^{\prime}$ for each data point. We then constructed the ${ }^{2} I_{S}$ using the adjusted ${ }^{2} t_{R}^{\prime}$ and ${ }^{2} T_{e}$ of $n$-alkanes. In the current study, the $n$-alkanes were analyzed in temperature-programmed mode at 16 ramp rates. The ${ }^{2} t_{R}$ and ${ }^{2} T_{e}$ values of $n$-alkanes were directly used to construct the ${ }^{2} I_{s}$, where the calculation adjusted retention time ${ }^{2} t_{R}^{\prime}$ and hold-up time ${ }^{2} t_{M}$ was eliminated.

Figure 3.6 shows the distribution of the deviation $\left|I^{T}-{ }^{2} I\right|$ of the 322 compounds, where ${ }^{2} I_{a}$ was calculated using the method developed in this study and ${ }^{2} I_{b}$ was calculated using the method developed in our previous study. ${ }^{87}$ The average deviation of the 322 compounds was 30.4 iu using the method developed in our previous study, where the second dimension separation was considered as in pseudo-isothermal condition. The average deviation of the 322 compound was reduced to 18.0 iu using the method developed in the current study. 
A.

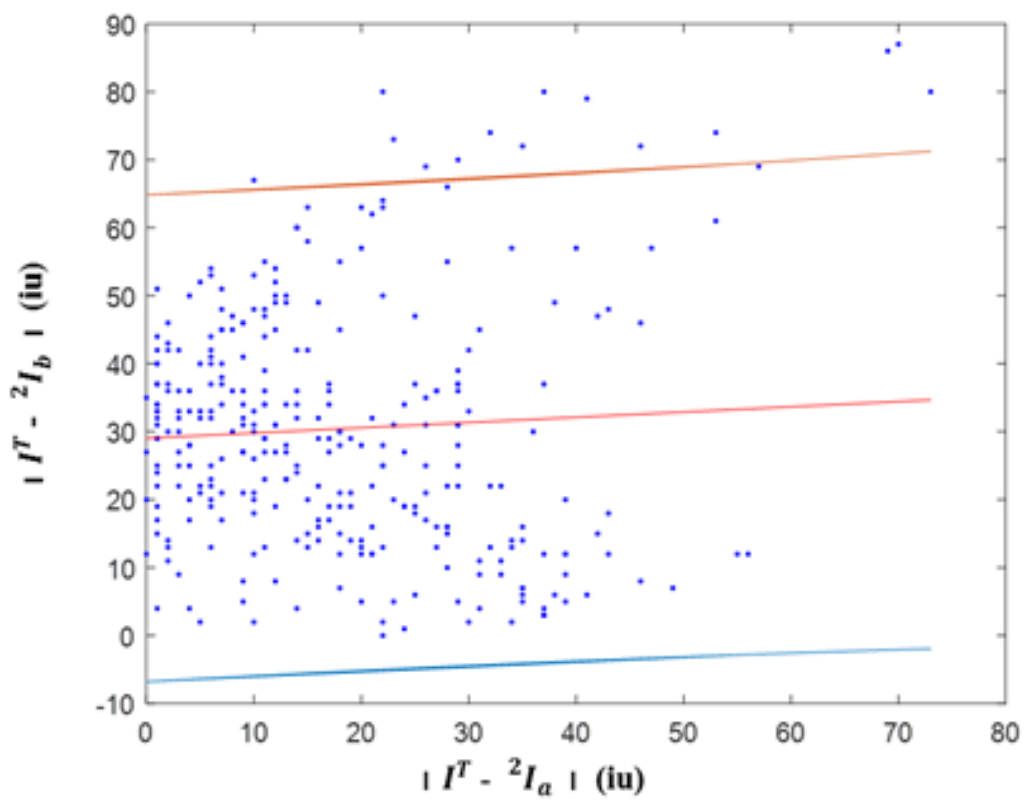

B.

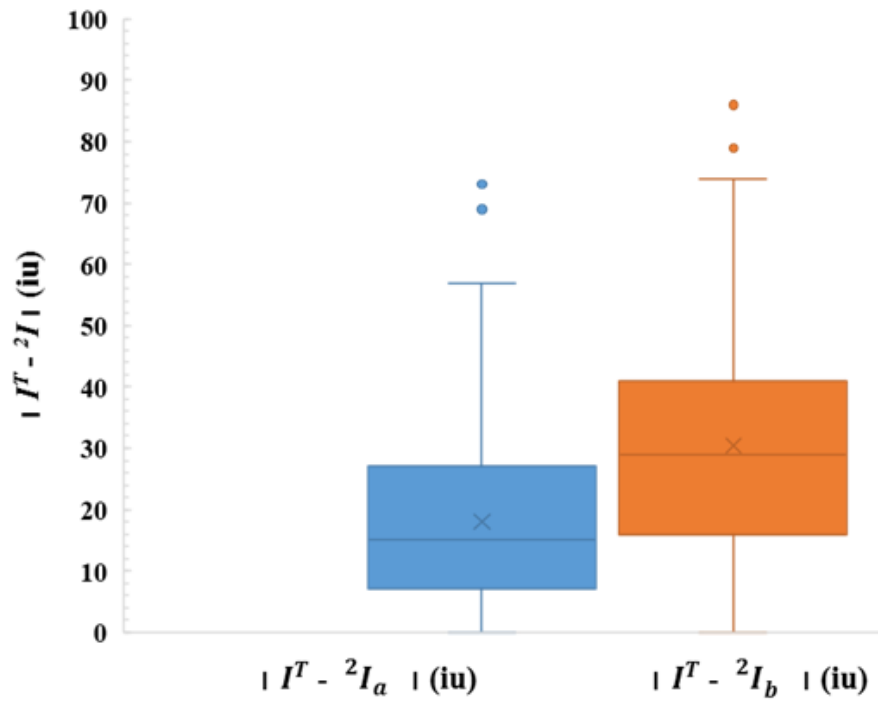


Figure 3.6. Effect of hold-up time calculation on the deviation $\left|I^{T}-{ }^{2} I\right| .{ }^{2} I_{a}$ is the second dimension retention index calculated by without involving hold-up time calculation. ${ }^{2} I_{b}$ is the second dimension retention index calculated by involving hold-up time calculation. (A) Distribution of $\left|I^{T}-{ }^{2} I\right|$ by approximating the second dimension separation as in pseudo-isothermal mode vis. $\left|I^{T}-{ }^{2} I\right|$ of considering the second dimension separation in its actuality, i.e., temperature-programmed mode. The red line shows the result of linear fitting while the blue and pink lines are fitting confidence with 95\% confidence interval. (B) Box plot of $\left|I^{T}-{ }^{2} I\right|$ calculated using the two methods.

In order to study the robustness of the developed method, the six mixtures that were not derivatized, the mixture of MegaMix A and MegaMix B, were respectively analyzed in different instrumental settings. We also calculated the ${ }^{2} I$ of those compounds using the method developed in our previous study. The average $R S D$ of the ${ }^{2} I$ for the compounds in the six in-house made mixtures, MegaMix A and MegaMix B is $0.31 \%, 0.63 \%$ and $0.42 \%$, respectively. However, the corresponding average $R S D$ of the ${ }^{2} I$ calculated using the method developed in this study for the compounds in the six mixtures, MegaMix A and MegaMix B is $0.28 \%, 0.46 \%$ and $0.34 \%$, respectively.

The higher deviation of the ${ }^{2} I$ from corresponding $I^{T}$, as well as the increased average $R S D$ in the previous method were caused by the concept of approximating the second dimension separation as pseudo-isothermal, which therefore involved the calculation of adjusted retention time ${ }^{2} t_{R}^{\prime}$ and hold-up time ${ }^{2} t_{M}$. While difference methods have been developed to estimate ${ }^{2} t_{M}$, each of those methods introduces a certain degree of variation that was propagated to the calculation of ${ }^{2} t_{R}^{\prime}$ and ${ }^{2} I$. 
We also investigated the effect of the polynomial model selection for surface fitting model. In our previous study, i.e., the method including hold-up time calculation, the optimal fitting model was "poly 25 " but in our current study it is "poly35". Therefore, we calculated the ${ }^{2} I$ of 80 compound standards, MSTFA and MTBSTFA derivatized compounds, MegaMix A and MegaMix B using both "poly25" and "poly35" surface fitting model, and found that the average ${ }^{2} I$ difference between these two method only $1.1 \mathrm{iu}$. Therefore, the larger variations was solely introduced during the hold-up time calculation. Furthermore, our previous method used 46 chromatographic analysis of $n$-alkanes at different temperatures in isothermal mode to calculate the ${ }^{2} t_{M}$, while the current method used only 16 chromatographic runs. In summary, the method developed in this study is much simpler and outperforms our previous method as well as the conventional isovolatility curve approach for the calculation of the second dimension retention index.

\subsection{Conclusions}

This study was a continuation of our previous work on calculating the second dimension retention index in GCxGC-MS via surface fitting. The method developed in this study considered the second dimension separation as its actuality, i.e., in temperatureprogrammed mode, and directly calculated the second dimension retention index via surface fitting. This approach eliminated the concept of approximating the second dimension separation as in pseudo-isothermal condition. Therefore, the current method does not involve the calculation of temperature dependent second dimension hold-up time ${ }^{2} t_{M}$, resulting in increased accuracy of the second dimension retention index calculation. Analysis of a wide range of chemicals ranging from very non-polar to polar compounds as well as biologically important compounds such as amino acids and organic 
acids showed that the deviation of ${ }^{2} I$ values from their corresponding linear retention index $I^{T}$ calculated from GC-MS data is within the normal deviation range, thus showing the effectiveness of the developed method. Compared with the existing approaches, the method developed is much more accurate in calculating the second dimension retention index in GC $\times$ GC-MS. Despite the unique advantage of the developed method over the existing method, the developed method has limited separation map coverage compared with the method developed in Chapter Two where isothermal modes of GC×GC-MS were used. In the current developed method, for the second dimension retention index surface construction, reference compounds were analyzed under temperature gradient mode. While very slow temperature gradient $\left(0.85^{\circ} \mathrm{C}\right)$ was used, the separation map was still not sufficient to cover the separation region of some compounds. Therefore, few compounds from MegaMix A at ramp rate $3{ }^{\circ} \mathrm{C}$ fell outside of the second dimension retention index surface and showed very high ${ }^{2} I$ deviations from their retention index measured at GCMS. Future endeavor should focus on the development of method for increasing the second dimension retention map coverage under temperature-programmed mode of GC×GC-MS. 


\section{CHAPTER 4}

\section{UNIVERSAL REFERENCE SYSTEM FOR CALCULATING THE SECOND DIMENSION RETENTION INDEX IN COMPREHENSIVE TWO-DIMENSIONAL GAS CHROMATOGRAPHY MASS SPECTROMETRY}

\subsection{Introduction}

Comprehensive two-dimensional gas chromatography mass spectrometry (GC×GCMS) is an excellent tool for chemical profiling of complex samples owing to its superior separation capacity, selectivity, sensitivity and dynamic range. ${ }^{59-61,63,82,96}$ While mass spectrum matching is widely used for compound identification in analyzing GC $\times$ GC-MS data $^{83,84,97}$, unambiguous identification of compounds is still a challenge due to the similar mass spectra of different compounds, e.g., homologous compounds or isomers. ${ }^{85}$ Incorporating compound separation information with mass spectrum matching can significantly improve the accuracy of compound identification. ${ }^{98}$

GC $\times$ GC-MS provides two pieces of chromatographic separation information including first dimension retention time $\left({ }^{1} t_{R}\right)$ and the second dimension retention time $\left({ }^{2} t_{R}\right)$. Retention time cannot be used directly as there is always a retention time variation between instrument to instrument, run-to run and among the different laboratory using 
different kinds of instrument. In order to usecompound separation information for compound identification and avoid the variations, the first dimension retention time $\left({ }^{1} t_{R}\right)$ and the second dimension retention time $\left({ }^{2} t_{R}\right)$ of a compound in GC×GC-MS must be normalized. Conventionally, analytes' retention times are converted into retention index by using a homologous reference series predominantly $n$-alkane homologous series. However, $n$-alkanes have poor separation in a polar column and result in a poor reproducibility in calculating the retention index. Therefore, some other reference series has been used as reference series. For instance, fatty acid methyl esters (FAME) homologous series have been employed to calculate the retention index using a retention index scale different from the $n$-alkanes scale. Specifically, the FAME based scale is based on the calculation of the equivalent chain length, carbon number and modified equivalent chain length. ${ }^{99}$ Moreover, some other homologous series, i.e., 2-ketones ${ }^{100,101}$, n-propyl ester $^{102}$, alkanoate esters ${ }^{103}$, alkanols and acetates ${ }^{104}$ etc, have also been employed. Another type of retention index system was defined based on the series of polycyclic aromatic hydrocarbon (PAH) standard compounds including naphthalene, Phenanthrene, chrysene and pentacene to calculate the retention index of polyaromatic compounds..$^{95}$

In GC $\times \mathrm{GC}-\mathrm{MS}$, the first dimension separation is usually operated under temperatureprogrammed mode and the ${ }^{1} t_{R}$ is converted into a linear retention index $\left(I^{T}\right)$ using the Van Den Doll and Kratz equation. ${ }^{28}$ The second dimension separation is often considered as being in pseudo-isothermal mode as the temperature change within a modulation cycle is negligible ${ }^{65-71}$, and the ${ }^{2} t_{R}$ is converted into Kováts retention index ${ }^{2} I{ }^{29}$ However, the calculation of ${ }^{2} I$ is not straightforward because the temperature of the second dimension column varies at each modulation period $\left(P_{M}\right)$ during $\mathrm{GC} \times \mathrm{GC}-\mathrm{MS}$ analysis. 
Several methods have been developed to calculate the ${ }^{2} I$ from the ${ }^{2} t_{R}$, such as isovolatility curve and retention index surface approaches..$^{40,41,73-76,93,105}$ In the isovolatility curve approach, the hold-up time ${ }^{2} t_{M}$ at different temperatures of the second dimension column was first estimated for the calculation of adjusted retention time, ${ }^{2} t_{R}^{\prime}={ }^{2} t_{R}-$ ${ }^{2} t_{M}$. The ${ }^{2} t_{R}^{\prime}$ s of the same reference compound at different column temperatures were then used to construct the isovolatility curve of that reference compound. The isovolatility curves of all reference compounds formed the second dimension retention map (i.e., the two-dimensional separation space formed by ${ }^{2} t_{R}$ and ${ }^{2} T_{e}$ of all reference compounds), and the ${ }^{2} I$ value of a compound was finally calculated from the two adjacent isovolatility curves of two reference compounds. Our group proposed a second dimension retention index surface approach for calculation of ${ }^{2} I$ value of each compound using $n$-alkanes as reference compounds ${ }^{105}$, where the adjusted second dimension retention time ${ }^{2} t_{R}^{\prime}$ and the second dimension column temperature ${ }^{2} T_{e}$ formed an X-Y plane and the ${ }^{2} I$ was the Zaxis. The second dimension retention index surface, ${ }^{2} I_{S}$, was constructed using the ${ }^{2} I$ values of all reference compounds via a surface fitting model. The ${ }^{2} I$ of an analyte measured by GCXGC-MS was then calculated from ${ }^{2} I_{S}$ based on its ${ }^{2} t_{R}^{\prime}$ and ${ }^{2} T_{e}$.

In order to use the two-dimensional retention index of $\mathrm{GC} \times \mathrm{GC}-\mathrm{MS}$ to aid compound identification in analysis of complex samples, the second dimension retention map of reference compounds should encompass the values of ${ }^{2} t_{R}$ and ${ }^{2} T_{e}$ of all compounds in a sample. The separation of reference compounds by $\mathrm{GC} \times \mathrm{GC}$ is mainly determined by column chemistry and the chemical property of the reference compounds, i.e., interaction between the column stationary phase and the reference compounds. The most widely used 
reference series for calculating the second dimension retention index is $n$-alkanes, which are non-polar compounds. $n$-Alkanes are the ideal reference compounds for non-polar and mid-polar compounds, but not for polar compounds, as the polar compounds elute much later than $n$-alkanes in a polar column. In that case, the second dimension retention map of $n$-alkanes is not large enough to cover the polar compounds and therefore, cannot be directly used to calculate the ${ }^{2} I$ of the polar analytes. A solution to that problem is to extrapolate the second dimension retention map of the reference compounds. However, extrapolation introduces a large degree of variation in calculation of ${ }^{2} I$. Another approach is to use a polar reference series (e.g., alcohols) for the polar column and a non-polar reference series for the non-polar column. ${ }^{75}$ However, this method raises a problem of retention index scaling. Alcohols generate ${ }^{2} I$ values in alcohol scale, but most of the existing retention index databases contain retention indices that use $n$-alkanes as references, i.e., in $n$-alkane retention index scale. For example, the National Institute of Standards and Technology MS database (NIST 2014) contains Kovats and LRI data for 385872 and 82337 compounds, respectively.

The objective of this work was to construct a large second dimension retention map for reference compounds in $\mathrm{GC} \times \mathrm{GC}-\mathrm{MS}$ using $\mathrm{C}_{4}-\mathrm{C}_{25}$ fatty acid methyl esters $\left(\mathrm{C}_{4}-\mathrm{C}_{25}\right.$ FAMEs) as a reference series instead of conventional $n$-alkane based reference series that covers non-polar to polar compounds. The second objective of this word was to develop a method to calculate the ${ }^{2} I$ in second dimension separation space using $\mathrm{C}_{4}-\mathrm{C}_{25}$ FAMEs while keeping the retention index scale in $n$-alkane scale. We developed a surface fitting method using $\mathrm{C}_{4}-\mathrm{C}_{25}$ FAMEs as reference compounds and considered the second dimension separation in $\mathrm{GC} \times \mathrm{GC}-\mathrm{MS}$ as linear temperature-programmed separation 
(unpublished results. Performance of the developed method was then tested and validated by analyzing $n$-alkanes, 80 standard compounds and two commercially available mixtures (i.e., MegaMix A and MegaMix B) with their corresponding linear retention indices on the same column measured by GC-MS.

\subsection{Experimental Section}

\subsubsection{Sample Preparation}

A total of 80 compound standards and 4 commercial mixtures were purchased in this study. Six in-house made solutions were prepared by mixing the 80 compound standards in different combinations, including a solution containing odd number of $\mathrm{C}_{5}-\mathrm{C}_{23}$ FAMEs, a solution containing $\mathrm{C}_{4}-\mathrm{C}_{24}$ (both even and odd number) carbon saturated FAMEs, a solution containing $\mathrm{C}_{5}-\mathrm{C}_{22}$ saturated fatty acids, a 2-ketone mixture, a $n$-alcohol mixture, an 1-iodoalkane mixture, and an aldehyde mixture. All these six mixtures were prepared in dichloromethane with a concentration of $100 \mu \mathrm{g} / \mathrm{mL}$ per compound. MegaMix A and MegaMix B were prepared in dichloromethane at a concentration of $50 \mu \mathrm{g} / \mathrm{mL}$ per compound.

\subsubsection{GCXGC-MS Experiments}

The LECO (St. Joseph, MI) Pegasus® 4D GC $\times$ GC-MS was configured with a Gerstel MPS2 autosampler and an Agilent 6890 gas chromatograph featuring a LECO two-stage cryogenic modulator and a secondary oven. The first dimension column was a non-polar DB-5ms (phenyl arylene polymer virtually equivalent to a (5\%-phenyl)methylpolysiloxane) $60.0 \mathrm{~m} \times 0.25 \mathrm{~mm}^{1} d_{c} \times 0.25 \mu \mathrm{m}^{1} d_{f}$ column, and the second dimension column was a medium polar BD-17ms ((50\%-phenyl)-methylpolysiloxane) 1.0 
$\mathrm{m} \times 0.1 \mathrm{~mm}{ }^{2} d_{c} \times 0.1 \mu \mathrm{m}^{2} d_{f}$ column. Both columns were obtained from Agilent Technologies (Santa Clara, CA), and were connected by means of a press-fit connector before the thermal modulator. The flow rate of ultra-high purity helium carrier gas $(99.999 \%$ ) was set to $2.0 \mathrm{~mL} / \mathrm{min}$ at a corrected constant flow via pressure ramps with an initial head pressure of 31.9 psi. The MS parameters were as follows: MS range 45-800 $\mathrm{m} / \mathrm{z}$; data acquisition rate 200 spectra/s; temperature of ion source chamber $230{ }^{\circ} \mathrm{C}$; temperature of MS transfer line $280^{\circ} \mathrm{C}$; detector voltage $1641 \mathrm{~V}$; electron energy $70 \mathrm{eV}$. After a solvent delay of $245 \mathrm{~s}$, the acceleration voltage was turned on.

In order to construct the second dimension retention index surface, the reference mixture containing $\mathrm{C}_{4}-\mathrm{C}_{25}$ FAMEs were analyzed by $\mathrm{GC} \times \mathrm{GC}-\mathrm{MS}$ seventeen times in temperature-programmed mode using different ramp rates, including 0.85, 1.00, 1.13, 1.25, $1.50,1.65,1.75,2.00,2.50,3.00,3.50,4.00,4.50,5.00,5.50,6.00$, and $7.00{ }^{\circ} \mathrm{C} / \mathrm{min}$. A 1 $\mu \mathrm{L}$ sample was injected into the inlet chamber of the GC $\times \mathrm{GC}-\mathrm{MS}$ system and the split ratio was set at 10:1. The inlet temperature was $280{ }^{\circ} \mathrm{C}$. The thermal modulator and the secondary oven were set to $+20{ }^{\circ} \mathrm{C}$ and $+5{ }^{\circ} \mathrm{C}$ relative to the primary oven, respectively. Initial temperature of the first dimension column ${ }^{1} T_{e}$ was set at $60{ }^{\circ} \mathrm{C}$ which was kept for 1 minute and then ramped to $285{ }^{\circ} \mathrm{C}$ using different ramp rates. ${ }^{l} T_{e}$ was kept at $285{ }^{\circ} \mathrm{C}$ for 13 minutes to let all compounds elute off the column. The second dimension column was also set under same conditions but at $+5^{\circ} \mathrm{C}$ with respect to the first dimension column. $P_{M}$ was set as $6 \mathrm{~s}$.

In order to validate the developed method, $\mathrm{C}_{7}-\mathrm{C}_{30} n$-alkanes were analyzed by GC $\times$ GC-MS seventeen times in identical chromatographic conditions as used for the reference compounds, $\mathrm{C}_{4}-\mathrm{C}_{25}$ FAMEs. To test the reproducibility of the developed method, 
the six in-house made mixtures were each analyzed five times in temperature-programed mode using different ramp rates, i.e. $4.00,4.50,5.00,5.50$ and $6.00{ }^{\circ} \mathrm{C} / \mathrm{min}$. To study the robustness of the surface fitting method, the MegaMix A and MegaMix B mixtures were respectively analyzed under different instrumental conditions by varying the temperature offset of the secondary oven from +5 to $+10^{\circ} \mathrm{C}$, the ramp rate of temperature gradient from 4.0 to $6.0{ }^{\circ} \mathrm{C} / \mathrm{min}$, and the modulation period from 4 to $5 \mathrm{~s}$. The other instrumental parameters were kept the same as described above.

\subsubsection{GC-MS Experiments}

All six in-house made mixtures, MegaMix A and MegaMix B were respectively analyzed on a Thermo ITQ 1100 Ion Trap MS equipped with a Trace 1310 GC and an AI 1310 auto sampler. The column was a DB-17ms capillary column $\left(45.0 \mathrm{~m} \times 0.1 \mathrm{~mm}^{2} d_{c} \times\right.$

$0.1 \mu \mathrm{m}^{2} d_{f}$, (50\%-phenyl)-methylpolysiloxane) purchased from Agilent Technologies (Agilent Technologies J\&W, Santa Clara, CA, USA). The helium carrier gas $(99.999 \%$ purity) flow rate was set to $2.0 \mathrm{~mL} / \mathrm{min}$, and the inlet temperature was $280{ }^{\circ} \mathrm{C}$. The column temperature was programmed with an initial temperature of $60{ }^{\circ} \mathrm{C}$ for 1.0 minute, ramped at $5{ }^{\circ} \mathrm{C} / \mathrm{min}$ to $290^{\circ} \mathrm{C}$, and finally maintained at $290{ }^{\circ} \mathrm{C}$ for 13 minute. The mass range was set as $29-800 \mathrm{~m} / \mathrm{z}$. The ion source chamber was $230{ }^{\circ} \mathrm{C}$ with a transfer line temperature of $280{ }^{\circ} \mathrm{C}$.

A mixture of $\mathrm{C}_{7}-\mathrm{C}_{30} n$-alkanes at a concentration of $75 \mu \mathrm{g} / \mathrm{mL}$ was also analyzed by GC-MS and the retention times of these $n$-alkanes were used to calculate the retention indices of compounds in the six in-house made mixtures, MegaMix A and MegaMix B.

\subsubsection{Instrumental Data Preprocessing}


LECO ChromaTOF software (version 4.51) equipped with NIST 2014 was used for instrument control as well as for spectrum deconvolution and compound identification. Manufacturer recommended parameters for ChromaTOF were used to reduce the raw instrument data into a list of compounds. These parameters were as follows: baseline offset 0.5 ; auto smoothing; peak width in first dimension $6 \mathrm{~s}$; peak width in the second dimension $0.1 \mathrm{~s}$; signal-to-noise ratio 100 ; match required to combine peaks 600 ; R.T. shift $0.08 \mathrm{~s}$; minimum forward similarity match 600 . The true peak spectrum was also exported as part of the information for each chromatographic peak as absolute intensity values.

GC-MS data was collected by Thermo Xcalibur software (Thermo Fisher Scientific, San Jose, CA, USA) and converted into mzML file format for peak picking and compound identification, using an in-house developed software package. The retention time of each compound generated by in-house software was then converted into a linear retention index in Microsoft Excel (Microsoft, Redmond, WA, USA) using $n$-alkanes homologous series $\left(\mathrm{C}_{7}-\mathrm{C}_{30}\right)$ as reference compounds following the Van Den Doll and Kratz equation. ${ }^{28}$

\subsubsection{Construction of the Second Dimension Retention Index Surface}

We first analyzed the mixture of $\mathrm{C}_{4}-\mathrm{C}_{25}$ FAMEs by GC-MS to get their linear retention index $I_{u}^{T}$ for each compound using $n$-alkanes as references compounds by using Equation 1 described in Chapter 1.

To construct the ${ }^{2} I_{S}, \mathrm{C}_{4}-\mathrm{C}_{25}$ FAMEs were then analyzed by GC×GC-MS in temperature-programmed mode at seventeen ramp rates. After chromatographic peak

picking and compound identification, ${ }^{2} t_{R}$ and ${ }^{2} T_{e}^{\prime}$ were extracted for each FAME compound in each GC×GC-MS analysis. The $I_{u}^{T}$ values calculated from GC-MS data for 
$\mathrm{C}_{4}-\mathrm{C}_{25}$ FAMEs were then used as the second dimension retention indices of $\mathrm{C}_{4}-\mathrm{C}_{25}$ FAMEs. Therefore, a compound $u$ of the $\mathrm{C}_{4}-\mathrm{C}_{25}$ FAMEs was represented by $\left\{{ }^{2} t_{R, u},{ }^{2} T_{e, u}\right.$ , $\left.{ }^{2} I_{u}\right\}$, where ${ }^{2} I_{u}=I_{u}^{T}$. The ${ }^{2} I_{S}$ was then constructed using the data of all $\mathrm{C}_{4}-\mathrm{C}_{25}$ FAMEs, where ${ }^{2} t_{R}$ and ${ }^{2} T_{e}$ formed the $X$ - $Y$ plane and the ${ }^{2} I$ was the $Z$-axis. A polynomial surface model in MATLAB (Mathworks, Natick, MA) named 'polyij' was used to construct the ${ }^{2} I_{S}$, where $i$ was the degree of polynomial in ${ }^{2} t_{R}$, and $j$ was the degree of polynomial in ${ }^{2} T_{e}$. The maximum for both $i$ and $j$ was 5 in the MATLAB platform. The degree of polynomial was the maximum of $i$ and $j$.

\subsubsection{Selection of Optimal Surface Fitting Model}

Multidimensional polynomial model 'polyij' in MATLAB was employed to fit the ${ }^{2} I$ values of all reference compounds $\mathrm{C}_{4}-\mathrm{C}_{25}$ FAMEs into a surface, ${ }^{2} I_{S}$. To prevent overfitting, cross-validation (CV) was employed to select the optimal polynomial surface fitting model. We used $k$-fold cross validation method that partitions data into $k$ randomly chosen subsets (or folds) of roughly equal size. One subset was used to validate the model trained, using the remaining subsets. This process was repeated $k$ times such that each subset was used exactly once for validation. The advantage of this method is that all observations are used for both training and validation, and each observation is used for validation exactly once. The $k$-fold $\mathrm{CV}$ was then repeated $N$ times. In this study, $k$ and $N$ were set to 10 and 100 , respectively.

\subsubsection{Criteria for Evaluating the Surface Fitting}

The criteria for evaluating the quality of surface fitting model were identical to those used in our previous work, including distribution of residuals, R-square $\left(R^{2}\right)$, degrees of 
freedom adjusted R-square $\left(R_{a d j}^{2}\right)$, and root mean squared error $(R M S E){ }^{105}$. Briefly, the distribution of residuals can assess the random errors of surface fitting. $R^{2}$ is the measurement of goodness of fit, which explains the variation of the data. $R_{a d j}^{2}$ adjusts $R^{2}$ based on the residual degrees of freedom, with a value closer to 1 indicating a better fit. RMSE is an estimate of the standard deviation of the random component in the data.

\subsection{Results and Discussion}

A second dimension retention index surface, ${ }^{2} I_{S}$, was created by using the second dimension retention times of $\mathrm{C}_{4}-\mathrm{C}_{25}$ FAMEs acquired at different ramp rates under temperature-programmed mode. All compounds in $\mathrm{C}_{4}-\mathrm{C}_{25}$ FAMEs and compound standards in the six in-house made mixtures were identified from the GCXGC-MS and GCMS data in each chromatographic run, respectively. A total of 102 compounds were identified from the mixtures of MegaMix A and MegaMix B by GC×GC-MS.

\subsubsection{Construction of the Second Dimension Linear Retention Index Surface}

$\mathrm{C}_{4}-\mathrm{C}_{25}$ FAMEs were analyzed in temperature-programmed mode at seventeen different ramp rates $\left({ }^{\circ} \mathrm{C} / \mathrm{min}\right)$. By design, the first dimension column was kept at $285{ }^{\circ} \mathrm{C}$ in every chromatographic run to elute all compounds. Therefore, both the first and the second dimension columns were operated in isothermal mode during the last 13 minutes. Figure 4.1 shows the elution trends of $\mathrm{C}_{4}-\mathrm{C}_{25}$ FAMEs at different ramp rates. A total of 350 data points were acquired under temperature-programmed mode and these data points were used to construct the second dimension retention index surface, ${ }^{2} I_{S}$. 


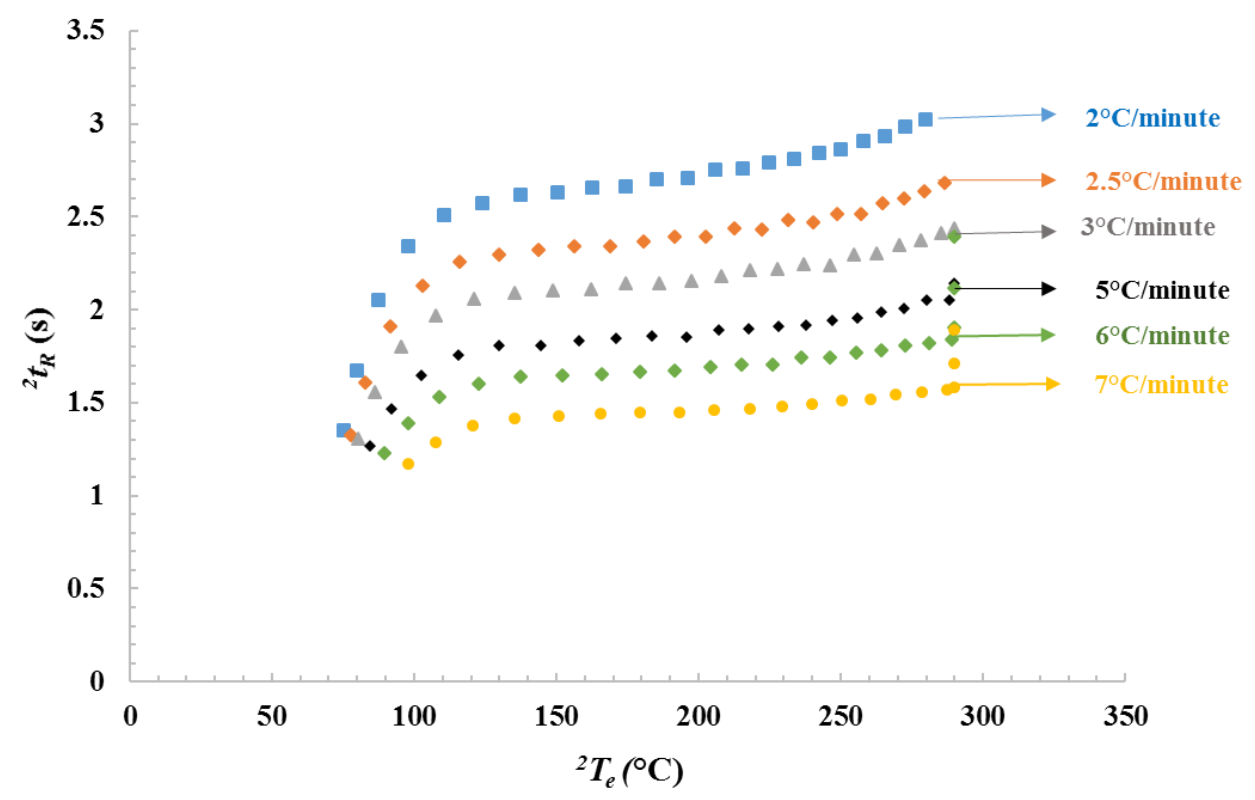

Figure 4.1. The second dimension retention map of C4-C25 FAMEs analyzed in temperature-programmed mode using different ramp rates. Inflection points in the curve indicate the second dimension column temperature was $285{ }^{\circ} \mathrm{C}$, from which the corresponding C4-C25 FAMEs were analyzed in isothermal mode for 13 min.

Multidimensional polynomial model 'polyij' was employed to construct the ${ }^{2} I_{S}$ using those 350 data points. To optimize the $i$ and $j$ values in poly $i j, 10$-fold CV with 100 replications was employed to evaluate the performance of each polynomial model. From those repeated 100 times of the 10 -fold $\mathrm{CV}$, a total of 1000 RMSE values were obtained and their average was denoted as validation RMSE, E. Table 4.1 lists the $E$ values for all possible combinations of $i$ and $j$ in 'poly $i j$ ' $(i=1,2, \ldots, 5 ; j=1,2, \ldots, 5)$. The model 'poly35' $(i=3, j=5)$ has the minimum $E$ value and was selected as the optimal polynomial model to construct ${ }^{2} I_{S}$. 
Table 4.1. Selection of polynomial surface model 'polyij' to construct the second dimension retention index surface using $\mathrm{GC} \times \mathrm{GC}-\mathrm{MS}$ data of $\mathrm{C}_{4}-\mathrm{C}_{25}$ FAMEs

\begin{tabular}{cccccc}
\hline Poly'ij & & $R^{2}$ & $R_{\text {adj }}^{2}$ & $R M S E$ & $E$ \\
\cline { 1 - 3 } $\mathrm{i}$ & $\mathrm{j}$ & & & & \\
\hline 1 & 1 & 0.9760 & 0.9758 & 95.32 & 95.69 \\
2 & 1 & 0.9922 & 0.9921 & 54.57 & 54.87 \\
3 & 1 & 0.9930 & 0.9928 & 51.95 & 52.49 \\
4 & 1 & 0.9933 & 0.9931 & 50.95 & 52.30 \\
5 & 1 & 0.9937 & 0.9935 & 49.25 & 50.55 \\
1 & 2 & 0.9891 & 0.9890 & 64.33 & 64.91 \\
2 & 2 & 0.9988 & 0.9987 & 21.64 & 22.72 \\
3 & 2 & 0.9989 & 0.9988 & 20.78 & 21.26 \\
4 & 2 & 0.9996 & 0.9996 & 12.26 & 20.33 \\
5 & 2 & 0.9996 & 0.9996 & 12.65 & 20.64 \\
1 & 3 & 0.9904 & 0.9902 & 60.67 & 61.74 \\
2 & 3 & 0.9998 & 0.9998 & 8.13 & 9.20 \\
3 & 3 & 0.9999 & 0.9999 & 4.77 & 9.29 \\
4 & 3 & 0.9999 & 0.9999 & 5.83 & 9.70 \\
5 & 3 & 0.9999 & 0.9999 & 6.09 & 9.07 \\
1 & 4 & 0.9910 & 0.9907 & 59.01 & 60.04 \\
2 & 4 & 0.9999 & 0.9999 & 4.93 & 5.08 \\
3 & 4 & 1.0000 & 1.0000 & 2.89 & 4.34 \\
4 & 4 & 1.0000 & 1.0000 & 2.89 & 4.27 \\
5 & 4 & 1.0000 & 1.0000 & 4.05 & 4.10 \\
1 & 5 & 0.9910 & 0.9907 & 59.16 & 60.77 \\
2 & 5 & 0.9999 & 0.9999 & 4.61 & 4.72 \\
$\mathbf{3}$ & $\mathbf{5}$ & $\mathbf{1 . 0 0 0 0}$ & $\mathbf{1 . 0 0 0 0}$ & $\mathbf{3 . 8 3}$ & $\mathbf{3 . 9 1}$ \\
4 & 5 & 1.0000 & 1.0000 & 3.81 & 3.95 \\
5 & 5 & 1.0000 & 1.0000 & 3.84 & 3.96 \\
\hline & & & & &
\end{tabular}

After obtaining the optimal polynomial model 'poly35', all 350 data points of $\mathrm{C}_{4}-\mathrm{C}_{25}$ FAMEs were used to construct ${ }^{2} I_{S}$. The constructed ${ }^{2} I_{S}$ had excellent fitting accuracy with $R^{2}=1.0, R_{a d j}^{2}=1.0$ and $R M S E=3.8$ iu. Among the 100 times of repeated 10 -fold CV, Table 4.2-4.6 lists the results of randomly selected 5 trails of the 10-fold CV. 
Table 4.2. Results of one randomly selected 10-fold cross validation of surface fitting 'poly35' (case one)

\begin{tabular}{ccccc}
\hline \multirow{2}{*}{$\begin{array}{c}\text { Fold } \\
\text { count }\end{array}$} & \multicolumn{2}{c}{ Goodness of fit } & \multicolumn{2}{c}{$\begin{array}{c}\text { Goodness of } \\
\text { validation }\end{array}$} \\
\cline { 2 - 5 } & $R^{2}$ & $R_{\text {adj }}^{2}$ & $R M S E$ & RMSE \\
\hline 1 & 1.0000 & 1.0000 & 3.7 & 4.7 \\
2 & 1.0000 & 1.0000 & 3.9 & 3.5 \\
3 & 1.0000 & 1.0000 & 3.8 & 4.1 \\
4 & 1.0000 & 1.0000 & 3.9 & 3.6 \\
5 & 1.0000 & 1.0000 & 3.8 & 3.9 \\
6 & 1.0000 & 1.0000 & 3.9 & 2.9 \\
7 & 1.0000 & 1.0000 & 3.9 & 3.6 \\
8 & 1.0000 & 1.0000 & 3.8 & 4.5 \\
9 & 1.0000 & 1.0000 & 3.8 & 4.3 \\
10 & 1.0000 & 1.0000 & 3.9 & 3.9 \\
Average & 1.0000 & 1.0000 & 3.8 & 3.9 \\
\hline
\end{tabular}

Table 4.3. Results of one randomly selected 10-fold cross validation of surface fitting 'poly35' (case two)

\begin{tabular}{|c|c|c|c|c|}
\hline \multirow{2}{*}{ Fold count } & \multicolumn{3}{|c|}{ Goodness of fit } & $\begin{array}{l}\text { Goodness of } \\
\text { validation }\end{array}$ \\
\hline & $R^{2}$ & $R_{a d j}^{2}$ & $R M S E$ & $R M S E$ \\
\hline 1 & 1.0000 & 1.0000 & 3.8 & 3.9 \\
\hline 2 & 1.0000 & 1.0000 & 3.8 & 4.0 \\
\hline 3 & 1.0000 & 1.0000 & 3.8 & 3.8 \\
\hline 4 & 1.0000 & 1.0000 & 3.9 & 3.4 \\
\hline 5 & 1.0000 & 1.0000 & 3.9 & 3.4 \\
\hline 6 & 1.0000 & 1.0000 & 3.9 & 3.9 \\
\hline 7 & 1.0000 & 1.0000 & 3.7 & 5.2 \\
\hline 8 & 1.0000 & 1.0000 & 3.9 & 3.3 \\
\hline 9 & 1.0000 & 1.0000 & 3.9 & 3.0 \\
\hline 10 & 1.0000 & 1.0000 & 3.8 & 4.4 \\
\hline Average & 1.0000 & 1.0000 & 3.8 & 3.8 \\
\hline
\end{tabular}


Table 4.4. Results of one randomly selected 10-fold cross validation of surface fitting 'poly35' (case three)

\begin{tabular}{ccccc}
\hline & \multicolumn{2}{c}{ Goodness of fit } & \multicolumn{2}{c}{$\begin{array}{c}\text { Goodness of } \\
\text { validation }\end{array}$} \\
\cline { 2 - 5 } Fold count & $R^{2}$ & $R_{\text {adj }}^{2}$ & $R M S E$ & $R M S E$ \\
\hline 1 & 1.0000 & 1.0000 & 3.8 & 4.4 \\
2 & 1.0000 & 1.0000 & 3.9 & 3.2 \\
3 & 1.0000 & 1.0000 & 3.7 & 4.5 \\
4 & 1.0000 & 1.0000 & 3.8 & 3.7 \\
5 & 1.0000 & 1.0000 & 3.7 & 4.6 \\
6 & 1.0000 & 1.0000 & 3.9 & 3.5 \\
7 & 1.0000 & 1.0000 & 3.9 & 3.0 \\
8 & 1.0000 & 1.0000 & 3.9 & 3.9 \\
9 & 1.0000 & 1.0000 & 3.8 & 4.1 \\
10 & 1.0000 & 1.0000 & 3.9 & 3.9 \\
Average & 1.0000 & 1.0000 & 3.8 & 3.9 \\
\hline
\end{tabular}

Table 4.5. Results of one randomly selected 10-fold cross validation of surface fitting 'poly35' (case four)

\begin{tabular}{ccccc}
\hline & \multicolumn{2}{c}{ Goodness of fit } & \multicolumn{2}{c}{$\begin{array}{c}\text { Goodness of } \\
\text { validation }\end{array}$} \\
\cline { 2 - 5 } $\begin{array}{c}\text { Fold } \\
\text { count }\end{array}$ & $R^{2}$ & $R_{a d j}^{2}$ & $R M S E$ & $R M S E$ \\
\hline 1 & 1.0000 & 1.0000 & 3.8 & 4.4 \\
2 & 1.0000 & 1.0000 & 3.9 & 3.8 \\
3 & 1.0000 & 1.0000 & 3.8 & 3.9 \\
4 & 1.0000 & 1.0000 & 3.7 & 4.5 \\
5 & 1.0000 & 1.0000 & 3.9 & 3.5 \\
6 & 1.0000 & 1.0000 & 3.9 & 3.2 \\
7 & 1.0000 & 1.0000 & 3.7 & 5.3 \\
8 & 1.0000 & 1.0000 & 3.9 & 3.1 \\
9 & 1.0000 & 1.0000 & 3.9 & 3.0 \\
10 & 1.0000 & 1.0000 & 3.8 & 3.8 \\
Average & 1.0000 & 1.0000 & 3.8 & 3.8 \\
\hline
\end{tabular}


Table 4.6. Results of one randomly selected 10-fold cross validation of surface fitting 'poly35' (case five)

\begin{tabular}{|c|c|c|c|c|}
\hline \multirow[b]{2}{*}{$\begin{array}{l}\text { Fold } \\
\text { count }\end{array}$} & \multicolumn{3}{|c|}{ Goodness of fit } & \multirow{2}{*}{$\begin{array}{l}\text { Goodness of } \\
\text { validation } \\
R M S E\end{array}$} \\
\hline & $\overline{R^{2}}$ & $R_{a d j}^{2}$ & $R M S E$ & \\
\hline 1 & 1.0000 & 1.0000 & 3.9 & 3.5 \\
\hline 2 & 1.0000 & 1.0000 & 3.8 & 4.3 \\
\hline 3 & 1.0000 & 1.0000 & 3.9 & 3.6 \\
\hline 4 & 1.0000 & 1.0000 & 3.8 & 4.2 \\
\hline 5 & 1.0000 & 1.0000 & 3.9 & 3.5 \\
\hline 6 & 1.0000 & 1.0000 & 3.7 & 4.7 \\
\hline 7 & 1.0000 & 1.0000 & 3.9 & 3.1 \\
\hline 8 & 1.0000 & 1.0000 & 3.8 & 3.7 \\
\hline 9 & 1.0000 & 1.0000 & 3.8 & 4.1 \\
\hline 10 & 1.0000 & 1.0000 & 3.8 & 4.2 \\
\hline Average & 1.0000 & 1.0000 & 3.8 & 3.9 \\
\hline
\end{tabular}

The very close results of these trials demonstrate that the polynomial surface model 'poly35' was robust in terms of prediction. The $S T D$ of the 1000 (i.e., $100 \times 10$ ) validations RMSE was $0.49 \mathrm{iu}$, also demonstrating that the 'poly35' model is robust. Figure 4.2(A) depicts the ${ }^{2} I_{S}$ constructed from the temperature-programmed data of the $\mathrm{C}_{4}-\mathrm{C}_{25}$ FAMEs using polynomial surface fitting model 'poly35'. Figure 4.2(B) is the residual plot of the surface fitting. The residuals are symmetrically distributed Figure 4.2(C) around the fitted plane suggesting that its variance is constant and there was no bias introduced by the polynomial surface model 'poly35'. The residuals were in a range of 0-14 iu with an average value of $2.8 \mathrm{iu}$. 
A.

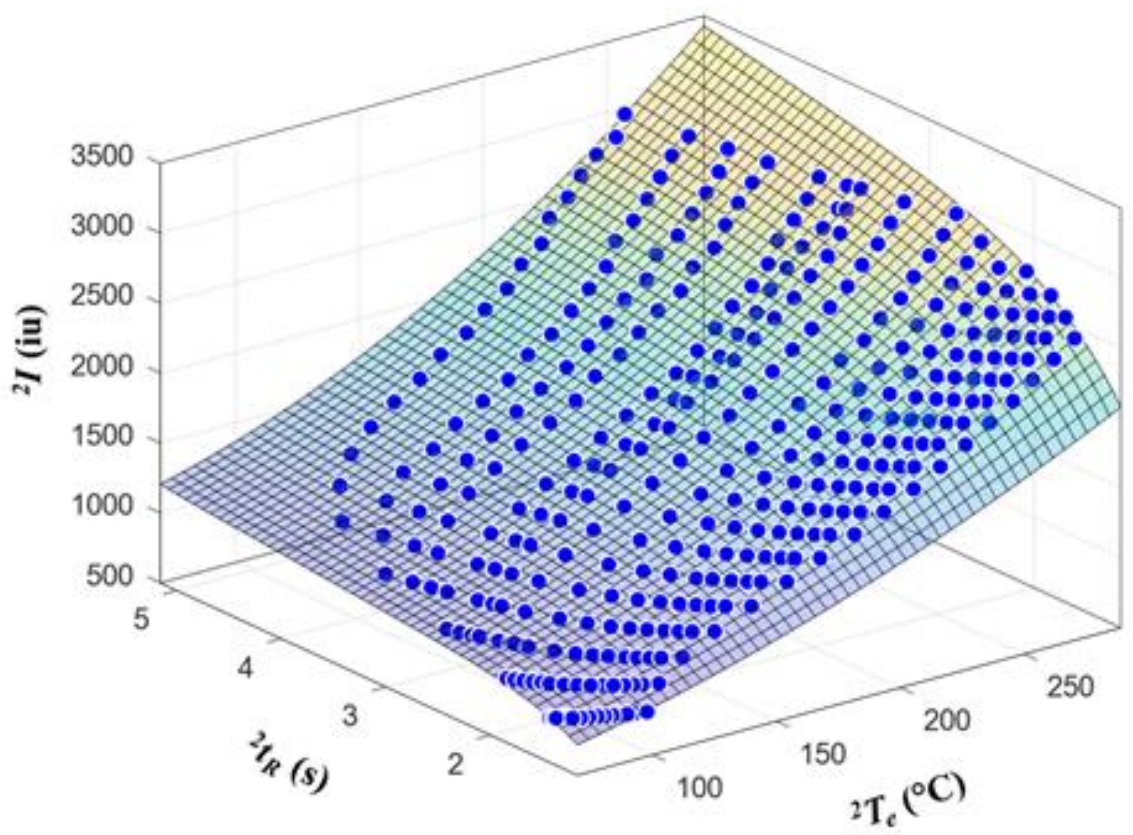

B.

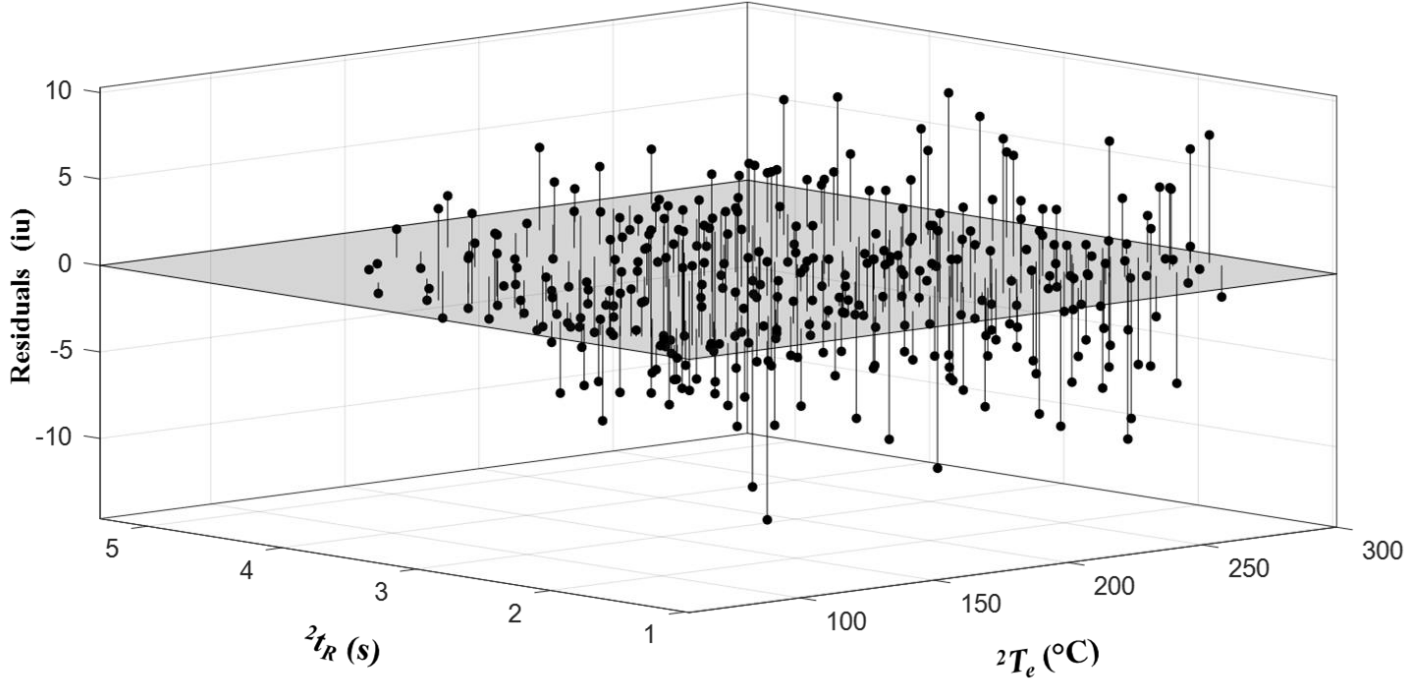


C.

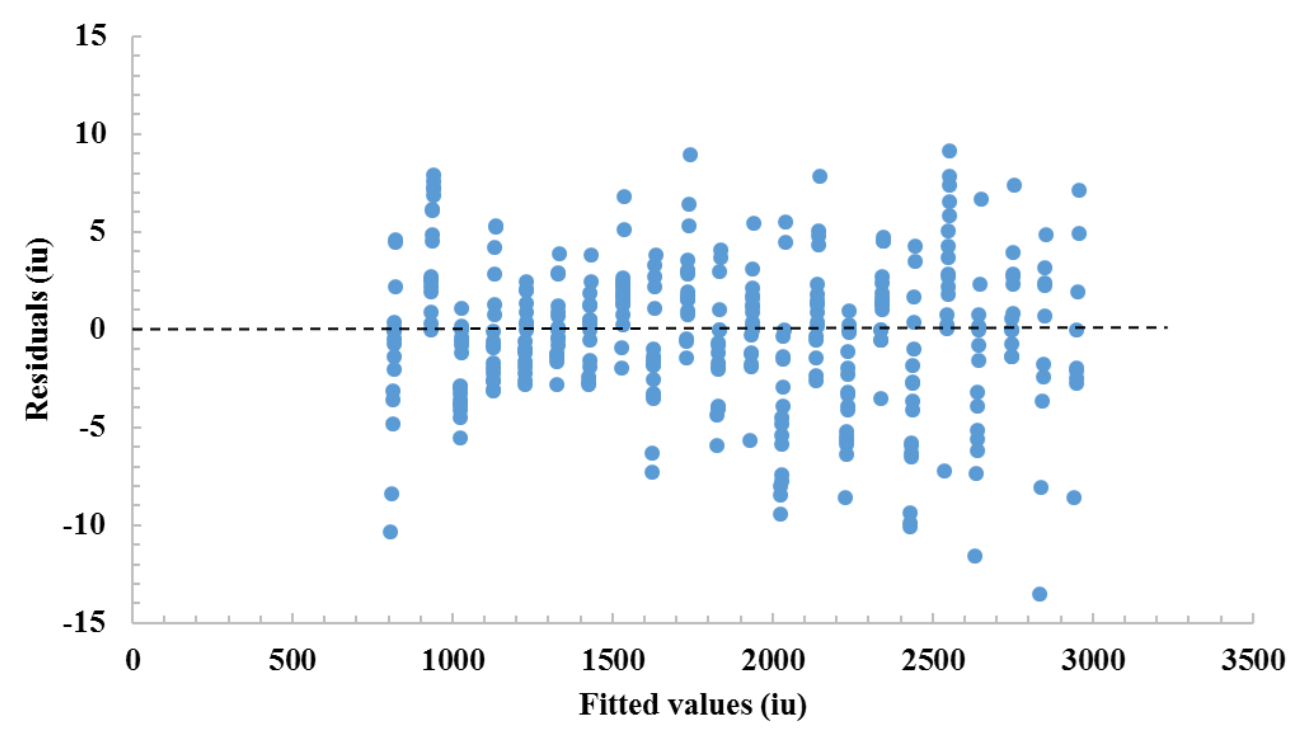

Figure 4.2. Second dimension retention index surface constructed using the GC $\times$ GC-MS data of $\mathrm{C}_{4}-\mathrm{C}_{25} \mathrm{FAMEs}$. The retention index surface is a function of the elution temperature of each $\mathrm{C}_{4}-\mathrm{C}_{25}$ FAMEs in the second dimension column ${ }^{2} T_{e}$, and the corresponding unadjusted retention time ${ }^{2} t_{R}$. (A) Is the second dimension retention index surface, (B) is the residual plot and $(\mathbf{C})$ is the distribution of the residuals.

Figure 4.3 shows the second dimension retention maps constructed from $\mathrm{C}_{4}-\mathrm{C}_{25}$ FAMEs and $n$-alkanes data, respectively. $\mathrm{C}_{4}-\mathrm{C}_{25}$ FAMEs data demonstrated greatly increased coverage of the second dimension separation space compared to the separation space covered by the $n$-alkanes data. 


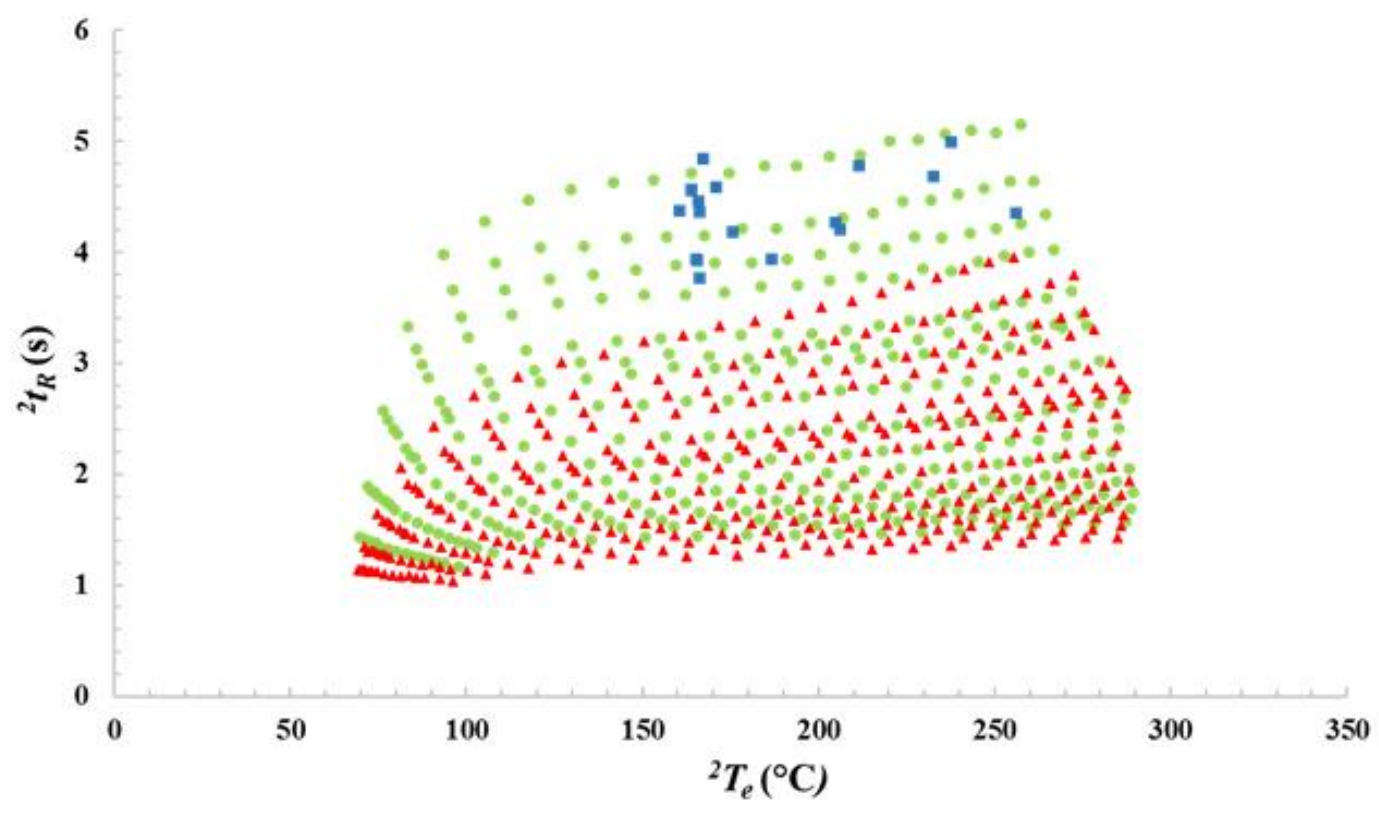

Figure 4.3. Second dimension retention map constructed from $\mathrm{C}_{4}-\mathrm{C}_{25}$ FAMEs and $n$ alkanes data. Red triangles are the data points of $n$-alkanes. Green circles are the data points of C4- C25 FAMEs. Blue rectangles are the data points of compounds in MegaMix A.

All compounds in MegaMix A, which were eluted beyond the retention map of $n$ alkanes, but were within the retention map of $\mathrm{C}_{4}-\mathrm{C}_{25}$ FAMEs. Encompassing the ${ }^{2} I$ of all compounds of MegaMix A in the retention map ensures that the ${ }^{2} I$ of those compounds can be directly calculated from the ${ }^{2} I_{S}$ without extrapolation.

\subsubsection{The Second Dimension Retention Indices of $n$-Alkanes}

$n$-Alkanes are the most widely used reference series in calculating retention index. In this study, we used $\mathrm{C}_{4}-\mathrm{C}_{25}$ FAMEs as a reference series to construct the ${ }^{2} I_{S}$, where the Zaxis was the $I^{T}$ values of $\mathrm{C}_{4}-\mathrm{C}_{25}$ FAMEs that were calculated from GC-MS data using $n$ - 
alkane as references. Therefore, the ${ }^{2} I_{S}$ of $\mathrm{C}_{4}-\mathrm{C}_{25}$ FAMEs are in $n$-alkane scale. We analyzed $n$-alkanes by GC×GC-MS in seventeen different ramp rates ranged from a very slow ramp rate of $0.85^{\circ} \mathrm{C} / \mathrm{min}$ to a fast ramp rate of $7.0^{\circ} \mathrm{C} / \mathrm{min}$. A total of 364 data points were acquired for $n$-alkanes. We then calculated ${ }^{2} I$ values of these 364 data points from the ${ }^{2} I_{S}$ constructed using the $I^{T}$ values of $\mathrm{C}_{4}-\mathrm{C}_{25}$ FAMEs. Figure 4.4 shows that the STD of the calculated ${ }^{2} I$ of the $n$-alkanes among the seventeen chromatographic runs ranged from 3.6 iu to $14.0 \mathrm{iu}$, demonstrating that the developed method had an excellent reproducibility in calculating the ${ }^{2} I$ of $n$-alkanes.

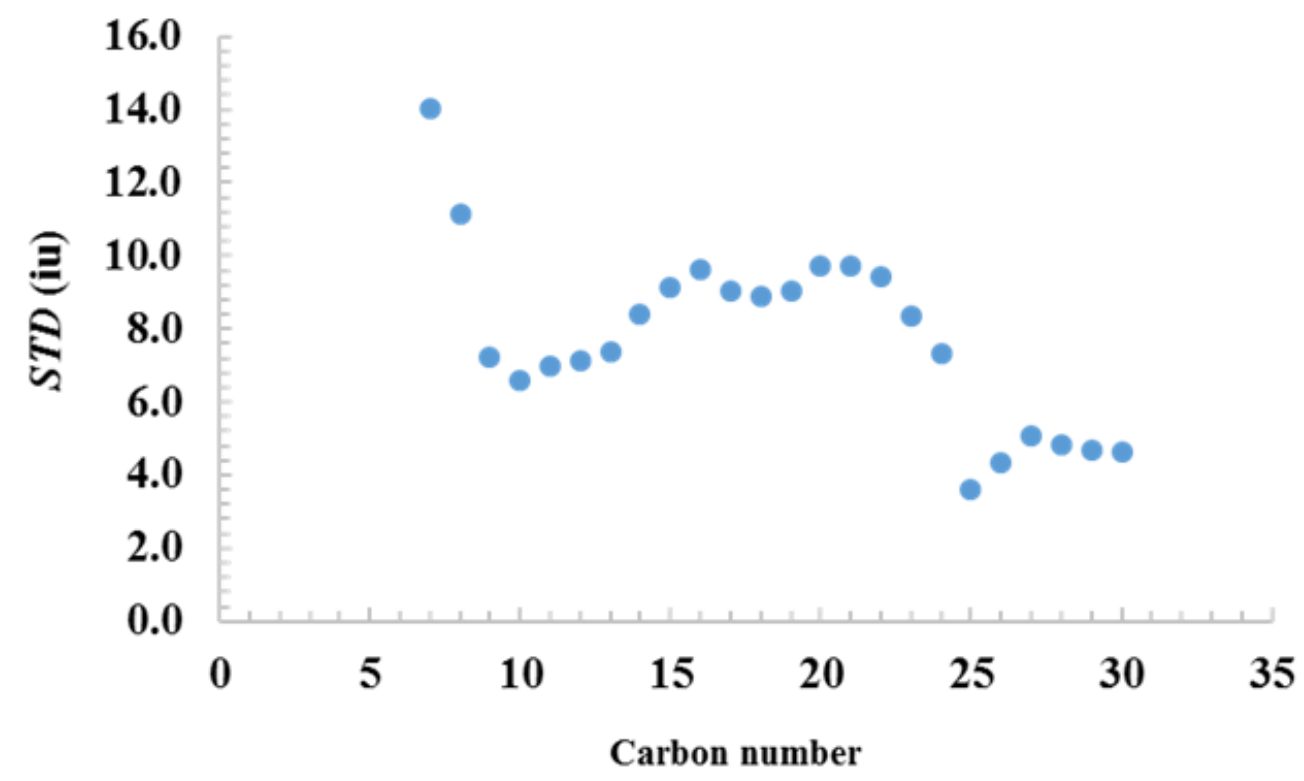

Figure 4.4. Standard deviation of calculated ${ }^{2} I$ of each $n$-alkane compound among the seventeen chromatographic runs with different ramp rates. 
The calculated ${ }^{2} I$ values of $n$-alkanes also showed an excellent agreement with their hypothetical retention index, i.e., $100 \times n$. Figure 4.5 depicts the distribution of the variation of the calculated ${ }^{2} I$ of the $n$-alkanes from their hypothetical values. The variation ranged from $0.0 \mathrm{iu}$ to $50 \mathrm{iu}$ with an average variation of $11.8 \mathrm{iu}$. Among the 364 data points of $n$-alkanes, $94 \%$ of data points were within 20 iu variation. The deviation of $\mathrm{C}_{7}$-alkane was $50 \mathrm{iu}$, and that was the largest value.

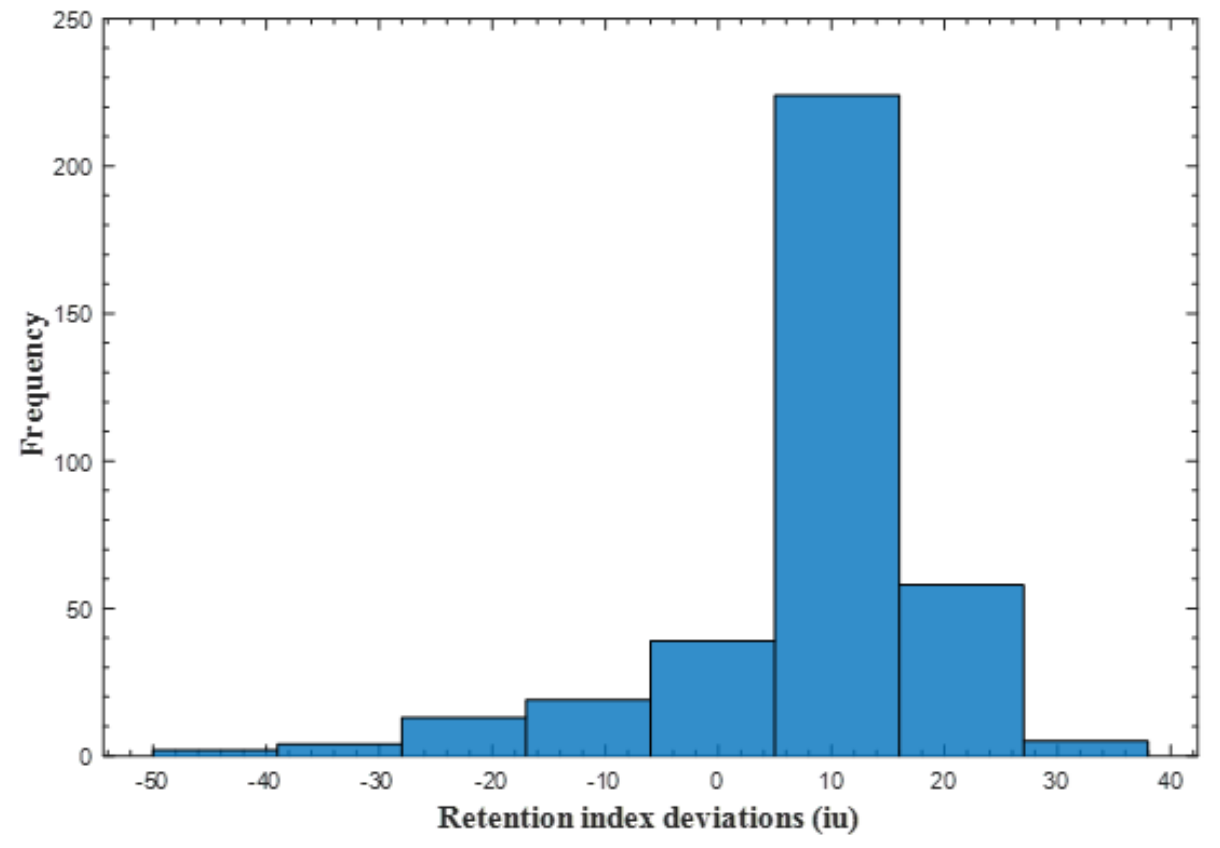

Figure 4.5. Retention index variation of $n$-alkanes between the calculated ${ }^{2} I$ from their hypothetical value $100 \times n$. $n$ is the number of carbon atoms in an $n$-alkane. 
Figure 4.6 shows that all $n$-alkanes, except $\mathrm{C}_{7}$-alkane, had near exponential decrease of ${ }^{2} t_{R}$ with the increase of ${ }^{2} T_{e}$. This exceptional relation between temperature and retention time might contribute to the high variation of $\mathrm{C}_{7}$-alkane. The observed variation could also be related to temperature dependency of the calculated retention index value $78,79$.

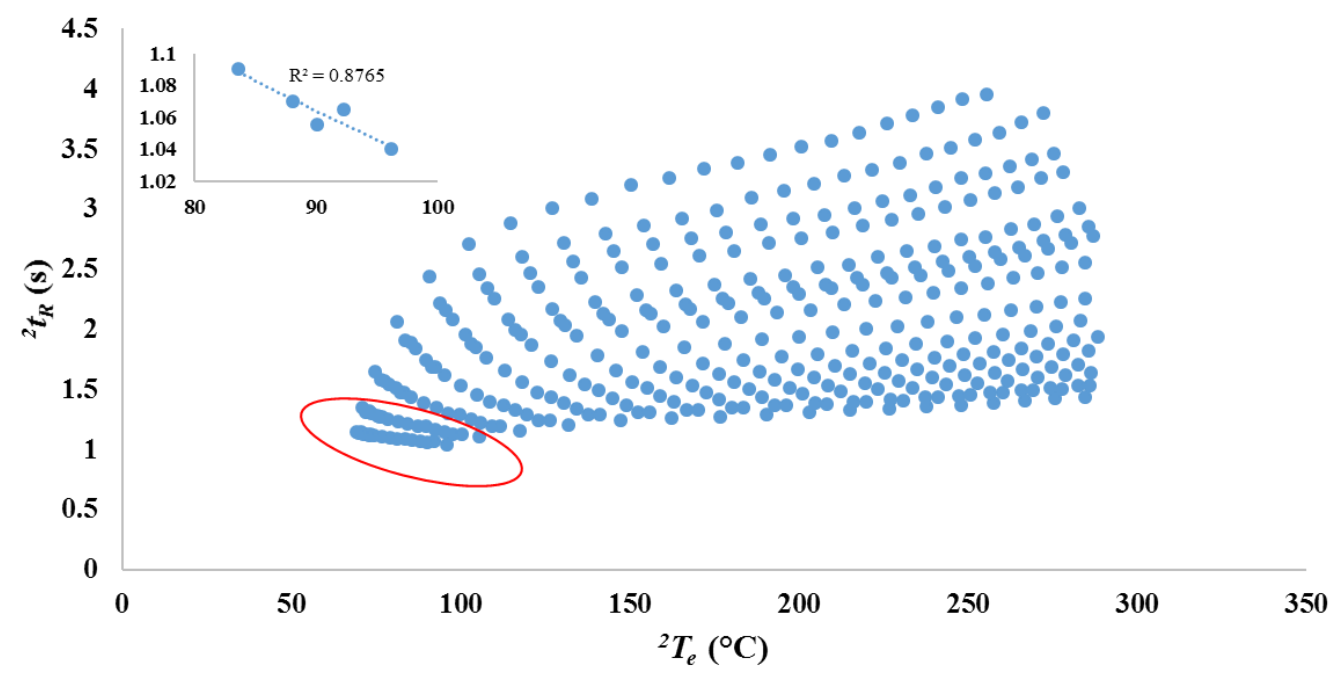

Figure 4.6. The second dimension retention map of n-alkanes. The inset shows the relation between ${ }^{2} T_{e}$ and ${ }^{2} t_{R}$ for $\mathrm{C}_{7}$-alkane.

A previous study of the NIST retention index database revealed that the retention index deviation is column dependent, and the variation of linear retention index increases with the increase of column polarity. ${ }^{106}$ The NIST retention index database registered three types of columns, i.e., standard non-polar, semi non-polar and standard polar. The polarity of the DB-17ms column used in this study is between the standard polar column and the standard non-polar column. 
The ${ }^{2} I$ variations of the $n$-alkanes, i.e., $11.8 \mathrm{iu}$, is much smaller than the variation reported in the literature concerning the NIST retention index database, demonstrating that the method developed in this work introduces much smaller variation in calculating the linear retention index for compounds separated on the second dimension column of GC $\times$ GC-MS. Therefore, the ${ }^{2}$ Ivalues calculated from the developed method can be used to aid compound identification in analysis of GC×GC-MS data.

\subsubsection{Calculating the ${ }^{2} I$ of Compound Standards}

The six in-house made mixtures, MegaMix A and MegaMix B were analyzed in temperature-programmed mode by GC×GC-MS. Table 4.7 lists the calculated ${ }^{2} I$ values of the 80 compound standards using their second dimension retention times and the ${ }^{2} I_{S}$ constructed in Section 3.1.

Tables 4.8 and 4.9 list the calculated ${ }^{2}$ I values of compounds in MegaMix A and MegaMix B, respectively. In order to validate the ${ }^{2} I$ values calculated by the method developed in this study, we analyzed the 80 compound standards, MegaMix A and MegaMix B by GCMS in temperature-programmed mod 
Table 4.7. Second dimension retention indices ${ }^{2} I$ of the 80 compound standards

\begin{tabular}{|c|c|c|c|c|c|c|c|c|c|}
\hline \multirow{2}{*}{$\begin{array}{l}\text { Compounds name } \\
\text { Ramp rate }\left({ }^{\circ} \mathrm{C} / \mathrm{min}\right)\end{array}$} & \multicolumn{3}{|c|}{$I^{2}(\mathrm{iu})^{\mathrm{a}}$} & & & \multirow{2}{*}{$\begin{array}{l}\text { STD } \\
\text { (iu) }\end{array}$} & \multirow{2}{*}{$\begin{array}{l}\text { RSD } \\
(\%)^{\mathrm{b}}\end{array}$} & \multirow{2}{*}{$\begin{array}{l}{ }^{1} I \text { from } \\
\text { GC-MSc }\end{array}$} & \multirow{2}{*}{$\Delta^{2} \mathrm{~T}_{\mathrm{e}}^{\mathrm{d}}$} \\
\hline & 4 & 4.5 & 5 & 5.5 & 6 & & & & \\
\hline \multicolumn{10}{|l|}{ FAME } \\
\hline Methyl butyrate & 818 & 818 & 819 & 820 & 826 & 3 & 0.33 & & 8.9 \\
\hline Methyl valerate & 920 & 923 & 928 & 923 & 924 & 3 & 0.27 & & 10.5 \\
\hline Methyl caproate & 1029 & 1028 & 1029 & 1030 & 1030 & 1 & 0.06 & 1028 & 12.7 \\
\hline Methyl enanthate & 1126 & 1127 & 1127 & 1127 & 1125 & 1 & 0.06 & 1129 & 13.9 \\
\hline Methyl caprylate & 1228 & 1226 & 1228 & 1227 & 1225 & 1 & 0.09 & 1229 & 14.9 \\
\hline Methyl pelargonate & 1324 & 1322 & 1327 & 1325 & 1323 & 2 & 0.14 & 1329 & 14.7 \\
\hline Methyl caprate & 1425 & 1425 & 1428 & 1427 & 1423 & 2 & 0.11 & 1429 & 15 \\
\hline Methyl undecanoate & 1522 & 1521 & 1530 & 1528 & 1521 & 4 & 0.24 & 1531 & 15.2 \\
\hline Methyl laurate & 1623 & 1623 & 1630 & 1624 & 1624 & 3 & 0.17 & 1630 & 15.4 \\
\hline Methyl tridecanoate & 1722 & 1723 & 1733 & 1725 & 1717 & 5 & 0.29 & 1733 & 15.4 \\
\hline Methyl myristate & 1823 & 1822 & 1835 & 1827 & 1824 & 5 & 0.26 & 1832 & 15.5 \\
\hline Methyl pentadecanoate & 1922 & 1924 & 1933 & 1929 & 1921 & 5 & 0.24 & 1935 & 15.4 \\
\hline Methyl palmitate & 2029 & 2026 & 2037 & 2031 & 2018 & 6 & 0.31 & 2034 & 15.9 \\
\hline Methyl margarate & 2125 & 2120 & 2138 & 2126 & 2117 & 7 & 0.33 & 2138 & 15.9 \\
\hline Methyl stearate & 2227 & 2228 & 2244 & 2230 & 2223 & 7 & 0.32 & 2237 & 15.5 \\
\hline Methyl nonadecanoate & 2323 & 2323 & 2335 & 2328 & 2318 & 6 & 0.24 & 2340 & 15.9 \\
\hline Methyl arachidate & 2427 & 2427 & 2439 & 2432 & 2419 & 7 & 0.27 & 2440 & 15.7 \\
\hline Methyl heneicosanoate & 2523 & 2525 & 2538 & 2533 & 2519 & 7 & 0.27 & 2545 & 15.9 \\
\hline Methyl behenate & 2629 & 2629 & 2641 & & & 5 & 0.21 & 2644 & \\
\hline Methyl tricosanoate & 2726 & & & & & 0 & 0 & & \\
\hline \multicolumn{10}{|l|}{ Fatty acid } \\
\hline Butyric acid & 890 & 899 & 897 & 888 & 892 & 4 & 0.47 & & 10 \\
\hline Valeric acid & 990 & 1003 & 996 & 991 & 994 & 4 & 0.45 & & 12.2 \\
\hline Caproic acid & 1092 & 1105 & 1099 & 1090 & 1092 & 5 & 0.49 & 1113 & 13.7 \\
\hline
\end{tabular}




\begin{tabular}{|c|c|c|c|c|c|c|c|c|c|}
\hline Enanthic acid & 1188 & 1197 & 1200 & 1190 & 1190 & 5 & 0.4 & 1210 & 14.5 \\
\hline Caprylic acid & 1290 & 1301 & 1285 & 1294 & 1290 & 5 & 0.4 & 1304 & 14.9 \\
\hline Pelargonic acid & 1393 & 1406 & 1404 & 1395 & 1397 & 5 & 0.36 & 1407 & 14.7 \\
\hline Capric acid & 1491 & 1509 & 1508 & 1495 & 1498 & 7 & 0.48 & 1501 & 14.5 \\
\hline Undecylic acid & 1591 & 1611 & 1608 & 1602 & 1606 & 7 & 0.42 & 1598 & 15.2 \\
\hline Lauric acid & 1693 & 1718 & 1709 & 1699 & 1708 & 9 & 0.52 & 1697 & 14.9 \\
\hline Tridecylic acid & 1798 & 1814 & 1812 & 1804 & 1807 & 6 & 0.31 & 1798 & 15 \\
\hline Myristic acid & 1906 & 1929 & 1913 & 1916 & 1915 & 7 & 0.39 & 1901 & 15.9 \\
\hline Pentadecylic acid & 2000 & 2018 & 2007 & 2005 & 2010 & 6 & 0.29 & 1999 & 15.7 \\
\hline Palmitic acid & 2104 & 2117 & 2113 & 2113 & 2112 & 4 & 0.21 & 2101 & 15.7 \\
\hline Margaric acid & 2204 & 2219 & 2215 & 2218 & 2215 & 5 & 0.24 & 2205 & 15.5 \\
\hline Stearic acid & 2309 & 2325 & 2313 & 2313 & 2313 & 5 & 0.23 & 2307 & 16 \\
\hline Nonadecylic acid & 2412 & 2419 & 2418 & 2412 & 2413 & 3 & 0.13 & 2409 & 15.5 \\
\hline Arachidic acid & 2512 & 2519 & 2516 & 2515 & 2515 & 2 & 0.09 & 2513 & 15.9 \\
\hline Heneicosylic acid & 2614 & 2627 & 2618 & 2621 & & 5 & 0.18 & 2618 & \\
\hline Behenic acid & 2724 & 2724 & & & & 0 & 0 & & \\
\hline \multicolumn{10}{|l|}{ Ketone } \\
\hline 2-Pentanone & 805 & 808 & 802 & 815 & 800 & 5 & 0.66 & & 8.5 \\
\hline 2-Hexanone & 909 & 899 & 907 & 914 & 907 & 5 & 0.55 & & 10 \\
\hline 2-Heptanone & 1013 & 1012 & 1009 & 1015 & 1012 & 2 & 0.19 & 1008 & 12.2 \\
\hline 2-Octanone & 1116 & 1114 & 1111 & 1115 & 1110 & 2 & 0.2 & 1109 & 13.5 \\
\hline 2-Nonanone & 1216 & 1218 & 1209 & 1215 & 1212 & 3 & 0.25 & 1208 & 14.2 \\
\hline 2-Decanone & 1314 & 1316 & 1309 & 1316 & 1312 & 3 & 0.22 & 1308 & 14.9 \\
\hline 2-Undecanone & 1414 & 1418 & 1410 & 1419 & 1411 & 4 & 0.25 & 1410 & 15 \\
\hline 2-Dodecanone & 1517 & 1518 & 1511 & 1527 & 1514 & 6 & 0.36 & 1512 & 15.4 \\
\hline 2-Tridecanone & 1619 & 1617 & 1610 & 1620 & 1613 & 4 & 0.22 & 1612 & 15.7 \\
\hline 2-Tetradecanone & 1718 & 1718 & 1711 & 1724 & 1714 & 5 & 0.27 & 1714 & 15.4 \\
\hline 2-Pentadecanone & 1823 & 1824 & 1813 & 1822 & 1814 & 5 & 0.27 & 1816 & 16 \\
\hline 2-Hexadecanone & 1927 & 1927 & 1918 & 1934 & 1917 & 6 & 0.33 & 1918 & 16 \\
\hline 2-Heptadecanone & 2015 & 2015 & 2014 & 2020 & 2015 & 2 & 0.1 & 2017 & 15.9 \\
\hline 2-Octadecanone & 2126 & 2135 & 2120 & 2137 & 2120 & 7 & 0.35 & 2123 & 15.9 \\
\hline
\end{tabular}




\begin{tabular}{|c|c|c|c|c|c|c|c|c|c|}
\hline 2-Nonadecanoe & 2233 & 2234 & 2222 & 2240 & 2227 & 6 & 0.29 & 2225 & 16 \\
\hline \multicolumn{10}{|l|}{ Alcohol } \\
\hline 1-Pentanol & 863 & 868 & 866 & 870 & 871 & 3 & 0.3 & & 9.7 \\
\hline 1-Hexanol & 966 & 974 & 970 & 969 & 971 & 2 & 0.25 & & 11.7 \\
\hline 1-Heptanol & 1068 & 1070 & 1068 & 1071 & 1068 & 1 & 0.11 & 1069 & 13.4 \\
\hline 1-Octanol & 1172 & 1175 & 1171 & 1172 & 1170 & 1 & 0.12 & 1174 & 14.2 \\
\hline 1-Nonanol & 1273 & 1276 & 1262 & 1268 & 1266 & 5 & 0.39 & 1275 & 20 \\
\hline 1-Undecanol & 1471 & 1475 & 1463 & 1471 & 1469 & 4 & 0.27 & 1480 & 17.7 \\
\hline 1-Dodecanol & 1567 & 1576 & 1564 & 1570 & 1570 & 4 & 0.26 & 1582 & 14.5 \\
\hline \multicolumn{10}{|l|}{ Iodobutane } \\
\hline 1-Iodobutane & 939 & 944 & 937 & 943 & 944 & 3 & 0.3 & & 11 \\
\hline 1-Iodopentane & 1052 & 1053 & 1054 & 1055 & 1058 & 2 & 0.18 & 1065 & 13 \\
\hline 1-Iodohexane & 1159 & 1161 & 1161 & 1162 & 1162 & 1 & 0.1 & 1168 & 14 \\
\hline 1-Iodoheptane & 1265 & 1269 & 1268 & 1269 & 1269 & 1 & 0.11 & 1273 & 14.9 \\
\hline 1-Iodooctane & 1373 & 1374 & 1375 & 1380 & 1378 & 3 & 0.2 & 1380 & 15.4 \\
\hline 1-Iodononane & 1480 & 1483 & 1481 & 1485 & 1491 & 4 & 0.27 & 1488 & 15.7 \\
\hline 1-Iododecane & 1594 & 1597 & 1588 & 1595 & 1591 & 3 & 0.19 & 1596 & 16 \\
\hline 1-Iodoundecane & 1695 & 1698 & 1696 & 1702 & 1701 & 3 & 0.16 & 1702 & 16 \\
\hline 1-Iodododecane & 1804 & 1807 & 1803 & 1810 & 1811 & 3 & 0.17 & 1810 & 15.7 \\
\hline 1-Iodohexadecane & 2234 & 2235 & 2233 & 2243 & 2236 & 3 & 0.15 & 2237 & 16.2 \\
\hline 1-Iodooctadecane & 2445 & 2451 & 2446 & 2457 & 2450 & 4 & 0.17 & 2449 & 16.4 \\
\hline \multicolumn{10}{|l|}{ Aldehyde } \\
\hline Pentanal & 808 & 807 & 807 & 814 & 812 & 3 & 0.36 & & 8.4 \\
\hline Hexanal & 909 & 909 & 911 & 913 & 912 & 2 & 0.17 & & 10.5 \\
\hline Heptanal & 1018 & 1016 & 1015 & 1019 & 1017 & 1 & 0.14 & 1016 & 12.5 \\
\hline Octanal & 1121 & 1120 & 1117 & 1121 & 1119 & 1 & 0.13 & 1119 & 14 \\
\hline Nonanal & 1221 & 1219 & 1219 & 1221 & 1220 & 1 & 0.07 & 1220 & 14.7 \\
\hline
\end{tabular}


Table 4.8. The second dimension linear retention indices ${ }^{2} I$ of compounds identified from MegaMix A

\begin{tabular}{|c|c|c|c|c|c|c|c|c|c|c|}
\hline Chromatographic conditions & & & & $\begin{array}{l}2 \mathrm{I} \\
\mathrm{u})^{\mathrm{a}}\end{array}$ & & & & & $\begin{array}{l}R S D \\
(\%)^{\mathrm{b}}\end{array}$ & $\begin{array}{c}\Delta^{2} T_{e} \\
\left({ }^{\circ} \mathrm{C}\right)^{\mathrm{c}}\end{array}$ \\
\hline Secondary oven offset $\left({ }^{\circ} \mathrm{C}\right)$ & +5 & +5 & +5 & +5 & +5 & +5 & +5 & +10 & & \\
\hline Ramp rate $\left({ }^{\circ} \mathrm{C} /\right.$ minute $)$ & 4 & 4.5 & 5 & 5 & 5.5 & 5.5 & 6 & 5 & & \\
\hline Modulation period (s) & 5 & 5 & 5 & 4 & 5 & 4 & 5 & 4 & & \\
\hline N-Nitrosodimethylamine & 959 & 959 & 959 & 967 & 961 & 961 & 959 & 960 & 0.26 & 9.5 \\
\hline Aniline & 1220 & 1225 & 1229 & 1235 & 1232 & 1232 & 1235 & 1235 & 0.41 & 13.9 \\
\hline Phenol & 1173 & 1176 & 1177 & 1186 & 1180 & 1179 & 1180 & 1182 & 0.32 & 13.4 \\
\hline Bis(2-chloroethyl) ether & 1189 & 1191 & 1193 & 1200 & 1194 & 1194 & 1194 & 1196 & 0.26 & 13.4 \\
\hline 2-Chlorophenol & 1183 & 1187 & 1189 & 1198 & 1194 & 1191 & 1192 & 1195 & 0.38 & 14 \\
\hline Benzyl alcohol & 1264 & 1269 & 1271 & 1280 & 1277 & 1275 & 1277 & 1279 & 0.39 & 14.4 \\
\hline $\begin{array}{l}\text { 2,2'-Oxybis }(2- \\
\text { chloropropane })\end{array}$ & 1215 & 1216 & 1216 & 1225 & 1218 & 1215 & 1217 & 1218 & 0.24 & 14.5 \\
\hline 2-Methylphenol & 1257 & 1260 & 1262 & 1270 & 1266 & 1263 & 1265 & 1268 & 0.32 & 14.5 \\
\hline N-nitrosodipropylamine & 1271 & 1272 & 1275 & 1284 & 1277 & 1276 & 1276 & 1279 & 0.3 & 14.7 \\
\hline 3-Methylphenol & 1278 & 1282 & 1282 & 1292 & 1286 & 1285 & 1286 & 1289 & 0.33 & 14.2 \\
\hline Hexachloroethane & 1242 & 1244 & 1247 & 1252 & 1247 & 1247 & 1251 & 1248 & 0.25 & 15 \\
\hline Isophorone & 1338 & 1342 & 1344 & 1353 & 1347 & 1347 & 1348 & 1351 & 0.33 & 14.7 \\
\hline 2-Nitrophenol & 1382 & 1386 & 1391 & 1399 & 1397 & 1397 & 1401 & 1400 & 0.46 & 15 \\
\hline $\begin{array}{l}\text { 2,4-Dimethylphenol } \\
\text { Bis(2-chloroethoxy)- }\end{array}$ & 1360 & 1362 & 1364 & 1375 & 1369 & 1367 & 1369 & 1372 & 0.36 & 15 \\
\hline Methane & 1387 & 1390 & 1392 & 1401 & 1397 & 1395 & 1396 & 1399 & 0.32 & 14.7 \\
\hline 2,4-DichloroPhenol & 1394 & 1398 & 1401 & 1412 & 1408 & 1408 & 1409 & 1411 & 0.44 & 14.2 \\
\hline 4-Chloroaniline & 1498 & 1506 & 1512 & 1520 & 1519 & 1519 & 1523 & 1522 & 0.54 & 15.2 \\
\hline 4-Chloro-3-methylphenol & 1543 & 1549 & 1554 & 1563 & 1559 & 1558 & 1562 & 1563 & 0.44 & 15.7 \\
\hline 2-Methylnaphthalene & 1561 & 1569 & 1575 & 1585 & 1582 & 1581 & 1585 & 1586 & 0.53 & 15.9 \\
\hline 1-Methylnaphthalene & 1594 & 1602 & 1610 & 1617 & 1618 & 1616 & 1620 & 1620 & 0.55 & 16.4 \\
\hline
\end{tabular}




\begin{tabular}{|c|c|c|c|c|c|c|c|c|c|c|}
\hline $\begin{array}{l}\text { 1,3-Cyclopentadiene, } \\
1,2,3,4,5,5 \text {-hexachloro- }\end{array}$ & 1523 & 1529 & 1533 & 1544 & 1537 & 1536 & 1537 & 1542 & 0.41 & 16.2 \\
\hline 2,4,6-Trichlorophenol & 1616 & 1624 & 1628 & 1640 & 1634 & 1635 & 1640 & 1647 & 0.56 & 16 \\
\hline 2,4,5-Trichlorophenol & 1623 & 1630 & 1635 & 1647 & 1644 & 1641 & 1647 & 1647 & 0.51 & 16.2 \\
\hline 2-Chloronaphthalene & 1676 & 1684 & 1692 & 1701 & 1701 & 1701 & 1703 & 1704 & 0.57 & 16.4 \\
\hline 2-Nitroaniline & 1793 & 1801 & 1809 & 1815 & 1819 & 1818 & 1824 & 1820 & 0.55 & 16 \\
\hline 1,4-Dinitrobenzene & 1836 & 1840 & 1848 & 1855 & 1858 & 1856 & 1862 & 1858 & 0.48 & 15.7 \\
\hline 1,3-Dinitrobenzene & 1846 & 1852 & 1859 & 1866 & 1868 & 1868 & 1874 & 1868 & 0.48 & 16 \\
\hline Dimethyl phthalate & 1798 & 1805 & 1812 & 1819 & 1822 & 1819 & 1822 & 1822 & 0.47 & 15.9 \\
\hline 2,6-Dinitrotoluene & 1842 & 1848 & 1854 & 1862 & 1866 & 1864 & 1870 & 1866 & 0.5 & 15.9 \\
\hline 1,2-Dinitrobenzene & 1890 & 1896 & 1903 & 1911 & 1914 & 1914 & 1920 & 1914 & 0.5 & 16.4 \\
\hline Acenapthylene & 1798 & 1809 & 1819 & 1827 & 1829 & 1829 & 1836 & 1832 & 0.66 & 16.5 \\
\hline 3-Nitroaniline & 1908 & 1915 & 1922 & 1930 & 1935 & 1934 & 1941 & 1936 & 0.56 & 16.5 \\
\hline Acenaphthene & 1830 & 1839 & 1849 & 1858 & 1861 & 1860 & 1863 & 1863 & 0.63 & 16.7 \\
\hline 2,4-dinitro-phenol & 1895 & 1902 & 1912 & 1920 & 1923 & 1922 & 1926 & 1923 & 0.54 & 15.9 \\
\hline Dibenzofuran & 1858 & 1868 & 1877 & 1887 & 1889 & 1889 & 1893 & 1892 & 0.63 & 16.4 \\
\hline 2,4-Dinitrotoluene & 1918 & 1925 & 1934 & 1944 & 1946 & 1943 & 1949 & 1946 & 0.54 & 16.2 \\
\hline 2,3,5,6-Tetrachlorophenol & 1864 & 1874 & 1883 & 1893 & 1892 & 1893 & 1894 & 1897 & 0.58 & 16.2 \\
\hline 2,3,4,6-Tetrachlorophenol & 1879 & 1889 & 1896 & 1908 & 1907 & 1907 & 1910 & 1916 & 0.61 & 16.2 \\
\hline Diethyl Phthalate & 1930 & 1938 & 1944 & 1955 & 1952 & 1953 & 1953 & 1955 & 0.46 & 15.9 \\
\hline Fluorene & 1947 & 1959 & 1970 & 1978 & 1981 & 1982 & 1985 & 1985 & 0.66 & 16.4 \\
\hline $\begin{array}{l}\text { 4-Chlorophenyl phenyl } \\
\text { ether }\end{array}$ & 1921 & 1929 & 1938 & 1948 & 1949 & 1949 & 1951 & 1952 & 0.55 & 16.2 \\
\hline 4-Nitroaniline & 2093 & 2101 & 2109 & 2117 & 2124 & 2124 & 2129 & 2122 & 0.56 & 16.2 \\
\hline $\begin{array}{l}\text { 4,6-Dinitro-2- } \\
\text { methylphenol }\end{array}$ & 1997 & 2003 & 2011 & 2022 & 2023 & 2024 & 2029 & 2026 & 0.55 & 16.5 \\
\hline Diphenylamine & 2007 & 2016 & 2025 & 2034 & 2039 & 2036 & 2042 & 2038 & 0.58 & 16.2 \\
\hline Azobenzene & 1975 & 1986 & 1994 & 2004 & 2007 & 2005 & 2007 & 2007 & 0.58 & 16.5 \\
\hline $\begin{array}{l}\text { 4-Bromophenyl phenyl } \\
\text { ether }\end{array}$ & 2061 & 2069 & 2079 & 2091 & 2091 & 2091 & 2094 & 2093 & 0.56 & 16.9 \\
\hline Hexachlorobenzene & 2046 & 2058 & 2066 & 2080 & 2078 & 2080 & 2082 & 2084 & 0.62 & 16.7 \\
\hline
\end{tabular}




\begin{tabular}{lrlllllllrr} 
Pentachloro-phenol & 2137 & 2148 & 2159 & 2170 & 2173 & 2172 & 2179 & 2176 & 0.65 & 16.9 \\
Anthracene & 2233 & 2246 & 2258 & 2268 & 2276 & 2274 & 2284 & 2276 & 0.72 & 17 \\
Phenanthrene & 2242 & 2256 & 2267 & 2277 & 2283 & 2283 & 2292 & 2284 & 0.7 & 17.2 \\
Carbazole & 2350 & 2364 & 2373 & 2383 & 2392 & 2390 & 2399 & 2390 & 0.65 & 16.7 \\
di-n-Butyl phthalate & 2304 & 2312 & 2317 & 2333 & 2326 & 2329 & 2325 & 2331 & 0.4 & 16 \\
Fluoranthene & 2606 & 2621 & 2637 & 2647 & 2658 & 2658 & 2668 & 2656 & 0.74 & 17.5 \\
Pyrene & 2703 & 2718 & 2736 & 2744 & 2757 & 2755 & 2769 & 2755 & 0.75 & 17.7 \\
Benzyl butyl phthalate & 2865 & 2877 & 2890 & 2905 & 2911 & 2906 & 2911 & 2907 & 0.56 & 16.5 \\
Bis(2-ethylhexyl) adipate & 2562 & 2563 & 2563 & 2592 & 2574 & 2573 & 2560 & 2581 & 0.41 & 15.7 \\
Benz[4.9anthracene & 3138 & 3155 & 3172 & 3185 & & & & & 0.57 & 9 \\
Chrysene & 3137 & 3185 & 3205 & 3220 & & & & & 0.98 & 9.7 \\
Bis(2-ethylhexyl) phthalate & 2859 & 2863 & 2866 & 2890 & & & & & 0.42 & 8.4 \\
\hline
\end{tabular}


Table 4.9. The second dimension linear retention indices ${ }^{2} I$ of compounds identified from MegaMix B

\begin{tabular}{|c|c|c|c|c|c|c|c|c|c|c|}
\hline Chromatographic conditions & & & & $\begin{array}{l}{ }^{2} I \\
\text { iu) }\end{array}$ & & & & & $\begin{array}{c}R S D \\
(\%)^{\mathrm{b}}\end{array}$ & $\begin{array}{c}\Delta^{2} T_{e} \\
\left({ }^{\circ} \mathrm{C}\right)^{\mathrm{c}}\end{array}$ \\
\hline Secondary oven offset $\left({ }^{\circ} \mathrm{C}\right)$ & +5 & +5 & +5 & +5 & +5 & +5 & +5 & +10 & & \\
\hline Ramp rate $\left({ }^{\circ} \mathrm{C} /\right.$ minute $)$ & 4 & 4.5 & 5 & 5 & 5.5 & 5.5 & 6 & 5 & & \\
\hline Modulation period (s) & 5 & 5 & 5 & 4 & 5 & 4 & 5 & 4 & & \\
\hline cis-1,3-Dichloro-1-Propene, $(\mathrm{Z})$ & 861 & 862 & 873 & 868 & & & & 865 & 0.5 & 11.5 \\
\hline trans-1,3-Dichloro-Propene $€$ & 900 & 899 & 903 & 907 & 900 & 900 & 903 & 901 & 0.27 & 10 \\
\hline Toluene & 872 & 873 & 884 & 878 & & & & 877 & 0.48 & 10 \\
\hline 1,1,2-Trichloroethane & 911 & 911 & 912 & 917 & 914 & 913 & 915 & 910 & 0.24 & 10.2 \\
\hline Ethyl methacrylate & 877 & 878 & 888 & 885 & & & & 882 & 0.46 & 10 \\
\hline 1,3-Dichloropropane & 930 & 930 & 933 & 934 & 931 & 931 & 932 & 929 & 0.19 & 10.5 \\
\hline Dibromochloro-Methane & 962 & 961 & 965 & 968 & 964 & 964 & 964 & 963 & 0.21 & 10.4 \\
\hline Tetrachloroethene & 898 & 898 & 903 & 905 & & & & 903 & 0.33 & 10.3 \\
\hline 1,2-Dibromoethane & 976 & 977 & 979 & 982 & 977 & 976 & 979 & 975 & 0.22 & 11.2 \\
\hline Chlorobenzene & 989 & 989 & 991 & 996 & 992 & 989 & 992 & 989 & 0.21 & 11.9 \\
\hline $1,1,1,2$-Tetrachloroethane & 973 & 981 & 984 & 991 & 986 & 984 & 983 & 984 & 0.48 & 12 \\
\hline Ethylbenzene & 978 & 978 & 982 & 986 & 978 & 977 & 979 & 979 & 0.29 & 12 \\
\hline p-Xylene & 986 & 984 & 986 & 993 & 983 & 983 & 989 & 986 & 0.33 & 12 \\
\hline Styrene & 1044 & 1043 & 1050 & 1052 & 1045 & 1045 & 1042 & 1048 & 0.31 & 11.5 \\
\hline 0 -Xylene & 1026 & 1025 & 1031 & & 1027 & 1027 & 1027 & 1024 & 0.22 & 12 \\
\hline Bromoform & 1102 & 1107 & 1111 & 1111 & 1112 & 1106 & 1113 & 1109 & 0.3 & 12.3 \\
\hline cis-1,4-Dichloro-2-butene, , (z)- & 1088 & 1089 & 1093 & 1096 & 1093 & 1091 & 1091 & 1091 & 0.21 & 12.4 \\
\hline 1,1,2,2-Tetrachloroethane & 1095 & 1094 & 1098 & 1099 & 1095 & 1094 & 1096 & 1090 & 0.24 & 12.7 \\
\hline 1,2,3-Trichloropropane & 1111 & 1112 & 1115 & 1121 & 1112 & 1110 & 1130 & 1115 & 0.56 & 13 \\
\hline Isopropylbenzene(cumene) & 1043 & 1039 & 1047 & 1050 & 1043 & 1044 & 1047 & 1045 & 0.28 & 13 \\
\hline trans-1,4-Dichloro-2-butene, $(\mathrm{E})$ - & & & 1129 & 1134 & 1127 & 1127 & 1116 & 1128 & 0.48 & 6.4 \\
\hline Bromobenzene & 1113 & 1115 & 1117 & 1121 & 1117 & 1117 & 1118 & 1115 & 0.19 & 13.2 \\
\hline 2-Chlorotoluene & 1110 & 1112 & 1115 & 1120 & 1114 & 1114 & & 1117 & 0.28 & 12 \\
\hline n- Propylbenzene & 1076 & 1079 & 1083 & 1087 & 1079 & 1079 & 1083 & 1084 & 0.31 & 13 \\
\hline
\end{tabular}




\begin{tabular}{|c|c|c|c|c|c|c|c|c|c|c|}
\hline 4-Chlorotoluene & 1115 & 1114 & 1119 & 1121 & 1116 & 1115 & 1118 & 1118 & 0.19 & 13.4 \\
\hline 1,3,5-Trimethylbenzene & 1092 & 1091 & 1098 & 1100 & 1094 & 1095 & 1091 & 1092 & 0.28 & 13.9 \\
\hline Pentachloroethane & 1144 & 1144 & 1149 & 1150 & 1149 & 1148 & 1148 & 1142 & 0.23 & 13.7 \\
\hline tert-Butylbenzene & 1112 & 1115 & 1121 & 1125 & 1117 & 1117 & 1118 & 1121 & 0.33 & 13.9 \\
\hline 1,2,4-Trimethylbenzene & 1132 & 1132 & 1137 & 1141 & 1136 & 1134 & & 1137 & 0.26 & 12 \\
\hline sec-Butylbenzene & 1135 & 1133 & 1137 & 1143 & 1138 & 1137 & 1135 & 1137 & 0.24 & 14.2 \\
\hline p-Cymene(4-isopropyl toluene & 1147 & 1148 & 1152 & 1158 & 1149 & 1149 & 1152 & 1152 & 0.28 & 15 \\
\hline n-Butylbenzene & 1185 & 1184 & 1189 & 1196 & 1188 & 1188 & 1189 & 1192 & 0.29 & 14 \\
\hline 1,2-Dibromo-3-Chloropropane & 1329 & 1334 & 1340 & 1345 & 1343 & 1341 & 1344 & 1345 & 0.41 & 14.5 \\
\hline Benzene, 1,2,3-trichloro- & 1452 & 1459 & 1464 & 1471 & 1470 & 1469 & 1474 & 1472 & 0.48 & 15.2 \\
\hline 1,3-Dichlorobenzene* & 1181 & 1184 & 1188 & 1196 & 1189 & 1188 & 1187 & 1191 & 0.34 & 14.2 \\
\hline 1,4-Dichlorobenzene* & 1197 & 1200 & 1201 & 1211 & 1205 & 1204 & 1206 & 1207 & 0.34 & 14.5 \\
\hline 1,2-Dichlorobenzene* & 1233 & 1237 & 1241 & 1247 & 1243 & 1242 & 1242 & 1246 & 0.34 & 15.9 \\
\hline Nitrobenzene* & 1347 & 1353 & 1359 & 1364 & 1363 & 1360 & 1365 & 1364 & 0.44 & 14.2 \\
\hline Benzene, 1,2,4-trichloro-* & 1394 & 1398 & 1403 & 1411 & 1406 & 1406 & 1408 & 1412 & 0.4 & 15.2 \\
\hline Naphthalene* & 1446 & 1452 & 1460 & 1465 & 1466 & 1463 & 1467 & 1467 & 0.51 & 15.9 \\
\hline $\begin{array}{l}\text { 1,3-Butadiene, 1,1,2,3,4,4- } \\
\text { hexachloro-* }\end{array}$ & 1374 & 1376 & 1381 & 1389 & 1383 & 1382 & 1384 & 1387 & 0.35 & 15.7 \\
\hline
\end{tabular}


The column used in GC-MS was a DB-17ms column, which was the same as the second dimension column used in GC×GC-MS.

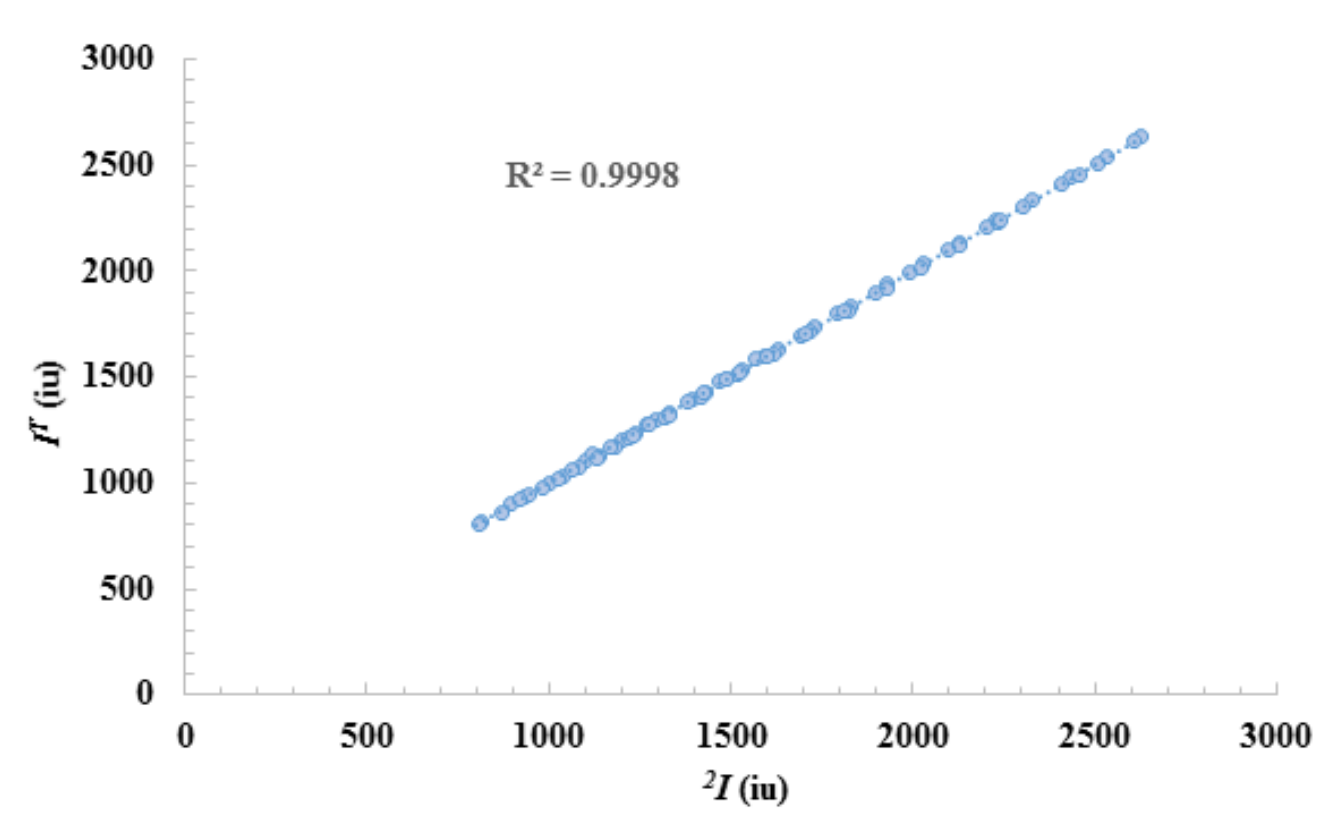

Figure 4.7. Correlation between the linear retention index, $I^{T}$, of compounds calculated from GC-MS data and their corresponding second dimension linear retention indices ${ }^{2} I$ calculated using the method developed in this study. The compounds include 80 compound standards and compounds in MegaMix A and MegaMix B

Figure 4.7 shows the overall relation of the retention indices calculated by the surface fitting method and the linear retention indices obtained from the GC-MS data $\left(R^{2}=0.9998\right)$. The retention index difference between the ${ }^{2} I$ values of the 80 compound standards ranged from $0.0 \mathrm{iu}$ to $19.0 \mathrm{iu}$, with an average linear retention index difference of $5.1 \mathrm{iu}$. The 
MegaMix A and MegaMix B had a wide range of retention index difference with an average variation of 23.9 iu because they are composed of a wide variety of chemical structures from different chemical classes, including branching and cyclization.

The retention index difference between the linear retention index $I^{T}$ calculated from GC-MS data and the ${ }^{2} I$ calculated from GC $\times$ GC-MS data can be attributed to the polarity of the molecule, instrumental variation, chemical structure of the molecule, length of the column, effect of the transfer line and column temperature. ${ }^{78-81}$ Previous study demonstrated that the retention index of an analyte generally increases with the increase of elution temperature. ${ }^{79} \mathrm{We}$ observed that some of the identified compounds followed this trend, while other compounds did not. The reason is that temperature dependency of retention index is also related to the structure of an analyte. ${ }^{78-81}$ Table 4.10 shows an example of elution temperature of the 80 compound standards, where the elution temperature of a compound was increased with the increase of temperature ramp rate. The lowest elution temperature corresponded to the ramp rate of $4{ }^{\circ} \mathrm{C} / \mathrm{min}$ and the highest elution temperature corresponded to the ramp rate of $6{ }^{\circ} \mathrm{C} / \mathrm{min}$. The temperature range was calculated by subtracting the elution temperature of the ramp rate $4{ }^{\circ} \mathrm{C} / \mathrm{min}$ from the elution temperature of the ramp rate $6{ }^{\circ} \mathrm{C} / \mathrm{min}$. The average range of the elution temperature for the 80 compound standards was $14.5^{\circ} \mathrm{C}$. A similar effect was also observed for compounds in MegaMix A and MegaMix B, where most of the compounds showed a trend of increasing calculated retention index with the increasing of elution temperature, with an average range of elution temperature $18.5^{\circ} \mathrm{C}$. 
Table 4.10. The second dimension elution temperature ${ }^{2} T_{e}$ of the 80 standard compounds with different ramp rates

\begin{tabular}{|c|c|c|c|c|c|c|}
\hline \multirow{2}{*}{$\begin{array}{l}\text { Compounds name } \\
\text { Ramp }\left({ }^{\circ} \mathrm{C} / \mathrm{min}\right)\end{array}$} & \multicolumn{5}{|c|}{${ }^{2} I(\mathrm{iu})^{\mathrm{a}}$} & \multirow[t]{2}{*}{$\Delta^{2} \mathrm{~T}_{\mathrm{e}}^{b}$} \\
\hline & 4 & 4.5 & 5 & 5.5 & 6 & \\
\hline \multicolumn{7}{|l|}{ FAME } \\
\hline Methyl butyrate & 84.8 & 86.8 & 89.3 & 91.2 & 93.6 & 8.9 \\
\hline Methyl valerate & 92.1 & 94.7 & 97.6 & 100.0 & 102.6 & 10.5 \\
\hline Methyl caproate & 102.4 & 106.0 & 108.9 & 111.9 & 115.1 & 12.7 \\
\hline Methyl enanthate & 115.8 & 119.5 & 123.0 & 126.1 & 129.6 & 13.9 \\
\hline Methyl caprylate & 129.8 & 133.8 & 137.6 & 141.2 & 144.7 & 14.9 \\
\hline Methyl pelargonate & 144.5 & 148.4 & 152.2 & 155.4 & 159.2 & 14.7 \\
\hline Methyl caprate & 158.1 & 162.3 & 166.0 & 169.6 & 173.2 & 15.0 \\
\hline Methyl undecanoate & 171.5 & 175.4 & 179.3 & 182.9 & 186.7 & 15.2 \\
\hline Methyl laurate & 183.8 & 188.1 & 191.8 & 195.8 & 199.2 & 15.4 \\
\hline Methyl tridecanoate & 195.8 & 199.8 & 203.9 & 207.7 & 211.2 & 15.4 \\
\hline Methyl myristate & 207.1 & 211.4 & 215.1 & 218.7 & 222.7 & 15.5 \\
\hline $\begin{array}{l}\text { Methyl } \\
\text { pentadecanoate }\end{array}$ & 217.8 & 221.9 & 226.0 & 229.7 & 233.2 & 15.4 \\
\hline Methyl palmitate & 227.8 & 232.0 & 236.4 & 239.8 & 243.7 & 15.9 \\
\hline Methyl margarate & 237.8 & 242.1 & 246.0 & 249.9 & 253.7 & 15.9 \\
\hline Methyl stearate & 247.1 & 251.1 & 255.1 & 259.0 & 262.7 & 15.5 \\
\hline Methyl nonadecanoate & 255.8 & 260.1 & 264.3 & 268.2 & 271.7 & 15.9 \\
\hline Methyl arachidate & 264.5 & 268.8 & 272.6 & 276.4 & 280.2 & 15.7 \\
\hline $\begin{array}{l}\text { Methyl } \\
\text { heneicosanoate }\end{array}$ & 272.8 & 277.0 & 281.0 & 284.7 & 288.7 & 15.9 \\
\hline Methyl behenate & 280.5 & 284.9 & 288.9 & & & \\
\hline Methyl tricosanoate & 288.1 & & & & & \\
\hline \multicolumn{7}{|l|}{ Fatty acid } \\
\hline Butyric acid & 88.1 & 91.0 & 93.4 & 95.8 & 98.1 & 10.0 \\
\hline Valeric acid & 97.4 & 100.7 & 103.9 & 106.8 & 109.6 & 12.2 \\
\hline Caproic acid & 109.4 & 113.1 & 116.8 & 120.1 & 123.1 & 13.7 \\
\hline Enanthic acid & 123.1 & 127.0 & 131.0 & 134.4 & 137.7 & 14.5 \\
\hline Caprylic acid & 137.8 & 142.0 & 145.6 & 149.0 & 152.7 & 14.9 \\
\hline Pelargonic acid & 150.5 & 154.8 & 158.9 & 161.9 & 165.2 & 14.7 \\
\hline Capric acid & 164.1 & 167.9 & 172.2 & 175.2 & 178.7 & 14.5 \\
\hline Undecylic acid & 176.5 & 180.6 & 184.7 & 188.0 & 191.7 & 15.2 \\
\hline Lauric acid & 188.8 & 192.6 & 196.8 & 200.4 & 203.7 & 14.9 \\
\hline Tridecylic acid & 200.1 & 204.3 & 208.5 & 211.8 & 215.2 & 15.0 \\
\hline Myristic acid & 210.8 & 215.1 & 219.3 & 222.8 & 226.7 & 15.9 \\
\hline Pentadecylic acid & 221.5 & 225.6 & 230.1 & 233.4 & 237.2 & 15.7 \\
\hline Palmitic acid & 231.5 & 235.8 & 240.1 & 243.5 & 247.2 & 15.7 \\
\hline Margaric acid & 241.1 & 245.1 & 249.7 & 253.1 & 256.7 & 15.5 \\
\hline
\end{tabular}




\begin{tabular}{|c|c|c|c|c|c|c|}
\hline Stearic acid & 250.1 & 254.5 & 258.9 & 262.2 & 266.2 & 16.0 \\
\hline Nonadecylic acid & 259.1 & 263.1 & 267.7 & 271.4 & 274.7 & 15.5 \\
\hline Arachidic acid & 267.8 & 271.8 & 276.4 & 279.7 & 283.7 & 15.9 \\
\hline Heneicosylic acid & 275.8 & 280.0 & 284.3 & 287.9 & & \\
\hline Behenic acid & 283.8 & 287.9 & & & & \\
\hline \multicolumn{7}{|l|}{ Ketone } \\
\hline 2-Pentanone & 83.1 & 85.3 & 87.2 & 89.4 & 91.6 & 8.5 \\
\hline 2-Hexanone & 89.1 & 91.7 & 94.3 & 96.8 & 99.1 & 10.0 \\
\hline 2-Heptanone & 98.4 & 101.9 & 104.7 & 107.8 & 110.6 & 12.2 \\
\hline 2-Octanone & 111.1 & 114.6 & 118.1 & 121.5 & 124.7 & 13.5 \\
\hline 2-Nonanone & 125.5 & 129.3 & 133.1 & 136.2 & 139.7 & 14.2 \\
\hline 2-Decanone & 139.8 & 143.9 & 147.6 & 151.3 & 154.7 & 14.9 \\
\hline 2-Undecanone & 154.1 & 158.1 & 162.2 & 165.5 & 169.2 & 15.0 \\
\hline 2-Dodecanone & 167.8 & 172.0 & 175.6 & 179.3 & 183.2 & 15.4 \\
\hline 2-Tridecanone & 180.5 & 184.8 & 188.5 & 192.1 & 196.2 & 15.7 \\
\hline 2-Tetradecanone & 192.8 & 196.8 & 201.0 & 204.5 & 208.2 & 15.4 \\
\hline 2-Pentadecanone & 204.1 & 208.4 & 212.6 & 216.4 & 220.2 & 16.0 \\
\hline 2-Hexadecanone & 215.1 & 219.6 & 223.5 & 227.4 & 231.2 & 16.0 \\
\hline 2-Heptadecanone & 225.8 & 230.1 & 234.3 & 237.9 & 241.7 & 15.9 \\
\hline 2-Octadecanone & 235.8 & 239.9 & 243.9 & 247.6 & 251.7 & 15.9 \\
\hline 2-Nonadecanoe & 245.1 & 249.6 & 253.5 & 257.2 & 261.2 & 16.0 \\
\hline \multicolumn{7}{|l|}{ Alcohol } \\
\hline 1-Pentanol & 87.4 & 89.9 & 92.6 & 94.9 & 97.1 & 9.7 \\
\hline 1-Hexanol & 96.4 & 99.2 & 102.2 & 105.0 & 108.1 & 11.7 \\
\hline 1-Heptanol & 108.8 & 112.0 & 115.5 & 119.2 & 122.1 & 13.4 \\
\hline 1-Octanol & 122.5 & 125.9 & 129.7 & 133.4 & 136.6 & 14.2 \\
\hline 1-Nonanol & 137.1 & 141.6 & 148.9 & 151.8 & 157.1 & 20.0 \\
\hline 1-Undecanol & 168.4 & 171.6 & 178.0 & 180.2 & 186.1 & 17.7 \\
\hline 1-Dodecanol & 186.1 & 185.1 & 192.6 & 196.2 & 200.6 & 14.5 \\
\hline \multicolumn{7}{|l|}{ Iodobutne } \\
\hline 1-Iodobutane & 92.1 & 95.1 & 98.0 & 100.4 & 103.1 & 11.0 \\
\hline 1-Iodopentane & 103.1 & 106.7 & 109.7 & 112.8 & 116.2 & 13.0 \\
\hline 1-Iodohexane & 117.1 & 120.6 & 124.3 & 127.9 & 131.2 & 14.0 \\
\hline 1-Iodoheptane & 131.8 & 136.0 & 139.7 & 143.5 & 146.7 & 14.9 \\
\hline 1-Iodooctane & 146.8 & 151.0 & 155.1 & 158.7 & 162.2 & 15.4 \\
\hline 1-Iodononane & 161.5 & 165.6 & 169.7 & 173.3 & 177.2 & 15.7 \\
\hline 1-Iododecane & 175.1 & 179.5 & 183.5 & 187.1 & 191.2 & 16.0 \\
\hline 1-Iodoundecane & 188.1 & 192.3 & 196.4 & 200.4 & 204.2 & 16.0 \\
\hline 1-Iodododecane & 200.5 & 204.6 & 208.9 & 212.7 & 216.2 & 15.7 \\
\hline 1-Iodohexadecane & 243.5 & 247.8 & 251.8 & 255.8 & 259.7 & 16.2 \\
\hline 1-Iodooctadecane & 261.8 & 266.5 & 270.6 & 274.6 & 278.2 & 16.4 \\
\hline \multicolumn{7}{|l|}{ Aldehyde } \\
\hline Pentanal & 83.8 & 85.7 & 88.0 & 90.3 & 92.1 & 8.4 \\
\hline Hexanal & 90.1 & 92.9 & 95.5 & 98.1 & 100.6 & 10.5 \\
\hline Heptanal & 100.1 & 103.4 & 106.8 & 109.6 & 112.6 & 12.5 \\
\hline
\end{tabular}




\begin{tabular}{|l|r|r|r|r|r|r|}
\hline Octanal & 113.1 & 116.9 & 120.6 & 123.8 & 127.2 & 14.0 \\
\hline Nonanal & 127.5 & 131.5 & 135.1 & 138.5 & 142.2 & 14.7 \\
\hline Decanal & 142.1 & 146.1 & 150.1 & 153.6 & 157.2 & 15.0 \\
\hline Undecanal & 156.1 & 160.4 & 164.3 & 167.8 & 171.7 & 15.5 \\
\hline Dodecanal & 169.8 & 173.9 & 178.1 & 181.6 & 185.2 & 15.4 \\
\hline
\end{tabular}

\subsubsection{Reproducibility and Robustness of Surface Fitting}

To study the reproducibility and robustness of the developed method, we analyzed the 80 compound standards, MegaMix A and MegaMix B in different instrumental conditions by varying the temperature ramp rate of $\mathrm{GC} \times \mathrm{GC}-\mathrm{MS}$. We first analyzed the 80 compound standards in five ramp rates. The standard deviation $(S T D)$ of the ${ }^{2} I$ calculated from the five analyses ranged from 0.0 to 9.0 iu. Figure 4.8 shows the distribution of the STD of the 80 compound standards.

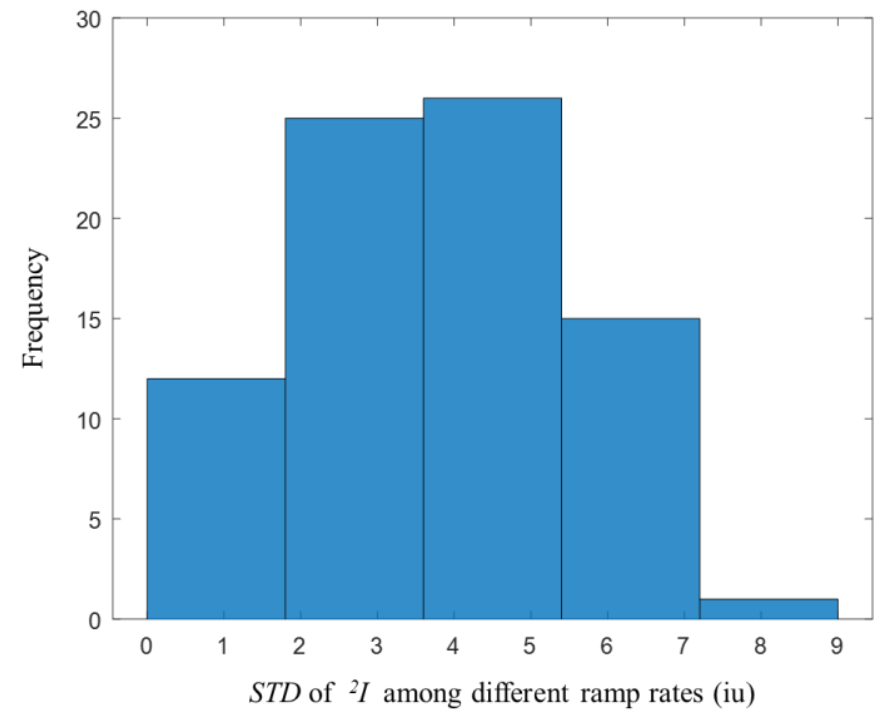

Figure 4.8. Distribution of standard deviation of the calculated ${ }^{2} I$ of the 80 standard compounds among different ramp rates. 
Among the 80 compound standards, the STD of 64 compounds were within $5 \mathrm{iu}$; 15 compounds within 6-7 iu; and 1 compound within 8-9 iu. Overall, the mean standard deviation $(m S T D)$ and relative standard deviation $(R S D)$ of ${ }^{2} I$ was 3.8 iu and $0.25 \%$, respectively. An average relative standard deviation $(R S D)$ of the ${ }^{2} I$ for compounds in MegaMix A and MegaMix B was $0.51 \%$ and $0.32 \%$, respectively. The small $R S D$ of ${ }^{2} I$ demonstrates that the developed method is robust in calculating the second dimension linear retention index of compounds analyzed in different instrumental conditions.

This study is a continuation of our previous work, i.e., calculating the second dimension retention index in GC $\times$ GC-MS via surface fitting. ${ }^{105}$ The method developed in this study considered the second dimension separation in temperature-programmed mode and directly calculated the linear retention index via surface fitting. Furthermore, the current method used $\mathrm{C}_{4}-\mathrm{C}_{25}$ FAMEs as the reference series while keeping the retention index in $n$-alkane scale. This approach allowed using any compound as the reference series where needed. Therefore, the current method is not limited to any reference series and allowed much more scope to maneuver the reference series selection based on the need.

\subsection{Conclusions}

We developed a surface fitting method for calculating the linear retention index ${ }^{2} I$ of compounds separated by GC $\times$ GC-MS from their second dimension retention time ${ }^{2} t_{M}$ and the secondary column temperature ${ }^{2} T_{e}$, using $\mathrm{C}_{4}-\mathrm{C}_{25}$ FAMEs as the reference series and keeping the retention index in $n$-alkane scale. The developed method showed an excellent agreement with the $n$-alkane based method. The standard deviation of the ${ }^{2} I$ of $n$-alkanes ranged from 3.6 to $14.0 \mathrm{iu}$ with an average variation of $11.8 \mathrm{iu}$. The average relative 
standard deviation of the ${ }^{2} I$ for compounds in MegaMix A and MegaMix B was $0.47 \%$ and $0.33 \%$, respectively. Although we used only $\mathrm{C}_{4}-\mathrm{C}_{25}$ FAMEs as the reference series to create a spacious second dimension retention map, it is possible to use any compounds as references to construct the retention index surface and calculate the ${ }^{2} I$ of analytes.

A future endeavor would be the use of mixture of reference compounds having different degree of polarity and diverse structure along with some individual reference compounds in order to construct the second dimension retention index surface. In addition, besides the MATLAB based surface fitting approach, other statistical methods i.e. machine learning algorithm can be employed to establish the relationship between the second dimension elution temperate, second dimension retention time and the corresponding second dimension retention index. From this relationship, second dimension retention index of unknown analytes can be calculated. 


\title{
CHAPTER 5
}

\author{
INTEGRATING COMPREHENSIVE TWO-DIMENSIONAL GAS \\ CHROMATOGRAPHY MASS SPECTROMETRY AND PARALLEL TWO- \\ DIMENSIONAL LIQUID CHROMATOGRAPHY MASS SPECTROMETRY FOR \\ UNTRARGETED METABOLOMICS
}

\subsection{Introduction}

Metabolites are the intermediates and products of all biological processes that take place in a biological system. Metabolites can be polar or non-polar, as well as organic or inorganic compounds. ${ }^{3,5,107,108}$ The diverse chemical characteristics and huge number of entities make metabolite separation challenging in metabolomics. Separation methods such as liquid chromatography (LC) and gas chromatography (GC) are usually coupled with mass spectrometry (MS) to increase the metabolite coverage. ${ }^{109}$ Due to the limited peak capacity of a single column, one dimensional separation method using LC or GC can only resolve a limited number of metabolites in a biological sample. ${ }^{110}$ In order to obtain better separation and higher peak capacity, multi-dimensional separation methods have been developed and applied in metabolomics even though the multi-dimensional separation usually needs long separation time and reduces the sample throughput. ${ }^{111-114}$ 
Comprehensive two-dimensional gas chromatography mass spectrometry (GC×GCMS) uses two capillary GC columns with different stationary phases for separation of metabolites. ${ }^{60-62,115,116}$ The two columns are usually connected via a thermal modulator. The second column is typically much shorter than the first (i.e., 1-2 $\mathrm{m}$ as opposed to $30-60$ $\mathrm{m}$ for the first column) and is generally operated at a higher temperature. In the case of metabolites that co-elute from the first column, the second column may allow for further separation due to the different stationary phases as well as the different column temperatures. Thus, GC $\times \mathrm{GC}-\mathrm{MS}$ provides superior chromatographic peak capacity, selectivity, and lower detection limit for analysis of metabolites.

Two-dimensional liquid chromatography (2DLC) is usually configured in either a heart-cutting mode or a comprehensive mode..$^{50,117,118}$ The heart-cutting analysis involves the collection of a few peaks of interest from the elution of the first dimension column, and then subjecting only those peaks onto the second dimension column for further separation. This configuration can increase signal-to-noise ratio and improve sensitivity for targeted metabolites. ${ }^{51}$ In the comprehensive configuration, eluate from the first dimension column is collected into multiple fractions and each fraction is subjected to the second dimension column for further separation. The comprehensive 2DLC offers an increased resolution. ${ }^{52}$ Most of the 2DLC systems are operated in online mode, where the second dimension separation is carried out simultaneously with the first dimension separation. ${ }^{53}$ While the online mode of 2DLC has many advantages such as improved reliability and sample throughput, shortened analysis time and minimum sample loss, it requires the second dimension analysis to be completed during the time needed to collect and transfer a fraction from the first dimension column except that the 2DLC was configured in a stop-flow 
mode. ${ }^{119}$ Another limitation of the online 2DLC technique is that the mobile phases used in the two columns must be compatible in both miscibility and solvent strength. In addition, some metabolites may partition between the fractions collected from the first dimension column, resulting in large variation in metabolite quantification and even metabolite identification.

Klavins, et al. developed a parallel 2DLC system to perform orthogonal hydrophilic interaction chromatography (HILIC) and reversed-phase chromatography (RPC) in one analytical run, where a sample was first delivered to two sample loops during sample loading. ${ }^{54}$ The two sample aliquots were then simultaneously injected onto a dual column setup, and parallel separations were performed on the two columns. The eluates from the two columns were then merged and subjected to a mass spectrometer for further analysis. This strategy is simple yet effective for coupling HILIC and RPC for the purpose of decreasing analysis time and increasing throughput. Furthermore, the parallel 2DLC-MS configuration allows the use of two long columns and gradient time to increase separation power. However, parallel 2DLC-MS has the potentials of peak overlapping which incurs resolution problem. On the other hand, unlike the comprehensive 2DLC-MD, parallel 2DLC-MS does not suffer from the peak partition and solvent miscibility and solvent strength issues.

While the effectiveness of both GC×GC-MS and 2DLC-MS for metabolomics has been separately demonstrated in multiple studies ${ }^{82,115,120-122}$, analysis of biological samples on the two platforms has not yet been explored. In the current study, we aimed to integrate the GC $\times$ GC-MS and the parallel 2DLC-MS for wider metabolite coverage, high confidence of metabolite identification and quantification, as well as high confidence of metabolic 
pathway assignment. The performance of developed system was tested by analyzing polar metabolites extracted from rat livers, where each metabolite extract was analyzed by GC $\times$ GC-MS and parallel 2DLC-MS, respectively. After metabolite identification and quantification, the performance of GC×GC-MS and parallel 2DLC-MS was assessed based on the number of identified metabolites, the accuracy of metabolite quantification, and the extent of their pathway coverage.

\subsection{Experimental}

\subsubsection{Animal Treatment}

Thirteen male weanling Sprague-Dawley rats (35-45 g) from the Harlan Laboratories (Indianapolis, IN) were fed (ad lib) a purified AIN-76 diet with a defined copper content in form of cupric carbonate. The animals were housed in stainless steel cages in a temperature and humidity controlled room with a 12:12 h light-dark cycle. The 13 rats formed two groups, Group $1(\mathrm{G} 1, \mathrm{n}=6)$ and Group $2(\mathrm{G} 2, \mathrm{n}=7)$. The rats in G1 received adequate dose of copper $(6.0 \mathrm{ppm})$ with free access to deionized water. The rats in G2 received supplemental dose of copper $(20 \mathrm{ppm})$ with free access to deionized water containing 30\% fructose (w/v). Fructose enriched drinking water was changed twice each week. All animals were fed for 4 weeks. At the end of the feeding period, each rat was killed under anesthesia with pentobarbital ( $50 \mathrm{mg} / \mathrm{kg}$ I.P. injection) after overnight fasting. Portions of rat liver were fixed with $10 \%$ formalin for subsequent sectioning, while others were snap-frozen with liquid nitrogen. All studies were approved by the University of Louisville Institutional Animal Care and Use Committee, which is certified by the American Association of Accreditation of Laboratory Animal Care.

\subsubsection{Metabolite Sample Preparation}


All samples were processed in random order to avoid systemic bias. After placing about $100 \mathrm{mg}$ of liver tissue in a 1.5-mL Eppendorf tube, water was added at a ratio of $100 \mathrm{mg}$ liver/mL water. After that, glass beads were added to the tube and the sample was homogenized using a Retsch MM 200 model mixer mill (Fisher Scientific, Hampton, NH, USA). To extract polar metabolites for GC $\times$ GC-MS analysis, $800 \mu \mathrm{L}$ methanol was added to $200 \mu \mathrm{L}$ homogenized liver. The mixture was vortex-mixed for $2 \mathrm{~min}$ and then placed on ice for $10 \mathrm{~min}$. After another 2 min of vortex mixing, the sample was centrifuged at 15,000 rpm for $20 \mathrm{~min}$ at $4{ }^{\circ} \mathrm{C}$. Seven hundred micro-liters of supernatant was transferred into a glass vial and dried in a SpeedVac evaporator to remove methanol, followed by lyophilization to remove water. The dried metabolite extract was then dissolved with 30 $\mu \mathrm{L}$ of $20 \mathrm{mg} / \mathrm{mL}$ methoxyamine hydrochloride pyridine solution followed by vigorous vortex mixing for $1 \mathrm{~min}$. Methoxymation was carried out by sonicating the sample for 20 min and incubating it at $60{ }^{\circ} \mathrm{C}$ for $1 \mathrm{~h}$. Derivatization was conducted by adding $30 \mu \mathrm{L}$ of N-tert-butyldimethylsilyl-N-methyltrifluoroacetamide $\quad$ with $\quad 1 \% \quad$ tertbutyldimethylchlorosilane to the glass vial. After $1 \mathrm{~h}$ incubation at $60{ }^{\circ} \mathrm{C}$, the mixture was transferred to a GC vial for analysis. A pooled sample was prepared by mixing $30 \mu \mathrm{L}$ of derivatized metabolite extract from each sample.

To extract polar metabolites for parallel 2DLC-MS analysis, $400 \mu \mathrm{L}$ methanol was added to $100 \mu \mathrm{L}$ homogenized liver. The mixtures was vortex-mixed and centrifuged using the same extraction protocol used for GC $\times$ GC-MS analysis. Three hundred micro-liters of supernatant was transferred into a glass vial. Methanol in the sample was removed using a SpeedVac evaporator and water was removed by lyophilization. The dried metabolite extract was reconstituted with $100 \mu \mathrm{L} 20 \%$ acetonitrile. The reconstitution was 
immediately preceded the parallel 2DLC-MS analysis. A pooled sample was prepared by mixing $50 \mu \mathrm{L}$ of metabolite extract from every sample of the same group.

\subsubsection{GC $\times$ GC-MS and Its Data Analysis}

A LECO (St. Joseph, MI, USA) Pegasus GC×GC-MS instrument was coupled with an Agilent 6890 gas chromatography and a Gerstel MPS2 auto-sampler (GERSTEL Inc., Linthicum, MD, USA), featuring a LECO two-stage cryogenic modulator and a secondary oven. The primary column was a $60 \mathrm{~m} \times 0.25 \mathrm{~mm}{ }^{1} \mathrm{~d}_{\mathrm{c}} \times 0.25 \mu \mathrm{m}{ }^{1} \mathrm{~d}_{\mathrm{f}}$ DB-5 ms capillary column (phenyl arylene polymer virtually equivalent to (5\%-phenyl)-methylpolysiloxane). The secondary GC column was a $1 \mathrm{~m} \times 0.25 \mathrm{~mm}{ }^{2} \mathrm{~d}_{\mathrm{c}} \times 0.25 \mu \mathrm{m}{ }^{2} \mathrm{~d}_{\mathrm{f}}$ DB-17 ms column ((50\% phenyl)-methylpolysiloxane) that was placed inside the secondary GC oven following the thermal modulator. Both columns were obtained from Agilent Technologies (Agilent Technologies J\&W, Santa Clara, CA, USA). The helium carrier gas (99.999\% purity) flow rate was set to $2.0 \mathrm{~mL} / \mathrm{min}$ at a corrected constant flow with pressure ramps. The inlet temperature was set to $280^{\circ} \mathrm{C}$. The primary column temperature was programmed with an initial temperature of $60{ }^{\circ} \mathrm{C}$ for $0.5 \mathrm{~min}$, then ramped at $5{ }^{\circ} \mathrm{C} / \mathrm{min}$ to $270{ }^{\circ} \mathrm{C}$, and maintained at $270{ }^{\circ} \mathrm{C}$ for $15 \mathrm{~min}$. The secondary column temperature program was set to an initial temperature of $70{ }^{\circ} \mathrm{C}$ for $0.5 \mathrm{~min}$ and then ramped at the same temperature gradient used in the first column to $280{ }^{\circ} \mathrm{C}$. The thermal modulator was $+15^{\circ} \mathrm{C}$ compared with the primary oven. The other instrument parameters were as: modulation period $2 \mathrm{~s}$, mass range $29-800 \mathrm{~m} / \mathrm{z}$, spectrum acquisition rate 200 mass spectra per second, ion source chamber temperature $230{ }^{\circ} \mathrm{C}$, transfer line temperature $280{ }^{\circ} \mathrm{C}$, detector voltage $1420 \mathrm{~V}$, electron energy $70 \mathrm{eV}$, and split ratio 20:1. The acceleration voltage was turned on after a solvent delay of $640 \mathrm{~s}$. 
The pooled sample was analyzed by GC×GC-MS eight times. The experiment data of the pooled sample were used to monitor the instrument variation. In addition, an aliquot of $\mathrm{C}_{7}-\mathrm{C}_{30} n$-alkane series was analyzed for retention index calculation.

To analyze the GC×GC-MS data, LECO's instrument control software, ChromaTOF (version 4.21), was used for peak picking and tentative metabolite identification. The threshold of spectral similarity score was set as $\geq 500$ with a maximum value of 1000 . MetPP software was used for retention index matching, peak merging, peak list alignment, normalization and statistical significance test. ${ }^{123,124}$ The $p$-value threshold was set as $p \leq$ 0.001 for retention index matching.

To verify the tentative identification of metabolites detected with significant abundance difference between groups, commercially available authentic standards of those compounds were analyzed by GC $\times$ GC-MS under the identical experimental conditions as those used for analyses of biological samples. A tentative metabolite assignment was considered as a correct identification only if the experimental information of the authentic metabolite agreed with the corresponding information of a chromatographic peak in the biological samples, i.e., difference of the first dimension retention time $\leq 10 \mathrm{~s}$, difference of the second dimension retention time $\leq 0.06 \mathrm{~s}$, and the mass spectral similarity $\geq 500$.

\subsubsection{Parallel 2DLC-MS and Its Data Analysis}

All samples were randomly analyzed on a Thermo Q Exactive HF Hybrid QuadrupoleOrbitrap Mass Spectrometer coupled with a Thermo UltiMate 3000 HPLC system (Thermo Fisher Scientific, Inc., Germany). The UltiMate 3000 HPLC system was equipped with a hydrophilic interaction chromatography (HILIC) column and a reversed-phase chromatography (RPC) column that were configured in parallel mode. ${ }^{54}$ The HILIC 
column (SeQuant ${ }^{\circledR}$ ZIC®-cHILIC, $150 \times 2.1 \mathrm{~mm}$ i.d., $\left.3 \mu \mathrm{m}\right)$ was purchased from EMD Millipore (Darmstadt, Germany). The RPC column (ACQUITY UPLC HSS T3, 150×2.1 mm i.d., $1.8 \mu \mathrm{m}$ ) was purchased from Waters Corp. (Milford, MA, USA). The temperature of those two columns was each set to $40{ }^{\circ} \mathrm{C}$. The HILIC column was operated as follows: mobile phase A was $10 \mathrm{mM}$ ammonium acetate ( $\mathrm{pH}$ adjusted to 3.25 with acetate) in water and mobile phase $\mathrm{B}$ was $100 \%$ acetonitrile with $0.1 \%$ formic acid. The gradient was: 0.0 $\min , 95 \% \mathrm{~B}$; 0.0 to $5.0 \mathrm{~min}, 95 \% \mathrm{~B}$ to $35 \% \mathrm{~B} ; 5.0$ to $6.0 \mathrm{~min}, 35 \% \mathrm{~B} ; 6.0$ to $6.1 \mathrm{~min}, 35 \%$ $\mathrm{B}$ to $5 \% \mathrm{~B} ; 6.1$ to $23.0 \mathrm{~min}, 5 \% \mathrm{~B} ; 23.0$ to $23.1 \mathrm{~min}, 5 \% \mathrm{~B}$ to $95 \% \mathrm{~B} ; 23.1$ to $40.0 \mathrm{~min}$, $95 \% \mathrm{~B}$. The flow rate was set to $0.3 \mathrm{~mL} / \mathrm{min}$. For the RPC column, the mobile phase A was water with $0.1 \%$ formic acid and mobile phase B was $100 \%$ acetonitrile with $0.1 \%$ formic acid. The gradient was as follows: $0.0 \mathrm{~min}, 5 \% \mathrm{~B} ; 0.0$ to $5.0 \mathrm{~min}, 5 \% \mathrm{~B} ; 5.0$ to $6.1 \mathrm{~min}$, $5 \% \mathrm{~B}$ to $15 \% \mathrm{~B} ; 6.1$ to $10.0 \mathrm{~min}, 15 \% \mathrm{~B}$ to $60 \% \mathrm{~B} ; 10.0$ to $12.0 \mathrm{~min}, 60 \% \mathrm{~B} ; 12.0$ to 14.0 $\min , 60 \% \mathrm{~B}$ to $100 \% \mathrm{~B} ; 14.0$ to $27.0 \mathrm{~min}, 100 \% \mathrm{~B} ; 27.0$ to $27.1 \mathrm{~min}, 100 \% \mathrm{~B}$ to $5 \% \mathrm{~B}$; 27.1 to $40.0 \mathrm{~min}, 5 \% \mathrm{~B}$. The flow rate was $0.4 \mathrm{~mL} / \mathrm{min}$.

The metabolite extract of each biological sample or the pooled sample was analyzed by 2DLC-MS in positive mode (+) and negative mode (-), respectively. The electrospray ionization probe was fixed at level C. The parameters for the probe were set as follows: sheath gas 55 arbitrary unit, auxiliary gas 15 arbitrary unit, sweep gas 3 arbitrary unit, spray voltage $3.5 \mathrm{kV}$, capillary temperature $320^{\circ} \mathrm{C}$, S-lens RF level 65.0, and auxiliary gas heater temperature $450{ }^{\circ} \mathrm{C}$. The method of mass spectrometer was set as follows: full scan range $50-750 \mathrm{~m} / \mathrm{z}$, resolution 30,000, maximum injection time $50 \mathrm{~ms}$, and automatic gain control (AGC) target $1 \times 10^{6}$ ions for both positive and negative modes. 
The pooled sample was also analyzed by 2DLC-MS/MS (-) and 2DLC-MS/MS (+), respectively, to acquire the MS/MS spectra of metabolites. The 2DLC-MS/MS method and electrospray ionization condition were the same as those used in parallel 2DLC-MS analyses. The parameters used for mass spectrometry were as follows: for full-MS scan, scan range 50-750 m/z, resolution 30,000, maximum injection time $50 \mathrm{~ms}$, and AGC target $1 \times 10^{6}$ ions; for dd-MS ${ }^{2}$ scan, resolution 15,000 , maximum injection time $100 \mathrm{~ms}$, AGC target $5 \times 10^{4}$ ions, loop count 6 , isolation window $1.3 \mathrm{~m} / \mathrm{z}$, and dynamic exclusion time 1.2 s. Each pooled sample was analyzed using 3 collision energies, i.e., 20, 40 and $60 \mathrm{eV}$, respectively.

To analyze the experimental data, all 2DLC-MS (-) and 2DLC-MS (+) data were processed using MetSign software for spectrum deconvolution, metabolite assignment, cross-sample peak list alignment, normalization, pattern recognition, and statistical significance test. ${ }^{125-128}$ Metabolite identification was achieved in MetSign using the 2DLCMS (-) and 2DLC-MS (+) data of biological samples and the 2DLC-MS/MS (-) and 2DLCMS/MS (+) data of the pooled sample. MetSign respectively aligned the 2DLC-MS/MS () and 2DLC-MS/MS (+) data to the 2DLC-MS (-) and 2DLC-MS (+) data by retention time and parent ion $\mathrm{m} / \mathrm{z}$ values with following thresholds: retention time variation $\leq 0.2 \mathrm{~min}$ and parent ion $\mathrm{m} / \mathrm{z}$ variation $\leq 4 \mathrm{ppm}$. To identify the metabolites in the pooled sample, the parent ion $\mathrm{m} / \mathrm{z}$, retention time, and MS/MS spectra of a metabolite were matched to the corresponding information of 205 metabolite standards recorded in an in-house database, where the matching thresholds were set as follows: MS/MS spectral similarity $\geq 0.4$, retention time difference $\leq 0.15 \mathrm{~min}$, and $\mathrm{m} / \mathrm{z}$ variation $\leq 4 \mathrm{ppm}$. The 2DLC-MS/MS (-) and 2DLC-MS/MS (+) data without a match in the in-house database were further analyzed 
using Compound Discoverer 2.0 software (Thermo Fisher Scientific, Inc., Germany) to match the remaining MS/MS spectra to the MS/MS spectra recorded in the Compound Discoverer database with a threshold of MS/MS spectral similarity score $\geq 20$. For the peaks detected in the 2DLC-MS (-) or 2DLC-MS (+) data of biological samples that were not matched to any metabolites by MS/MS spectral matching, the $m / z$ values of those parent ions were then matched to the compounds recorded in the Human Metabolome Database (HMDB) and Kyoto Encyclopedia of Genes and Genomes (KEGG) databases. The $\mathrm{m} / \mathrm{z}$ variation window was set $\leq 4 \mathrm{ppm}$.

\subsection{Results and Discussion}

\subsubsection{Metabolite Coverage by GC×GC-MS}

Metabolite identification in analysis of $\mathrm{GC} \times \mathrm{GC}-\mathrm{MS}$ data was done using the $\mathrm{GC} \times \mathrm{GC}$ MS data of the pooled sample and biological samples by spectral similarity matching followed by retention index matching. ${ }^{106,124}$ Details of data processing parameters are listed in Table 5.1.

Table 5.1. Data processing parameters used in LECO ChromaTOF software

\begin{tabular}{ll}
\hline \multicolumn{1}{c}{ Data processing parameters used in LECO ChromaTOF } \\
\hline Baseline Offset & 1 \\
Number of Data Points Averaged for Baseline Smoothing & Auto \\
& GC-MS: 4, \\
& GC×GC- \\
Peak Width for Peak Finding & MS: 7.5 \\
Minimum Number of Apexing Masses for Peak Finding & 2 \\
Minimum SNR for Peak Finding & 10 \\
Library Search Mode & Normal, \\
Library Hits Returned per Peak & Forward \\
Minimum/Maximum Molecular Weight Allowed & 10 \\
Relative Mass Threshold for Library Searching & $29 / 850$ \\
& 10
\end{tabular}


Minimum Similarity Match Before Name is Assigned

600

Libraries Used for Searching

In-House,

Mass Used for Area/Height Calculation

NIST-14

Match Required to Combine Peaks in the Second Dimension

Unique

Second Dimension Peak Width

500

Minimum SNR for Subpeak to be Retained

0.15

Integration Approach

6

Traditional

Table 5.2 lists the numbers of detected chromatographic peaks and their identification results from the pooled sample and the rat liver samples.

Table 5.2. Numbers of detected peaks from pooled samples and biological samples by GC $\times$ GC-MS and the results of metabolite identification using different matching methods

\begin{tabular}{|c|c|c|c|c|c|}
\hline \multirow[t]{2}{*}{ Sample } & \multirow{2}{*}{$\begin{array}{l}\text { Analysis } \\
\text { ID }\end{array}$} & \multirow{2}{*}{$\begin{array}{l}\text { Chromatographic } \\
\text { peaks }\end{array}$} & \multicolumn{3}{|c|}{ Metabolite identification } \\
\hline & & & $\begin{array}{l}\text { Similarity score } \\
\text { threshold } \geq 500\end{array}$ & $\begin{array}{l}\text { After RI } \\
\text { matching }\end{array}$ & $\begin{array}{l}\text { Unique } \\
\text { metabolites }\end{array}$ \\
\hline \multirow[t]{9}{*}{$\begin{array}{l}\text { Pooled } \\
\text { sample }\end{array}$} & Inj-1 & 3280 & 992 & 911 & 476 \\
\hline & Inj-2 & 3004 & 836 & 778 & 423 \\
\hline & Inj-3 & 3443 & 842 & 778 & 404 \\
\hline & Inj-4 & 3082 & 936 & 862 & 429 \\
\hline & Inj-5 & 3029 & 927 & 864 & 450 \\
\hline & Inj-6 & 3106 & 941 & 637 & 367 \\
\hline & Inj-7 & 3210 & 1037 & 947 & 447 \\
\hline & Inj-8 & 2952 & 712 & 865 & 477 \\
\hline & Average & $3138 \pm 153$ & $903 \pm 96$ & $830 \pm 91$ & $434 \pm 35$ \\
\hline \multirow{7}{*}{$\begin{array}{l}\text { Biologica } \\
\text { 1 sample }\end{array}$} & & & & & \\
\hline & S-1 & 3389 & 1017 & 902 & 484 \\
\hline & $\mathrm{S}-2$ & 3371 & 953 & 842 & 462 \\
\hline & $\mathrm{S}-3$ & 3304 & 984 & 845 & 469 \\
\hline & S-4 & 3531 & 1003 & 918 & 507 \\
\hline & $S-5$ & 3873 & 1080 & 925 & 506 \\
\hline & S-6 & 3263 & 974 & 881 & 443 \\
\hline
\end{tabular}




\begin{tabular}{ccccc} 
S-7 & 3062 & 990 & 898 & 458 \\
S-8 & 3288 & 1075 & 954 & 480 \\
S-9 & 2559 & 750 & 693 & 399 \\
S-10 & 3508 & 961 & 859 & 443 \\
S-11 & 3154 & 964 & 846 & 413 \\
S-12 & 3463 & 1048 & 929 & 470 \\
Average & $3314 \pm 301$ & $983 \pm 82$ & $874 \pm 65$ & $461 \pm 32$ \\
\hline
\end{tabular}

Out of 13 biological samples, one sample from G1 was detected as an outlier during the peaks alignment step due to very smaller number of peaks detected compared to the other samples. We believe this was caused by the injection in GC×GC-MS. Therefore, this sample was excluded in the subsequent analysis. While about $3,138 \pm 153$ chromatographic peaks were detected in the eight injections of the pooled sample, $830 \pm 91$ metabolites were identified. Using the same set of parameters, 3,314 \pm 301 chromatographic peaks were detected from the biological samples, from which $874 \pm 65$ metabolites were identified. A metabolite might be identified from multiple distinct chromatographic peaks owing to incomplete derivatization, presence of isomers, or false identifications. After removing the redundant metabolite identifications, $434 \pm 35$ unique metabolites were identified from the pooled sample and $461 \pm 32$ unique metabolites were identified from the biological samples. In GC×GC-MS, metabolites co-eluted from the first dimension GC column might be separated by the second dimension GC column (Figure 5.1), which can increase the chance of acquiring high quality EI mass spectrum for metabolite identification. 


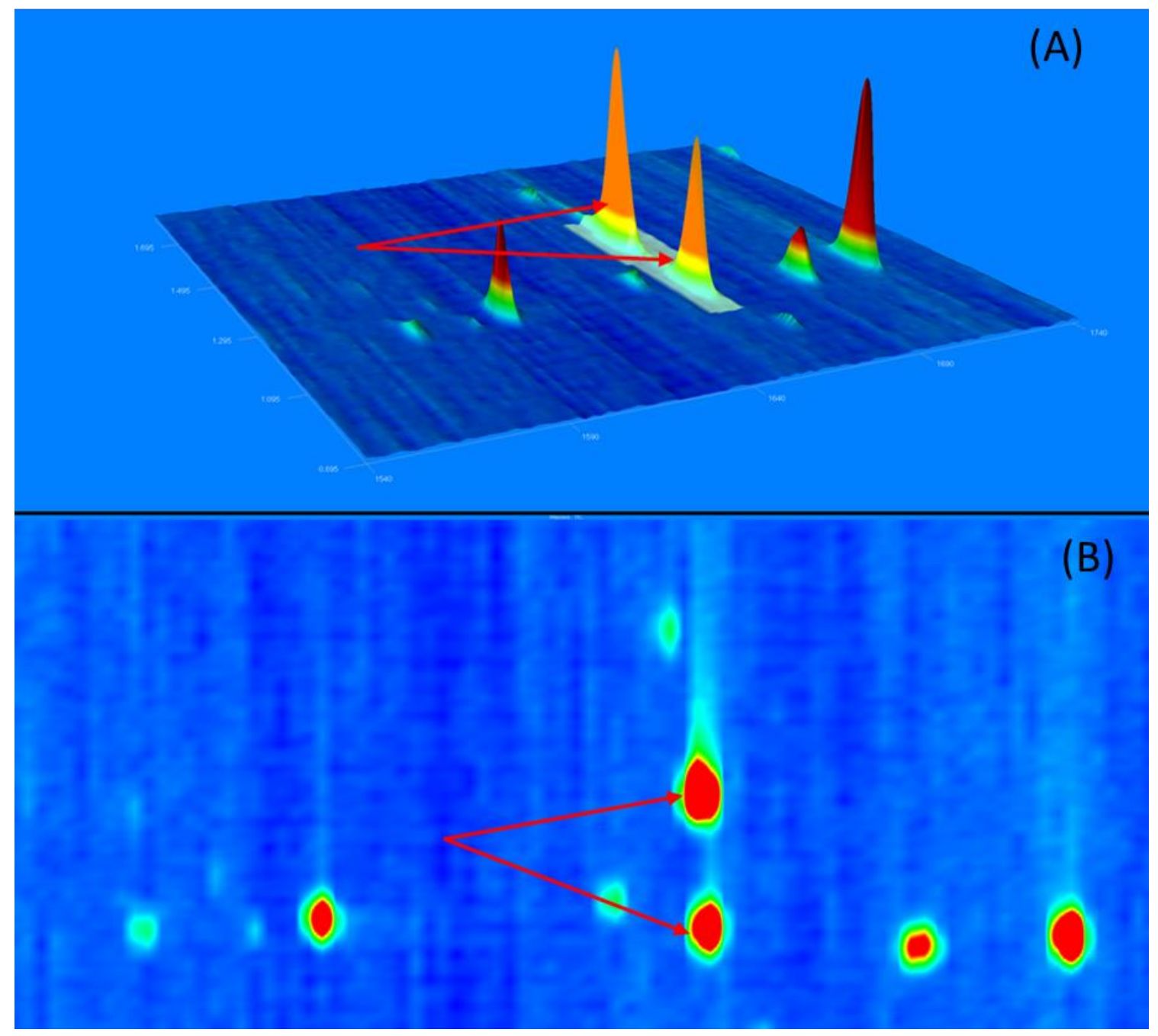

Figure 5.1. Two compounds co-eluted from the first dimension GC but separated by the second dimension GC. (A) is a three-dimensional view, and (B) is a contour plot.

Despite the excellent instrumental capability, the identification accuracy of current mass spectrum matching method is only about $79.6 \% .^{33,129,130}$ Therefore, additional information such as retention index must be used to reduce the rate of false identifications. ${ }^{131,132}$ On average, retention index matching removed about $8.9 \%$ of false identifications generated by mass spectrum matching (Table 5.2). Overall, only about 
$13.7 \%$ chromatographic peaks detected by $\mathrm{GC} \times \mathrm{GC}-\mathrm{MS}$ were assigned to metabolites in this study.

\subsubsection{Metabolite Coverage by Parallel 2DLC-MS}

To identify metabolites from the 2DLC-MS data, 2DLC-MS/MS (-) and 2DLCMS/MS (+) data of the pooled sample were first respectively aligned with the 2DLC-MS (-) and 2DLC-MS (+) data of the biological samples based on the retention time and parent ion $\mathrm{m} / \mathrm{z}$ of each metabolite. The aligned data were then used for metabolite identification. Table 5.3 summarizes the identification results of the 2DLC-MS data. We made fresh solvent of mobile phase for analysis of three samples by 2DLC-MS, one sample from G1 and two samples from G2. In order to avoid the potential problems of solvent change, the experiment data of these three samples were not included in Table 5.3. Details of metabolites identified in this study by MS/MS matching are listed in Tables 5.4 and 5.5. 
Table 5.3. Numbers of detected peaks from pooled samples and biological samples by 2DLC-MS and the results of metabolite identification using different matching methods.

\begin{tabular}{|c|c|c|c|c|c|c|c|}
\hline Sample & $\begin{array}{l}\text { Analysis } \\
\text { ID }\end{array}$ & 2DLC & MS (-) & & 2DLC- & $\operatorname{MS}(+)$ & \\
\hline & & $\begin{array}{c}\text { Isotopic } \\
\text { peaks }\end{array}$ & $\begin{array}{c}\text { Public } \\
\text { DB }\end{array}$ & MS/MS DB & $\begin{array}{c}\text { Isotopic } \\
\text { peaks }\end{array}$ & $\begin{array}{c}\text { Public } \\
\text { DB }\end{array}$ & MS/MS DB \\
\hline & Inj-1 & 15586 & 2706 & 278 & 14617 & 4241 & 217 \\
\hline & Inj-2 & 14903 & 2526 & 276 & 14834 & 4215 & 224 \\
\hline & Inj-3 & 15381 & 2494 & 275 & 14823 & 4265 & 217 \\
\hline & Inj-4 & 15196 & 2564 & 267 & 15109 & 4405 & 229 \\
\hline & Inj-5 & 14951 & 2440 & 274 & 16033 & 4590 & 231 \\
\hline & Inj-6 & 14302 & 2201 & 220 & 16238 & 4621 & 223 \\
\hline & Inj-7 & 14959 & 2313 & 255 & 16341 & 4546 & 236 \\
\hline & Inj-8 & 15585 & 2494 & 270 & 16079 & 4489 & 236 \\
\hline Pooled sample & Average & $15108 \pm 399$ & $2467 \pm 144$ & $264 \pm 18$ & $15509 \pm 681$ & $4422 \pm 153$ & $227 \pm 7$ \\
\hline & S-1 & 15112 & 3590 & 264 & 18128 & 5791 & 214 \\
\hline & S-2 & 14753 & 3363 & 266 & 17164 & 5561 & 197 \\
\hline & S-3 & 15184 & 3625 & 274 & 17875 & 5572 & 201 \\
\hline & S-4 & 14699 & 3560 & 262 & 18193 & 5503 & 205 \\
\hline & S-5 & 15075 & 3673 & 276 & 17922 & 5371 & 186 \\
\hline & S-6 & 14776 & 3514 & 278 & 17082 & 5457 & 189 \\
\hline & S-7 & 14844 & 3463 & 242 & 17806 & 5806 & 196 \\
\hline & S-8 & 15514 & 3553 & 279 & 17450 & 5519 & 217 \\
\hline & S-9 & 14888 & 3623 & 237 & 17010 & 5235 & 196 \\
\hline & S-10 & 14546 & 3550 & 243 & 17541 & 5788 & 202 \\
\hline Biological sample & Average & $14939 \pm 269$ & $3551 \pm 85$ & $262 \pm 15$ & $17617 \pm 410$ & $5560 \pm 180$ & $200 \pm 9$ \\
\hline
\end{tabular}


Table 5.4. Details of metabolites identified by MS/MS matching in 2DLC-MS (-) mode

\begin{tabular}{|c|c|c|c|}
\hline Name & $m / z$ & $\begin{array}{l}\text { Retention time } \\
(\mathrm{min})\end{array}$ & Database \\
\hline$( \pm) 13-\mathrm{HODE}$ & 295.227 & 13.96 & In-house $\mathrm{DB}^{\mathrm{a}}$ \\
\hline (R)-2-Hydroxybutyric acid & 103.04 & 2.13 & In-house DB \\
\hline 2-Hydroxycaproic acid & 131.071 & 2.45 & $\mathrm{CD}^{\mathrm{b}}$ \\
\hline 2-Methylglutaric acid & 145.051 & 2.17 & $\mathrm{CD}$ \\
\hline 2-Oxobutyric acid & 101.024 & 5.54 & $\mathrm{CD}$ \\
\hline 2-Oxoglutaric acid & 145.014 & 5.36 & $\mathrm{CD}$ \\
\hline 2-Phosphoglycerate (2PG) & 184.985 & 0.88 & In-house DB \\
\hline 3-AMP & 346.055 & 1.2 & In-house DB \\
\hline 3-Hydroxybutyric acid & 103.04 & 1.68 & In-house DB \\
\hline 3-Phosphoglyceric acid & 184.985 & 6.3 & $\mathrm{CD}$ \\
\hline 4-Dodecylbenzenesulfonic acid & 325.184 & 4.52 & $\mathrm{CD}$ \\
\hline 4-Methyl-2-oxovaleric acid & 129.056 & 7.53 & In-house DB \\
\hline 4-Oxoproline & 128.035 & 4.53 & $\mathrm{CD}$ \\
\hline 5-CMP & 322.044 & 0.94 & In-house DB \\
\hline Alanine & 88.0402 & 0.83 & In-house DB \\
\hline Alpha-D-Glucose 1-phosphate & 259.023 & 0.83 & In-house DB \\
\hline alpha ketoglutaric acid & 145.014 & 1.15 & In-house DB \\
\hline Arabitol & 151.061 & 5.16 & $\mathrm{CD}$ \\
\hline Asparagine & 131.046 & 0.83 & In-house DB \\
\hline Aspartic Acid & 132.03 & 0.83 & In-house DB \\
\hline Azelaic acid & 187.097 & 9.05 & In-house DB \\
\hline Benzoic acid & 121.029 & 0.1 & $\mathrm{CD}$ \\
\hline Citrate & 191.02 & 1.21 & In-house DB \\
\hline Citrulline & 174.088 & 0.85 & In-house DB \\
\hline Cystathionine & 221.06 & 0.8 & In-house DB \\
\hline D-Erythrose-4-phosphate & 199.001 & 0.88 & In-house DB \\
\hline D-Glucosamine-6-phosphate & 258.038 & 5.97 & In-house DB \\
\hline D-Glucose (Glc) & 179.056 & 0.88 & In-house DB \\
\hline D-Glucose 6-phosphate & 259.022 & 6.2 & $\mathrm{CD}$ \\
\hline $\begin{array}{l}\text { D-Glyceraldehyde 3-Phosphate } \\
\text { (GADP) }\end{array}$ & 168.991 & 0.91 & In-house DB \\
\hline $\begin{array}{l}\text { Dihydroxyacetone Phosphate } \\
\text { (DHAP) }\end{array}$ & 168.991 & 4.96 & In-house DB \\
\hline DL-alpha-Glycerol phosphate & 171.006 & 0.86 & In-house DB \\
\hline DL- $\beta$-Leucine & 130.087 & 4.96 & $\mathrm{CD}$ \\
\hline Dodecanedioic acid & 229.144 & 9.59 & $\mathrm{CD}$ \\
\hline D-Ribulose-5-phosphate & 229.012 & 0.85 & In-house DB \\
\hline
\end{tabular}




\begin{tabular}{|c|c|c|c|}
\hline D-Saccharic acid & 209.03 & 0.86 & $\mathrm{CD}$ \\
\hline Esculin & 339.074 & 0.89 & $\mathrm{CD}$ \\
\hline Ethyl- $\beta$-D-glucuronide & 221.067 & 1.46 & $\mathrm{CD}$ \\
\hline Fructose & 179.056 & 5.56 & $\mathrm{CD}$ \\
\hline Fumaric acid & 115.004 & 1.53 & In-house DB \\
\hline Glutamic acid & 146.046 & 0.85 & In-house DB \\
\hline Glutamine & 145.062 & 5.74 & $\mathrm{CD}$ \\
\hline Glutaric acid & 131.035 & 2.44 & In-house DB \\
\hline Glutathione & 306.076 & 1.15 & In-house DB \\
\hline Glycerol 3-phosphate & 171.006 & 5.75 & $\mathrm{CD}$ \\
\hline Glycine & 74.0246 & 0.83 & In-house DB \\
\hline Glycyl-L-leucine & 187.109 & 5.25 & $\mathrm{CD}$ \\
\hline Histidine & 154.062 & 0.79 & In-house DB \\
\hline Hypotaurine & 108.012 & 5.7 & $\mathrm{CD}$ \\
\hline Hypoxanthine & 135.031 & 1.18 & $\mathrm{CD}$ \\
\hline Inosine & 267.073 & 1.6 & In-house DB \\
\hline Isobutyric acid & 87.045 & 2.45 & In-house DB \\
\hline Isocitric acid & 191.02 & 0.89 & In-house DB \\
\hline Isoleucine & 130.087 & 1.75 & In-house DB \\
\hline Itaconic acid & 129.019 & 6.16 & $\mathrm{CD}$ \\
\hline Lactic acid & 89.0243 & 1.24 & In-house DB \\
\hline L-alpha-Aminoadipic acid & 160.061 & 0.9 & In-house DB \\
\hline L-alpha-Amino-n-butyric acid & 102.056 & 0.85 & In-house DB \\
\hline L-Ascorbic acid 2-sulfate & 254.981 & 0.97 & $\mathrm{CD}$ \\
\hline Leukotriene B4 & 335.222 & 2.42 & In-house DB \\
\hline Levulinic acid & 115.04 & 2.43 & In-house DB \\
\hline Glutathione reduced & 306.076 & 5.62 & $\mathrm{CD}$ \\
\hline L-Glutathione oxidized & 611.143 & 1.17 & $\mathrm{CD}$ \\
\hline Lipoxin A4 & 351.217 & 2.43 & In-house DB \\
\hline Lipoxin B4 & 351.217 & 10.39 & In-house DB \\
\hline Phenylalanine & 164.072 & 4.93 & $\mathrm{CD}$ \\
\hline Pyroglutamic acid & 128.035 & 1.35 & In-house DB \\
\hline Serine & 104.035 & 5.96 & $\mathrm{CD}$ \\
\hline Lysine & 145.098 & 0.75 & In-house DB \\
\hline Malic acid & 133.014 & 1.04 & In-house DB \\
\hline Maleic acid & 115.004 & 1.2 & In-house DB \\
\hline Malonic acid & 103.004 & 1.11 & In-house DB \\
\hline Maltose & 341.108 & 5.74 & $\mathrm{CD}$ \\
\hline Mannitol & 181.072 & 5.36 & $\mathrm{CD}$ \\
\hline Mesaconic acid & 129.019 & 0.89 & $\mathrm{CD}$ \\
\hline Methionine & 148.044 & 1.21 & In-house DB \\
\hline
\end{tabular}




\begin{tabular}{|c|c|c|c|}
\hline Monobutyl phthalate & 221.082 & 10.73 & $\mathrm{CD}$ \\
\hline N-Acetylalanine & 130.051 & 1.65 & $\mathrm{CD}$ \\
\hline N-Acetyl-alpha-D-glucosamine & & & \\
\hline 1-phosphate & 300.049 & 0.84 & In-house DB \\
\hline N-Acetyl-D-Glucosamine & 220.082 & 0.95 & In-house DB \\
\hline N-Acetyl-L-glutamic acid & 188.056 & 1.22 & In-house DB \\
\hline N-Acetyl-L-glutamine & 187.072 & 4.61 & $\mathrm{CD}$ \\
\hline N-Acetylneuraminic acid & 308.098 & 0.91 & $\mathrm{CD}$ \\
\hline $\begin{array}{l}\text { N-Acetyl- } \alpha \text {-D-glucosamine 1- } \\
\text { phosphate }\end{array}$ & 300.048 & 5.85 & $\mathrm{CD}$ \\
\hline Nicotinic acid & 122.025 & 1.16 & In-house DB \\
\hline $\mathrm{N}-\alpha$-Acetyl-L-asparagine & 173.057 & 5.79 & \\
\hline O-Phosphoethanolamine & 140.012 & 0.82 & In-house DB \\
\hline O-Phosphorylethanolamine & 140.012 & 6.22 & $\mathrm{CD}$ \\
\hline Ornithine & 131.082 & 0.78 & In-house DB \\
\hline Orotic acid & 155.01 & 4.85 & $\mathrm{CD}$ \\
\hline Oxalic acid & 88.9879 & 0.89 & In-house DB \\
\hline Pantothenic acid & 218.103 & 3.97 & $\mathrm{CD}$ \\
\hline Phosphoenolpyruvate & 166.975 & 0.9 & In-house DB \\
\hline Phosphoenolpyruvic acid & 166.975 & 6.03 & $\mathrm{CD}$ \\
\hline Prostaglandin G2 & 367.211 & 2.63 & In-house DB \\
\hline Pyruvate & 87.0086 & 1.07 & In-house DB \\
\hline Quinolinic Acid & 166.015 & 1.19 & In-house DB \\
\hline Ribose & 149.045 & 4.81 & $\mathrm{CD}$ \\
\hline Salicylic acid & 137.024 & 9.35 & In-house DB \\
\hline Succinate & 117.019 & 1.61 & In-house DB \\
\hline Sucrose & 341.108 & 0.89 & In-house DB \\
\hline Taurine & 124.007 & 0.85 & In-house DB \\
\hline Taurochenodeoxycholic acid & 498.289 & 9.71 & $\mathrm{CD}$ \\
\hline Thiamine & 263.097 & 0.78 & In-house DB \\
\hline Threonine & 118.051 & 0.86 & In-house DB \\
\hline trans-4-Hydroxy-L-proline & 130.051 & 0.89 & In-house DB \\
\hline trans-Ferulic acid & 193.051 & 2.43 & In-house DB \\
\hline Tryptophan & 203.083 & 5.12 & $\mathrm{CD}$ \\
\hline Tyrosine & 180.067 & 1.2 & In-house DB \\
\hline UMP & 323.028 & 1.08 & In-house DB \\
\hline Uracil & 111.02 & 1.15 & $\mathrm{CD}$ \\
\hline Uric acid & 167.021 & 1.12 & $\mathrm{CD}$ \\
\hline Uridine & 243.062 & 4.57 & $\mathrm{CD}$ \\
\hline Valine & 116.072 & 1.05 & In-house DB \\
\hline Xanthine & 151.026 & 1.32 & $\mathrm{CD}$ \\
\hline
\end{tabular}




\begin{tabular}{lrrl} 
Xanthosine & 283.068 & 4.72 & $\mathrm{CD}$ \\
$\alpha$-D-Mannose 1-phosphate & 259.022 & 6.02 & $\mathrm{CD}$ \\
$\beta$-D-Glucopyranuronic acid & 193.035 & 6.05 & $\mathrm{CD}$ \\
$\delta$-Ribono-1,4-lactone & 147.03 & 5.91 & $\mathrm{CD}$ \\
Y-Aminobutyric acid (GABA) & 102.056 & 5.91 & $\mathrm{CD}$ \\
\hline
\end{tabular}

Table 5.5. Details of metabolites identified by MS/MS matching in 2DLC-MS (+) mode

\begin{tabular}{|c|c|c|c|}
\hline Name & $m / z$ & $\begin{array}{l}\text { Retention } \\
\text { time (min) }\end{array}$ & MS/MS database \\
\hline 1-Methyl-L-histidine & 170.092 & 0.75 & In-house DBa \\
\hline 2-Aminobutyric acid & 104.07 & 5.39 & $\mathrm{CDb}$ \\
\hline 3-Aminobutanoic acid & 104.07 & 0.87 & In-house DB \\
\hline 3-Hydroxypyridine & 96.0442 & 0.93 & In-house DB \\
\hline 4-Acetamidobutanoic acid & 146.081 & 5.38 & $\mathrm{CD}$ \\
\hline 4-Guanidinobutyric acid & 146.092 & 1.03 & $\mathrm{CD}$ \\
\hline 4-Indolecarbaldehyde & 146.06 & 5.08 & $\mathrm{CD}$ \\
\hline 4-Methyl-5-thiazoleethanol & 144.048 & 6.18 & $\mathrm{CD}$ \\
\hline 5-Aminovaleric acid & 118.086 & 0.88 & In-house DB \\
\hline 5-Methylcytosine & 126.066 & 1.19 & $\mathrm{CD}$ \\
\hline Alanine & 90.0548 & 0.84 & In-house DB \\
\hline alpha-Aminoadipic acid & 162.076 & 0.93 & In-house DB \\
\hline Arginine & 175.119 & 7.18 & $\mathrm{CD}$ \\
\hline Asparagine & 133.061 & 5.84 & $\mathrm{CD}$ \\
\hline Aspartic acid & 134.045 & 6.23 & $\mathrm{CD}$ \\
\hline Betaine & 118.086 & 5.06 & $\mathrm{CD}$ \\
\hline Carnitine & 162.112 & 4.31 & $\mathrm{CD}$ \\
\hline Choline & 104.107 & 0.83 & $\mathrm{CD}$ \\
\hline cis-4-Hydroxy-D-proline & 132.066 & 5.57 & $\mathrm{CD}$ \\
\hline Citrulline & 176.103 & 0.85 & In-house DB \\
\hline Creatine & 132.077 & 5.85 & $\mathrm{CD}$ \\
\hline Cystathionine & 223.074 & 0.81 & In-house DB \\
\hline Cystine & 241.03 & 6.29 & $\mathrm{CD}$ \\
\hline Cytosine & 112.05 & 6.22 & $\mathrm{CD}$ \\
\hline D-Serine & 106.05 & 6.02 & $\mathrm{CD}$ \\
\hline Ethanolamine & 62.0601 & 0.8 & In-house DB \\
\hline Glucose 1-phosphate & 261.036 & 6.28 & $\mathrm{CD}$ \\
\hline Glutamic acid & 148.06 & 5.88 & $\mathrm{CD}$ \\
\hline Glutamine & 147.076 & 0.86 & In-house DB \\
\hline
\end{tabular}




\begin{tabular}{|c|c|c|c|}
\hline Glycine & 76.0392 & 0.81 & In-house DB \\
\hline Histidine & 156.077 & 0.79 & In-house DB \\
\hline Homoserine & 120.066 & 5.74 & $\mathrm{CD}$ \\
\hline Hypotaurine & 110.027 & 5.72 & $\mathrm{CD}$ \\
\hline Hypoxanthine & 137.046 & 4.62 & $\mathrm{CD}$ \\
\hline Isoleucine & 132.102 & 1.75 & In-house DB \\
\hline Leucine & 132.102 & 1.87 & In-house DB \\
\hline Leucylproline & 229.154 & 4.81 & $\mathrm{CD}$ \\
\hline Lysine & 147.113 & 7.04 & CD \\
\hline Methionine & 150.058 & 1.2 & In-house DB \\
\hline Methylimidazoleacetic acid & 141.066 & 5.74 & $\mathrm{CD}$ \\
\hline N3,N4-Dimethyl-L-arginine & 203.15 & 6.15 & $\mathrm{CD}$ \\
\hline N6,N6,N6-Trimethyl-L-lysine & 189.16 & 6.31 & $\mathrm{CD}$ \\
\hline N6-Acetyl-L-lysine & 189.123 & 1.02 & $\mathrm{CD}$ \\
\hline N-Acetyl-D-galactosamine & 222.097 & 5.59 & CD \\
\hline N-Acetyl-D-Glucosamine & 222.097 & 0.92 & In-house DB \\
\hline N-Acetyl-L-glutamic acid & 190.071 & 1.44 & In-house DB \\
\hline N-Acetyl- $\alpha$-D-glucosamine & 222.097 & 5.23 & $\mathrm{CD}$ \\
\hline Nicotinamide & 123.055 & 1.18 & $\mathrm{CD}$ \\
\hline Nicotinic acid & 124.039 & 1.17 & In-house DB \\
\hline O-Phosphoethanolamine & 142.026 & 0.82 & In-house DB \\
\hline Ornithine & 133.097 & 0.75 & In-house DB \\
\hline Phenylalanine & 166.086 & 3 & In-house DB \\
\hline Phosphoenolpyruvate & 168.989 & 0.92 & In-house DB \\
\hline Pipecolinic acid & 130.086 & 0.75 & $\mathrm{CD}$ \\
\hline Proline & 116.07 & 5.31 & $\mathrm{CD}$ \\
\hline Pyridoxal & 168.065 & 0.92 & $\mathrm{CD}$ \\
\hline Pyroglutamic acid & 130.05 & 5.74 & $\mathrm{CD}$ \\
\hline Serine & 106.05 & 0.83 & In-house DB \\
\hline Stachydrine & 144.102 & 0.91 & $\mathrm{CD}$ \\
\hline Taurine & 126.022 & 5.61 & $\mathrm{CD}$ \\
\hline Threonine & 120.065 & 0.85 & In-house DB \\
\hline Tryptophan & 205.097 & 5.08 & $\mathrm{CD}$ \\
\hline Tyrosine & 182.081 & 1.44 & In-house DB \\
\hline Uracil & 113.034 & 4.53 & $\mathrm{CD}$ \\
\hline Urea & 61.0397 & 0.92 & In-house DB \\
\hline Uric acid & 169.035 & 5.28 & $\mathrm{CD}$ \\
\hline Urocanic acid & 139.05 & 1.03 & $\mathrm{CD}$ \\
\hline Valine & 118.086 & 4.33 & In-house DB \\
\hline $\begin{array}{l}\text { Y-Aminobutyric acid } \\
\text { (GABA) }\end{array}$ & 104.07 & 6.11 & $\mathrm{CD}$ \\
\hline
\end{tabular}


A total of $15,108 \pm 399$ and $15,509 \pm 681$ features were detected from the pooled sample by 2DLC-MS (-) and 2DLC-MS (+), respectively. Here, a feature in 2DLC-MS data was defined by retention time and isotopic peak $m / z$ value. By parent ion $m / z$ matching, $2,467 \pm 144$ features in 2DLC-MS (-) and 4,422 \pm 153 features in 2DLC-MS (+) of the pooled sample were assigned to at least one metabolite in KEGG or HMDB databases. By MS/MS spectrum matching, $264 \pm 18$ metabolites were identified from the 2DLC-MS/MS (-) data and $227 \pm 7$ metabolites were identified from the 2DLC-MS/MS (+) data.

Table 5.3 also shows that $14,939 \pm 269$ and 17,617 \pm 410 features were detected from the biological samples by 2DLC-MS (-) and 2DLC-MS (+), respectively. Among those features, 3,551 \pm 85 and 5,560 \pm 180 metabolites were respectively assigned to the 2DLCMS (-) and 2DLC-MS (+) data based on parent ion $\mathrm{m} / \mathrm{z}$ matching. By MS/MS spectrum matching, $262 \pm 15$ and $200 \pm 9$ metabolites were identified from the 2DLC-MS/MS (-) and 2DLC-MS/MS (+), respectively.

By parent ion $\mathrm{m} / \mathrm{z}$ matching, about $23.8 \%$ to $31.6 \%$ of metabolites giving rise to the features in 2DLC-MS (-) and 2DLC-MS (+) were assigned to at least one metabolite. However, the percentage of assignment was dramatically reduced to only $1.1 \%$ to $1.8 \%$ when the MS/MS spectra were used for metabolite identification, even though we maximized the chance of acquiring high quality MS/MS spectra for each metabolite by fragmenting each parent ion using three collision energies, i.e., 20, 40 and $60 \mathrm{eV}$, respectively. The extremely low percentage of metabolite identification agrees with Silva, et al.'s observation that $98 \%$ of the instrumental data were not used in metabolomics. ${ }^{133}$ Multiple factors contributed to those results. For instance, a fraction of the isotopic peaks detected by MS are not monoisotopic peaks and therefore cannot be matched to the 
metabolites. During the experiment, the top six abundant ions were subjected to MS/MS data acquisition in the dd-MS ${ }^{2}$ mode. A number of metabolites with low instrument response were not selected for MS/MS spectra acquisition even though they were detected in the full MS mode. Furthermore, a metabolite in the sample might not be present in our in-house database and the Compound Discoverer database, and therefore could not have an identification result by MS/MS spectrum matching.

\subsubsection{Integrating GC×GC-MS and Parallel 2DLC-MS Data for Higher Metabolite}

\section{Coverage}

Figure 5.2 (A) depicts the identification results of the three analytical platforms GC $\times$ GC-MS, 2DLC-MS (-), and 2DLC-MS (+) in analyzing the polar metabolites extracted from the biological samples. A total of 3,965 peaks were assigned to metabolites from the GC×GC-MS, 2DLC-MS (-), and 2DLC-MS (+) data.

A.

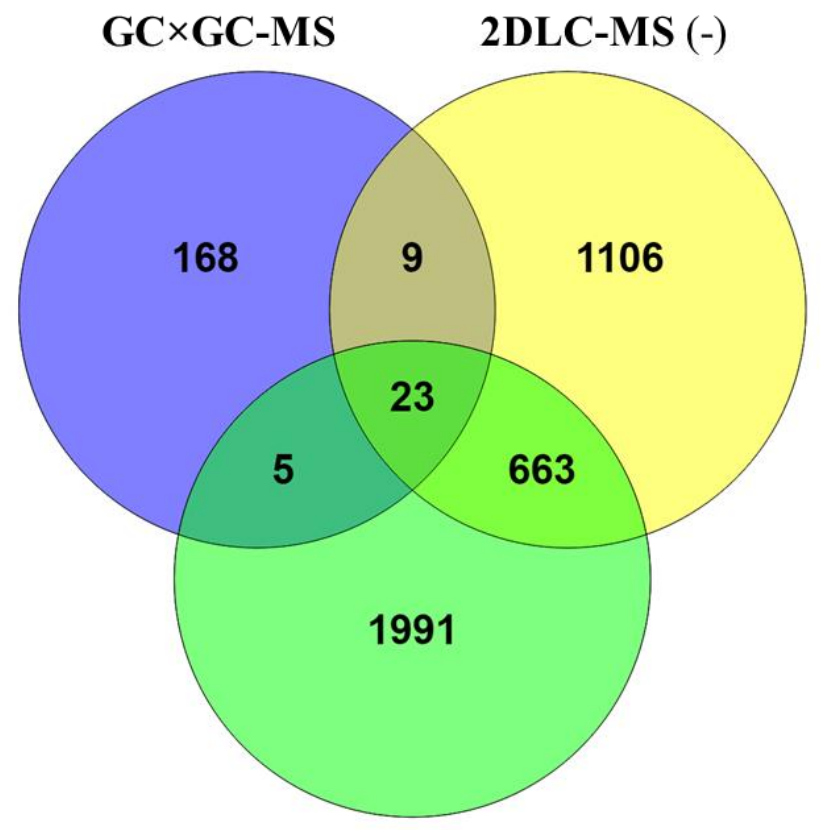

2DLC-MS (+) 
B.

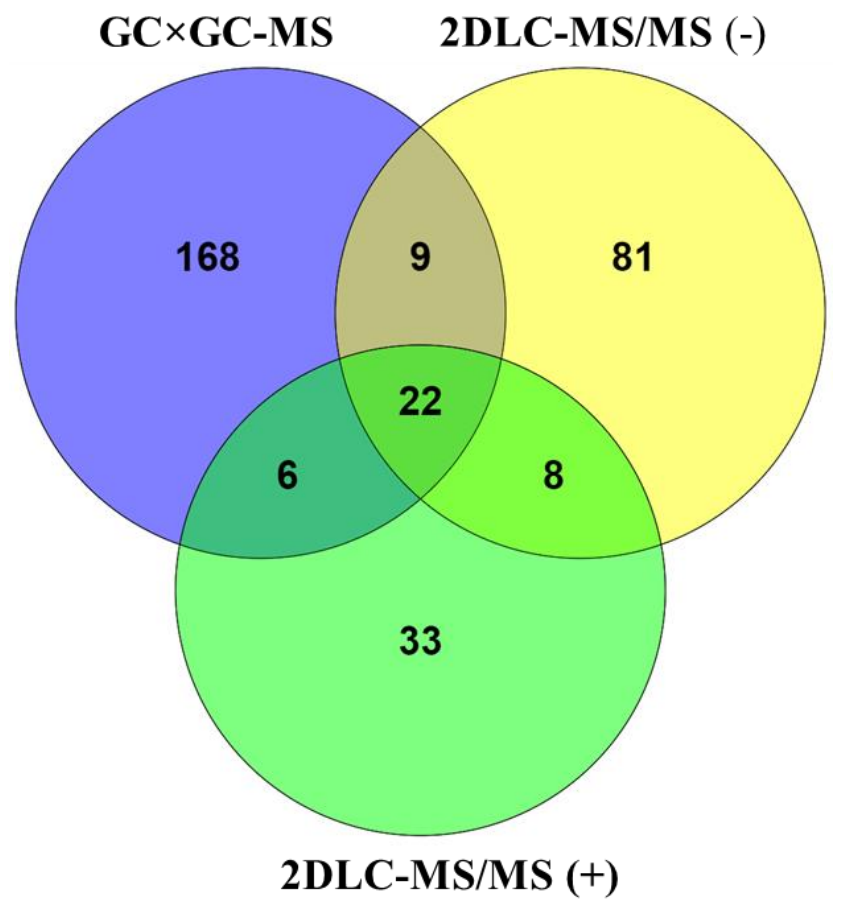

Figure 5.2. Overlap of metabolite identification. Metabolites were identified from GC $\times$ GC-MS by EI mass spectrum matching and retention index matching. (A) The metabolite identification in the analysis of 2DLC-MS data was done by parent ion $\mathrm{m} / \mathrm{z}$ matching. (B) The metabolite identification in the analysis of 2DLC-MS/MS data by MS/MS spectrum matching with or without retention time match.

A majority of metabolites were assigned by parent ion $\mathrm{m} / \mathrm{z}$ in the 2DLC-MS (-) and 2DLC-MS (+) data, while all metabolites were identified from the GC $\times$ GC-MS data by mass spectrum matching. Therefore, the number of metabolites assigned using the 2DLCMS (-) and 2DLC-MS (+) data are much larger than that using the GC $\times$ GC-MS data. Owing to the diverse chemical properties of the GC and LC columns, each analytical 
platform favors detection of different metabolites in the biological samples. As expected, the GC $\times$ GC-MS and 2DLC-MS have much different metabolite coverage. Only 37 metabolites were commonly assigned to the GC×GC-MS and 2DLC-MS data. Of those 37 metabolites, 32 metabolites were assigned to the GC $\times$ GC-MS and the 2DLC-MS (-) data, and 28 metabolites were assigned to the GC $\times$ GC-MS and 2DLC-MS (+) data. There were only 23 metabolites detected by all three analytical platforms.

Assigning a metabolite to a peak by parent ion $\mathrm{m} / \mathrm{z}$ only in the analysis of 2DLC-MS data generates a very high rate of false assignments. Figure 5.2(B) depicts the overlap of metabolite identification among GC×GC-MS, 2DLC-MS/MS (-), and 2DLC-MS/MS (+) data by MS/MS spectrum matching and other constrains, i.e., retention index filtering in analysis of GC $\times$ GC-MS data, parent ion $\mathrm{m} / z$ matching in analysis of 2DLC-MS/MS (-) and 2DLC-MS/MS (+) data. A total of 326 metabolites were identified by the three platforms, of which 205, 120 and 69 metabolites were identified from the GC×GC-MS, 2DLCMS/MS (-) and 2DLC-MS/MS (+) data, respectively. Only 22 metabolites were commonly identified in all three platforms, which is only $7.0 \%$ of the total metabolites identified by the three platforms.

In this study, we employed a parallel 2DLC-MS platform that was configured with a HILIC column and a RPC column. The downside of such a configuration is that the metabolite coverage was not dramatically increased. Furthermore, one metabolite might be detected twice and therefore increased the chance of metabolite overlapping in mass spectrometry. However, the parallel 2DLC-MS configuration does not have the problem of metabolite partition between two or more fractions that occurs in the comprehensive 2DLC configuration. In addition, we can use long columns and long gradient times to 
improve the separation. Overall, each platform, i.e., GC $\times$ GC-MS, 2DLC-MS/MS (-) or 2DLC-MS/MS (+), has limited metabolite coverage. The number of metabolites commonly detected by all those platforms is very small. Therefore, it is necessary to analyze the biological samples on different platforms to increase metabolite coverage.

\subsubsection{Integrating GC $\times$ GC-MS and Parallel 2DLC-MS for Accurate Metabolite Quantification}

During the metabolite identification, metabolites identified by parent ion $\mathrm{m} / \mathrm{z}$ matching have a high ratio of false identifications compared with the identification by MS/MS spectrum matching. In order to ensure the high degree of confidence in biomarker discovery, only metabolites identified by MS/MS spectrum matching were used for the

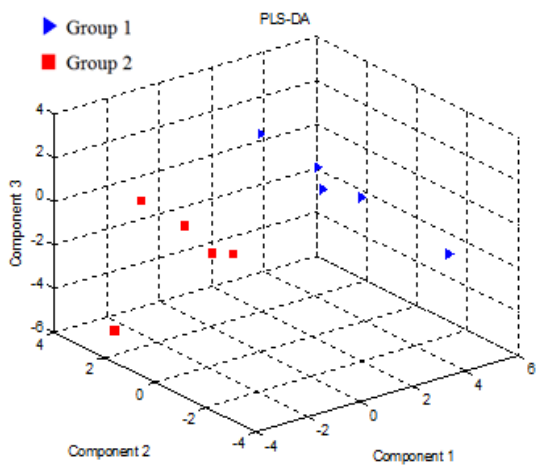

(A)

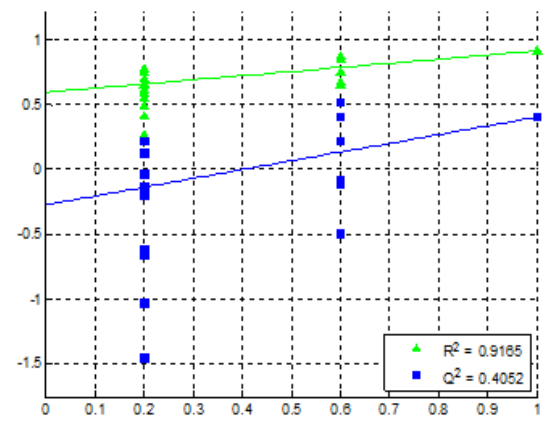

(C)

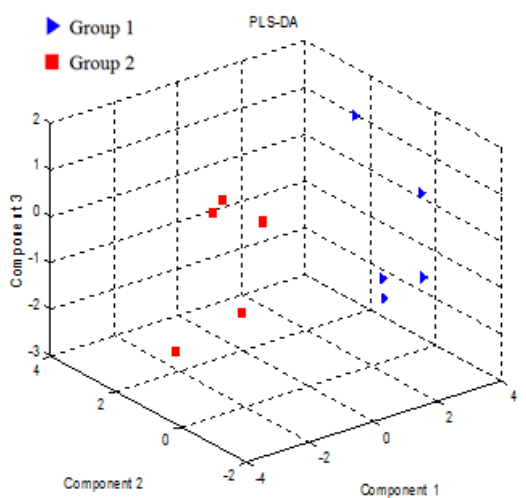

(B)

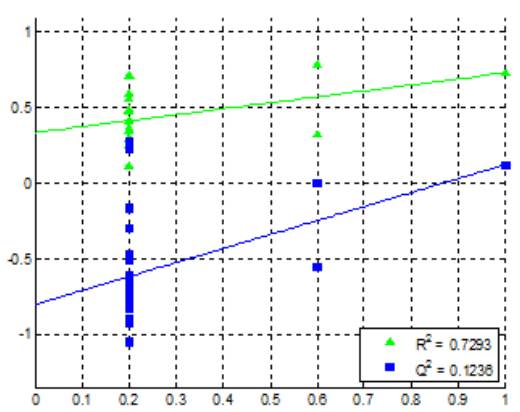

(D)

metabolite quantification. 
Figure 5.3. PLS-DA analysis of the metabolite profiles of all samples. (A) is the clustering result of 2DLC-MS (-) data. (B) is the clustering result of 2DLC-MS(+) data. (C) is the cross validation result of the PLSDA for analysis of 2DLC-MS (-) data. The magnitudes of $\mathrm{R}^{2}$ and $\mathrm{Q}^{2}$ are $\left(\mathrm{R}^{2}=0.91 \mathrm{Q}^{2}=0.40\right)$.(D) is the cross validation results of the PLSDA for analysis of 2DLC-MS (+) data with $\mathrm{R}^{2}=0.72$ and $\mathrm{Q}^{2}=0.12$.

Figure 5.3 shows clustering results of the metabolite profiles of all samples (G1 and G2) analyzed on 2DLC-MS (-) and (+) using partial least-squares discriminant analysis (PLS-DA). The two group shows significant difference in metabolite profile (Figure 5.3(A) and 5.3(B)). The cross validation also shows a good predictive ability of the PLS-DA models, with $\mathrm{Q}^{2}$ values of 0.40 for $2 \mathrm{DLC}-\mathrm{MS}(-)$ data and 0.12 for $2 \mathrm{DLC}-\mathrm{MS}(+)$ data (Figures 5.3(C) and 5.3(D)).

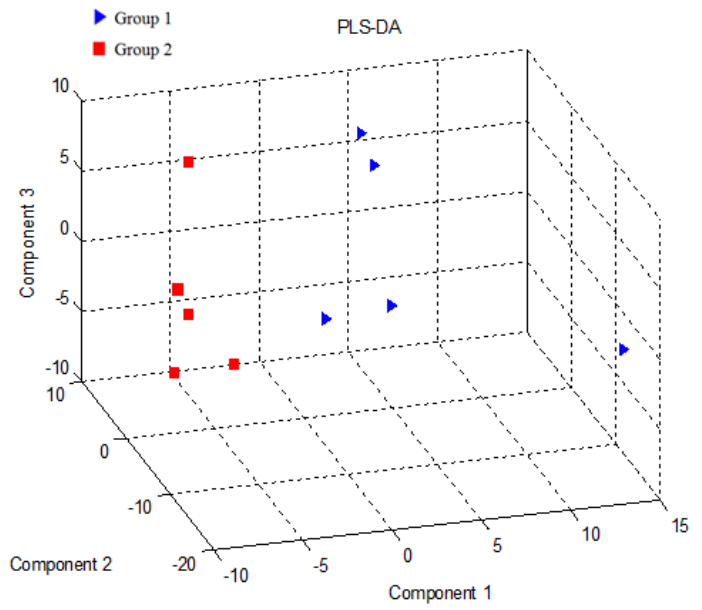

(A)

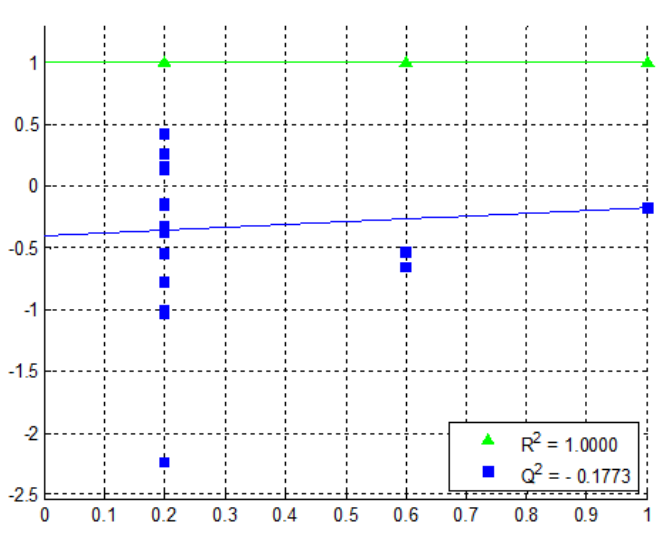

(B) 
Figure 5.4. PLSDA analysis of the metabolite profiles of all samples. (A) is the clustering result of $\mathrm{GC} \times \mathrm{GC}-\mathrm{MS}$ data. (B) is the cross validation results of the PLSDA for analysis of GC $\times$ GC-MS (+) data with $\mathrm{R}^{2}=1.0$ and $\mathrm{Q}^{2}=-0.18$.

Figure 5.4 shows clustering results of the metabolite profiles of all samples (G1 and G2) analyzed on GC $\times$ GC-MS using PLS-DA. Compared to the 2DLC-MS, GC $\times$ GC-MS data show a certain degree of separation between the G1 and the G2 with a poor predictive ability of the PLSDA models where $\mathrm{Q}^{2}$ value is -0.18 .

A pairwise two-tail $t$-test with equal variance was used to study the abundance change of each metabolite between G1 and G2, during which sample labels were permutated up to 1000 times. Table 5.6 shows that 41, 13 and 11 metabolites were detected with significant changes in their abundance levels between G1 and G2 in the GC×GC-MS, 2DLC-MS (-) and 2DLC-MS (+) data, respectively.

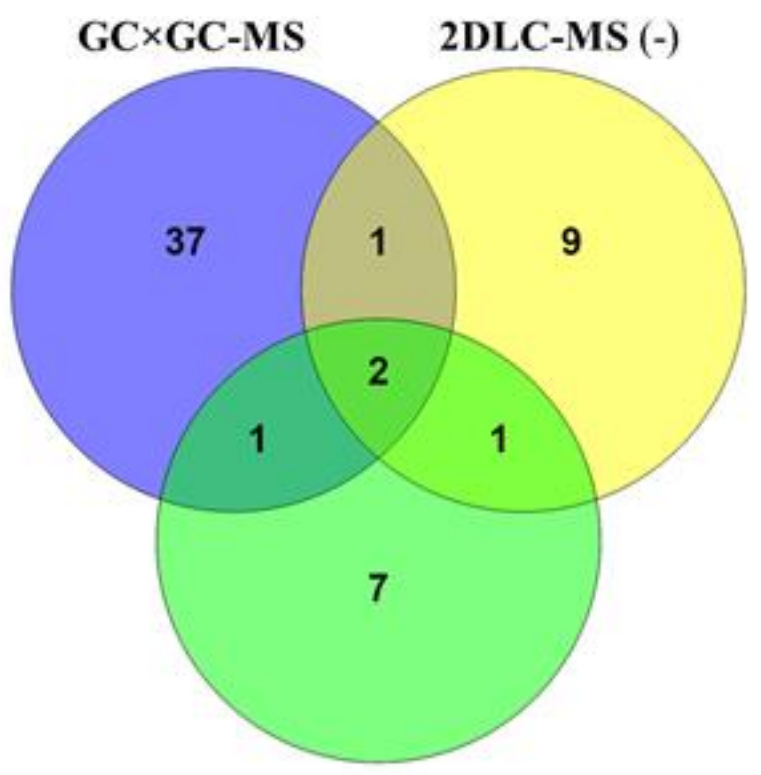

2DLC-MS (+) 
Figure 5.5. Overlap of metabolites detected with significant changes in their abundance levels between groups by three platforms.

Figure 5.5 shows the overlap of those metabolites. It is clear that each platform detected different sets of metabolites that had significant changes in their abundance levels. Among those metabolites, two were detected by all three platforms and three were detected by two of the three platforms. Integrating the results of the three platforms generated a comprehensive set of metabolites, i.e., 58 metabolites.

Table 5.6. Metabolites in rat liver detected with significant changes in their abundance levels between $\mathrm{G} 1$ and $\mathrm{G} 2$ by different platforms

\begin{tabular}{clll}
\hline Metabolite ID & \multicolumn{1}{c}{ Metabolite name } & $p$-value & Fold-change \\
& & & \\
\hline GC $\times$ GC-MS & & $4.30 \mathrm{E}-04$ & 0.8 \\
\hline 1 & 1,2-Benzisothiazol-3-amine & $2.20 \mathrm{E}-02$ & 0.9 \\
3 & 1-Butanol & $7.40 \mathrm{E}-04$ & 0.5 \\
4 & 2-Pyrrolidone-5-carboxylic acid & $1.90 \mathrm{E}-02$ & 0.6 \\
& 3-Aminobutyric acid & & \\
5 & 3-Hydroxy-3-phenylpropionic & $1.50 \mathrm{E}-02$ & 1.4 \\
6 & acid & $2.50 \mathrm{E}-02$ & 0.5 \\
7 & 3-Hydroxybutyric acid & $2.60 \mathrm{E}-02$ & 0.7 \\
8 & 5-chloroindoline-2,3-dione & $3.00 \mathrm{E}-02$ & 0.7 \\
9 & 5-Hexen-1-ol & $3.50 \mathrm{E}-02$ & 0.6 \\
10 & Acetimidamide & $9.30 \mathrm{E}-03$ & 1.9 \\
11 & Alanylglycine & $2.70 \mathrm{E}-02$ & 1.3 \\
12 & Benzoic Acid & $1.20 \mathrm{E}-02$ & 1.8 \\
13 & cis-11,14-Eicosadienoic acid & $1.00 \mathrm{E}-02$ & 0.1 \\
14 & DL-Glyceraldehyde & $5.50 \mathrm{E}-04$ & 1.4 \\
15 & Doconexent & $2.10 \mathrm{E}-02$ & 2.2 \\
16 & Eicosapentaenoic Acid & $3.80 \mathrm{E}-03$ & 0.5 \\
17 & Ethanolamine & $3.40 \mathrm{E}-04$ & 1.8 \\
18 & Heptadecanoic acid & $8.60 \mathrm{E}-04$ & 0.7 \\
19 & Hypoxanthine & $9.10 \mathrm{E}-05$ & 0.7 \\
20 & Isobutyl alcohol & $5.40 \mathrm{E}-04$ & 0.5 \\
& 1-Aspartic acid & &
\end{tabular}




\begin{tabular}{|c|c|c|c|}
\hline 21 & 1-Glutamine & $4.50 \mathrm{E}-02$ & 0.6 \\
\hline 22 & 1 -Leucine & $4.50 \mathrm{E}-02$ & 0.7 \\
\hline 23 & 1-Lysine & $8.50 \mathrm{E}-03$ & 2 \\
\hline 24 & 1-Malic acid & $5.00 \mathrm{E}-02$ & 1.6 \\
\hline 25 & 1-Ornithine & 4.10E-02 & 0.6 \\
\hline 26 & 1-Phenylalanine & 2.80E-02 & 0.6 \\
\hline 27 & 1-Pyroglutamic acid & $2.90 \mathrm{E}-02$ & 0.7 \\
\hline 28 & 1-Threonine & $7.30 \mathrm{E}-05$ & 0.4 \\
\hline 29 & Maleic acid & 4.10E-03 & 1.5 \\
\hline 30 & Methylmalonic acid & $5.70 \mathrm{E}-03$ & 0.7 \\
\hline 31 & Niacinamide & $3.20 \mathrm{E}-03$ & 0.7 \\
\hline 32 & Nonanoic acid & $1.20 \mathrm{E}-04$ & 2 \\
\hline 33 & Octadeca-9,12-diynoic acid & $9.80 \mathrm{E}-03$ & 2.6 \\
\hline 34 & O-phosphoethanolamine & $1.60 \mathrm{E}-03$ & 0.5 \\
\hline 35 & Oxalic acid & $7.90 \mathrm{E}-03$ & 2.2 \\
\hline 36 & Propylene glycol & $1.60 \mathrm{E}-02$ & 1.7 \\
\hline 37 & Pyruvate & $1.30 \mathrm{E}-02$ & 0.5 \\
\hline 38 & Stearic acid & $9.70 \mathrm{E}-04$ & 1.3 \\
\hline 39 & Taurine & 4.60E-04 & 5.6 \\
\hline 40 & Uracil & 4.40E-02 & 1.2 \\
\hline 41 & $\alpha$-Aminobutyric acid & $5.60 \mathrm{E}-04$ & 0.4 \\
\hline \multicolumn{4}{|c|}{ 2DLC-MS (-) } \\
\hline 1 & 2-Methylglutaric acid & $8.70 \mathrm{E}-03$ & 2.3 \\
\hline 2 & 4-Methyl-2-oxovaleric acid & $3.10 \mathrm{E}-02$ & 0.7 \\
\hline 3 & 4-Oxoproline & $3.90 \mathrm{E}-05$ & 0.6 \\
\hline 4 & 5-CMP & $4.00 \mathrm{E}-02$ & 1.4 \\
\hline 5 & Hypotaurine & $1.40 \mathrm{E}-03$ & 2.2 \\
\hline 6 & 1-Glutathione oxidized & $1.20 \mathrm{E}-04$ & 2 \\
\hline 7 & 1 -Malic acid & $4.90 \mathrm{E}-02$ & 1.3 \\
\hline 8 & 1-Ornithine & 4.10E-02 & 0.7 \\
\hline 9 & $\mathrm{~N}$-acetylalanine & $1.10 \mathrm{E}-02$ & 1.5 \\
\hline 10 & Pyruvic acid & $4.70 \mathrm{E}-02$ & 0.5 \\
\hline 11 & Taurine & $1.70 \mathrm{E}-03$ & 1.8 \\
\hline 12 & Taurochenodeoxycholic acid & $7.80 \mathrm{E}-05$ & 8.4 \\
\hline 13 & $\alpha$-Ketoglutaric acid & $9.80 \mathrm{E}-03$ & 0.4 \\
\hline \multicolumn{4}{|c|}{ 2DLC-MS (+) } \\
\hline 1 & 4-Acetamidobutanoic acid & $1.10 \mathrm{E}-02$ & 1.4 \\
\hline 2 & 4-Guanidinobutyric acid & $6.50 \mathrm{E}-05$ & 2.9 \\
\hline 3 & Carnitine & $1.00 \mathrm{E}-02$ & 0.8 \\
\hline 4 & Cytosine & 4.70E-02 & 10.9 \\
\hline 5 & Hypotaurine & $1.60 \mathrm{E}-03$ & 2.3 \\
\hline
\end{tabular}




\begin{tabular}{clll}
6 & 1-Ornithine & $3.20 \mathrm{E}-02$ & 0.9 \\
7 & 1-Phenylalanine & $2.40 \mathrm{E}-02$ & 0.7 \\
8 & N-acetyl-D-Glucosamine & $4.50 \mathrm{E}-02$ & 1.5 \\
9 & Proline & $9.20 \mathrm{E}-03$ & 0.9 \\
10 & Serine & $1.80 \mathrm{E}-02$ & 0.9 \\
11 & Taurine & $3.20 \mathrm{E}-02$ & 1.7 \\
\hline
\end{tabular}

Table 5.7 and Table 5.8 respectively lists the details of the abundance information of those five metabolites that were detected by more than one platform. The regulation directions of those metabolites detected in different platforms agree to each other, i.e., the fold-change of a metabolite detected in different platforms are all either larger than 1.0 or less than 1.0 (Tables 5.7 and 5.8 and Figure 5.6). Furthermore, except taurine, the foldchanges of those metabolites are almost identical, indicating the robustness and accuracy of the three platforms.
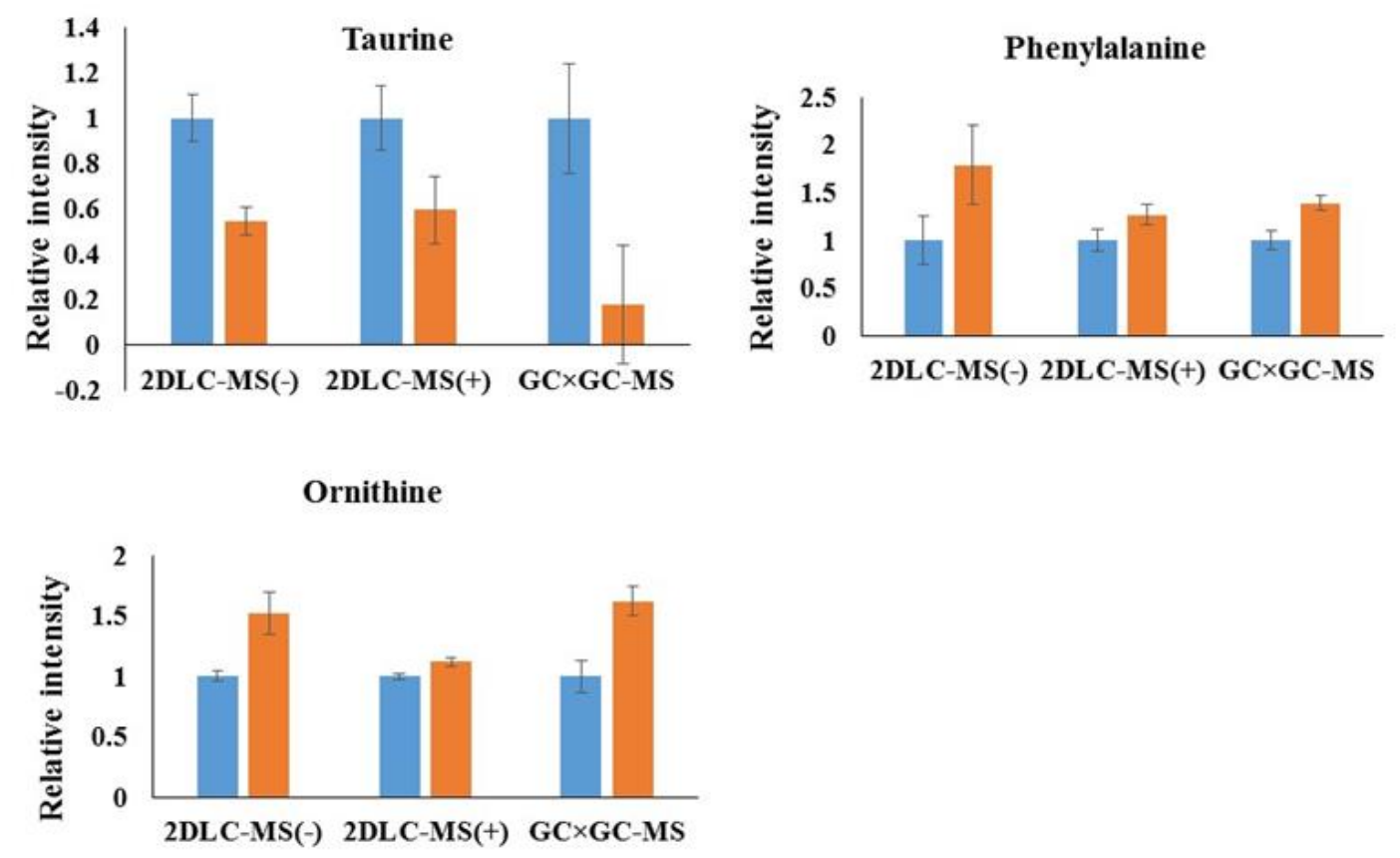
Figure 5.6. The regulation directions of metabolites that showed significant changes in their abundance levels between groups in all platforms (GC $\times$ GC-MS, 2DLC-MS (-) and 2DLCMS (+).

Table 5.7. Quantification information ( $p$-value) of metabolites that were detected by more than one platform with significant changes in their abundance levels between groups

\begin{tabular}{cccc}
\hline Compound & $p$-value & & \\
\hline & GC×GC-MS & 2DLC-MS(-) & 2DLC-MS(+) \\
\cline { 2 - 4 } Taurine & $4.60 \mathrm{E}-04$ & $1.70 \mathrm{E}-03$ & $3.20 \mathrm{E}-02$ \\
Ornithine & $4.10 \mathrm{E}-02$ & $4.10 \mathrm{E}-02$ & $3.20 \mathrm{E}-02$ \\
Phenylalanine & $2.80 \mathrm{E}-02$ & $5.70 \mathrm{E}-01$ & $2.40 \mathrm{E}-02$ \\
Malic acid & $5.00 \mathrm{E}-02$ & $4.90 \mathrm{E}-02$ & - \\
Hypotaurine & - & $1.40 \mathrm{E}-03$ & $1.60 \mathrm{E}-03$ \\
\hline
\end{tabular}

Table 5.8. Quantification information (Fold change) of metabolites that were detected by more than one platform with significant changes in their abundance levels between groups

\begin{tabular}{lccc}
\hline Compound & Fold-change & & \\
\hline & & & \\
& GC $\times$ GC-MS & 2DLC-MS(-) & 2DLC-MS(+) \\
Taurine & & & 1.7 \\
Ornithine & 5.6 & 1.8 & 0.9 \\
Phenylalanine & 0.6 & 0.7 & 0.7 \\
Malic acid & 0.6 & 0.6 & - \\
Hypotaurine & 1.6 & 1.3 & 2.3 \\
\hline
\end{tabular}

Figures 5.6(A), 5.6(B), and 5.6(C) depict the detection of L-ornithine in different platforms. This metabolite had relatively large instrument response and good 
chromatographic peak shape in all three platforms, and its chromatographic peak did not overlap with other compounds. Therefore, the quantification results of this metabolites were almost identical among the three platforms, with fold-changes of $0.6,0.7$ and 0.9 in GC $\times$ GC-MS, 2DLC-MS (-) and 2DLC-MS (+), respectively. However, the fold-change of taurine detected by GC $\times \mathrm{GC}-\mathrm{MS}$ had a larger variation compared to those detected by 2DLC-MS (-) and 2DLC-MS (+). Figures 5.7E and 5.7F show that taurine had a good instrument response and good peak shape in 2DLC-MS (-) and 2DLC-MS (+), respectively.
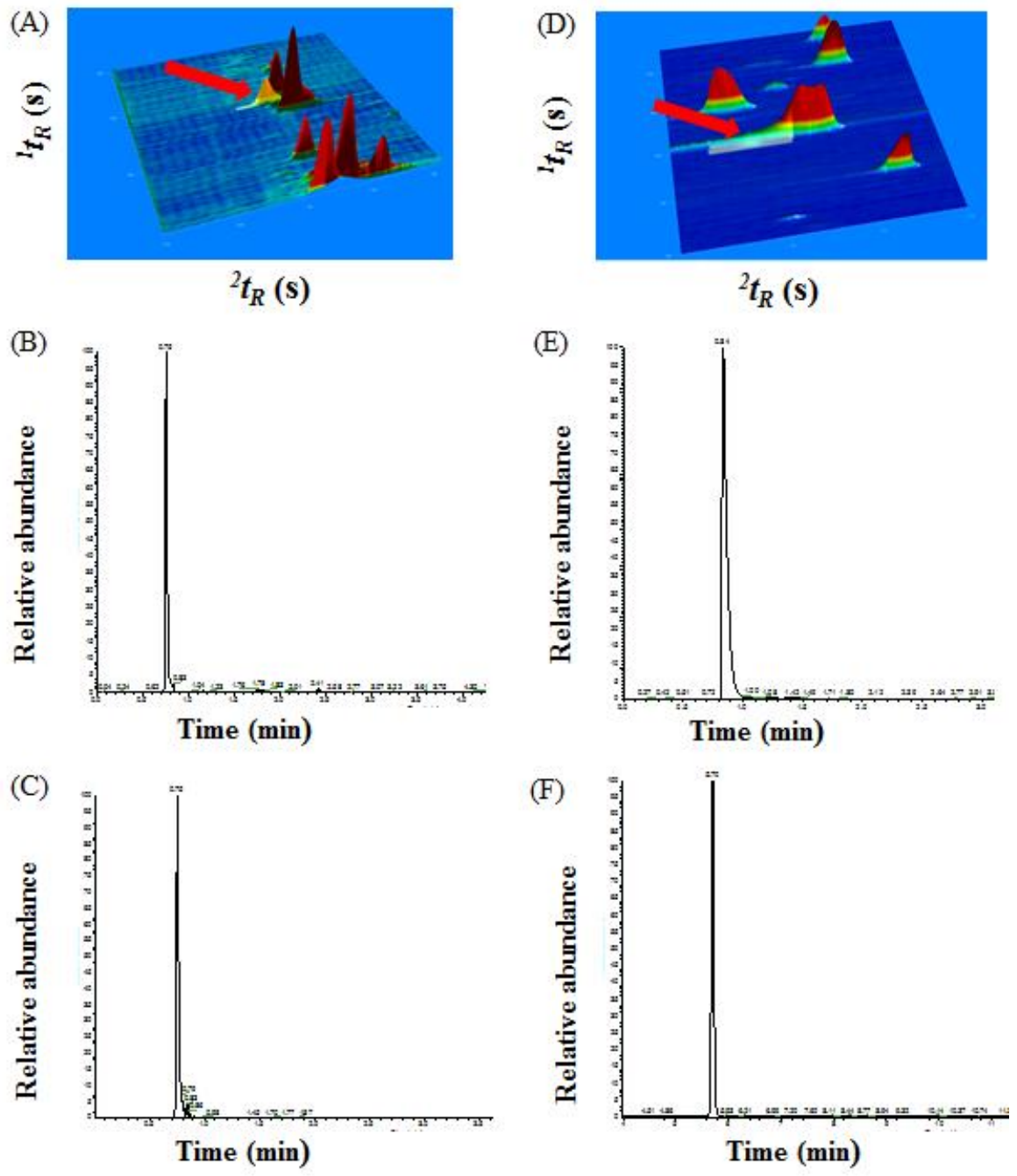
Figure 5.7. Samples of instrument response of a metabolite affecting the quantification accuracy of that metabolite. (A) Three-dimensional chromatographic peak of 1-ornithine detected by GC $\times$ GC-MS. (B) Extracted ion chromatograms of 1-ornithine in a randomly selected biological sample detected by 2DLC-MS (-). (C) Extracted ion chromatograms of 1-ornithine in a randomly selected biological sample detected by 2DLC-MS (+). (D) Three-dimensional chromatographic peak of taurine detected by GC $\times$ GC-MS. (E) Extracted ion chromatograms of taurine in a randomly selected biological sample detected by 2DLC-MS (-). (F) Extracted ion chromatograms of taurine in a randomly selected biological sample detected by 2DLC-MS (+).

Therefore, the changes of its abundance levels between G1 and G2 detected by 2DLCMS (-) and 2DLC-MS (+) were very similar with fold-change of 1.8 and 1.7, respectively. However, the instrument response of taurine in GC×GC-MS was low and its chromatographic peak in the second dimension GC was very poor (Figure 5.7(D)). Furthermore, the chromatographic peak of taurine overlapped with an abundant peak. For those reasons, the data analysis software ChromaTOF could not accurately quantify the abundance of taurine, and resulted in a large variation in its fold-change compared with the fold-change calculated from the 2DLC-MS (-) and 2DLC-MS (+) data.

Overall, the GC $\times$ GC-MS, 2DLC-MS (-), and 2DLC-MS (+) platforms implemented in this study are robust for metabolite quantification. Manual analysis of the data reveals that the variation in metabolite quantification was mainly induced by the poor instrument response and the limited accuracy of data analysis software.

\subsubsection{Biomarker Discovery and Pathway Analysis}


For metabolic pathway analysis, we first used the metabolites detected by each platform that had significant changes in their abundance levels between groups as the input of MetaboAnalyst software ${ }^{134}$, to recognize the pathways that were affected by the treatments of rats in $\mathrm{G} 1$ and $\mathrm{G} 2$. From the 41 significant metabolites found from the $\mathrm{GC} \times \mathrm{GC}-\mathrm{MS}$ data, the MetaboAnalyst software produced five pathways with $p \leq 0.05$ (Table 5.9).

Table 5.9. Pathways affected by the treatment difference between groups

Significantly impacted pathways $\quad p$-value $\quad$ Match status

\section{Integrated platforms}

Aminoacyl-tRNA biosynthesis

$1.10 \mathrm{E}-03$

$8 / 69$

Arginine and proline metabolism

2.10E-03 6/44

Valine, leucine and isoleucine biosynthesis

$4.10 \mathrm{E}-03$

$3 / 11$

Butanoate metabolism

$4.30 \mathrm{E}-03$

$4 / 22$

Alanine, aspartate and glutamate metabolism

$5.90 \mathrm{E}-03$

$4 / 24$

Biosynthesis of unsaturated fatty acids

9.10E-03

$5 / 42$

d-Glutamine and d-glutamate metabolism

$9.30 \mathrm{E}-03$

$2 / 5$

Taurine and hypotaurine metabolism

$2.40 \mathrm{E}-02$

$2 / 8$

\section{GC $\times$ GC-MS}

Biosynthesis of unsaturated fatty acids

$2.30 \mathrm{E}-03$

$5 / 42$

Aminoacyl-tRNA biosynthesis

$4.20 \mathrm{E}-03$

$6 / 69$

Butanoate metabolism

$1.30 \mathrm{E}-02$

$3 / 22$

Alanine, aspartate and glutamate metabolism

$1.70 \mathrm{E}-02$

$3 / 24$

Valine, leucine and isoleucine biosynthesis

$2.50 \mathrm{E}-02$

$2 / 11$

\section{DLC-MS (-)}

Taurine and hypotaurine metabolism

1.20E-03 $2 / 8$

Valine, leucine and isoleucine biosynthesis

Citrate cycle (TCA cycle)

$2.40 \mathrm{E}-03 \quad 2 / 11$

Butanoate metabolism

$8.00 \mathrm{E}-03$

$2 / 20$

$9.60 \mathrm{E}-03 \quad 2 / 22$

Alanine, aspartate and glutamate metabolism

$1.10 \mathrm{E}-02$

$2 / 24$

D-Glutamine and D-glutamate metabolism

$3.50 \mathrm{E}-02$

$1 / 5$

Primary bile acid biosynthesis

$3.90 \mathrm{E}-02$

$2 / 46$

2DLC-MS (+)

Arginine and proline metabolism

2.30E-04 $\quad 4 / 44$

Taurine and hypotaurine metabolism

$1.50 \mathrm{E}-03$

$2 / 8$

Aminoacyl-tRNA biosynthesis

$1.40 \mathrm{E}-02$

$3 / 69$

Phenylalanine, tyrosine and tryptophan biosynthesis

$3.10 \mathrm{E}-02$

$1 / 4$

Cyanoamino acid metabolism

$4.60 \mathrm{E}-02$

$1 / 6$ 
Likewise, MetaboAnalyst showed that seven pathways were statistically significant using the 13 significant metabolites detected from the 2DLC-MS (-) data, and 5 pathways using the 11 significant metabolites detected from the 2DLC-MS (+) data. We then combined all 58 significant metabolites detected from the three platforms and used them as the input of MetaboAnalyst software. By doing so, eight pathways were considered as significantly impacted pathways (Table 5.9).

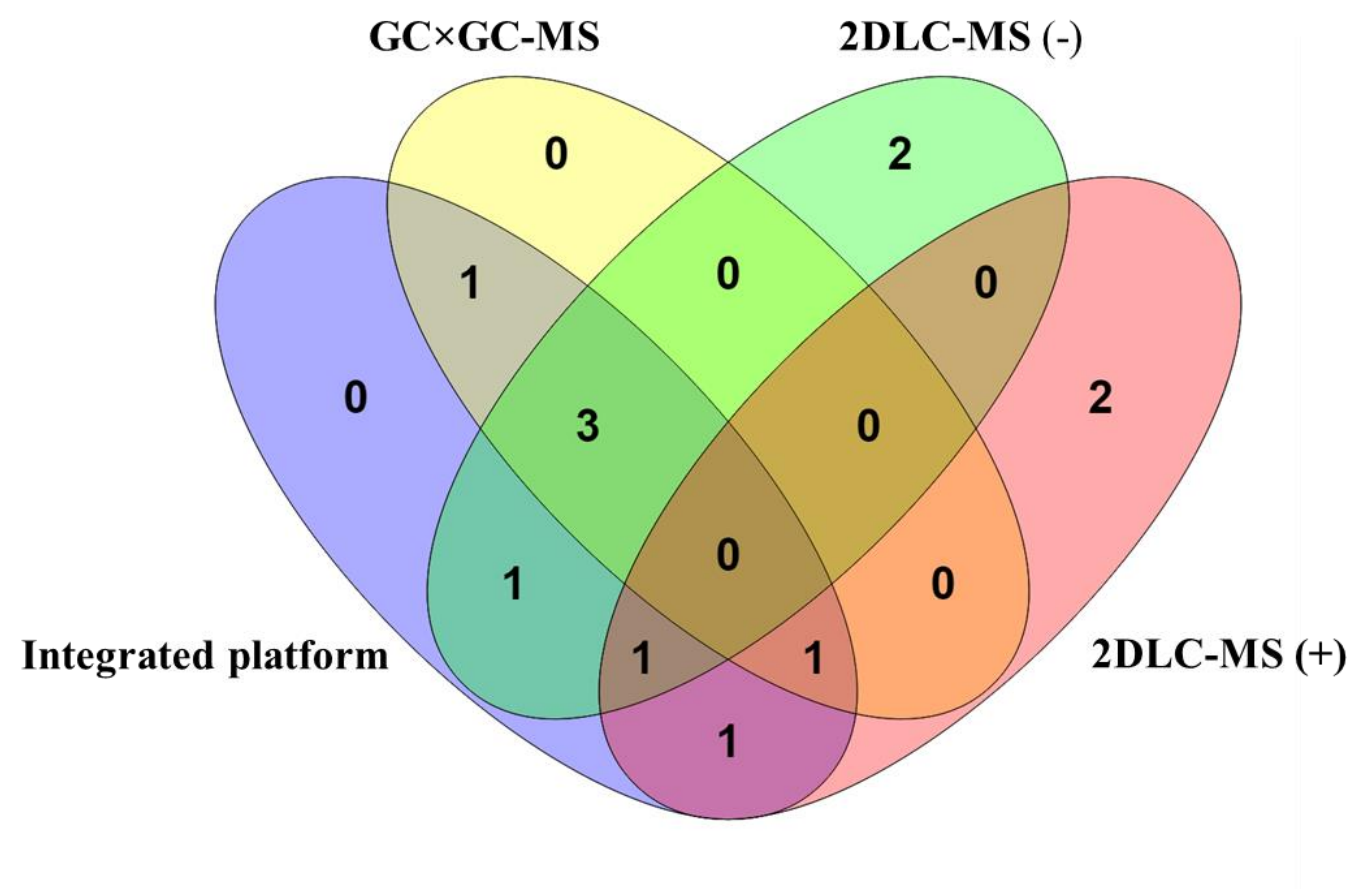

Figure 5.8. Overlap of the pathways that were affected using different sets of significant metabolites for metabolic pathway analysis.

Figure 5.8 shows the overlap of the pathways using different sets of significant metabolites for metabolic pathway analysis. Compared with the results of pathway analysis using the data acquired by individual platform, the confidence of pathway assignment using the integrated data was significantly increased. For instance, 8 metabolites associated with 
aminoacyl-tRNA biosynthesis pathway were detected by integrating the data of all three platforms. However, 0, 3 and 6 metabolites were detected by 2DLC-MS (-), 2DLC-MS (+) and GC×GC-MS, respectively.

Using the integrated data acquired from multiple platforms clearly increase the confidence of metabolic pathway analysis. The confidence of assigning others pathways was also increased, including arginine and proline metabolism; valine, leucine and isoleucine biosynthesis; alanine, aspartate and glutamate metabolism; D-glutamine and Dglutamate metabolism; and butanoate metabolism.

We also performed another analysis by matching all detected metabolites to the metabolic pathway regardless whether those metabolites had significant changes in their abundance levels between groups. For example, biosynthesis of unsaturated fatty acid pathway was a significantly impacted pathway as suggested by the 58 significant metabolites detected from the three platforms. Supplementary Table S5 lists the metabolites in this pathway that were detected by different platforms. Docosahexaenoic acid (DHA), linoleic acid (LA), and gamma-linolenic acid (GLA), were only detected by GC×GC-MS. Prostaglandin G2, leukotriene B4, $\mathrm{LXA}_{4} / \mathrm{LXB}_{4}$ were detected by $2 \mathrm{DLC}-\mathrm{MS}$ $(-)$, and stearidonic acid was only detected by 2DLC-MS (+). Detecting more metabolites in a pathway and knowing whether their abundance levels were affected by the biomedical treatments clearly can narrow down to the specific steps in the pathways that were affected by the treatments.

Table 5.10. Detection of metabolites from the "biosynthesis of unsaturated fatty acid pathway" by different platforms 


\begin{tabular}{|lccc|}
\hline Compound Name & $\mathrm{GC} \times \mathrm{GC}-\mathrm{MS}$ & 2DLC-MS(-) & 2DLC-MS(+) \\
\hline$\alpha$-linolenic acid, ALA & $\mathrm{x}^{\mathrm{a}}$ & $\mathrm{X}$ & $\mathrm{X}$ \\
Stearidonic acid & $\mathrm{x}$ & $\mathrm{X}$ & $\mathrm{y}^{\mathrm{b}}$ \\
Arachidonic acid & $\mathrm{y}$ & $\mathrm{Y}$ & $\mathrm{X}$ \\
Eicosapentaenoic acid, EPA & $\mathrm{y}$ & $\mathrm{Y}$ & $\mathrm{Y}$ \\
Docosapentaenoic acid (DPA) & $\mathrm{x}$ & $\mathrm{Y}$ & $\mathrm{Y}$ \\
Docosahexaenoic acid, DHA & $\mathrm{y}$ & $\mathrm{X}$ & $\mathrm{X}$ \\
Linoleic acid, LA & $\mathrm{y}$ & $\mathrm{X}$ & $\mathrm{X}$ \\
Gamma-linolenic acid, GLA & $\mathrm{y}$ & $\mathrm{X}$ & $\mathrm{X}$ \\
Dihomogamma-linolenic acid, & $\mathrm{y}$ & $\mathrm{X}$ & $\mathrm{Y}$ \\
DGLA & $\mathrm{x}$ & $\mathrm{Y}$ & $\mathrm{X}$ \\
PG2 (prostaglandin G2) & $\mathrm{x}$ & $\mathrm{X}$ & $\mathrm{X}$ \\
TXA2 (thromboxane A2) & $\mathrm{x}$ & $\mathrm{Y}$ & $\mathrm{X}$ \\
leukotriene B4 & $\mathrm{x}$ & $\mathrm{Y}$ & $\mathrm{X}$ \\
LXA4 & $\mathrm{x}$ & $\mathrm{Y}$ & $\mathrm{X}$ \\
LXB4 & & & \\
\hline
\end{tabular}

In summary, one analytical platform is not enough to give high metabolite coverage in a metabolic pathway, and the use of only one platform reduces the confidence of metabolic pathway assignment. Integrating the data acquired from multiple platforms not only provides high metabolite coverage, but also increases the confidence of pathway assignment and the confidence of biomarker discovery. The details of the biomarkers and pathways discovered in this study will be described in a separate report.

\subsection{Conclusions}

Polar metabolites extracted from rat livers were analyzed by GC×GC-MS and parallel 2DLC-MS, respectively. $903 \pm 96$ chromatographic peaks were detected by GC $\times$ GC-MS, and 2,467 \pm 144 and 4,422 \pm 153 features were respectively detected by 2DLC-MS (-) and 2DLC-MS (+). Integrating the experimental data acquired from the three platforms clearly increased the metabolite coverage. A total of 58 metabolites were detected with significant 
changes in their abundance levels between groups. Three of the 58 metabolites were detected in two platforms and two in all three platforms. The agreement of metabolite regulation detected by different platforms demonstrated the robustness and accuracy of the three platforms used in the current study. Manual examination showed that the discrepancy of metabolite regulation measured by different platforms was mainly caused by the poor shape of chromatographic peaks. Pathway analysis demonstrated that integrating the results from multiple platforms increased the confidence of metabolic pathway assignment. While the developed method showed excellent results in terms of metabolites coverage and increased confidence of metabolic pathway assignment, however, several factors still need to be worked on for better identification and quantification. Metabolite identification still remains as a major challenge in untargeted metabolomics. While huge number of isotopic peaks (raw data) were generated from the instrument, most of them remained unidentified. Incompleteness of the database contributed to this problem.

Future endeavor would be the combination of all available databases to maximize the metabolite assignment and develop robust bioinformatics tools for accurate metabolite identification. Furthermore, a comparison of the parallel 2DLC-MS results with the comprehensive 2DLC-MS results would enlighten the overall capability of the multidimensional analytical technique for the untargeted metabolomics. 


\section{CHAPTER 6}

\section{SUMMARY AND CONCLUSIONS}

Metabolomics is a branch of 'omics study that is still being enriched by more instrumental development as well as the method development. While GC×GC-MS has extensively been used in different scientific fields, its use in untargeted metabolomics is limited due to the complicated data analysis especially metabolites identification. This dissertation summarizes our efforts in developing innovative methods to accurately calculate the second dimension retention index from $\mathrm{GC} \times \mathrm{GC}-\mathrm{MS}$ data. We first developed a novel approach using the retention information of entire reference compounds to construct the retention surface for calculation of the second-dimension retention index for a query compound. This approach reduces the variation and increased the accuracy of the

${ }^{2} I$ calculation, which in turns greatly increase the confidence of metabolite identification. In Chapter Three, we developed a method that considered the second-dimension separation as temperature gradient mode and avoided the hold-up time calculation. In Chapter Four, we developed a universal reference method that employs multiple classes of reference compounds as reference compounds to greatly increase the coverage of retention map area, and therefore to eliminate the use of extrapolation. Our long term long is to apply the developed methods to large scale metabolomics to aid metabolite identification in GC $\times$ GCMS. 
A majority of current metabolomics studies are carried out using a single analytical platform such as LC-MS or GC-MS, and therefore have limited metabolite coverage. While GC $\times$ GC-MS and 2DLC-MS has been separately used in metabolomics for high metabolite coverage, each of them still has limited metabolite coverage. In this dissertation, GC $\times$ GC-MS and parallel 2DLC-MS, for the first time, were used to analyze the same biological sample. Each of these analytical platforms separates metabolites using different mechanisms. Therefore, integration of the metabolomics data generated from these platforms can greatly increase the metabolite coverage. Furthermore, some metabolites can be identified and quantified by both analytical platforms. Detecting the same metabolite on multiple platforms provides a chance to validate the accuracy of metabolite quantification. The analysis results of analyzing the metabolite extract of a biological sample using GC $\times$ GC-MS and parallel 2DLC-MS demonstrated that the integrated platform not only provides high metabolite coverage and accurate metabolite quantification, but also reliable metabolic pathway assignment. 


\section{REFERENCES}

1. Wishart, D.S. Advances in metabolite identification. Bioanalysis 3, 1769-1782 (2011).

2. Kind, T., Scholz, M. \& Fiehn, O. How Large Is the Metabolome? A Critical Analysis of Data Exchange Practices in Chemistry. Plos One 4(2009).

3. Sugimoto, M., Wong, D.T., Hirayama, A., Soga, T. \& Tomita, M. Capillary electrophoresis mass spectrometry-based saliva metabolomics identified oral, breast and pancreatic cancer-specific profiles. Metabolomics : Official journal of the Metabolomic Society 6, 7895 (2010).

4. He, L., et al. Simultaneous quantification of straight-chain and branched-chain short chain fatty acids by gas chromatography mass spectrometry. Journal of Chromatography $B$ 1092, 359-367 (2018).

5. Scalbert, A., et al. Mass-spectrometry-based metabolomics: limitations and recommendations for future progress with particular focus on nutrition research. Metabolomics: Official journal of the Metabolomic Society 5, 435-458 (2009).

6. De Souza, A.G., MacCormack, T.J., Wang, N., Li, L. \& Goss, G.G. Large-scale proteome profile of the zebrafish (Danio rerio) gill for physiological and biomarker discovery studies. Zebrafish 6, 229-238 (2009).

7. Dunn, W.B., Bailey, N.J. \& Johnson, H.E. Measuring the metabolome: current analytical technologies. Analyst 130, 606-625 (2005).

8. Wishart, D.S., et al. The human cerebrospinal fluid metabolome. Journal of Chromatography B 871, 164-173 (2008).

9. $\quad$ Psychogios, N., et al. The human serum metabolome. Plos One 6, e16957 (2011).

10. van der Werf, M.J., Overkamp, K.M., Muilwijk, B., Coulier, L. \& Hankemeier, T. Microbial metabolomics: toward a platform with full metabolome coverage. Analytical biochemistry 370, 17-25 (2007).

11. Reo, N.V. NMR-based metabolomics. Drug and chemical toxicology 25, 375-382 (2002).

12. Zhou, B., Xiao, J.F., Tuli, L. \& Ressom, H.W. LC-MS-based metabolomics. Molecular BioSystems 8, 470-481 (2012).

13. Fancy, S.-A. \& Rumpel, K. GC-MS-based metabolomics. Biomarker Methods in Drug Discovery and Development, 317-340 (2008).

14. Garcia, A. \& Barbas, C. Gas chromatography-mass spectrometry (GC-MS)-based metabolomics. Metabolic profiling: Methods and protocols, 191-204 (2011).

15. Stein, S.E. An integrated method for spectrum extraction and compound identification from gas chromatography/mass spectrometry data. J Am Soc Mass Spectr 10, 770-781 (1999).

16. Stein, S.E. \& Scott, D.R. Optimization and testing of mass spectral library search algorithms for compound identification. J Am Soc Mass Spectrom 5, 859-866 (1994). 
17. Atwater, B.L., Stauffer, D.B., Mclafferty, F.W. \& Peterson, D.W. Reliability Ranking and Scaling Improvements to the Probability Based Matching System for Unknown MassSpectra. Analytical Chemistry 57, 899-903 (1985).

18. Tabb, D.L., MacCoss, M.J., Wu, C.C., Anderson, S.D. \& Yates, J.R. Similarity among Tandem Mass Spectra from Proteomic Experiments: Detection, Significance, and Utility. Analytical Chemistry 75, 2470-2477 (2003).

19. Beer, I., Barnea, E., Ziv, T. \& Admon, A. Improving large-scale proteomics by clustering of mass spectrometry data. Proteomics 4, 950-960 (2004).

20. Craig, R., Cortens, J.C., Fenyo, D. \& Beavis, R.C. Using annotated peptide mass spectrum libraries for protein identification. Journal of Proteome Research 5, 1843-1849 (2006).

21. Frewen, B.E., Merrihew, G.E., Wu, C.C., Noble, W.S. \& MacCoss, M.J. Analysis of peptide MS/MS spectra from large-scale proteomics experiments using spectrum libraries. Analytical Chemistry 78, 5678-5684 (2006).

22. Hertz, H.S., Hites, R.A. \& Biemann, K. Identification of Mass Spectra by ComputerSearching a File of Known Spectra. Analytical Chemistry 43, 681-\& (1971).

23. Stein, S.E. \& Scott, D.R. Optimization and testing of mass spectral library search algorithms for compound identification. J Am Soc Mass Spectr 5, 859-866 (1994).

24. Horai, H., Arita, M. \& Nishioka, T. Comparison of ESI-MS spectra in MassBank database. Int Conf Biomed, 853-857 (2008).

25. Koo, I., Kim, S. \& Zhang, X. Comparative analysis of mass spectral matching-based compound identification in gas chromatography-mass spectrometry. Journal of Chromatography A 1298, 132-138 (2013).

26. Kim, S. \& Zhang, X. Discovery of false identification using similarity difference in GC-MSbased metabolomics. J Chemometr 29, 80-86 (2015).

27. Zhang, X., et al. An automated method for the analysis of stable isotope labeling data in proteomics. J Am Soc Mass Spectr 16, 1181-1191 (2005).

28. Vandendool, H. \& Kratz, P.D. A Generalization of the Retention Index System Including Linear Temperature Programmed Gas-Liquid Partition Chromatography. Journal of chromatography 11, 463-471 (1963).

29. Kováts, E. Gas-chromatographische Charakterisierung organischer Verbindungen. Teil 1: Retentionsindices aliphatischer Halogenide, Alkohole, Aldehyde und Ketone. Helvetica Chimica Acta 41, 1915-1932 (1958).

30. Lisec, J., Schauer, N., Kopka, J., Willmitzer, L. \& Fernie, A.R. Gas chromatography mass spectrometry-based metabolite profiling in plants. Nat Protoc 1, 387-396 (2006).

31. Dunn, W.B., et al. Procedures for large-scale metabolic profiling of serum and plasma using gas chromatography and liquid chromatography coupled to mass spectrometry. Nat Protoc 6, 1060-1083 (2011).

32. Zhang, J., et al. iMatch: A retention index tool for analysis of gas chromatography-mass spectrometry data. Journal of Chromatography A 1218, 6522-6530 (2011).

33. Wei, X.L., Koo, I., Kim, S. \& Zhang, X. Compound identification in GC-MS by simultaneously evaluating the mass spectrum and retention index. Analyst 139, 2507-2514 (2014).

34. Stoll, D.R. \& Carr, P.W. Two-Dimensional Liquid Chromatography: A State of the Art Tutorial. Analytical chemistry (2016).

35. Bertsch, W. Two-Dimensional Gas Chromatography. Concepts, Instrumentation, and Applications-Part 1: Fundamentals, Conventional Two-Dimensional Gas Chromatography, Selected Applications. Journal of High Resolution Chromatography 22, 647-665 (1999). 
36. Mondello, L., Tranchida, P.Q., Dugo, P. \& Dugo, G. Comprehensive two-dimensional gas chromatography-mass spectrometry: A review. Mass spectrometry reviews 27, 101-124 (2008).

37. Shellie, R.A. \& Marriott, P.J. Comprehensive two-dimensional gas chromatography-mass spectrometry analysis of Pelargonium graveolens essential oil using rapid scanning quadrupole mass spectrometry. Analyst 128, 879-883 (2003).

38. von Muhlen, C. \& Marriott, P.J. Retention indices in comprehensive two-dimensional gas chromatography. Analytical and bioanalytical chemistry 401, 2351-2360 (2011).

39. Jiang, M., Kulsing, C., Nolvachai, Y. \& Marriott, P.J. Two-dimensional retention indices improve component identification in comprehensive two-dimensional gas chromatography of saffron. Analytical chemistry 87, 5753-5761 (2015).

40. Western, R.J. \& Marriott, P.J. Retention correlation maps in comprehensive twodimensional gas chromatography. Journal of Separation Science 25, 831-838 (2002).

41. Western, R.J. \& Marriott, P.J. Methods for generating second dimension retention index data in comprehensive two-dimensional gas chromatography. Journal of Chromatography A 1019, 3-14 (2003).

42. Koo, I., Shi, X., Kim, S. \& Zhang, X. iMatch2: Compound identification using retention index for analysis of gas chromatography-mass spectrometry data. J Chromatogr A 1337, 202210 (2014).

43. Ressom, H.W., et al. Utilization of metabolomics to identify serum biomarkers for hepatocellular carcinoma in patients with liver cirrhosis. Analytica chimica acta $\mathbf{7 4 3}, \mathbf{9 0 -}$ 100 (2012).

44. Chen, F., Xue, J., Zhou, L., Wu, S. \& Chen, Z. Identification of serum biomarkers of hepatocarcinoma through liquid chromatography/mass spectrometry-based metabonomic method. Analytical and bioanalytical chemistry 401, 1899 (2011).

45. Liu, S.-Y., Zhang, R.-L., Kang, H., Fan, Z.-J. \& Du, Z. Human liver tissue metabolic profiling research on hepatitis B virus-related hepatocellular carcinoma. World journal of gastroenterology: WJG 19, 3423 (2013).

46. Harn, H.J., et al. Exploration of Potential Tumor Markers for Lung Adenocarcinomas by Two-Dimensional Gel Electrophoresis Coupled with Nano-LC/MS/MS. Journal of the Chinese Chemical Society 57, 180-188 (2010).

47. Theodoridis, G., et al. LC-MS based global metabolite profiling of grapes: solvent extraction protocol optimisation. Metabolomics 8, 175-185 (2012).

48. Xu, X., et al. MFG-E8 inhibits Abeta-induced microglial production of cathelicidin-related antimicrobial peptide: A suitable target against Alzheimer's disease. Cellular immunology (2018).

49. Müller, D.C., et al. Metabolomics using GC-TOF-MS followed by subsequent GC-FID and HILIC-MS/MS analysis revealed significantly altered fatty acid and phospholipid species profiles in plasma of smokers. Journal of Chromatography B 966, 117-126 (2014).

50. Cacciola, F., Jandera, P. \& Mondello, L. Comparison of high-temperature gradient heartcutting and comprehensive LC $\times$ LC systems for the separation of phenolic antioxidants. Chromatographia 66, 661-667 (2007).

51. Breidbach, A. \& Ulberth, F. Two-dimensional heart-cut LC-LC improves accuracy of exactmatching double isotope dilution mass spectrometry measurements of aflatoxin B1 in cereal-based baby food, maize, and maize-based feed. Analytical and bioanalytical chemistry 407, 3159-3167 (2015).

52. Stoll, D.R., et al. Fast, comprehensive two-dimensional liquid chromatography. J Chromatogr A 1168, 3-43 (2007). 
53. Erni, F. \& Frei, R. Two-dimensional column liquid chromatographic technique for resolution of complex mixtures. J Chromatogr A 149, 561-569 (1978).

54. Klavins, K., Drexler, H., Hann, S. \& Koellensperger, G. Quantitative Metabolite Profiling Utilizing Parallel Column Analysis for Simultaneous Reversed-Phase and Hydrophilic Interaction Liquid Chromatography Separations Combined with Tandem Mass Spectrometry. Anal. Chem. 86, 4145-4150 (2014).

55. Winnike, J.H., et al. Comparison of GC-MS and GCxGC-MS in the Analysis of Human Serum Samples for Biomarker Discovery. Journal of Proteome Research 14, 1810-1817 (2015).

56. Shi, X., et al. Hepatic and fecal metabolomic analysis of the effects of Lactobacillus rhamnosus GG on alcoholic fatty liver disease in mice. J. Proteome Res. 14, 1174-1182 (2015).

57. Shi, X., et al. Metabolomic analysis of the effects of chronic arsenic exposure in a mouse model of diet-induced fatty liver disease. J. Proteome Res. 13, 547-554 (2014).

58. Shi, X., et al. Metabolomic Analysis of the Effects of Polychlorinated Biphenyls in Nonalcoholic Fatty Liver Disease. Journal of Proteome Research 11, 3805-3815 (2012).

59. Han, J., Datla, R., Chan, S. \& Borchers, C.H. Mass spectrometry-based technologies for high-throughput metabolomics. Bioanalysis 1, 1665-1684 (2009).

60. Winnike, J.H., et al. Comparison of GC-MS and GCX GC-MS in the analysis of human serum samples for biomarker discovery. J Proteome Res 14, 1810-1817 (2015).

61. Shi, X., et al. Hepatic and fecal metabolomic analysis of the effects of Lactobacillus rhamnosus GG on alcoholic fatty liver disease in mice. J Proteome Res 14, 1174-1182 (2015).

62. Shi, X., et al. Metabolomic analysis of the effects of chronic arsenic exposure in a mouse model of diet-induced fatty liver disease. J Proteome Res 13, 547-554 (2013).

63. Shi, X., et al. Metabolomic analysis of the effects of polychlorinated biphenyls in nonalcoholic fatty liver disease. J Proteome Res 11, 3805-3815 (2012).

64. Han, J., Datla, R., Chan, S. \& Borchers, C.H. Mass spectrometry-based technologies for high-throughput metabolomics. (2009).

65. Marriott, P. \& Shellie, R. Principles and applications of comprehensive two-dimensional gas chromatography. TrAC Trends in Analytical Chemistry 21, 573-583 (2002).

66. Tranchida, P.Q., Purcaro, G., Dugo, P., Mondello, L. \& Purcaro, G. Modulators for comprehensive two-dimensional gas chromatography. TrAC Trends in Analytical Chemistry 30, 1437-1461 (2011).

67. Murray, J.A. Qualitative and quantitative approaches in comprehensive two-dimensional gas chromatography. J Chromatogr A 1261, 58-68 (2012).

68. Phillips, J.B. \& Beens, J. Comprehensive two-dimensional gas chromatography: a hyphenated method with strong coupling between the two dimensions. J Chromatogr $A$ 856, 331-347 (1999).

69. Ong, R.C.Y. \& Marriott, P.J. A Review of Basic Concepts in Comprehensive TwoDimensional Gas Chromatography. Journal of Chromatographic Science 40, 276-291 (2002).

70. Adahchour, M., Beens, J., Vreuls, R.J.J. \& Brinkman, U.A.T. Recent developments in comprehensive two-dimensional gas chromatography (GC×GC). TrAC Trends in Analytical Chemistry 25, 821-840 (2006).

71. Adahchour, M., Beens, J. \& Brinkman, U.A.T. Recent developments in the application of comprehensive two-dimensional gas chromatography. J Chromatogr A 1186, 67-108 (2008). 
72. Beens, J., Tijssen, R. \& Blomberg, J. Comprehensive Two-Dimensional Gas Chromatography $(\mathrm{GC} \times \mathrm{GC})$ as a Diagnostic Tool. Journal of High Resolution Chromatography 21, 63-64 (1998).

73. Beens, J., Tijssen, R. \& Blomberg, J. Prediction of comprehensive two-dimensional gas chromatographic separations. J Chromatogr A 822, 233-251 (1998).

74. Bieri, S. \& Marriott, P.J. Dual-Injection System with Multiple Injections for Determining Bidimensional Retention Indexes in Comprehensive Two-Dimensional Gas Chromatography. Analytical Chemistry 80, 760-768 (2008).

75. Bieri, S. \& Marriott, P.J. Generating Multiple Independent Retention Index Data in Dualsecondary Column Comprehensive Two-dimensional Gas Chromatography. Analytical Chemistry 78, 8089-8097 (2006).

76. Zhao, Y., et al. A method of calculating the second dimension retention index in comprehensive two-dimensional gas chromatography time-of-flight mass spectrometry. J Chromatogr A 1218, 2577-2583 (2011).

77. Pang, T., Zhu, S., Lu, X. \& Xu, G. Identification of unknown compounds on the basis of retention index data in comprehensive two-dimensional gas chromatography. Journal of separation science 30, 868-874 (2007).

78. Pacáková, V. \& Feltl, L. Chromatographic retention indices: an aid to identification of organic compounds, (Ellis Horwood Ltd, 1992).

79. Rang, S., Kuningas, K., Orav, A. \& Eisen, O. Capillary gas chromatography of n-alkyness: II. Variation of retention indices with temperature. J Chromatogr A 128, 53-58 (1976).

80. Hala, S., Eyem, J., Burkhard, J. \& Landa, S. Retention Indices of Adamantanes. Journal of Chromatographic Science 8, 203-209 (1970).

81. Döring, C.E., Estel, D., Weber, J., Zimmermann, G. \& Zschummel, D. Kapillargaschromatographische Untersuchungen an homo- und codimeren C5-Dienen. -Einige Zusammenhänge zwischen Retentionsverhalten und Struktur bzw. Konfiguration. Journal für Praktische Chemie 313, 1081-1091 (1971).

82. Shi, X., et al. Metabolomic Analysis of the Effects of Chronic Arsenic Exposure in a Mouse Model of Diet-Induced Fatty Liver Disease. J Proteome Res 13, 547-554 (2014).

83. Linstrom, P. Nist standard reference database number 69. NIST Chemistry WebBook (2003).

84. Kim, S. \& Zhang, X. Discovery of false identification using similarity difference in GC-MSbased metabolomics. J Chemometr 29, 80-86 (2015).

85. Koo, I., Kim, S. \& Zhang, X. Comparative analysis of mass spectral matching-based compound identification in gas chromatography mass spectrometry. J. Chromatogr. A 1298, 132-138 (2013).

86. Koo, I., et al. Elder: a compound identification tool for gas chromatography-mass spectrometry data. J. Chromatogr. A 1448, 107-114 (2016).

87. Prodhan, M.A.I., Yin, X., Kim, S., McClain, C. \& Zhang, X. Surface fitting for calculating the second dimension retention index in comprehensive two-dimensional gas chromatography mass spectrometry. Journal of Chromatography A 1539, $62-70$ (2018).

88. Koo, I., Zhao, Y., Zhang, J., Kim, S. \& Zhang, X. A method of calculating the second dimension hold-up time for comprehensive two-dimensional gas chromatography. $J$ Chromatogr A 1260, 193-199 (2012).

89. Klee, M.S. \& Blumberg, L.M. Measurement of retention in comprehensive twodimensional gas chromatography using flow modulation with methane dopant. J Chromatogr A 1217, 1830-1837 (2010). 
90. Quintanilla-López, J.E., Lebrón-Aguilar, R. \& García-Domínguez, J.A. Hold-up time in gas chromatography: $\mathrm{V}$. Dependence of the retention of $n$-alkanes on the chromatographic variables in isothermal gas chromatography. J Chromatogr A 878, 125-135 (2000).

91. Lebrón-Aguilar, R., García-Domínguez, J.A. \& Quintanilla-López, J.E. Hold-up time in gas chromatography1Presented at the 26th Meeting of the Group of Chromatography and Related Techniques of the Spanish Royal Society of Chemistry, Almería, October, 1997.1: IV. Improved determination of Kováts' retention indices. J Chromatogr A 805, 161-168 (1998).

92. Quintanilla-López, J.E., Lebrón-Aguilar, R. \& García-Domínguez, J. The hold-up time in gas chromatography II. Validation of the estimation based on the concept of a zero carbon atoms alkane. J Chromatogr A 767, 127-136 (1997).

93. Veenaas, C. \& Haglund, P. A retention index system for comprehensive two-dimensional gas chromatography using polyethylene glycols. J Chromatogr A (2017).

94. Zhang, J., et al. iMatch: a retention index tool for analysis of gas chromatography-mass spectrometry data. Journal of chromatography. A 1218, 6522-6530 (2011).

95. Lee, M.L., Vassilaros, D.L. \& White, C.M. Retention indices for programmed-temperature capillary-column gas chromatography of polycyclic aromatic hydrocarbons. Analytical chemistry 51, 768-773 (1979).

96. Warner, D.R., et al. Ethanol and unsaturated dietary fat induce unique patterns of hepatic $\omega-6$ and $\omega-3$ PUFA oxylipins in a mouse model of alcoholic liver disease. Plos One 13, e0204119 (2018).

97. Koo, I., Kim, S. \& Zhang, X. Comparative analysis of mass spectral matching-based compound identification in gas chromatography-mass spectrometry. J Chromatogr A 1298, 132-138 (2013).

98. Wei, X., Koo, I., Kim, S. \& Zhang, X. Compound identification in GC-MS by simultaneously evaluating the mass spectrum and retention index. Analyst 139, 2507-2514 (2014).

99. Castello, G. Retention index systems: alternatives to the n-alkanes as calibration standards. J Chromatogr A 842, 51-64 (1999).

100. Raymer, J., Wiesler, D. \& Novotny, M. Structure-retention studies of model ketones by capillary gas chromatography. J Chromatogr A 325, 13-22 (1985).

101. Kersten, B.R., Poole, C.F. \& Furton, K.G. Ambiguities in the determination of McReynolds stationary phase constants. J Chromatogr A 411, 43-59 (1987).

102. Hawkes, S.J. Letters to the Editor: Reply. Journal of Chromatographic Science 10, 536-536 (1972).

103. Ashes, J. \& Haken, J. Gas chromatography of homologous esters: VI. Strucgure-retention increments of aliphatic esters. J Chromatogr A 101, 103-123 (1974).

104. Novák, J. \& Rúžičková, J. Generalization of the gas chromatographic retention index system. J Chromatogr A 91, 79-88 (1974).

105. Prodhan, M.A.I., Yin, X., Kim, S., McClain, C. \& Zhang, X. Surface fitting for calculating the second dimension retention index in comprehensive two-dimensional gas chromatography mass spectrometry. J Chromatogr $A$.

106. Zhang, J., et al. iMatch: A retention index tool for analysis of gas chromatography-mass spectrometry data. J Chromatogr A 1218, 6522-6530 (2011).

107. Zhao, J., et al. Histidyl Dipeptide Carnosine Imparts Cardiac Protection During Ischemia Reperfusion Injury by Sequestering Lipid Peroxidation Products and Buffering Intracellular pH. Circulation 138, A16059-A16059 (2018).

108. Warner, D., et al. Lipidomic analysis of liver injury caused by a w6-PUFA-enriched diet and ethanol exposure. The FASEB Journal 31, 947.945-947.945 (2017). 
109. Kuehnbaum, N.L. \& Britz-McKibbin, P. New advances in separation science for metabolomics: resolving chemical diversity in a post-genomic era. Chemical reviews 113, 2437-2468 (2013).

110. Stoll, D.R. \& Carr, P.W. Two-Dimensional Liquid Chromatography: A State of the Art Tutorial. Anal. Chem. 89, 519-531 (2017).

111. Almstetter, M.F., Oefner, P.J. \& Dettmer, K. Comprehensive two-dimensional gas chromatography in metabolomics. Analytical and bioanalytical chemistry 402, 1993-2013 (2012).

112. Higgins Keppler, E.A., Jenkins, C.L., Davis, T.J. \& Bean, H.D. Advances in the application of comprehensive two-dimensional gas chromatography in metabolomics. TrAC Trends in Analytical Chemistry 109, 275-286 (2018).

113. Kim, S., et al. Global Plasma Profiling for Colorectal Cancer-Associated Volatile Organic Compounds: a Proof-of-Principle Study. Journal of Chromatographic Science 57, 385-396 (2019).

114. Warner, D.R., et al. Decreased $\omega 6 / \omega 3$ PUFA ratio attenuates ethanol-induced alterations in intestinal homeostasis, microbiota and liver injury. Journal of lipid research, jlr. RA119000200 (2019).

115. Wei, X., et al. Effects of Dietary Different Doses of Copper and High Fructose Feeding on Rat Fecal Metabolome. J Proteome Res 14, 4050-4058 (2015).

116. Shi, X., et al. Metabolomic analysis of the effects of chronic arsenic exposure in a mouse model of diet-induced fatty liver disease. J Proteome Res 13, 547-554 (2013).

117. Lu, W., Bennett, B.D. \& Rabinowitz, J.D. Analytical strategies for LC-MS-based targeted metabolomics. Journal of Chromatography B 871, 236-242 (2008).

118. Prodhan, A., et al. Integrating Comprehensive Two-dimensional Gas Chromatography Mass Spectrometry and Parallel Two-dimensional Liquid Chromatography Mass Spectrometry for Untargeted Metabolomics. Analyst (2019).

119. Wang, S.Y., et al. A novel stop-flow two-dimensional liquid chromatography-mass spectrometry method for lipid analysis. J Chromatogr A 1321, 65-72 (2013).

120. Warner, D.R., et al. Ethanol and unsaturated dietary fat induce unique patterns of hepatic $\omega-6$ and $\omega-3$ PUFA oxylipins in a mouse model of alcoholic liver disease. Plos One 13, e0204119 (2018).

121. Fairchild, J.N., Horvath, K., Gooding, J.R., Campagna, S.R. \& Guiochon, G. Two-dimensional liquid chromatography/mass spectrometry/mass spectrometry separation of watersoluble metabolites. J Chromatogr A 1217, 8161-8166 (2010).

122. Chung, D.-H., et al. Discovery of a Broad-Spectrum Antiviral Compound That Inhibits Pyrimidine Biosynthesis and Establishes a Type 1 Interferon-Independent Antiviral State. Antimicrobial Agents and Chemotherapy 60, 4552-4562 (2016).

123. Wei, X., et al. MetPP: a computational platform for comprehensive two-dimensional gas chromatography time-of-flight mass spectrometry-based metabolomics. Bioinformatics 29, 1786-1792 (2013).

124. Koo, I., Shi, X., Kim, S. \& Zhang, X. iMatch2: Compound identification using retention index for analysis of gas chromatography-mass spectrometry data. J Chromatogr A 1337, 202210 (2014).

125. Wei, X., et al. Data Preprocessing Method for Liquid Chromatography-Mass Spectrometry Based Metabolomics. Anal. Chem. 84, 7963-7971 (2012).

126. Wei, X., et al. Analysis of stable isotope assisted metabolomics data acquired by high resolution mass spectrometry. Anal. Methods 9, 2275-2283 (2017). 
127. Wei, X., et al. Data dependent chromatographic peak model-based spectrum deconvolution for analysis of LC-MS data. Anal. Chem. 86, 2156-2165 (2014).

128. Wei, X., et al. MetSign: A Computational Platform for High-Resolution Mass Spectrometry-Based Metabolomics. Anal. Chem. 83, 7668-7675 (2011).

129. Kim, S., Koo, I., Fang, A.Q. \& Zhang, X. Smith-Waterman peak alignment for comprehensive two-dimensional gas chromatography-mass spectrometry. BMC Bioinformatics 12(2011).

130. Koo, I., Kim, S. \& Zhang, X. Comparative analysis of mass spectral matching-based compound identification in gas chromatography-mass spectrometry. J Chromatogr A 1298, 132-138 (2013).

131. He, L., et al. Simultaneous quantification of straight-chain and branched-chain short chain fatty acids by gas chromatography mass spectrometry. Journal of chromatography. $B$, Analytical technologies in the biomedical and life sciences 1092, 359-367 (2018).

132. Prodhan, M.A.I., Sleman, A.A., Kim, S., McClain, C. \& Zhang, X. Generalization of Reference System for Calculating the Second Dimension Retention Index in GC $\times$ GC-MS. Journal of Analysis and Testing 2, 263-273 (2018).

133. da Silva, R.R., Dorrestein, P.C. \& Quinn, R.A. Illuminating the dark matter in metabolomics. Proceedings of the National Academy of Sciences 112, 12549-12550 (2015).

134. Chong, J., et al. MetaboAnalyst 4.0: towards more transparent and integrative metabolomics analysis. Nucleic Acids Research 46, W486-W494 (2018). 


\section{APPENDIX \\ LIST OF ABBREVIATIONS}

GC-MS: Gas chromatography mass spectrometry

GC×GC-MS: Two-dimensional gas chromatography mass spectrometry

GC×GC-TOF MS: Two-dimensional Gas Chromatography Time-of-flight Mass

Spectrometry

LC-MS: Liquid chromatography mass spectrometry

2DLC-MS: Two-dimensional liquid chromatography mass spectrometry

EI: Electron Impact

CI : Chemical ionization

ESI: Electro-spray ionization

${ }^{t} R$ : Retention time

$I^{T}$ : Linear retention index

I : Kovats retention index

$t_{R(u)}$ : Retention times of the compound $u$ 
$t_{R(n)}$ : The $n$-alkane with $n$ carbon atoms

$t_{R(n+1)}:$ The $n$-alkane with $n+1$ carbon atoms

${ }^{2} t_{R}^{\prime}:$ Adjusted second dimension retention time

KEGG: Kyoto Encyclopedia of Genes and Genomes

$\mathrm{mL}$ : milliliter

MS: Mass Spectrometry

RP-LC: Reverse Phase Liquid Chromatography

RSD: Relative Standard Deviation

SIR: Selected Ion Recording

SRM: Selected Reaction Monitoring 


\section{CURRICULUM VITAE}

Md Aminul Islam Prodhan

Department of Chemistry, University of Louisville, Louisville, KY 40208

Phone: 502-509-2480

Email: miprod01@1ouisville.edu

\section{ACADEMIC RECORDS}

- Ph.D., Chemistry, University of Louisville, Louisville, KY (Fall-2019)

Cumulative GPA: 3.72 /4.0

Supervisor: Prof. Xiang Zhang

- M.S., Chemistry, University of Louisville, Louisville, KY (Spring-2018)

Cumulative GPA: 3.87 /4.0

Supervisor: Prof. Xiang Zhang

- B.S., Applied Chemistry \& Chemical Engineering, University of Dhaka, (2012)

Cumulative GPA: $3.54 / 4.0$

\section{RESEARCH EXPERIENCE}

January-2015 - December 2019, Ph.D., University of Louisville, Louisville, Kentucky

- Methods of calculating the second dimension retention index for GC $\times$ GC-MS based metabolomics

$>$ Developed a surface fitting method for second dimension retention index calculation to increase the confidence in compound identification from GC $\times$ GC-MS

$>$ Developed a method to eliminate the need for calculation of the hold-up time of the second dimension column for retention index calculation

$>$ Developed a method for generalization of the reference system to calculate the second dimension retention index in $\mathrm{GC} \times \mathrm{GC}-\mathrm{MS}$ 
Developed a universal reference system for the second dimension retention index calculation to allow the use of any compounds as reference

- Integration of GCXGC-MS and parallel 2DLC-MS for untargeted metabolomics

$>$ Developed an integrated approach for untargeted metabolomics using GC×GC-MS and parallel 2DLC-MS

$>$ Implemented the developed method for analysis of polar metabolites extracted from rate livers

$>$ Achieved high metabolite coverage and accurate metabolite quantification, and high confidence metabolite biomarker discovery \& metabolic pathway assignment

- Simultaneous quantification of straight-chain and branched-chain short chain fatty acids

$>$ Participated in development of a method for simultaneous quantification of straight-chain and branched-chain SCFAs

$>$ Two columns with different stationary phases were hyphenated together to achieve optimal separation of the SCFAs

$>$ Obtained the best LOD and LOQ for detecting all SCFAs from biological samples

\section{INDUSTRY EXPERIENCE}

- One month training on "Industrial Process Unit Operation \& Process Control Technique" in Training Institute for Chemical Industries (TICI); Polash, Narshindi, Bangladesh.

- Intern at Ghorasal Fertilizer Factory Limited (an enterprise of Bangladesh Chemical Industry Corporation) to complete supervision on Chemical Process reaction, Process Control, Mechanical Equipment.

\section{TEACHING EXPERIENCE}

- CHEM 207, INTRO TO CHEM ANALYSIS I, Summer 2015, 2016, 2017; Fall 2015, 2016; Spring 2016, 2017.

- CHEM 208, INTRO TO CHEM ANALYSIS II, Summer 2015, 2016, 2017; Fall 2015; Spring 2016, 2017.

\section{PEER REVIEWED PUBLICATONS}

- Prodhan, M.A.I.; Song, M.; He, L.; Shi, B.; Fang, Y.; Yin, X.; McClain, C.J.; Zhang, $\mathrm{X}$. Integrating comprehensive two-dimensional gas chromatography mass spectrometry and two-dimensional liquid chromatography mass spectrometry for untargeted metabolomics. Analyst 2019, DOI: 10.1039/C9AN00560A. 
- Dennis R. Warner, Jeffrey B. Warner, Josiah E. Hardesty, Ying L. Song, Taylor N. King, Jing X. Kang, Chih-Yu Chen, Shanfu Xie, Fang Yuan, Md Aminul Islam Prodhan, Xipeng Ma, Xiang Zhang, Eric C. Rouchka, Joan Whitlock, Eric C. Li, Gary P. Wang, Craig J. McClain, and Irina A. Kirpich. Beneficial effects of decreased omega-6:omega-3 PUFA ratio on intestinal homeostasis and microbiota are linked to the improvement of liver injury associated with ethanol and LPS administration in mice. Manuscript in preparation.

- Prodhan, M.A.I.; McClain, C.J.; Zhang, X. Comprehensive two-dimensional gas chromatography mass spectrometry-based metabolomics. Cancer metabolomics: technologies and applications, 2019, in press [Invited Book Chapter].

- Prodhan, M.A.I.; Yin, X.; Kim, S.; McClain, C.J.; Zhang, X. Surface fitting for calculating the second dimension retention index in comprehensive twodimensional gas chromatography mass spectrometry. J. Chromatogr. A. 2018, $1539,62-70$.

- He, L.; Prodhan, M.A.I.; Yuan, F.; Yin, X.; Lorkiewicz, P.K.; Wei, X.; Feng, W.; McClain, C.; Zhang, X. Simultaneous quantification of straight-chain and branched-chain short fatty acids by gas chromatography mass spectrometry. $J$. Chromatogr. B. 2018, 1092, 359-367.

- Prodhan, M.A.I.; Sleman, A.A.; Kim, S.; McClain, C.J.; Zhang, X. Generalization of reference system for calculating the second dimension retention index in GC×GC-MS. J. Anal. Test. 2018, 2, 263-273.

- Warner, D.R.; Liu, H.; Dastidar, S.G.; Warner, J.B.; Prodhan, M.A.I.; Yin, X.; Zhang, X.; Feldstein, A.E.; Gao, B.; Prough, R.A.; McClain, C.J.; Kirpich, I.A. Characterization of hepatic $\omega-6$ and $\omega-3$ PUFA oxylipins in a mouse model of ethanol-induced liver injury. PLOS ONE 2018, 13(9): e0204119.

- Kim, S; Yin, X; Prodhan, M.A.I.; Zhang, X.; Zhong, Z; Kato, I. Global Plasma Profiling for Colorectal Cancer-Associated Volatile Organic Compounds: a Proofof-Principle Study. J. Chromatogric science. 2019,57,385-396.

- Chung, D.; Golden, J.E.; Adcock, R.S.; Chu, Y.; Sotsky, J.B.; Cramer, D.E.; Chilton, P.M.; Song, C.; Anantpadma, M.; Schroeder, C.E.; Prodhan, M.A.I.; Yin, $\mathrm{X}$.; Zhang, X. Discovery of a broad-spectrum antiviral compound that inhibits pyrimidine biosynthesis and establishes a type 1 interferon-independent antiviral state. Antimicrob. Agents Chemother. 2016, 60(8), 4552-4562.

\section{PRESENTATIONS}

- Prodhan, M.A.I.; Shi, B.; Yin, X.; Feng, W.; McClain, G.; Zhang, X. Integrating comprehensive two-dimensional gas chromatography mass spectrometry and two- 
dimensional liquid chromatography mass spectrometry for metabolomics. Pittcon 2019, Philadelphia, PA, March 17-21, 2019. (Oral)

- Prodhan, M.A.I.; Shi, B.; Yin, X.; Feng, W.; McClain, G.; Zhang, X. Integrated parallel two-dimensional liquid chromatography mass spectrometry and comprehensive two-dimensional gas chromatography mass spectrometry for metabolomics. HPLC 2018, Washington, DC, July 29-August 2, 2018. (Oral)

- Prodhan, M.A.I.; Yin, X.; Kim, S.; McClain, C.J.; Zhang, X. Calculation of the Second Dimension Retention Index in GCXGC-MS Excluding Hold-up Time Calculation. $70^{\text {th }}$ Midwest University Analytical Chemistry Conference (MUACC), Athens, OH, October 20-21, 2017. (Oral)

- He, L.; Li, F.; Yuan, F.; Yin, X.; Prodhan, M.A.I.; McClain, G.; Feng, W.; Zhang, $\mathrm{X}$. Relative quantification of metabolites in fecal samples using three analytical platforms and the role of cathelicidin-related antimicrobial peptide in alcoholic liver disease. Pittcon 2019, Philadelphia, PA, March 17-21, 2019. (Oral)

- Prodhan, M.A.I.; Sleman, A.A.; Yin, X.; Lorkiewicz, P.; Kim, S.; McClain, C.J.; Zhang, X. A method of calculating retention index of the second dimension separation in comprehensive two-dimensional gas chromatography mass spectrometry. ASMS 2019, Atlanta, GA, June 2-6, 2019. (Poster)

- Prodhan, M.A.I.; Yin, X.; Kim, S.; McClain, C.J.; Zhang, X. Universal Reference System for Calculating the Second Dimension Retention Index in GCxGC-MS. Research! Louisville- 2018, Louisville, KY, October 8, 2018. (Poster)

- He, L.; Li, F.; Yuan, F.; Yin, X.; Prodhan, M.A.I.; McClain, G.; Feng, W.; Zhang, $X$. Relative quantification of metabolites in fecal samples using three analytical platforms and the role of cathelicidin-related antimicrobial peptide in alcoholic liver disease. Research! Louisville, University of Louisville, Louisville, KY, October 12, 2018. (Poster)

- Prodhan, M.A.I.; Yin, X.; Kim, S.; McClain, C.J.; Zhang, X. Surface Fitting for Calculating the Second Dimension Retention Index in GCxGC-MS. 70 th Midwest University Analytical Chemistry Conference (MUACC), Athens, OH, October 20- 21, 2017. (Poster)

\section{AWARDS AND RECOGNITIONS}

- Institute for Molecular Diversity and Drug Design (IMD3) - Travel Award, University of Louisville, 2017

- Graduate Network in A\&S (GNAS) Travel Award, University of Louisville, 2017

- Department of chemistry Travel Award, University of Louisville, 2019 
- People's Republic of Bangladesh Government Scholarship for Outstanding result in Secondary School Certificate examination.

\section{PROFESSIONAL MEMBERSHIPS}

- American Society for Mass Spectrometry ( ASMS)

- Chinese American chromatography association ( CACA)

\section{SKILLS}

- Analytical instrumentation : HPLC, 2DLC-MS/MS (ThermoScientific QExactive- HF), GC-MS, GC×GC-MS, FT-MS, QqQ-MS, AAS

- Languages

: Fluent in Bengali and English, Elementary level of Arabic and Hindi

- Extra-curricular activities : Ex-scout 